\title{
Silacycle-Templated Intramolecular Diels-Alder Cyclizations for the Diastereoselective Construction of Complex Carbon Skeletons
}

Paul R. Carlson, Alexander S. Burns, Emily A. Shimizu, Shilin Wang, and Scott D. Rychnovsky*

Department of Chemistry, 1102 Natural Sciences II, University of California at Irvine, Irvine, California 92697, United States

\section{Supporting Information}

Table of Contents Page

General Experimental

S2

Experimentals and Compound Characterization

S5

Unsuccessful substrates

S37

Stereochemical assignment of $\mathbf{1 7} \mathbf{a}$ and $\mathbf{1 7 b}$

S39

References

S41

NMR Spectra

X-Ray crystal data for $\mathbf{2 1}$

S122

Calculations

S126 


\section{General Experimental}

All chemicals were purchased from Sigma-Aldrich, Alfa Aesar, TCI, or Fisher Scientific and used without further purification. Deuterated NMR solvents were purchased from Cambridge Isotope Laboratories. Solvents were purchased as ACS grade or better and passed through activated alumina columns prior to use. Unless otherwise stated, reactions were performed in flame dried glassware under an atmosphere of argon. For reactions performed at elevated temperatures, the desired temperature was maintained using a sand bath. Reaction progress was monitored by thin layer chromatography (TLC) using glass plates coated with a $250 \mu \mathrm{m}$ layer of $60 \AA$ silica gel $\left(\mathrm{SiO}_{2}\right)$. TLC plates were visualized using either a UV lamp at $254 \mathrm{~nm}$, potassium permanganate or cerium molybdate (Hanessian's stain). Column chromatography was performed using forced flow on silica gel columns or with an automated purification system on prepacked silica gel columns.

${ }^{1} \mathrm{H}$ NMR spectra were recorded at $500 \mathrm{MHz}$ or $600 \mathrm{MHz}$ using either a Bruker DRX500 (cryoprobe) or Bruker AVANCE600 (cryoprobe) at $298.0 \mathrm{~K} \cdot{ }^{13} \mathrm{C}$ NMR spectra were recorded at $126 \mathrm{MHz}$ or $151 \mathrm{MHz}$ on a Bruker DRX500 (cryoprobe) or Bruker AVANCE600 (cryoprobe) at 298.0 K. Chemical shifts $(\delta)$ are reported in parts per million (ppm) and referenced to the residual solvent peak or to a tetramethylsilane (TMS) standard. NMR data are reported as follows: chemical shift, multiplicity $(\mathrm{s}=$ singlet, $\mathrm{d}=$ doublet, $\mathrm{t}=$ triplet, $\mathrm{q}=$ quartet, $\mathrm{p}=$ pentet, $\mathrm{m}=$ multiplet, $\mathrm{dd}=$ doublet of doublet, $\mathrm{ddd}=$ doublet of doublets of doublets, $\mathrm{dt}=$ doublet of triplets, $\mathrm{dtd}=$ doublet of triplets of doublets, bs $=$ broad singlet), coupling constants $(J)$ in hertz $(\mathrm{Hz})$, and integration. For partially deuterated compounds, the percentage deuterium incorporation is calculated based on the proton integration value at the deuterated position. In ${ }^{13} \mathrm{C}$ NMR spectra of partially deuterated compounds, carbons within 2-4 sigma bonds of the deuterium atom occasionally exhibit a measurably different chemical shift from their fully protiated counterparts, leaving these spectra 
with more carbon signals than expected. High-resolution mass spectrometry was performed using ESI.

\section{General Procedure A: Formation of aldol adducts}

To a round bottom flask equipped with a magnetic stir bar was added a solution of freshly distilled diisopropylamine in dry THF (1.2 equiv, $1.4 \underline{\mathrm{M}})$. The solution was cooled to $-78^{\circ} \mathrm{C}$ and $n$-butyllithium (2.5 $\mathrm{M}$ in hexanes, 1.1 equiv) was added dropwise. The solution was warmed to 0 ${ }^{\circ} \mathrm{C}$ for 5 minutes, after which it was cooled to $-78^{\circ} \mathrm{C}$ and a solution of enone in THF (1.0 equiv, 1.2 $\underline{\mathrm{M}}$ ) was added dropwise. The reaction was stirred at $-78^{\circ} \mathrm{C}$ for 1 hour, at which time a solution of aldehyde or ketone in THF (1.2 equiv, $1.4 \underline{\mathrm{M}}$ ) was added quickly. The reaction was stirred for another 5 minutes before being quenched with a saturated solution of aqueous ammonium chloride ( 0.5 reaction volumes) and warmed to room temperature. The reaction mixture was transferred to a separatory funnel, the organic phase collected, and the aqueous phase extracted with $\mathrm{CH}_{2} \mathrm{Cl}_{2}(3$ $x 1$ reaction volume). The combined organic layers were washed once with brine, dried over sodium sulfate, and concentrated in vacuo. Product mixtures were then purified by flash column chromatography.

\section{General Procedure B: Silacycle formation and Diels-Alder cyclization}

Unless otherwise indicated, aldol adducts are taken as a mixture of syn and anti diastereomers in the same diastereomeric ratio as they were isolated from the previous aldol reaction. Hydroxyketone (1.0 equiv) was dissolved in $\mathrm{CH}_{2} \mathrm{Cl}_{2}(0.20 \underline{\mathrm{M}})$ in a scintillation vial equipped with a septum. This solution was cooled to $0{ }^{\circ} \mathrm{C}$ and a solution of diisopropyldichlorosilane in $\mathrm{CH}_{2} \mathrm{Cl}_{2}(2.0$ equiv, $0.40 \underline{\mathrm{M}})$ was added slowly. Immediately afterwards, a solution of diazabicycloundecene (DBU) in $\mathrm{CH}_{2} \mathrm{Cl}_{2}(6.0$ equiv, $0.40 \underline{\mathrm{M}})$ was added 
slowly. The reaction was stirred at $0{ }^{\circ} \mathrm{C}$ for 10 minutes and then at room temperature for 10 minutes. The reaction vessel was opened to air momentarily and the septum was exchanged with a Teflon-lined cap. The sealed vessel was stirred at $40{ }^{\circ} \mathrm{C}$ for $13 \mathrm{~h}$. Subsequently, the reaction was cooled to room temperature, at which point it was diluted with pyridine ( 0.10 reaction volumes). A $70 \%$ solution of HF in pyridine ( 0.010 reaction volumes) was added slowly and the reaction stirred for 30 minutes, after which a saturated aqueous solution of sodium bicarbonate was added slowly until bubbling had ceased. The resulting biphasic solution was transferred to a separatory funnel and the organic layer collected. The aqueous layer was extracted with $\mathrm{CH}_{2} \mathrm{Cl}_{2}(3 x 1$ reaction volume) and the combined organic layers were washed with a saturated solution of cupric sulfate (2 $x 1$ reaction volume). The combined cupric sulfate layers were back-extracted with one reaction volume of $\mathrm{CH}_{2} \mathrm{Cl}_{2}$, after which the combined organic layers were dried over sodium sulfate and concentrated in vacuo. All Diels-Alder adducts were obtained as a single diastereomer as determined by ${ }^{13} \mathrm{C}$ NMR of the crude reaction mixture. The product mixtures were purified by flash column chromatography.

\section{General Procedure C: DMP oxidation of primary alcohols}

No precaution was taken to exclude air or water from the reaction. To a round bottom flask was added $\mathrm{CH}_{2} \mathrm{Cl}_{2}(0.1 \underline{\mathrm{M}})$, Dess-Martin periodinane (1.5 equiv), primary alcohol (1.0 equiv), and sodium bicarbonate (10 equiv). The reaction was stirred for one hour open to air, after which time it was transferred to a separatory funnel and diluted with water until all sodium bicarbonate was solvated. One reaction volume of a saturated aqueous solution of sodium thiosulfate was added and the organic layer was collected. The aqueous layer was extracted with $\mathrm{CH}_{2} \mathrm{Cl}_{2}(3 \times 1$ reaction volume). The combined organic layers were washed with brine, dried over sodium sulfate, and concentrated in vacuo. The product mixtures were purified by flash column chromatography. 


\section{Experimentals and Compound Characterization}

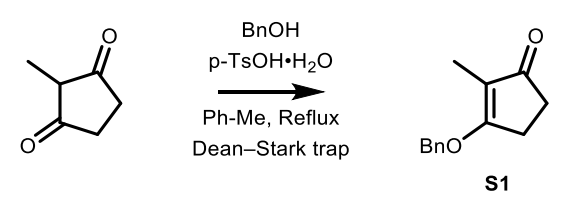

\section{3-(benzyloxy)-2-methylcyclopent-2-en-1-one (S1):}

To a round bottom flask equipped with a stir bar and charged with toluene $(50 \mathrm{~mL})$ was added 2-methylcyclopentane-1,3-dione (2.8 g, $25 \mathrm{mmol}, 1.0$ equiv), tosic acid monohydrate (0.48 g, $2.5 \mathrm{mmol}, 0.10$ equiv), and benzyl alcohol ( $7.8 \mathrm{~mL}, 75 \mathrm{mmol}, 3.0$ equiv). The flask was equipped with a Dean-Stark trap and the reaction mixture was refluxed for 2 hours. Once the starting material had been fully consumed as indicated by TLC analysis, the reaction mixture was allowed to cool to ambient temperature and diluted with a saturated aqueous solution of sodium bicarbonate $(25 \mathrm{~mL})$. The organic layer was collected, and the aqueous layer was extracted with EtOAc $(3 \times 20 \mathrm{~mL})$. The combined organic extracts were washed with brine $(20$ $\mathrm{mL}$ ), dried over sodium sulfate, and concentrated in vacuo. The product mixture was purified by flash column chromatography using $0-70 \%$ EtOAc in hexanes as the eluting solvent to afford $\mathbf{S 1}$ as a yellow oil (4.2 g, 84\% yield): $\mathbf{R}_{f}=0.40$ (3:2, EtOAc:Hex); ${ }^{1} \mathbf{H} \mathbf{N M R}\left(500 \mathrm{MHz}, \mathrm{CDCl}_{3}\right) \delta 7.42-$ $7.28(\mathrm{~m}, 5 \mathrm{H}), 5.21(\mathrm{~s}, 2 \mathrm{H}), 2.64(\mathrm{~s}, 2 \mathrm{H}), 2.44-2.36(\mathrm{~m}, 2 \mathrm{H}), 1.66(\mathrm{~s}, 3 \mathrm{H}) .{ }^{13} \mathbf{C}\left\{{ }^{1} \mathbf{H}\right\} \mathbf{N M R}(126 \mathrm{MHz}$, $\left.\mathrm{CDCl}_{3}\right) \delta 205.4,183.7,136.1,129.0,128.6,127.1,117.1,70.9,33.7,25.4,6.3 ;$ HRMS (ESI) m/z: [M + $\mathrm{Na}]^{+}$Calcd for $\mathrm{C}_{13} \mathrm{H}_{14} \mathrm{O}_{2} \mathrm{Na} 225.0887$; Found 225.0889.

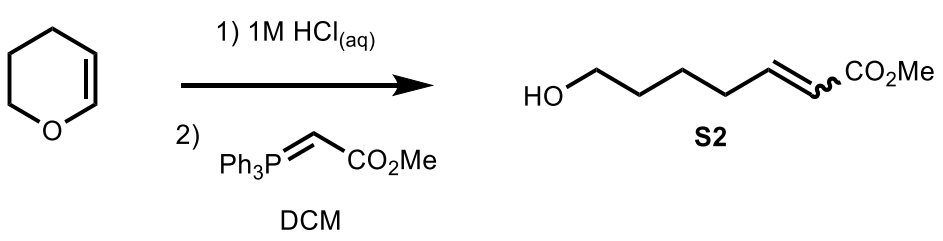

\section{Methyl-7-hydroxyhept-2-enoate (S2):}


No precaution was taken to exclude air or water from the reaction. A round bottom flask charged with dihydropyran (6.4 mL, $75 \mathrm{mmol}, 1.0$ equiv) was cooled to $0{ }^{\circ} \mathrm{C}$ and an aqueous solution of $\mathrm{HCl}(15 \mathrm{~mL}, 0.20 \underline{\mathrm{M}})$ was added slowly. This mixture was stirred at $0{ }^{\circ} \mathrm{C}$ for 15 minutes and then 1 hour at room temperature. The crude reaction mixture was extracted with $\mathrm{CH}_{2} \mathrm{Cl}_{2}(3 \times 10 \mathrm{~mL})$. The combined organic layers were washed with brine $(10.0 \mathrm{~mL})$, dried over sodium sulfate, and concentrated in vacuo. The product mixture was then dissolved in THF $(200 \mathrm{~mL})$ and transferred to a round bottom flask equipped with a magnetic stir bar and a reflux condenser. Methyl-2(triphenyl- $\lambda^{5}$-phosphaneylidene) acetate ( $30 \mathrm{~g}, 90 \mathrm{mmol}, 1.2$ equiv) was added and the solution was refluxed for 13 hours. After this time, the crude reaction mixture was concentrated in vacuo and diluted with a 7:3 $\mathrm{Et}_{2} \mathrm{O}$ : pentanes solution. This mixture was stirred for 45 minutes, during which time triphenylphosphine oxide precipitated as a white solid. The mixture was filtered to remove the phosphine oxide and the filtrate was purified by flash column chromatography using $70 \%$ diethyl ether in pentanes as the eluting solvent to afford pale yellow oil $\mathbf{S 2}$ as an inseparable mixture of olefin isomers $(6.5 \mathrm{~g}, 54 \%$ yield of S2, 10:1 E:Z); all spectral data are consistent with those reported; ${ }^{1}{ }^{1} \mathbf{H}$ NMR (500 MHz, $\left.\mathrm{CDCl}_{3}\right) \delta 6.95(\mathrm{dt}, J=15.6,6.9,1 \mathrm{H}, \mathrm{E}$ isomer), $6.24(\mathrm{dt}, J$ $=11.5,7.6,1 \mathrm{H}, \mathrm{Z}$ isomer), $5.82(\mathrm{dt}, J=15.6,1.5,1 \mathrm{H}, \mathrm{E}$ isomer), $5.78(\mathrm{dt}, J=11.5,1.6,1 \mathrm{H}, \mathrm{Z}$ isomer), 3.71 (s, $3 \mathrm{H}, \mathrm{E}$ isomer), 3.69 (s, 3H, $\mathrm{Z}$ isomer), 3.64 (t, $J=6.2,2 \mathrm{H}, \mathrm{E}$ and $\mathrm{Z}$ isomers), 2.66 (qd, $J=7.6,1.6,1 \mathrm{H}, \mathrm{Z}$ isomer), 2.23 (qd, $J=7.2,1.4,2 \mathrm{H}, \mathrm{E}$ isomer), 1.68 (bs, $1 \mathrm{H}, \mathrm{E}$ and $\mathrm{Z}$ isomers), $1.62-1.48$ ( $\mathrm{m}, 4 \mathrm{H}, \mathrm{E}$ and $\mathrm{Z}$ isomers).
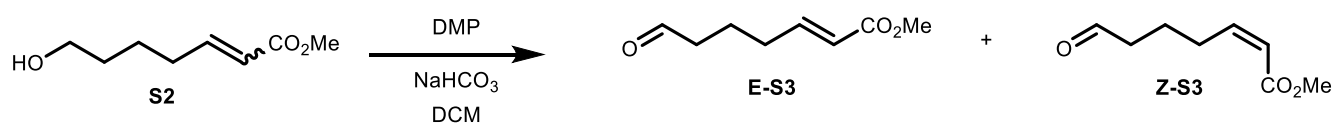

\section{Methyl-7-oxohept-2-enoate (S3):}


Following general procedure C, S2 (6.5 g, 41 mmol, 1.0 equiv) was reacted with Dess-Martin periodinane ( $26 \mathrm{~g}, 0.61 \mathrm{mmol}, 1.5$ equiv) and sodium bicarbonate ( $34 \mathrm{~g}, 0.41 \mathrm{~mol}, 20$ equiv) in $\mathrm{CH}_{2} \mathrm{Cl}_{2}(0.40 \mathrm{~L})$. The product mixture was purified by flash column chromatography using $0-$ 20\% EtOAc in hexanes as the eluting solvent to afford E-S3 $(5.0 \mathrm{~g})$ and $\mathbf{Z}-\mathbf{S 3}(0.31 \mathrm{~g})$ as pale yellow oils ( $83 \%$ combined yield of $\mathbf{S 3})$.

\section{E-S3}

All spectral data are consistent with those reported; ${ }^{1}{ }^{1} \mathbf{H}$ NMR $\left(500 \mathrm{MHz}, \mathrm{CDCl}_{3}\right) \delta 9.73(\mathrm{~s}, 1 \mathrm{H})$, $6.88(\mathrm{dt}, J=16.0,7.0,1 \mathrm{H}), 5.81(\mathrm{~d}, J=15.6,1 \mathrm{H}), 3.69(\mathrm{~s}, 3 \mathrm{H}), 2.45(\mathrm{t}, J=7.1,2 \mathrm{H}), 2.22(\mathrm{q}, J=$ $7.1,2 \mathrm{H}), 1.77(\mathrm{p}, J=7.0,2 \mathrm{H})$.

\section{Z-S3}

All spectral data are consistent with those reported; ${ }^{2}{ }^{1} \mathbf{H}$ NMR $\left(500 \mathrm{MHz}, \mathrm{CDCl}_{3}\right) \delta 9.77(\mathrm{~s}, 1 \mathrm{H})$, $6.18(\mathrm{dt}, J=11.4,7.6 \mathrm{~Hz}, 1 \mathrm{H}), 5.81(\mathrm{~d}, J=11.5 \mathrm{~Hz}, 1 \mathrm{H}), 3.69(\mathrm{~s}, 3 \mathrm{H}), 2.68(\mathrm{q}, J=7.5 \mathrm{~Hz}, 2 \mathrm{H})$, $2.47(\mathrm{t}, J=7.3 \mathrm{~Hz}, 2 \mathrm{H}), 1.83-1.72(\mathrm{~m}, 2 \mathrm{H})$.

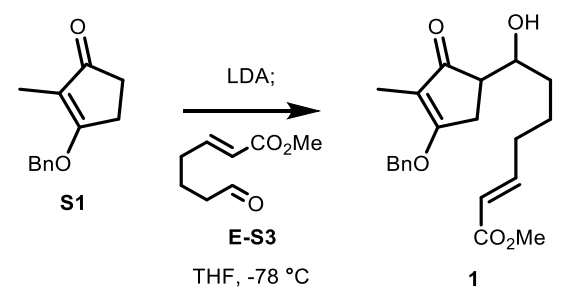

Methyl (E)-7-(4-(benzyloxy)-3-methyl-2-oxocyclopent-3-en-1-yl)-7-hydroxyhept-2-enoate (1):

Following general procedure A, S1 (0.89 g, $4.4 \mathrm{mmol}, 1.0$ equiv) was deprotonated with LDA (0.51 g, $4.8 \mathrm{mmol}, 1.1$ equiv) and reacted with E-S3 (0.75g, $5.3 \mathrm{mmol}, 1.2$ equiv) in THF (10 
$\mathrm{mL})$ at $-78^{\circ} \mathrm{C}$. The product mixture was purified by flash column chromatography using $0-$ $50 \%$ EtOAc in hexanes as the eluting solvent to afford the 1 major and $\mathbf{1}$ minor ( $490 \mathrm{mg}, 30 \%$ combined yield, 1.2:1 dr):

Major diastereomer:

Yellow oil; $\mathbf{R}_{f}=0.39\left(3: 2\right.$ EtOAc:Hex, UV); ${ }^{1} \mathbf{H}$ NMR $\left(600 \mathrm{MHz}, \mathrm{CDCl}_{3}\right) \delta 7.45-7.32(\mathrm{~m}, 5 \mathrm{H})$, $6.96(\mathrm{dt}, J=15.6,6.6 \mathrm{~Hz}, 1 \mathrm{H}), 5.83(\mathrm{~d}, J=15.7 \mathrm{~Hz}, 1 \mathrm{H}), 5.25(\mathrm{~s}, 2 \mathrm{H}), 4.80(\mathrm{~s}, 1 \mathrm{H}), 3.72(\mathrm{~s}, 3 \mathrm{H})$, $3.61(\mathrm{dd}, J=12.9,4.5 \mathrm{~Hz}, 1 \mathrm{H}), 2.79(\mathrm{dd}, J=17.3,6.9 \mathrm{~Hz}, 1 \mathrm{H}), 2.47-2.41(\mathrm{~m}, 1 \mathrm{H}), 2.29(\mathrm{~d}, J=$ $17.3 \mathrm{~Hz}, 1 \mathrm{H}), 2.26-2.18(\mathrm{~m}, 2 \mathrm{H}), 1.78-1.69(\mathrm{~m}, 1 \mathrm{H}), 1.67(\mathrm{~s}, 3 \mathrm{H}), 1.61-1.53(\mathrm{~m}, 1 \mathrm{H}), 1.51-$ $1.42(\mathrm{~m}, 2 \mathrm{H}) ;{ }^{13} \mathbf{C}\left\{{ }^{1} \mathbf{H}\right\} \mathbf{N M R}\left(151 \mathrm{MHz}, \mathrm{CDCl}_{3}\right) \delta$ 208.4, 183.7, 167.2, 149.4, 135.6, 129.0, 128.8, 127.2, 121.3, 116.3, 72.4, 71.4, 51.5, 48.9, 35.1, 32.2, 29.1, 23.5, 6.1; HRMS (ESI) m/z: $[\mathrm{M}+\mathrm{Na}]^{+}$Calcd for $\mathrm{C}_{21} \mathrm{H}_{26} \mathrm{O}_{5} \mathrm{Na}$ 381.1673; Found 381.1672.

Minor Diastereomer:

Yellow oil; $\mathbf{R}_{\boldsymbol{f}}=0.40$ (3:2 EtOAc:Hex, UV); ${ }^{1} \mathbf{H}$ NMR $\left(600 \mathrm{MHz}, \mathrm{CDCl}_{3}\right) \delta 7.40(\mathrm{dd}, J=10.3$, $4.3 \mathrm{~Hz}, 2 \mathrm{H}), 7.38-7.34(\mathrm{~m}, 3 \mathrm{H}), 6.95(\mathrm{dt}, J=15.4,6.9 \mathrm{~Hz}, 1 \mathrm{H}), 5.82(\mathrm{~d}, J=15.7 \mathrm{~Hz}, 1 \mathrm{H}), 5.28$ $-5.23(\mathrm{~m}, 2 \mathrm{H}), 4.13(\mathrm{~s}, 1 \mathrm{H}), 3.72(\mathrm{~s}, 3 \mathrm{H}), 2.71-2.61(\mathrm{~m}, 2 \mathrm{H}), 2.60-2.55(\mathrm{~m}, 1 \mathrm{H}), 2.42(\mathrm{bs}$, 1H), $2.27-2.19(\mathrm{~m}, 2 \mathrm{H}), 1.73-1.68(\mathrm{~m}, 1 \mathrm{H}), 1.65(\mathrm{~s}, 3 \mathrm{H}), 1.53-1.45(\mathrm{~m}, 2 \mathrm{H}), 1.42-1.36(\mathrm{~m}$, $1 \mathrm{H}) ;{ }^{13} \mathbf{C}\left\{{ }^{1} \mathbf{H}\right\}$ NMR $\left(151 \mathrm{MHz}, \mathrm{CDCl}_{3}\right) \delta$ 206.6, 184.6, 167.2, 149.2, 135.9, 129.0, 128.7, 127.2, 121.4, 117.2, 71.2, 69.8, 51.6, 50.6, 34.0, 32.1, 26.6, 24.7, 6.2; HRMS (ESI) m/z: $[\mathrm{M}+\mathrm{Na}]^{+}$ Calcd for $\mathrm{C}_{21} \mathrm{H}_{26} \mathrm{O}_{5} \mathrm{Na} 381.1673$; Found 381.1666. 


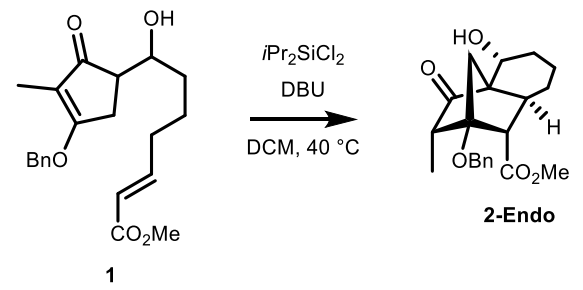

Methyl-2-(benzyloxy)-5-hydroxy-3-methyl-4-oxooctahydro-2H-2,4a-methanonaphthalene1-carboxylate (2-Endo):

Following general procedure B, $1(0.20 \mathrm{~g}, 0.56 \mathrm{mmol}, 1.0$ equiv $)$ was reacted with diisopropyldichlorosilane $(0.20 \mathrm{~mL}, 1.1 \mathrm{mmol}, 2.0$ equiv) and DBU ( $0.50 \mathrm{~mL}, 3.4 \mathrm{mmol}, 6.0$ equiv) in $\mathrm{CH}_{2} \mathrm{Cl}_{2}(6.0 \mathrm{~mL})$ from 0 to $40{ }^{\circ} \mathrm{C}$. The product mixture was purified by flash column chromatography using $0-30 \%$ EtOAc in hexanes as the eluting solvent to afford 2-Endo as a clear oil (0.14 g, 70\% yield, single diastereomer): $\mathbf{R}_{\boldsymbol{f}}=0.39$ (3:7 EtOAc:Hex); ${ }^{1} \mathbf{H}$ NMR (600 $\left.\mathrm{MHz} \mathrm{CDCl}_{3}\right) \delta 7.41(\mathrm{~d}, J=7.2 \mathrm{~Hz}, 2 \mathrm{H}), 7.37(\mathrm{t}, J=7.5 \mathrm{~Hz}, 2 \mathrm{H}), 7.31(\mathrm{t}, J=7.1 \mathrm{~Hz}, 1 \mathrm{H}), 5.03$ $(\mathrm{d}, J=1.9 \mathrm{~Hz}, 1 \mathrm{H}), 4.80(\mathrm{~d}, J=10.8 \mathrm{~Hz}, 1 \mathrm{H}), 4.60(\mathrm{~d}, J=10.8 \mathrm{~Hz}, 1 \mathrm{H}), 4.26(\mathrm{~s}, 1 \mathrm{H}), 3.71(\mathrm{~s}$, $3 \mathrm{H}), 2.88(\mathrm{~d}, J=3.7 \mathrm{~Hz}, 1 \mathrm{H}), 2.72(\mathrm{q}, J=6.9 \mathrm{~Hz}, 1 \mathrm{H}), 2.63-2.55(\mathrm{~m}, 1 \mathrm{H}), 2.41(\mathrm{~d}, J=9.8 \mathrm{~Hz}$ 1H), $2.11-2.03(\mathrm{~m}, 1 \mathrm{H}), 1.91-1.83(\mathrm{~m}, 1 \mathrm{H}), 1.81(\mathrm{~d}, J=15.6 \mathrm{~Hz}, 1 \mathrm{H}), 1.52(\mathrm{dd}, J=9.7,1.5$ $\mathrm{Hz}, 1 \mathrm{H}), 1.42(\mathrm{~d}, J=13.5 \mathrm{~Hz}, 1 \mathrm{H}), 1.37-1.29(\mathrm{~m}, 1 \mathrm{H}), 1.29-1.21(\mathrm{~m}, 1 \mathrm{H}), 1.02(\mathrm{~d}, J=7.2 \mathrm{~Hz}$ $3 \mathrm{H}) ;{ }^{13} \mathbf{C}\left\{{ }^{1} \mathbf{H}\right\}$ NMR $\left(151 \mathrm{MHz}, \mathrm{CDCl}_{3}\right) \delta 219.9,172.5,138.1,128.6,128.0,127.6,86.6,66.8$, 66.4, 56.4, 53.7, 52.1, 50.7, 40.8, 34.8, 31.9, 29.5, 18.2, 7.8; HRMS (ESI) m/z: $[\mathrm{M}+\mathrm{Na}]^{+}$ Calcd for $\mathrm{C}_{21} \mathrm{H}_{26} \mathrm{O}_{5} \mathrm{Na} 358.1673$; Found 358.1674 . Relative stereochemistry was assigned by ${ }^{1} \mathrm{H}$ NOESY (see page S52) and by analogy to 22.
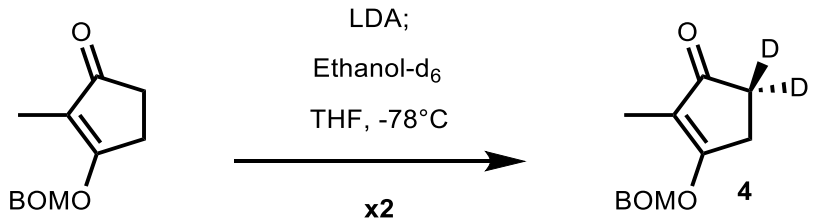


\section{3-((Benzyloxy)methoxy)-2-methylcyclopent-2-en-1-one-5,5-d2 (4):}

To a round bottom flask charged with a stir bar and THF $(3.0 \mathrm{~mL})$ was added diisopropylamine (1.0 mL, $7.2 \mathrm{mmol}, 5.0$ equiv). The solution was cooled to $-78{ }^{\circ} \mathrm{C}$ and $n$-butyl-lithium $(2.5 \mathrm{M}$ in hexanes, $2.6 \mathrm{~mL}, 6.5 \mathrm{mmol} 4.5$ equiv) was added dropwise. The solution was warmed to $0{ }^{\circ} \mathrm{C}$ for 10 minutes, after which it was cooled to $-78^{\circ} \mathrm{C}$ and 3-((benzyloxy)methoxy)-2-methylcyclopent2-en-1-one $\mathrm{e}^{3}(0.34 \mathrm{~g}, 1.4 \mathrm{mmol}, 1.0$ equiv) as a solution in THF (2.0 mL) was added dropwise. The reaction was stirred for 1 hour, after which ethanol- $\mathrm{d}_{6}(0.50 \mathrm{~mL})$ was added rapidly. The reaction was allowed to warm to room temperature and was then diluted with a saturated aqueous solution of ammonium chloride $(5.0 \mathrm{~mL})$. The mixture was transferred to a separatory funnel and the organic layer collected. The aqueous layer was extracted with $\mathrm{CH}_{2} \mathrm{Cl}_{2}(3 \times 2 \mathrm{~mL})$. The combined organic layers were washed with brine $(5 \mathrm{~mL})$, dried with sodium sulfate, and concentrated in vacuo. The residue was subjected to this same procedure once again, after which it was purified by flash column chromatography using $0-60 \%$ EtOAc in hexanes as the eluting solvent to afford 4 as a yellow oil $\left(0.27 \mathrm{~g}, 80 \%\right.$ yield, $65 \%$ deuterium incorporation); $\mathbf{R}_{\boldsymbol{f}}=0.11(1: 1 \mathrm{EtOAc}, \mathrm{UV})$; ${ }^{1} \mathbf{H}$ NMR $\left(600 \mathrm{MHz}, \mathrm{CDCl}_{3}\right) \delta 7.39-7.30(\mathrm{~m}, 5 \mathrm{H}), 5.32(\mathrm{~s}, 2 \mathrm{H}), 4.72(\mathrm{~s}, 2 \mathrm{H}), 2.76-2.67(\mathrm{~m}$, 2H), $2.48-2.39\left(\mathrm{~m}, 0.71 \mathrm{H}\right.$, partially deuterated), $1.65(\mathrm{~s}, 3 \mathrm{H}) ;{ }^{13} \mathbf{C}\left\{{ }^{1} \mathbf{H}\right\} \mathbf{N M R}\left(151 \mathrm{MHz}, \mathrm{CDCl}_{3}\right)$ $\delta$ 205.9, 205.8(8), 205.8(5), 182.3, 182.2(3), 182.1(8), 136.6, 128.7, 128.4, 128.1, 118.3(7), 118.3(5), 91.8, 71.2, 33.7, 33.5, 33.4, 33.3, 24.8(7), 24.7(9), 24.7, 6.1 (See general procedure for explanation of excess carbon peaks); ${ }^{2} \mathbf{H}$ NMR (77 MHz, benzene) $\delta 1.50$ (s, 1D). HRMS (ESI) $\mathrm{m} / \mathrm{z}$ : [undeuterated $\mathrm{M}+\mathrm{Na}^{+}$Calcd for $\mathrm{C}_{14} \mathrm{H}_{16} \mathrm{O}_{3} \mathrm{Na}$ 255.0992; Found 255.0995, m/z: [monodeuterated $\mathrm{M}+\mathrm{Na}]^{+}$Calcd for $\mathrm{C}_{14} \mathrm{H}_{15} \mathrm{DO}_{3} \mathrm{Na}$ 256.1055; Found 256.1054, m/z: [bisdeuterated $\mathrm{M}+\mathrm{Na}]^{+}$Calcd for $\mathrm{C}_{14} \mathrm{H}_{14} \mathrm{D}_{2} \mathrm{O}_{3} \mathrm{Na} 257.1118$; Found 257.1107. 

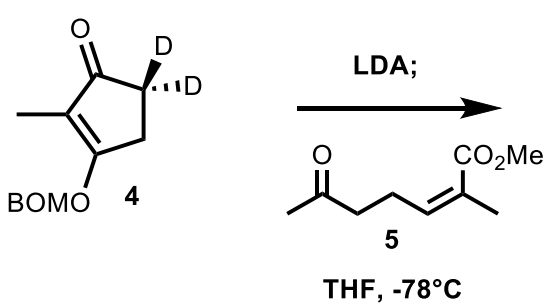

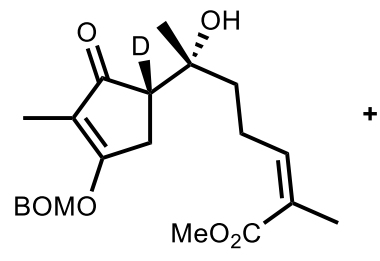

(士) $6 a$

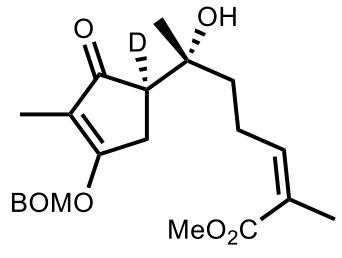

(士) $6 b$

Methyl (Z)-6-(4-((benzyloxy)methoxy)-3-methyl-2-oxocyclopent-3-en-1-yl-1-d)-6-hydroxy2-methylhept-2-enoate (6):

Following general procedure A, 4 ( $89 \mathrm{mg}, 0.38 \mathrm{mmol}, 1.0$ equiv) was deprotonated with LDA (42 $\mathrm{mg}, 0.40 \mathrm{mmol}, 1.1$ equiv) and reacted with methyl $\mathbf{5}^{3}(77 \mathrm{mg}, 0.45 \mathrm{mmol}, 1.2$ equiv) in THF (1.0 $\mathrm{mL}$ ) at $-78^{\circ} \mathrm{C}$. The crude residue was purified by flash column chromatography using $0-40 \%$ EtOAc in hexanes as the eluting solvent to afford $\mathbf{6 a}$ and $\mathbf{6 b}(0.10 \mathrm{~g})$ containing $1.0 \mathrm{wt} \% \mathrm{CH}_{2} \mathrm{Cl}_{2}$ (60\% combined yield of $\mathbf{6 a}$ and $\mathbf{6 b}$, with $62 \%$ and $67 \%$ deuterium incorporation, respectively, $1.7: 1 \mathrm{dr})$

6a:

Clear oil; $\mathbf{R}_{\boldsymbol{f}}=0.42\left(1: 1\right.$ EtOAc:Hex, UV); ${ }^{1} \mathbf{H} \mathbf{N M R}\left(500 \mathrm{MHz}, \mathrm{CDCl}_{3}\right) \delta 7.37-7.27(\mathrm{~m}, 5 \mathrm{H})$, $5.93(\mathrm{t}, J=7.6,1 \mathrm{H}), 5.33(\mathrm{~s}, 2 \mathrm{H}), 4.73(\mathrm{~d}, J=11.8,1 \mathrm{H}), 4.70(\mathrm{~d}, J=12.0,1 \mathrm{H}), 4.37(\mathrm{~s}, 1 \mathrm{H}), 3.70$ (s, 3H), $2.84(\mathrm{~d}, J=17.8,1 \mathrm{H}), 2.66(\mathrm{dd}, J=7.0,2.6,0.33 \mathrm{H}$, partially deuterated $), 2.62-2.45(\mathrm{~m}$, 3H), $1.86(\mathrm{~s}, 3 \mathrm{H}), 1.61(\mathrm{~s}, 3 \mathrm{H}), 1.46-1.36(\mathrm{~m}, 2 \mathrm{H}), 1.15(\mathrm{~s}, 3 \mathrm{H}) ;{ }^{13} \mathbf{C}\left\{{ }^{1} \mathbf{H}\right\} \mathbf{N M R}\left(126 \mathrm{MHz}, \mathrm{CDCl}_{3}\right)$ $\delta$ 208.2(2), 208.2(0), 182.6(3), 182.5(7), 168.5, 143.5, 136.5, 128.7, 128.4, 128.1, 127.0, 118.7(1), 118.6(9), 92.2, 73.9, 73.8, 71.5, 54.3, 51.3, 36.1, 28.6, 28.5, 25.1(3), 25.1(0), 23.9, 20.7, 5.9 (See general procedure for explanation of excess carbon peaks); HRMS (ESI) m/z: [undeuterated M $+\mathrm{Na}]^{+}$Calcd for $\mathrm{C}_{23} \mathrm{H}_{30} \mathrm{O}_{6} \mathrm{Na}$ 425.1935; Found 425.1937, m/z: [deuterated $\left.\mathrm{M}+\mathrm{Na}\right]^{+}$Calcd for $\mathrm{C}_{23} \mathrm{H}_{29} \mathrm{DO}_{6} \mathrm{Na}$ 426.1998; Found 426.1996; Relative stereochemistry was retrospectively deduced 
from the structure of $\mathbf{7 b}$ under the assumption that deuterium migration took place via a suprafacial $[1,5]$ sigmatropic rearrangement.

6b:

Clear oil; $\mathbf{R}_{\boldsymbol{f}}=0.57$ (1:1 EtOAc:Hex, Hanessian's stain); ${ }^{1} \mathbf{H}$ NMR $\left(500 \mathrm{MHz}, \mathrm{CDCl}_{3}\right) \delta 7.38-$ $7.28(\mathrm{~m}, 5 \mathrm{H}), 5.98(\mathrm{t}, J=7.6 \mathrm{~Hz}, 1 \mathrm{H}), 5.35(\mathrm{~d}, J=6.9 \mathrm{~Hz}, 1 \mathrm{H}), 5.32(\mathrm{~d}, J=6.9 \mathrm{~Hz}, 1 \mathrm{H}), 3.70(\mathrm{~s}$, 3H), $2.88(\mathrm{~d}, J=17.8 \mathrm{~Hz}, 1 \mathrm{H}), 2.69$ (dd, $J=7.0,2.5 \mathrm{~Hz}, 0.38 \mathrm{H}$, partially deuterated), $2.67-2.52$ (m, 2H), $2.35(\mathrm{~d}, J=17.8 \mathrm{~Hz}, 1 \mathrm{H}), 1.88(\mathrm{~s}, 3 \mathrm{H}), 1.63(\mathrm{~s}, 3 \mathrm{H}), 1.60-1.45(\mathrm{~m}, 2 \mathrm{H}), 1.00(\mathrm{~s}, 3 \mathrm{H})$; ${ }^{13} \mathbf{C}\left\{{ }^{1} \mathbf{H}\right\}$ NMR (151 MHz, $\left.\mathrm{CDCl}_{3}\right) \delta$ 209.2(7), 209.2(5), 182.8(5), 182.7(9), 168.5, 143.5, 136.5, $128.8,128.5,128.2,127.1,118.7(1), 118.6(9), 92.2,73.7(4), 73.6(9)$ (See general procedure for explanation of excess carbon peaks), 71.6, 51.5, 51.4, 40.4(4), 40.4(3), 28.7, 28.6, 23.8, 22.7, 20.8, 5.9; HRMS (ESI) m/z: [undeuterated $\mathrm{M}+\mathrm{Na}$ ] ${ }^{+} \mathrm{Calcd}$ for $\mathrm{C}_{23} \mathrm{H}_{30} \mathrm{O}_{6} \mathrm{Na}$ 425.1935; Found 425.1915, m/z: [deuterated $\mathrm{M}+\mathrm{Na}$ ] ${ }^{+}$Calcd for $\mathrm{C}_{23} \mathrm{H}_{29} \mathrm{DO}_{6} \mathrm{Na}$ 426.1998; Found 426.2004; Relative stereochemistry was retrospectively deduced from the structure of $\mathbf{7 b}$ under the assumption that deuterium migration took place via a suprafacial $[1,5]$ sigmatropic rearrangement.

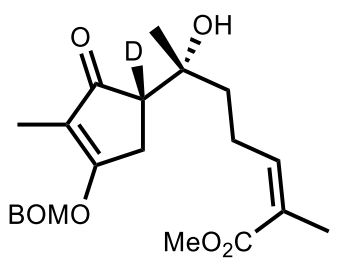

(士) $6 a$

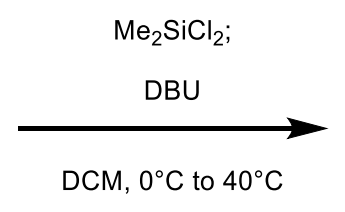

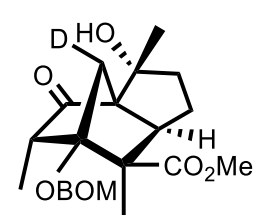

(士) $7 \mathrm{a}$

\section{Epi-methyl 6-((benzyloxy)methoxy)-3-hydroxy-3,5,7-trimethyl-4-oxooctahydro-3a,6- methanoindene-7-carboxylate-8-d (7a):}

Following a modified general procedure 2, 6a (35 mg, $90 \mu \mathrm{mol}, 1.0$ equiv) was reacted with dimethyldichlorosilane (22 $\mu \mathrm{L}, 0.18 \mathrm{mmol}, 2.0$ equiv) and $\mathrm{DBU}$ ( $80 \mu \mathrm{L}, 0.54 \mathrm{mmol}, 6.0$ equiv) in 
$\mathrm{CH}_{2} \mathrm{Cl}_{2}(1.0 \mathrm{~mL})$ from $0{ }^{\circ} \mathrm{C}$ to $40{ }^{\circ} \mathrm{C}$. The product mixture was purified by flash column chromatography using $0-30 \%$ EtOAc in hexanes as the eluting solvent to afford $7 \mathbf{a}$ as a clear oil (3.2 mg 9.0\% yield, 67\% deuterium incorporation, single diastereomer. Note: significant mass balance was lost through cleavage of the BOM group during HF workup): $\mathbf{R}_{\boldsymbol{f}}=0.27$ (1:1 EtOAc:Hex, Hanessian's stain); ${ }^{1} \mathbf{H}$ NMR $\left(600 \mathrm{MHz}, \mathrm{CDCl}_{3}\right) \delta 7.38-7.28(\mathrm{~m}, 5 \mathrm{H}), 5.06(\mathrm{~d}, J=$ $7.7,1 \mathrm{H}), 4.83(\mathrm{~d}, J=7.6,1 \mathrm{H}), 4.68(\mathrm{~d}, J=11.7,1 \mathrm{H}), 4.61(\mathrm{~d}, J=11.7,1 \mathrm{H}), 4.27(\mathrm{~s}, 1 \mathrm{H}), 3.71(\mathrm{~s}$, $3 \mathrm{H}), 3.06-3.01(\mathrm{~m}, 1 \mathrm{H}), 2.96(\mathrm{q}, J=7.1,1 \mathrm{H}), 2.34(\mathrm{dd}, J=10.9,6.0,1 \mathrm{H}), 2.15-2.04(\mathrm{~m}, 1 \mathrm{H})$, $1.95(\mathrm{dd}, J=9.9,2.2,0.33 \mathrm{H}$, partially deuterated $), 1.86-1.81(\mathrm{~m}, 2 \mathrm{H}), 1.52-1.45(\mathrm{~m}, 1 \mathrm{H}), 1.43$ (s, 3H), $1.30(\mathrm{~m}, 6 \mathrm{H}) ;{ }^{13} \mathbf{C}\left\{{ }^{1} \mathbf{H}\right\}$ NMR $\left(151 \mathrm{MHz}, \mathrm{CDCl}_{3}\right) \delta 217.9,174.1,137.5,128.7,128.1(1)$, 128.0(9), 92.5, 89.0(4), 88.9(8), 79.8, 70.2, 68.2, 68.1, 58.2(6), 58.2(5), 57.1(5), 57.1(3), 56.1(5), 56.1(4), 51.9, 40.5, 40.3, 25.1, 24.6, 21.8, 10.3 (See general procedure for explanation of excess carbon peaks); HRMS (ESI) m/z: [undeuterated $\mathrm{M}+\mathrm{Na}{ }^{+}$Calcd for $\mathrm{C}_{23} \mathrm{H}_{30} \mathrm{O}_{6} \mathrm{Na}$ 425.1935; Found 425.1929, m/z: [deuterated $\mathrm{M}+\mathrm{Na}]^{+}$Calcd for $\mathrm{C}_{23} \mathrm{H}_{29} \mathrm{DO}_{6} \mathrm{Na}$ 426.1998; Found 426.1991; stereochemistry of the deuterium atom was established in reference to the protiated analogue of 7a, for which full 2D NMR characterization has been performed. ${ }^{3}$

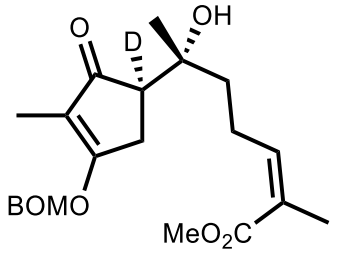

$( \pm) 6 b$

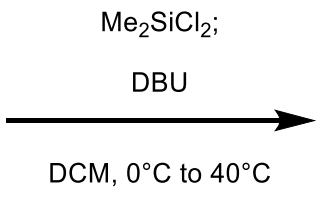

$\mathrm{DCM}, 0^{\circ} \mathrm{C}$ to $40^{\circ} \mathrm{C}$

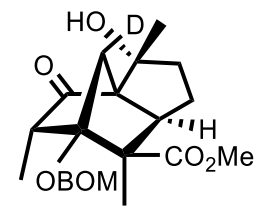

( \pm ) $7 \mathrm{~b}$

Methyl 6-((benzyloxy)methoxy)-3-hydroxy-3,5,7-trimethyl-4-oxooctahydro-3a,6methanoindene-7-carboxylate-8-d (7b): 
Following a modified general procedure $2,6 \mathbf{b}(68 \mathrm{mg}, 0.17 \mathrm{mmol}, 1.0$ equiv) was reacted with dimethyldichlorosilane ( $42 \mu \mathrm{L}, 0.35 \mathrm{mmol}, 2.0$ equiv) and $\mathrm{DBU}(0.15 \mathrm{~mL}, 1.0 \mathrm{mmol}, 6.0$ equiv) in $\mathrm{CH}_{2} \mathrm{Cl}_{2}(2.0 \mathrm{~mL})$ from $0{ }^{\circ} \mathrm{C}$ to $40{ }^{\circ} \mathrm{C}$. The product mixture was purified by flash column chromatography using $0-30 \%$ EtOAc in hexanes as the eluting solvent to afford $7 \mathbf{b}$ as a clear oil (5.0 mg, 7\% yield, 50\% deuterium incorporation, single diastereomer. Note: significant mass balance was lost through cleavage of the BOM group during HF workup): $\mathbf{R}_{\boldsymbol{f}}=0.27$ (1:4 EtOAc:Hex, Hanessian's stain); ${ }^{1} \mathbf{H}$ NMR $\left(600 \mathrm{MHz}, \mathrm{CDCl}_{3}\right) \delta 7.39-7.28(\mathrm{~m}, 5 \mathrm{H}), 5.06(\mathrm{~d}, J=$ $7.5,1 \mathrm{H}), 4.83(\mathrm{~d}, J=7.5,1 \mathrm{H}), 4.68(\mathrm{~d}, J=11.6,1 \mathrm{H}), 4.61(\mathrm{~d}, J=11.6,1 \mathrm{H}), 4.27(\mathrm{~s}, 1 \mathrm{H}), 3.71(\mathrm{~s}$, $3 \mathrm{H}), 3.04(\mathrm{~d}, J=9.9,0.50 \mathrm{H}$, partially deuterated), $2.96(\mathrm{q}, J=6.5,1 \mathrm{H}), 2.34(\mathrm{dd}, J=9.6,6.0,1 \mathrm{H})$, $2.16-2.02(\mathrm{~m}, 1 \mathrm{H}), 1.99-1.92(\mathrm{~m}, 1 \mathrm{H}), 1.88-1.77(\mathrm{~m}, 2 \mathrm{H}), 1.53-1.45(\mathrm{~m}, 1 \mathrm{H}), 1.42(\mathrm{~s}, 3 \mathrm{H})$, $1.30(\mathrm{~m}, 6 \mathrm{H}) ;{ }^{13} \mathbf{C}\left\{{ }^{1} \mathbf{H}\right\} \mathbf{N M R}\left(151 \mathrm{MHz}, \mathrm{CDCl}_{3}\right) \delta 217.9,174.1,137.5,128.7,128.1(1), 128.0(8)$, 92.5, 89.0(4), 88.9(7), 79.7(6), 79.7(5), 70.2, 68.2, 68.1, 58.3, 57.1(5), 57.1(2), 56.2, 51.9, 40.6, 40.3, 25.1, 24.6, 21.8, 10.3 (See general procedure for explanation of excess carbon peaks); HRMS (ESI) $\mathrm{m} / \mathrm{z}$ : [undeuterated $\mathrm{M}+\mathrm{Na}]^{+}$Calcd for $\mathrm{C}_{23} \mathrm{H}_{30} \mathrm{O}_{6} \mathrm{Na}$ 425.1935; Found 425.1951, m/z: [deuterated $\mathrm{M}+\mathrm{Na}]^{+}$Calcd for $\mathrm{C}_{23} \mathrm{H}_{29} \mathrm{DO}_{6} 426.1998$; Found 426.1990; stereochemistry of the deuterium atom was established in reference to the protiated analogue of $\mathbf{7 b}$, for which full $2 \mathrm{D}$ NMR characterization has been performed. ${ }^{3}$

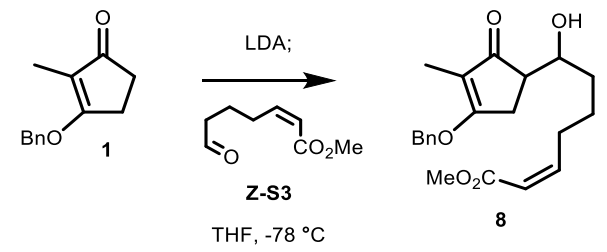

Methyl (Z)-7-(4-(benzyloxy)-3-methyl-2-oxocyclopent-3-en-1-yl)-7-hydroxyhept-2-enoate (8): 
Following general procedure A, 1 ( $0.33 \mathrm{~g}, 1.65 \mathrm{mmol}, 1.0$ equiv) was deprotonated with LDA (0.19 g, $1.8 \mathrm{mmol}, 1.1$ equiv) and reacted with Z-S3 (0.31 mL, $2.0 \mathrm{mmol}, 1.2$ equiv) in THF (4.0 $\mathrm{mL}$ ) at $-78^{\circ} \mathrm{C}$. The product mixture was purified by flash column chromatography using $0-$ $50 \%$ EtOAc in hexanes as the eluting solvent to afford $\mathbf{8}$ as a yellow oil $(0.25 \mathrm{~g}, 43 \%$ yield, $1.6: 1$ dr):

Major Diastereomer:

$\mathbf{R}_{\boldsymbol{f}}=0.46\left(3: 2\right.$ EtOAc:Hex, UV); ${ }^{1} \mathbf{H}$ NMR $\left(600 \mathrm{MHz}, \mathrm{CDCl}_{3}\right) \delta 7.45-7.40(\mathrm{~m}, 2 \mathrm{H}), 7.40-7.34$ (m, 3H), $6.24(\mathrm{dt}, J=11.5,7.6 \mathrm{~Hz}, 1 \mathrm{H}), 5.79(\mathrm{~d}, J=11.4 \mathrm{~Hz}, 1 \mathrm{H}), 5.25(\mathrm{~s}, 2 \mathrm{H}), 4.80(\mathrm{~s}, 1 \mathrm{H})$, 3.70 (s, 3H), 3.65 (td, $J=9.3,2.7 \mathrm{~Hz}, 1 \mathrm{H}), 2.79$ (dd, $J=17.3,6.5 \mathrm{~Hz}, 1 \mathrm{H}), 2.72-2.64$ (m, 2H), $2.46(\mathrm{t}, J=7.1 \mathrm{~Hz}, 1 \mathrm{H}), 2.32(\mathrm{~d}, J=17.9 \mathrm{~Hz}, 1 \mathrm{H}), 1.75-1.69(\mathrm{~m}, 1 \mathrm{H}), 1.68(\mathrm{~s}, 3 \mathrm{H}), 1.65-1.59$ (m, 1H), $1.53-1.45(\mathrm{~m}, 2 \mathrm{H}) ;{ }^{13} \mathbf{C}\left\{{ }^{1} \mathbf{H}\right\} \mathbf{N M R}\left(151 \mathrm{MHz}, \mathrm{CDCl}_{3}\right) \delta 208.5,183.8,167.1,150.8$, 135.7, 129.1, 128.8, 127.2, 119.6, 116.4, 72.4, 71.4, 51.2, 48.9, 35.4, 29.1, 28.9, 24.5, 6.1;

HRMS (ESI) m/z: $[\mathrm{M}+\mathrm{Na}]^{+}$Calcd for $\mathrm{C}_{21} \mathrm{H}_{26} \mathrm{O}_{5} \mathrm{Na}$ 381.1673; Found 381.1672.

Minor Diastereomer:

$\mathbf{R}_{f}=0.43\left(3: 2\right.$ EtOAc:Hex, UV); ${ }^{1} \mathbf{H}$ NMR $\left(600 \mathrm{MHz}, \mathrm{CDCl}_{3}\right) \delta 7.43-7.39(\mathrm{~m}, 2 \mathrm{H}), 7.38-7.34$ (m, 3H), $6.23(\mathrm{dt}, J=11.6,7.6 \mathrm{~Hz}, 1 \mathrm{H}), 5.79(\mathrm{~d}, J=11.5 \mathrm{~Hz}, 1 \mathrm{H}), 5.27(\mathrm{~d}, J=12.4 \mathrm{~Hz}, 1 \mathrm{H})$, $5.24(\mathrm{~d}, J=12.4 \mathrm{~Hz}, 1 \mathrm{H}), 4.17(\mathrm{~d}, J=3.5 \mathrm{~Hz}, 1 \mathrm{H}), 3.70(\mathrm{~s}, 3 \mathrm{H}), 2.75-2.64(\mathrm{~m}, 3 \mathrm{H}), 2.64-2.58$ $(\mathrm{m}, 2 \mathrm{H}), 2.29(\mathrm{~d}, J=5.5 \mathrm{~Hz}, 1 \mathrm{H}), 1.67(\mathrm{~s}, 3 \mathrm{H}), 1.53-1.38(\mathrm{~m}, 3 \mathrm{H}) ;{ }^{13} \mathbf{C}\left\{{ }^{1} \mathbf{H}\right\} \mathbf{N M R}(151 \mathrm{MHz}$, $\left.\mathrm{CDCl}_{3}\right) \delta 206.6,184.4,167.0,150.4,135.9,129.0,128.7,127.2,119.8,117.2,71.2,70.0,51.2$, 50.5, 34.1, 28.8, 26.7, 25.6, 6.2; HRMS (ESI) m/z: $[\mathrm{M}+\mathrm{Na}]^{+}$Calcd for $\mathrm{C}_{21} \mathrm{H}_{26} \mathrm{O}_{5} \mathrm{Na}$ 381.1673; Found 381.1668 . 


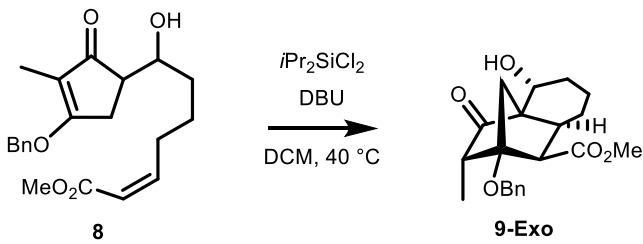

Methyl-2-(benzyloxy)-5-hydroxy-3-methyl-4-oxooctahydro-2H-2,4a-methanonaphthalene1-carboxylate (9-Exo):

Following general procedure $\mathrm{B}, \mathbf{8}(0.25 \mathrm{~g}, 0.71 \mathrm{mmol}, 1.0$ equiv $)$ was reacted with diisopropyldichlorosilane (0.25 mL, $1.4 \mathrm{mmol}, 2.0$ equiv) and $\mathrm{DBU}$ (0.63 mL, $4.2 \mathrm{mmol}, 6.0$ equiv) in $\mathrm{CH}_{2} \mathrm{Cl}_{2}(10 \mathrm{~mL})$ from 0 to $40^{\circ} \mathrm{C}$. The product mixture was purified by flash column chromatography using $0-30 \%$ EtOAc in hexanes as the eluting solvent to afford 9-exo as a clear oil (195 mg 77\% yield, single diastereomer): $\mathbf{R}_{\boldsymbol{f}}=0.48$ (3:7 EtOAc:Hex, Hanessian's stain); ${ }^{1} \mathbf{H}$ NMR $\left(600 \mathrm{MHz}, \mathrm{CDCl}_{3}\right) \delta 7.32$ - $7.17(\mathrm{~m}, 5 \mathrm{H}), 4.99(\mathrm{~s}, 1 \mathrm{H}), 4.58(\mathrm{~d}, J=11.3 \mathrm{~Hz}$ $1 \mathrm{H}), 4.47(\mathrm{~d}, J=11.4 \mathrm{~Hz}, 1 \mathrm{H}), 4.21(\mathrm{~s}, 1 \mathrm{H}), 3.61(\mathrm{~s}, 3 \mathrm{H}), 3.07(\mathrm{~d}, J=9.2 \mathrm{~Hz}, 1 \mathrm{H}), 2.77(\mathrm{~d}, J=$ $10.0 \mathrm{~Hz}, 1 \mathrm{H}), 2.60(\mathrm{q}, J=6.9 \mathrm{~Hz}, 1 \mathrm{H}), 2.19-2.06(\mathrm{~m}, 1 \mathrm{H}), 1.76-1.61(\mathrm{~m}, 4 \mathrm{H}), 1.48-1.40(\mathrm{~m}$, 1H), $1.40-1.34(\mathrm{~m}, 1 \mathrm{H}), 1.32-1.24(\mathrm{~m}, 1 \mathrm{H}), 1.04(\mathrm{~d}, J=7.0 \mathrm{~Hz}, 3 \mathrm{H}) ;{ }^{13} \mathbf{C}\left\{{ }^{1} \mathbf{H}\right\} \mathbf{N M R}(126$ $\left.\mathrm{MHz}, \mathrm{CDCl}_{3}\right) \delta 220.9,171.0,138.3,128.5,127.8,127.4,85.6,68.0,67.0,57.7,51.5,51.4,47.4$, 37.2, 35.8, 29.6, 25.2, 18.0, 7.8; HRMS (ESI) m/z: $[\mathrm{M}+\mathrm{Na}]^{+}$Calcd for $\mathrm{C}_{21} \mathrm{H}_{26} \mathrm{O}_{5} \mathrm{Na} 381.1673$; Found 381.1669. The $\sim 10 \mathrm{~Hz}$ coupling constant between the adjacent methine protons established the relative stereochemistry of the ester. Remaining stereochemistry was established in analogy with 2-endo.

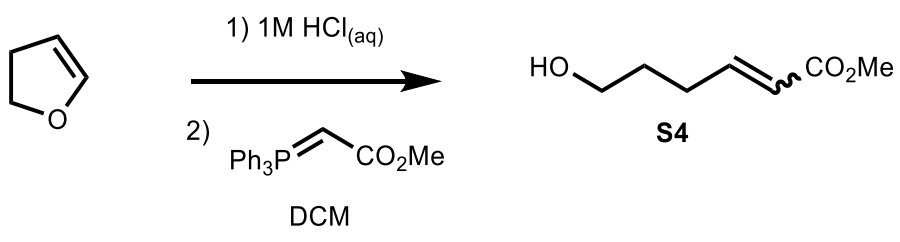




\section{Methyl 6-hydroxyhex-2-enoate (S4):}

No precaution was taken to exclude air or water from the reaction. A round bottom flask charged with 2,3-dihydro-2H-furan (1.9 mL, $25 \mathrm{mmol}, 1.0$ equiv) was cooled to $0{ }^{\circ} \mathrm{C}$ and an aqueous solution of $\mathrm{HCl}(5.0 \mathrm{~mL}, 0.2 \underline{\mathrm{M}})$ was added slowly. This mixture was stirred at $0{ }^{\circ} \mathrm{C}$ for 15 minutes and then 1 hour at room temperature. The crude reaction mixture was extracted with $\mathrm{CH}_{2} \mathrm{Cl}_{2}(3 \times 5 \mathrm{~mL})$. The combined organic layers were washed with brine $(5 \mathrm{~mL})$, dried over sodium sulfate, and concentrated in vacuo. The product mixture was then dissolved in THF (60 $\mathrm{mL}$ ) and transferred to a round bottom flask charged with a magnetic stir bar and reflux condenser. Methyl 2-(triphenyl- $\lambda^{5}$-phosphaneylidene)acetate (10 g, $30 \mathrm{mmol}, 1.2$ equiv) was added and the reaction was refluxed for 13 hours. After this time, the crude reaction was concentrated in vacuo and diluted with a 7:3 diethyl ether : pentanes solution. This mixture was stirred for 45 minutes, during which time triphenylphosphine oxide precipitated as a white solid. The mixture was filtered to remove the phosphine oxide and the filtrate was concentrated in vасио. The crude residue was purified by flash column chromatography using $0-50 \%$ EtOAc in hexanes as the eluting solvent to afford clear oil S4 as an inseparable mixture of olefin isomers (1.2 g, 34\% yield, 20:1 E:Z); all spectral data are consistent with those reported. ${ }^{4} \mathbf{H}$ NMR (500 $\left.\mathrm{MHz}_{\mathrm{CDCl}}\right) \delta 6.96(\mathrm{dt}, J=15.5,7.0 \mathrm{~Hz}, 1 \mathrm{H}), 5.83(\mathrm{~d}, J=15.6 \mathrm{~Hz}, 1 \mathrm{H}), 3.68(\mathrm{~d}, J=16.3 \mathrm{~Hz}$, $3 \mathrm{H}), 3.64(\mathrm{t}, J=6.4 \mathrm{~Hz}, 2 \mathrm{H}), 2.34-2.22(\mathrm{~m}, 2 \mathrm{H}), 1.89(\mathrm{bs}, 1 \mathrm{H}), 1.76-1.63(\mathrm{~m}, 2 \mathrm{H})$. Only E isomer peaks are reported
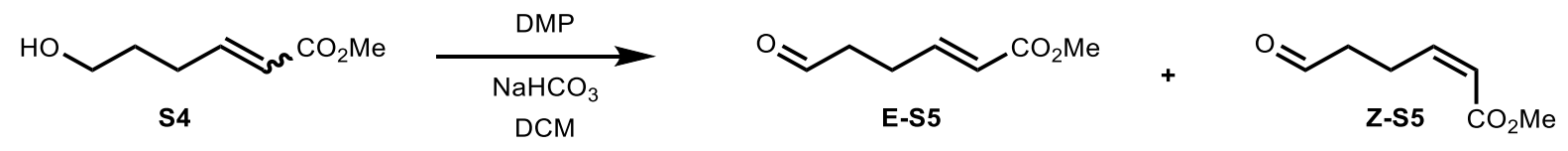

\section{Methyl (E)-6-hydroxyhex-2-enoate (S5)}


Following general procedure C, S4 (1.2 g, $8.5 \mathrm{mmol}, 1.0)$ was reacted with Dess-Martin periodinane (5.4 g, $13 \mathrm{mmol}, 1.5$ equiv) and sodium bicarbonate ( $7.1 \mathrm{~g}, 85 \mathrm{mmol}, 10$ equiv) in $\mathrm{CH}_{2} \mathrm{Cl}_{2}(85 \mathrm{~mL})$. The product mixture was purified by flash column chromatography using $0-$ $30 \%$ EtOAc in hexanes as the eluting solvent to afford E-S5 as a pale yellow oil $(0.70 \mathrm{~g}) 58 \%$ yield).

\section{E-S5}

All spectral data are consistent with those reported; ${ }^{1} \mathbf{H}$ NMR $\left(500 \mathrm{MHz}, \mathrm{CDCl}_{3}\right) \delta 9.78(\mathrm{~s}, 1 \mathrm{H})$, $6.93(\mathrm{dt}, J=15.5,6.5 \mathrm{~Hz}, 1 \mathrm{H}), 5.84(\mathrm{~d}, J=15.7 \mathrm{~Hz}, 1 \mathrm{H}), 3.71(\mathrm{~s}, 3 \mathrm{H}), 2.62(\mathrm{t}, J=7.1 \mathrm{~Hz}, 2 \mathrm{H})$, $2.52(\mathrm{q}, J=6.9 \mathrm{~Hz}, 2 \mathrm{H})$.

Z-S5 was not isolated.
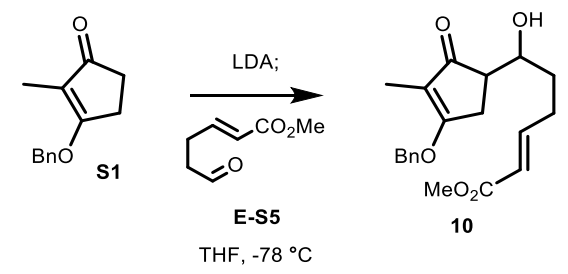

Methyl (E)-6-(4-(benzyloxy)-3-methyl-2-oxocyclopent-3-en-1-yl)-6-hydroxyhex-2-enoate (10):

Following general procedure A, S1 (0.83 g, $4.1 \mathrm{mmol}, 1.0$ equiv) was deprotonated with LDA (0.48 g, 4.5 mmol, 1.1 equiv) and reacted with E-S5 ( $0.70 \mathrm{~g}, 4.9 \mathrm{mmol}, 1.2$ equiv) in THF (10 $\mathrm{mL})$ at $-78^{\circ} \mathrm{C}$. The product mixture was purified by flash column chromatography using $0-$ $60 \%$ EtOAc in hexanes as the eluting solvent to afford the major diastereomer of $\mathbf{1 0}$ as a yellow oil $(0.25 \mathrm{~g}, 18 \%$ yield $)$. The minor diastereomer was unable to be isolated in acceptable purity: 
$\mathbf{R}_{\boldsymbol{f}}=0.41\left(3: 2\right.$ EtOAc:Hex, UV); ${ }^{1} \mathbf{H}$ NMR $\left(500 \mathrm{MHz}, \mathrm{CDCl}_{3}\right) \delta 7.44-7.31(\mathrm{~m}, 5 \mathrm{H}), 6.96(\mathrm{dt}, J$

$=15.5,6.5 \mathrm{~Hz}, 1 \mathrm{H}), 5.84(\mathrm{~d}, J=15.6 \mathrm{~Hz}, 1 \mathrm{H}), 5.24(\mathrm{~s}, 2 \mathrm{H}), 4.82(\mathrm{~s}, 1 \mathrm{H}), 3.70(\mathrm{~s}, 3 \mathrm{H}), 3.62(\mathrm{~s}$,

$1 \mathrm{H}), 2.78(\mathrm{dd}, J=17.1,6.3 \mathrm{~Hz}, 1 \mathrm{H}), 2.51-2.39(\mathrm{~m}, 2 \mathrm{H}), 2.37-2.25(\mathrm{~m}, 2 \mathrm{H}), 1.66(\mathrm{~s}, 3 \mathrm{H}), 1.62$

$-1.54(\mathrm{~m}, 2 \mathrm{H}) ;{ }^{13} \mathbf{C}\left\{{ }^{1} \mathbf{H}\right\} \mathbf{N M R}\left(126 \mathrm{MHz}, \mathrm{CDCl}_{3}\right) \delta 208.1,183.7,167.2,149.0,135.6,129.0$,

128.8, 127.2, 121.4, 116.3, 71.8, 71.4, 51.5, 48.9, 34.0, 28.9, 27.8, 6.1; HRMS (ESI) m/z: $[\mathrm{M}+$ $\mathrm{Na}]^{+}$Calcd for $\mathrm{C}_{20} \mathrm{H}_{24} \mathrm{O}_{5} \mathrm{Na}$ 367.1516; Found 367.1532.

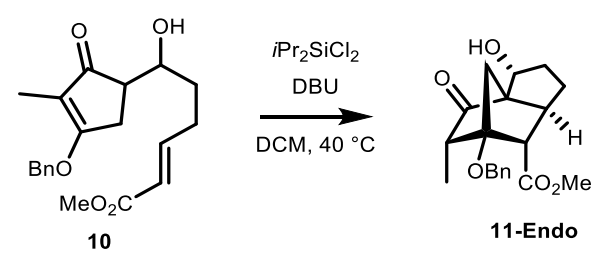

\section{Methyl-6-(benzyloxy)-3-hydroxy-5-methyl-4-oxooctahydro-3a,6-methanoindene-7- carboxylate (11-Endo):}

This reaction was performed with diastereopure 10, consisting only of the major diastereomer of the previous reaction. Following general procedure B, $10(0.25 \mathrm{~g}, 0.72 \mathrm{mmol}, 1.0$ equiv) was reacted with diisopropyldichlorosilane $(0.26 \mathrm{~mL}, 1.5 \mathrm{mmol}, 2.0$ equiv) and $\mathrm{DBU}(0.65 \mathrm{~mL}, 4.3$ mmol, 6.0 equiv) in $\mathrm{CH}_{2} \mathrm{Cl}_{2}(10 \mathrm{~mL})$ from 0 to $40{ }^{\circ} \mathrm{C}$. The product mixture was purified by flash column chromatography using $0-30 \%$ EtOAc in hexanes as the eluting solvent to afford to afford 11-Endo as a yellow oil (139 $\mathrm{mg} 56 \%$ yield, single diastereomer): $\mathbf{R}_{\boldsymbol{f}}=0.53$ (2:3 EtOAc:Hex, Hanessian's stain); ${ }^{1} \mathbf{H}$ NMR $\left(500 \mathrm{MHz}, \mathrm{CDCl}_{3}\right) \delta 7.44-7.34(\mathrm{~m}, 4 \mathrm{H}), 7.34-7.28$ (m, 1H), $4.86(\mathrm{~d}, J=10.7 \mathrm{~Hz}, 1 \mathrm{H}), 4.58(\mathrm{~d}, J=10.7 \mathrm{~Hz}, 1 \mathrm{H}), 4.45(\mathrm{~d}, J=4.2 \mathrm{~Hz}, 1 \mathrm{H}), 4.17(\mathrm{~s}$, 1H), $3.74(\mathrm{~s}, 3 \mathrm{H}), 3.15(\mathrm{~d}, J=6.1 \mathrm{~Hz}, 1 \mathrm{H}), 3.09-3.01(\mathrm{~m}, 1 \mathrm{H}), 2.67$ (q, $J=7.3 \mathrm{~Hz}, 1 \mathrm{H}), 2.53-$ $2.39(\mathrm{~m}, 1 \mathrm{H}), 2.13-2.03(\mathrm{~m}, 1 \mathrm{H}), 2.02(\mathrm{~d}, J=9.7 \mathrm{~Hz}, 1 \mathrm{H}), 1.92(\mathrm{ddd}, J=13.8,9.0,4.4 \mathrm{~Hz}, 1 \mathrm{H})$, $1.67(\mathrm{dd}, J=9.7,1.4 \mathrm{~Hz}, 1 \mathrm{H}), 1.54-1.44(\mathrm{~m}, 1 \mathrm{H}), 1.07(\mathrm{~d}, J=7.4 \mathrm{~Hz}, 3 \mathrm{H}) ;{ }^{13} \mathbf{C}\left\{{ }^{1} \mathbf{H}\right\} \mathbf{N M R}(126$ 
$\left.\mathrm{MHz}, \mathrm{CDCl}_{3}\right) \delta 216.1,172.2,138.1,128.6,127.9,127.6,87.6,73.8,66.3,65.1,54.2,52.1,50.8$, 43.7, 42.0, 35.5, 29.8, 8.4; HRMS (ESI) m/z: $[\mathrm{M}+\mathrm{Na}]^{+}$Calcd for $\mathrm{C}_{20} \mathrm{H}_{24} \mathrm{O}_{5} \mathrm{Na} 367.1516$; Found 367.1518. Relative stereochemistry was established in analogy with 2-endo.
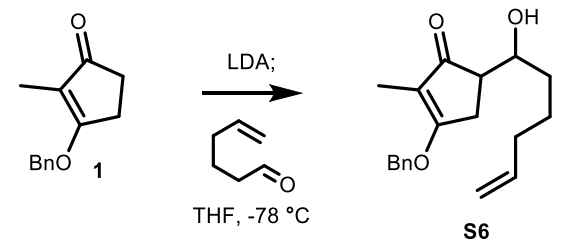

\section{3-(benzyloxy)-5-(1-hydroxyhex-5-en-1-yl)-2-methylcyclopent-2-en-1-one (S6)}

Following general procedure A, 1 ( $1.0 \mathrm{~g}, 5.0 \mathrm{mmol}, 1.0$ equiv) was deprotonated with LDA (0.58 $\mathrm{g}, 5.4 \mathrm{mmol}, 1.1$ equiv) and reacted with hex-5-en-1-al ( $0.58 \mathrm{~g}, 5.9 \mathrm{mmol}, 1.2$ equiv) in THF (12 $\mathrm{mL}$ ) at $-78^{\circ} \mathrm{C}$. The product mixture was purified by flash column chromatography using $0-$ $35 \%$ EtOAc in hexanes as the eluting solvent to afford yellow oil $\mathbf{S 6}$ as an inseparable mixture of diastereomers (635 mg, 38\% yield, 1:1.2 dr): $\mathbf{R}_{\boldsymbol{f}}=0.32$ (2:3 EtOAc:Hex, UV); ${ }^{1} \mathbf{H}$ NMR (600 $\left.\mathrm{MHz}, \mathrm{CDCl}_{3}\right) \delta 7.44-7.33(\mathrm{~m}, 5 \mathrm{H}), 5.84-5.74(\mathrm{~m}, 1 \mathrm{H}), 5.28-5.21(\mathrm{~m}, 2 \mathrm{H}), 5.03-4.97(\mathrm{~m}$, 1H), $4.97-4.93(\mathrm{~m}, 1 \mathrm{H}), 4.19-4.14(\mathrm{~m}, 0.46 \mathrm{H}), 3.66-3.59(\mathrm{~m}, 0.55 \mathrm{H}), 2.79(\mathrm{dd}, J=17.0,6.6$ $\mathrm{Hz}, 0.57 \mathrm{H}), 2.72-2.62(\mathrm{~m}, 1 \mathrm{H}), 2.59(\mathrm{dt}, J=6.2,2.9 \mathrm{~Hz}, 0.48 \mathrm{H}), 2.46(\mathrm{ddd}, J=9.6,7.1,2.8 \mathrm{~Hz}$, $0.57 \mathrm{H}), 2.30(\mathrm{~d}, J=17.4 \mathrm{~Hz}, 0.58 \mathrm{H}), 2.14-2.05(\mathrm{~m}, 2 \mathrm{H}), 1.69-1.65(\mathrm{~m}, 3 \mathrm{H}), 1.65-1.57(\mathrm{~m}$, 1H), $1.54-1.36(\mathrm{~m}, 3 \mathrm{H}) ;{ }^{13} \mathbf{C}\left\{{ }^{1} \mathbf{H}\right\} \mathbf{N M R}\left(151 \mathrm{MHz}, \mathrm{CDCl}_{3}\right) \delta$ 208.5, 206.7, 184.4, 183.7, 138.8, $138.7,135.9,135.7,129.0(2), 129.0(0), 128.8,128.7,127.2(1), 127.1(6), 117.2,116.3,114.9$, $114.7,72.6,71.4,71.2,70.1,50.5,49.0,35.1,34.1,33.7,33.7,29.1,26.6,25.4,24.3,6.2,6.1$; HRMS (ESI) m/z: [M + Na $]^{+}$Calcd for $\mathrm{C}_{19} \mathrm{H}_{24} \mathrm{O}_{3} \mathrm{Na}$ 323.1618; Found 323.1610. 


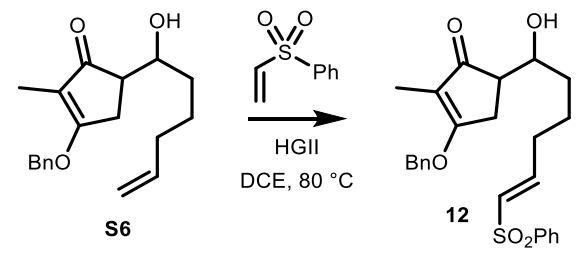

(E)-3-(benzyloxy)-5-(1-hydroxy-6-(phenylsulfonyl)hex-5-en-1-yl)-2-methylcyclopent-2-en-1one (12):

To a scintillation vial equipped with a stir bar and charged with $\mathrm{ClCH}_{2} \mathrm{CH}_{2} \mathrm{Cl}(10 \mathrm{~mL})$ was added S6 (0.55 g, $1.8 \mathrm{mmol}, 1.0$ equiv), phenyl vinyl sulfone ( $1.0 \mathrm{~g}, 6.0 \mathrm{mmol}, 3.3$ equiv), and Hoveyda-Grubbs $2^{\text {nd }}$ Generation catalyst (100 mg, $0.18 \mathrm{mmol}, 10 \%$ loading). The vial was sealed and the mixture was heated to $80{ }^{\circ} \mathrm{C}$ for 13 hours. The reaction mixture was transferred to a separatory funnel and washed with an aqueous solution of tris(hydroxymethyl)phosphine (10 $\mathrm{mL}, 0.25 \mathrm{M}) .{ }^{6}$ The aqueous layer was extracted with $\mathrm{CH}_{2} \mathrm{Cl}_{2}(3 \times 5 \mathrm{~mL})$, after which the combined organic layers were dried over sodium sulfate and concentrated in vacuo. The crude residue was purified by flash column chromatography using $0-70 \%$ EtOAc in hexanes as the eluting solvent to afford 12 as a yellow oil (393 $\mathrm{mg}, 49 \%$ yield, 1.2:1 dr):

Major Diastereomer:

$\mathbf{R}_{\boldsymbol{f}}=0.54\left(7: 3\right.$ EtOAc:Hex, UV); ${ }^{1} \mathbf{H} \mathbf{N M R}\left(600 \mathrm{MHz}, \mathrm{CDCl}_{3}\right) \delta 7.87(\mathrm{~d}, J=7.8 \mathrm{~Hz}, 2 \mathrm{H}), 7.60(\mathrm{t}$, $J=7.3 \mathrm{~Hz}, 1 \mathrm{H}), 7.53(\mathrm{t}, J=7.6 \mathrm{~Hz}, 2 \mathrm{H}), 7.44-7.40(\mathrm{~m}, 2 \mathrm{H}), 7.37(\mathrm{t}, J=8.2 \mathrm{~Hz}, 3 \mathrm{H}), 6.99(\mathrm{dt}, J$ $=15.0,6.6 \mathrm{~Hz}, 1 \mathrm{H}), 6.34(\mathrm{~d}, J=15.1 \mathrm{~Hz}, 1 \mathrm{H}), 5.29-5.23(\mathrm{~m}, 2 \mathrm{H}), 4.86(\mathrm{bs}, 1 \mathrm{H}), 3.60(\mathrm{td}, J=$ 8.4, 2.4 Hz, 1H), $2.78(\mathrm{dd}, J=17.1,7.0 \mathrm{~Hz}, 1 \mathrm{H}), 2.42(\mathrm{ddd}, J=10.2,7.2,2.4 \mathrm{~Hz}, 1 \mathrm{H}), 2.32-$ $2.23(\mathrm{~m}, 3 \mathrm{H}), 1.77-1.70(\mathrm{~m}, 1 \mathrm{H}), 1.67(\mathrm{~s}, 3 \mathrm{H}), 1.63-1.56(\mathrm{~m}, 2 \mathrm{H}), 1.52-1.40(\mathrm{~m}, 2 \mathrm{H})$; ${ }^{13} \mathbf{C}\left\{{ }^{1} \mathbf{H}\right\}$ NMR $\left(151 \mathrm{MHz}, \mathrm{CDCl}_{3}\right) \delta 208.4,183.8,146.8,140.7,135.7,133.4,130.9,129.4$ 
129.1, 128.8, 127.7, 127.2, 116.3, 72.3, 71.5, 48.8, 34.9, 31.3, 29.1, 23.1, 6.1; HRMS (ESI) m/z:

$[\mathrm{M}+\mathrm{Na}]^{+}$Calcd for $\mathrm{C}_{25} \mathrm{H}_{28} \mathrm{O}_{5} \mathrm{SNa}$ 463.1550; Found 463.1552.

More polar Diastereomer:

$\mathbf{R}_{f}=0.45\left(7: 3\right.$ EtOAc:Hex, UV); ${ }^{1} \mathbf{H}$ NMR $\left(600 \mathrm{MHz}, \mathrm{CDCl}_{3}\right) \delta 7.87(\mathrm{~d}, J=7.7 \mathrm{~Hz}, 2 \mathrm{H}), 7.60(\mathrm{t}$, $J=7.3 \mathrm{~Hz}, 1 \mathrm{H}), 7.53$ (t, $J=7.6 \mathrm{~Hz}, 2 \mathrm{H}), 7.42-7.33(\mathrm{~m}, 5 \mathrm{H}), 6.97(\mathrm{dt}, J=15.0,6.6 \mathrm{~Hz}, 1 \mathrm{H})$,

$6.33(\mathrm{~d}, J=15.1 \mathrm{~Hz}, 1 \mathrm{H}), 5.28-5.22(\mathrm{~m}, 2 \mathrm{H}), 4.11(\mathrm{~d}, J=8.1 \mathrm{~Hz}, 1 \mathrm{H}), 2.64(\mathrm{~s}, 2 \mathrm{H}), 2.58-2.54$

(m, 1H), $2.31-2.22(\mathrm{~m}, 2 \mathrm{H}), 1.81-1.67(\mathrm{~m}, 2 \mathrm{H}), 1.64(\mathrm{~s}, 3 \mathrm{H}), 1.55-1.33(\mathrm{~m}, 3 \mathrm{H}) ;{ }^{13} \mathbf{C}\left\{{ }^{1} \mathbf{H}\right\}$

NMR $\left(151 \mathrm{MHz}, \mathrm{CDCl}_{3}\right) \delta 206.5,184.7,146.7,140.7,135.9,133.4,130.9,129.4,129.0,128.7$,

127.7, 127.2, 117.2, 71.3, 69.7, 50.5, 33.7, 31.3, 26.7, 24.3, 6.2; HRMS (ESI) m/z: $[\mathrm{M}+\mathrm{Na}]^{+}$

Calcd for $\mathrm{C}_{25} \mathrm{H}_{28} \mathrm{O}_{5} \mathrm{SNa} 463.1550$; Found 463.1572.

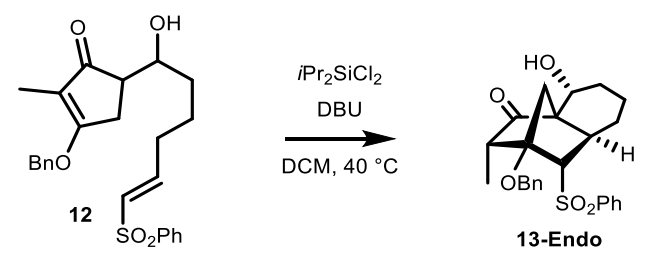

2-(benzyloxy)-5-hydroxy-3-methyl-1-(phenylsulfonyl)octahydro-4H-2,4amethanonaphthalen-4-one (13-Endo):

Following general procedure B, 12 ( $0.39 \mathrm{~g}, 0.89 \mathrm{mmol}, 1.0$ equiv $)$ was reacted with diisopropyldichlorosilane ( $0.32 \mathrm{~mL}, 1.8 \mathrm{mmol}, 2.0$ equiv) and DBU ( $0.80 \mathrm{~mL}, 5.4 \mathrm{mmol}, 6.0$ equiv) in $\mathrm{CH}_{2} \mathrm{Cl}_{2}(10 \mathrm{~mL})$ from 0 to $40{ }^{\circ} \mathrm{C}$. The product mixture was purified by flash column chromatography using $0-35 \%$ EtOAc in hexanes as the eluting solvent to afford $\mathbf{1 3}$ as a foamy white solid (212 mg 54\% yield, single diastereomer): $\mathbf{R}_{f}=0.53$ (2:3 EtOAc:Hex, Hanessian's stain); ${ }^{1} \mathbf{H}$ NMR $\left(500 \mathrm{MHz}, \mathrm{CDCl}_{3}\right) \delta 7.89(\mathrm{~d}, J=7.7 \mathrm{~Hz}, 2 \mathrm{H}), 7.62(\mathrm{t}, J=7.4 \mathrm{~Hz}, 1 \mathrm{H}), 7.53$ (t, $J$ $=7.7 \mathrm{~Hz}, 2 \mathrm{H}), 7.38-7.30(\mathrm{~m}, 5 \mathrm{H}), 5.02(\mathrm{~d}, J=2.0 \mathrm{~Hz}, 1 \mathrm{H}), 4.71-4.61(\mathrm{~m}, 2 \mathrm{H}), 4.23(\mathrm{~s}, 1 \mathrm{H})$, 
$3.52(\mathrm{~d}, J=4.8 \mathrm{~Hz}, 1 \mathrm{H}), 2.97(\mathrm{q}, J=7.0 \mathrm{~Hz}, 1 \mathrm{H}), 2.53(\mathrm{dt}, J=10.9,4.2 \mathrm{~Hz}, 1 \mathrm{H}), 2.26(\mathrm{~d}, J=$ $10.0 \mathrm{~Hz}, 1 \mathrm{H}), 1.75-1.66(\mathrm{~m}, 3 \mathrm{H}), 1.58(\mathrm{~d}, J=7.1 \mathrm{~Hz}, 3 \mathrm{H}), 1.29-1.16(\mathrm{~m}, 3 \mathrm{H}), 1.01(\mathrm{qd}, J=$ 12.0, $2.5 \mathrm{~Hz}, 1 \mathrm{H}) ;{ }^{13} \mathbf{C}\left\{{ }^{1} \mathbf{H}\right\} \mathbf{N M R}\left(151 \mathrm{MHz}, \mathrm{CDCl}_{3}\right) \delta 218.2,141.3,137.5,133.9,129.5,128.7$, 128.2, 128.1, 127.6, 89.1, 72.6, 67.2, 66.7, 56.5, 53.7, 42.0, 36.3, 31.2, 28.9, 17.9, 9.1; HRMS (ESI) m/z: $[\mathrm{M}+\mathrm{Na}]^{+}$Calcd for $\mathrm{C}_{25} \mathrm{H}_{28} \mathrm{O}_{5} \mathrm{SNa}$ 463.1550; Found 463.1560. Relative stereochemistry was established in analogy with 2-endo.

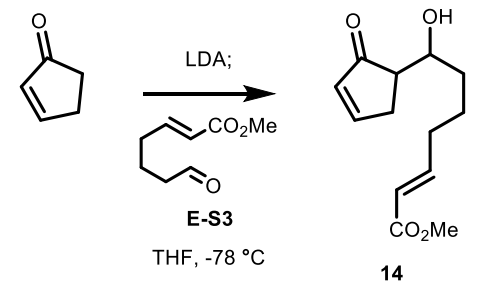

\section{Methyl (E)-7-hydroxy-7-(2-oxocyclopent-3-en-1-yl)hept-2-enoate (14):}

Following general procedure A, 2-cyclopenten-1-one ( $0.14 \mathrm{~mL}, 1.7 \mathrm{mmol}, 1.0$ equiv) was deprotonated with LDA $(0.20 \mathrm{~g}, 1.9 \mathrm{mmol}, 1.1$ equiv) and reacted with E-S3 (0.29 g, $2.0 \mathrm{mmol}$, 1.2 equiv) in THF $(4.0 \mathrm{~mL})$ at $-78^{\circ} \mathrm{C}$. The product mixture was purified by flash column chromatography using $0-50 \%$ EtOAc in hexanes as the eluting solvent to afford the major diastereomer of 14 as a clear oil (0.13 g 34\% yield).

Major diastereomer:

$\mathbf{R}_{\boldsymbol{f}}=0.33\left(3: 2\right.$ EtOAc:Hex, $\left.\mathrm{KMnO}_{4}\right) ;{ }^{1} \mathbf{H} \mathbf{N M R}\left(600 \mathrm{MHz}, \mathrm{CDCl}_{3}\right) \delta 7.75(\mathrm{~d}, J=2.1 \mathrm{~Hz}, 1 \mathrm{H})$, $7.01-6.91(\mathrm{~m}, 1 \mathrm{H}), 6.21(\mathrm{~s}, 1 \mathrm{H}), 5.83(\mathrm{~d}, J=15.7 \mathrm{~Hz}, 1 \mathrm{H}), 4.25(\mathrm{~s}, 1 \mathrm{H}), 3.72(\mathrm{~s}, 3 \mathrm{H}), 3.68(\mathrm{t}, J$ $=8.5 \mathrm{~Hz}, 1 \mathrm{H}), 2.83(\mathrm{~d}, J=17.2 \mathrm{~Hz}, 1 \mathrm{H}), 2.40-2.31(\mathrm{~m}, 2 \mathrm{H}), 2.29-2.19(\mathrm{~m}, 2 \mathrm{H}), 1.73(\mathrm{~s}, 1 \mathrm{H})$, $1.62-1.47(\mathrm{~m}, 3 \mathrm{H}) ;{ }^{13} \mathbf{C}\left\{{ }^{1} \mathbf{H}\right\}$ NMR (151 MHz, CDCl3) $\delta$ 213.5, 167.2, 164.8, 149.3, 134.0, 
121.3, 72.0, 51.5, 49.5, 35.0, 32.7, 32.1, 23.6; HRMS (ESI) m/z: $[\mathrm{M}+\mathrm{Na}]^{+}$Calcd for $\mathrm{C}_{13} \mathrm{H}_{18} \mathrm{O}_{4} \mathrm{Na}$ 261.1098; Found 261.1097.

Minor diastereomer was unable to be isolated in acceptable purity

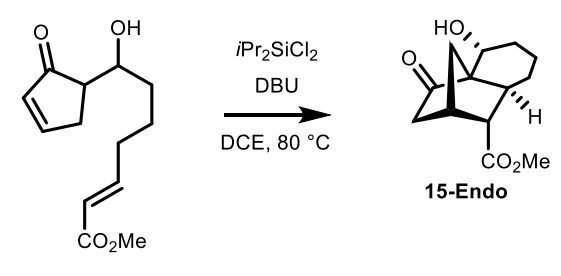

14

Methyl-5-hydroxy-4-oxooctahydro-2H-2,4a-methanonaphthalene-1-carboxylate (15-Endo):

Following a modified general procedure B, $14(0.11 \mathrm{~g}, 0.45 \mathrm{mmol}, 1.0$ equiv, single diastereomer) was reacted with diisopropyldichlorosilane $(0.16 \mathrm{~mL}, 0.91 \mathrm{mmol}, 2.0$ equiv) and DBU $\left(0.41 \mathrm{~mL}, 2.7 \mathrm{mmol}, 6.0\right.$ equiv) in $\mathrm{ClCH}_{2} \mathrm{CH}_{2} \mathrm{Cl}(3.0 \mathrm{~mL})$ from 0 to $80^{\circ} \mathrm{C}$. The product mixture was purified by flash column chromatography using $0-30 \%$ EtOAc in hexanes as the eluting solvent to afford 15-Endo as a white solid (49 $\mathrm{mg} 46 \%$ yield): $\mathbf{R}_{\boldsymbol{f}}=0.29$ (3:7 EtOAc:Hex, Hanessian's stain); ${ }^{1} \mathbf{H}$ NMR $\left(600 \mathrm{MHz}, \mathrm{CDCl}_{3}\right) \delta 5.03(\mathrm{~d}, J=2.3 \mathrm{~Hz}, 1 \mathrm{H}), 4.26(\mathrm{t}$, $J=2.5 \mathrm{~Hz}, 1 \mathrm{H}), 3.68(\mathrm{~s}, 3 \mathrm{H}), 2.90(\mathrm{~s}, 1 \mathrm{H}), 2.63(\mathrm{t}, J=4.3 \mathrm{~Hz}, 1 \mathrm{H}), 2.39(\mathrm{dt}, J=12.0,4.2 \mathrm{~Hz}$, 1H), $2.16(\mathrm{~s}, 2 \mathrm{H}), 2.11(\mathrm{~d}, J=10.4 \mathrm{~Hz}, 1 \mathrm{H}), 2.05-1.97(\mathrm{~m}, 1 \mathrm{H}), 1.85-1.77(\mathrm{~m}, 2 \mathrm{H}), 1.57(\mathrm{~d}, J$ $=10.5 \mathrm{~Hz}, 1 \mathrm{H}), 1.41(\mathrm{dp}, J=13.8,3.6 \mathrm{~Hz}, 1 \mathrm{H}), 1.34(\mathrm{tq}, J=14.2,2.7 \mathrm{~Hz}, 1 \mathrm{H}), 1.20(\mathrm{qd}, J=$ 12.9, $3.8 \mathrm{~Hz}, 1 \mathrm{H}),{ }^{13} \mathbf{C}\left\{{ }^{1} \mathbf{H}\right\}$ NMR $\left(151 \mathrm{MHz}, \mathrm{CDCl}_{3}\right) \delta 221.5,173.6,67.0,59.1,53.4,52.2,42.3$, 38.3, 37.0, 35.3, 31.5, 29.8, 18.6; HRMS (ESI) m/z: $[\mathrm{M}+\mathrm{Na}]^{+}$Calcd for $\mathrm{C}_{13} \mathrm{H}_{18} \mathrm{O}_{4} \mathrm{Na}$ 261.1098; Found 261.1099. The relative stereochemistry of the methine proton at the 6-5 ring junction was established based on its $\sim 4 \mathrm{~Hz}$ coupling constant with the methine proton adjacent 
to the ester. The remaining relative stereochemistry was established by ${ }^{1} \mathrm{H}$ NOESY (see page S94).

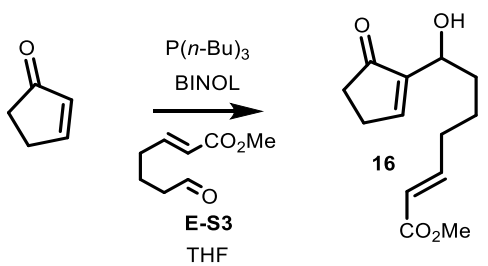

\section{Methyl (E)-7-hydroxy-7-(5-oxocyclopent-1-en-1-yl)hept-2-enoate (16):}

To a round bottom flask charged with THF $(2.0 \mathrm{~mL})$ equipped with a stir bar was added racemic 1,1'-bi-2-naphthol (71 mg, $0.25 \mathrm{mmol}, 0.25$ equiv), 2-cyclopenten-1-one (0.25 mL, $3.0 \mathrm{mmol}$, 3.0 equiv), and E-S3 ( $0.15 \mathrm{~g}, 1.0 \mathrm{mmol}, 1.0$ equiv). The reaction mixture was sparged with argon over 10 minutes, after which tributylphosphine $(0.12 \mathrm{~mL}, 0.50 \mathrm{mmol}, 0.50$ equiv) was added dropwise. The reaction mixture was stirred for 1 hour, after which it was cooled to $0{ }^{\circ} \mathrm{C}$ and the reaction was quenched with $\mathrm{HCl}(1.5 \mathrm{~mL})$. The mixture was transferred to a separatory funnel and the organic layer collected. The aqueous layer was extracted with ethyl acetate $(3 \times 2.0 \mathrm{~mL})$ The crude residue was purified by flash column chromatography using $0-65 \%$ EtOAc in hexanes as the eluting solvent to afford 16 as a clear oil ( $154 \mathrm{mg}, 68 \%$ yield): $\mathbf{R}_{\boldsymbol{f}}=0.42$ (7:3 EtOAc:Hex, $\left.\mathrm{KMnO}_{4}\right) ;{ }^{1} \mathbf{H}$ NMR $\left(600 \mathrm{MHz}, \mathrm{CDCl}_{3}\right) \delta 7.44(\mathrm{~s}, 1 \mathrm{H}), 6.93(\mathrm{dt}, J=16.0,6.5 \mathrm{~Hz}$, 1H), $5.81(\mathrm{~d}, J=15.6 \mathrm{~Hz}, 1 \mathrm{H}), 4.44(\mathrm{~s}, 1 \mathrm{H}), 3.70$ (s, 3H), 2.92 (bs, 1H), 2.61 (s, 2H), $2.49-2.39$ $(\mathrm{m}, 2 \mathrm{H}), 2.23(\mathrm{q}, J=6.8 \mathrm{~Hz}, 2 \mathrm{H}), 1.74-1.59(\mathrm{~m}, 3 \mathrm{H}), 1.58-1.46(\mathrm{~m}, 1 \mathrm{H}) ;{ }^{13} \mathbf{C}\left\{{ }^{1} \mathbf{H}\right\} \mathbf{N M R}(151$ $\left.\mathrm{MHz}, \mathrm{CDCl}_{3}\right) \delta 210.1,167.2,158.1,149.2,147.7,121.4,67.6,51.5,35.4,35.3,32.0,26.7,24.0$; HRMS (ESI) m/z: $[\mathrm{M}+\mathrm{Na}]^{+}$Calcd for $\mathrm{C}_{13} \mathrm{H}_{18} \mathrm{O}_{4} \mathrm{Na} 261.1098$; Found 261.1094. 


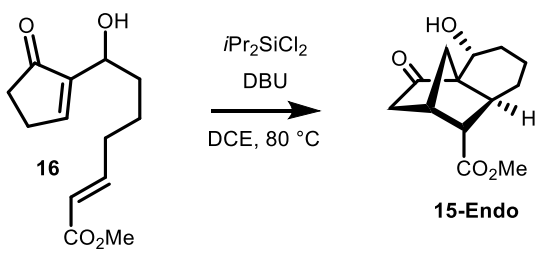

\section{Methyl-5-hydroxy-4-oxooctahydro-2H-2,4a-methanonaphthalene-1-carboxylate (15-Endo):}

Following a modified general procedure B, 16 ( $0.35 \mathrm{~g}, 1.5 \mathrm{mmol}, 1.0$ equiv, single diastereomer $)$ was reacted with diisopropyldichlorosilane $(0.53 \mathrm{~mL}, 2.9 \mathrm{mmol}, 2.0$ equiv) and $\mathrm{DBU}(1.3 \mathrm{~mL}$, $8.8 \mathrm{mmol}, 6.0$ equiv) in $\mathrm{Cl}_{2} \mathrm{CH}_{2} \mathrm{CH}_{2} \mathrm{Cl}(15 \mathrm{~mL})$ from 0 to $80{ }^{\circ} \mathrm{C}$. The product mixture was purified by flash column chromatography using $0-25 \%$ EtOAc in hexanes as the eluting solvent to afford 15-Endo as a white solid (0.13 g 38\% yield). All spectral data match the Diels-Alder adduct of 14 .<smiles>C/C=C/C(=O)O</smiles>

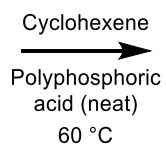<smiles>CC1CC(=O)C2=C1CCCC2</smiles>

\section{3-Methyl-2,3,4,5,6,7-hexahydro-1H-inden-1-one (S7)}

No precaution was made to exclude air or water from the reaction. Following the literature procedure, ${ }^{7}$ crotonic acid (2.6 g, $30 \mathrm{mmol}, 1.0$ equiv) and polyphosphoric acid $(20 \mathrm{~mL})$ were slurried together in a round bottom flask under mechanical stirring due to the viscosity of polyphosphoric acid. This slurry was heated to $60{ }^{\circ} \mathrm{C}$ and cyclohexene was added dropwise. The mixture was mechanically stirred for two hours, after which $10 \%$ aqueous sodium hydroxide (20 $\mathrm{mL}$ ) was added and the mixture was stirred overnight. The crude reaction was transferred to a separatory funnel and diluted with water $(20 \mathrm{~mL})$. This caused a significant exotherm and ice would be recommended instead for dilution. The aqueous mixture was extracted with diethyl 
ether $(3 \times 10 \mathrm{~mL})$. The combined organic layers were washed with sodium bicarbonate $(10 \mathrm{~mL})$, dried over sodium sulfate, and concentrated in vacuo. The crude residue was purified by flash column chromatography using $0-20 \%$ EtOAc in hexanes as the eluting solvent to afford $\mathbf{S} 7$ as a clear oil (0.47 g, 12\% yield): All spectral data are consistent with those reported; ${ }^{7}{ }^{1} \mathbf{H}$ NMR (500 $\left.\mathrm{MHz}, \mathrm{CDCl}_{3}\right) \delta 2.77-2.68(\mathrm{~m}, 1 \mathrm{H}), 2.61(\mathrm{dd}, J=18.5,6.4 \mathrm{~Hz}, 1 \mathrm{H}), 2.40(\mathrm{dt}, J=19.7,6.7 \mathrm{~Hz}$, 1H), $2.21-2.08(\mathrm{~m}, 3 \mathrm{H}), 1.95(\mathrm{dd}, J=18.5,1.9 \mathrm{~Hz}, 1 \mathrm{H}), 1.79-1.63(\mathrm{~m}, 3 \mathrm{H}), 1.63-1.54(\mathrm{~m}$ $1 \mathrm{H}), 1.14(\mathrm{~d}, J=7.2 \mathrm{~Hz}, 3 \mathrm{H})$.
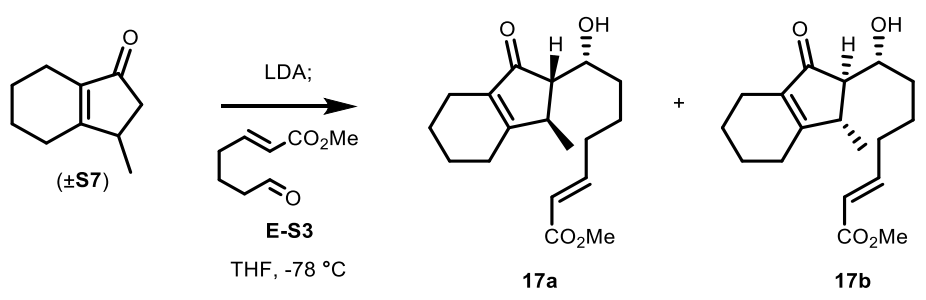

Methyl (E)-7-hydroxy-7-(1-methyl-3-oxo-2,3,4,5,6,7-hexahydro-1H-inden-2-yl)hept-2enoate (17):

Following general procedure A, S7 (0.37 g, $2.7 \mathrm{mmol}, 1.0$ equiv) was deprotonated with LDA (0.32 g, 3.0 mmol, 1.1 equiv) and reacted with E-S3 (0.51 g, $3.3 \mathrm{mmol}, 1.2$ equiv) in THF (7.0 $\mathrm{mL})$ at $-78^{\circ} \mathrm{C}$. The product mixture was purified by flash column chromatography using $0-$ $45 \%$ EtOAc in hexanes as the eluting solvent to afford $\mathbf{1 7} \mathbf{a}$ as a crystalline white solid and $\mathbf{1 7} \mathbf{b}$ as a clear oil (508 mg, 61\% combined yield of 17, 1.3:1 dr):

17a (Major diastereomer):

$\mathbf{R}_{f}=0.53$ (3:2 EtOAc:Hex, UV); ${ }^{1} \mathbf{H}$ NMR $\left(600 \mathrm{MHz}, \mathrm{CDCl}_{3}\right) \delta 6.96(\mathrm{dt}, J=15.6,6.9 \mathrm{~Hz}, 1 \mathrm{H})$, $5.83(\mathrm{~d}, J=15.7 \mathrm{~Hz}, 1 \mathrm{H}), 4.14-4.09(\mathrm{~m}, 1 \mathrm{H}), 3.72(\mathrm{~s}, 3 \mathrm{H}), 2.68(\mathrm{~d}, J=4.9 \mathrm{~Hz}, 1 \mathrm{H}), 2.40(\mathrm{dt}, J$ $=19.0,6.3 \mathrm{~Hz}, 1 \mathrm{H}), 2.30-2.15(\mathrm{~m}, 3 \mathrm{H}), 2.14-2.09(\mathrm{~m}, 2 \mathrm{H}), 2.07(\mathrm{t}, J=2.8 \mathrm{~Hz}, 1 \mathrm{H}), 1.84-$ 
$1.64(\mathrm{~m}, 5 \mathrm{H}), 1.63-1.55(\mathrm{~m}, 1 \mathrm{H}), 1.55-1.47(\mathrm{~m}, 3 \mathrm{H}), 1.18(\mathrm{~d}, J=7.2 \mathrm{~Hz}, 3 \mathrm{H}) ;{ }^{13} \mathbf{C}\left\{{ }^{1} \mathbf{H}\right\} \mathbf{N M R}$ $\left(151 \mathrm{MHz}, \mathrm{CDCl}_{3}\right) \delta 209.2,178.1,167.2,149.2,138.1,121.4,70.6,59.6,51.6,38.0,34.0,32.1$, 26.1, 24.9, 22.3, 21.7, 20.0, 18.4; HRMS (ESI) m/z: $[\mathrm{M}+\mathrm{Na}]^{+}$Calcd for $\mathrm{C}_{18} \mathrm{H}_{26} \mathrm{O}_{4} \mathrm{Na}$ 329.1724; Found 329.1717.

17b (Minor diastereomer):

$\mathbf{R}_{f}=0.63\left(3: 2\right.$ EtOAc:Hex, UV); ${ }^{1} \mathbf{H}$ NMR $\left(600 \mathrm{MHz}, \mathrm{CDCl}_{3}\right) \delta 6.97(\mathrm{dt}, J=15.5,6.9 \mathrm{~Hz}, 1 \mathrm{H})$ $5.83(\mathrm{~d}, J=15.7 \mathrm{~Hz}, 1 \mathrm{H}), 4.56(\mathrm{bs}, 1 \mathrm{H}), 3.71(\mathrm{~s}, 3 \mathrm{H}), 3.62(\mathrm{td}, J=9.2,2.4 \mathrm{~Hz}, 1 \mathrm{H}), 2.40(\mathrm{dt}, J=$ 19.3, $6.3 \mathrm{~Hz}, 1 \mathrm{H}), 2.34(\mathrm{qd}, J=6.6,2.1 \mathrm{~Hz}, 1 \mathrm{H}), 2.30-2.20(\mathrm{~m}, 2 \mathrm{H}), 2.19-2.08(\mathrm{~m}, 3 \mathrm{H}), 1.90$ $(\mathrm{dd}, J=9.5,2.2 \mathrm{~Hz}, 1 \mathrm{H}), 1.80-1.72(\mathrm{~m}, 2 \mathrm{H}), 1.72-1.64(\mathrm{~m}, 2 \mathrm{H}), 1.63-1.49(\mathrm{~m}, 4 \mathrm{H}), 1.18(\mathrm{~d}$, $J=7.2 \mathrm{~Hz}, 3 \mathrm{H}) ;{ }^{13} \mathbf{C}\left\{{ }^{1} \mathbf{H}\right\} \mathbf{N M R}\left(151 \mathrm{MHz}, \mathrm{CDCl}_{3}\right) \delta 211.1,178.0,167.2,149.4,137.4,121.2$, 72.2, 58.2, 51.5, 40.3, 35.2, 32.2, 26.1, 23.7, 22.2, 21.5, 19.9, 18.1; HRMS (ESI) m/z: [M + $\mathrm{Na}]^{+}$Calcd for $\mathrm{C}_{18} \mathrm{H}_{26} \mathrm{O}_{4} \mathrm{Na} 329.1724$; Found 329.1713. For both $\mathbf{1 7 a}$ and $\mathbf{1 7 b}$, the relative stereochemistry of the methyl center and the adjacent methine center was determined by ${ }^{1} \mathrm{H}$ NOESY analysis (see page S101 and S105). The stereochemistry at the alcohol stereocenter was assigned based on analysis of coupling constants (see page S39 for details).
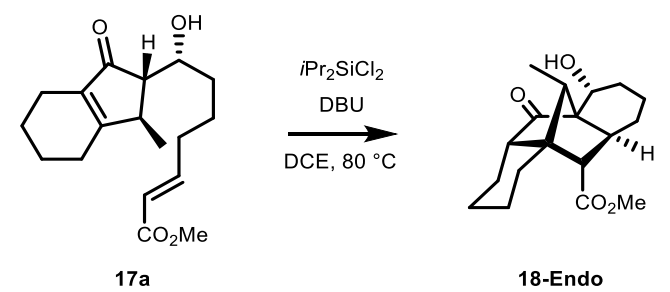

Methyl-4-hydroxy-11-methyl-10-oxododecahydro-4a,8a-methanoanthracene-9-carboxylate (18Endo): 
Following a modified general procedure B, $17 \mathbf{a}(57 \mathrm{mg}, 0.19 \mathrm{mmol}, 1.0$ equiv) was reacted with diisopropyldichlorosilane ( $52 \mu \mathrm{L}, 0.29 \mathrm{mmol}, 1.5$ equiv, single diastereomer) and $\mathrm{DBU}(85 \mu \mathrm{L})$ in $\mathrm{ClCH}_{2} \mathrm{CH}_{2} \mathrm{Cl}(2.0 \mathrm{~mL})$ from 0 to $80^{\circ} \mathrm{C}$. The product mixture was purified by flash column chromatography using $0-25 \%$ EtOAc in hexanes as the eluting solvent to afford 18-Endo as a white crystalline solid (37 mg, 65\% yield): $\mathbf{R}_{f}=0.41$ (3:7 EtOAc:Hex, Hanessian's stain); ${ }^{1} \mathbf{H}$ NMR $\left(600 \mathrm{MHz}, \mathrm{CDCl}_{3}\right) \delta 5.27(\mathrm{~s}, 1 \mathrm{H}), 4.19(\mathrm{~s}, 1 \mathrm{H}), 3.67(\mathrm{~s}, 3 \mathrm{H}), 2.42(\mathrm{dt}, J=11.4,5.7 \mathrm{~Hz}$, $1 \mathrm{H}), 2.31(\mathrm{q}, J=6.9 \mathrm{~Hz}, 1 \mathrm{H}), 2.27(\mathrm{~d}, J=5.0 \mathrm{~Hz}, 1 \mathrm{H}), 2.12(\mathrm{dd}, J=11.7,3.9 \mathrm{~Hz}, 1 \mathrm{H}), 1.94-$ $1.88(\mathrm{~m}, 1 \mathrm{H}), 1.86-1.80(\mathrm{~m}, 3 \mathrm{H}), 1.77(\mathrm{dd}, J=13.9,2.1 \mathrm{~Hz}, 1 \mathrm{H}), 1.74-1.68(\mathrm{~m}, 1 \mathrm{H}), 1.67-$ $1.60(\mathrm{~m}, 2 \mathrm{H}), 1.48-1.35(\mathrm{~m}, 3 \mathrm{H}), 1.31-1.25(\mathrm{~m}, 1 \mathrm{H}), 1.25-1.17(\mathrm{~m}, 1 \mathrm{H}), 1.06(\mathrm{qt}, J=13.2,3.6$ $\mathrm{Hz}, 1 \mathrm{H}), 0.84(\mathrm{~d}, J=6.9 \mathrm{~Hz}, 3 \mathrm{H}) ;{ }^{13} \mathbf{C}\left\{{ }^{1} \mathbf{H}\right\} \mathbf{N M R}\left(151 \mathrm{MHz}, \mathrm{CDCl}_{3}\right) \delta 221.8,175.6,66.0,61.0$, 57.8, 52.6, 52.0(4), 52.0(0), 46.7, 38.5, 31.7, 29.7, 29.0, 25.2, 21.6, 19.8, 18.1, 8.2; HRMS (ESI) $\mathrm{m} / \mathrm{z}:[\mathrm{M}+\mathrm{Na}]^{+}$Calcd for $\mathrm{C}_{18} \mathrm{H}_{26} \mathrm{O}_{4} \mathrm{Na}$ 329.1724; Found 329.1719. Relative stereochemistry was established by ${ }^{1} \mathrm{H}$ NOESY (see page S111). M.P. $109-117^{\circ} \mathrm{C}$.

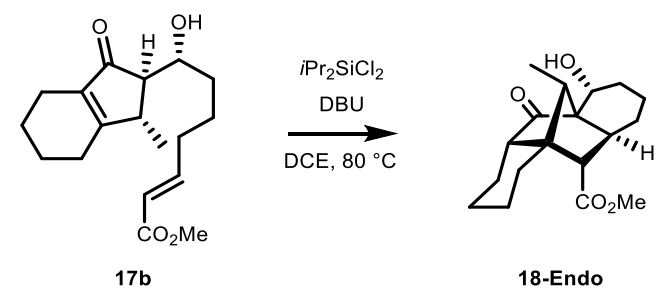

Methyl-4-hydroxy-11-methyl-10-oxododecahydro-4a,8a-methanoanthracene-9-carboxylate (18-

\section{Endo):}

Following a modified general procedure B, $17 \mathbf{b}(0.12 \mathrm{~g})$ was reacted with diisopropyldichlorosilane $(0.10 \mathrm{~mL})$ and $\mathrm{DBU}(0.16 \mathrm{~mL})$ in $\mathrm{Cl}_{2} \mathrm{CH}_{2} \mathrm{CH}_{2} \mathrm{Cl}_{2}(4.0 \mathrm{~mL})$ from 0 to $80^{\circ} \mathrm{C}$. The product mixture was purified by flash column chromatography using $0-20 \%$ EtOAc 
in hexanes as the eluting solvent to afford 18-Endo as a white crystalline solid (54 mg, 45\% yield). All spectral data were identical to the Diels-Alder adduct of $\mathbf{1 7 a}$.

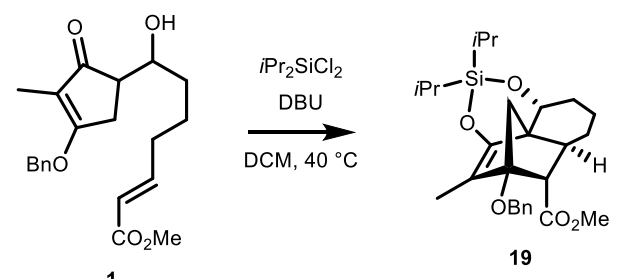

\section{Methyl-5-(benzyloxy)-2,2-diisopropyl-4-methyl-6,6a,7,8,9,9a-hexahydro-5H-3a1,5-} methanonaphtho[1,8-de][1,3,2]dioxasiline-6-carboxylate (19):

Following a modified general procedure B, $1(0.17 \mathrm{~g}, 0.52 \mathrm{mmol}, 1.0$ equiv $)$ was reacted with diisopropyldichlorosilane ( $0.19 \mathrm{~mL}, 1.0 \mathrm{mmol}, 2.0$ equiv) and DBU (0.46 mL, $3.1 \mathrm{mmol}, 6.0$ equiv) in $\mathrm{CH}_{2} \mathrm{Cl}_{2}(5.0 \mathrm{~mL})$ from 0 to $40{ }^{\circ} \mathrm{C}$. In place of the $\mathrm{HF} \cdot$ pyridine workup described in general procedure $\mathrm{B}$, the reaction mixture was instead cooled to $0{ }^{\circ} \mathrm{C}$ and a saturated aqueous solution of sodium bicarbonate $(2 \mathrm{~mL})$ was added slowly. The biphasic mixture was transferred to a separatory funnel and the organic layer collected. The aqueous layer was extracted with $\mathrm{CH}_{2} \mathrm{Cl}_{2}(3 \times 3 \mathrm{~mL})$. The combined organic layers were dried over sodium sulfate and concentrated in vacuo. A neutralized silica gel column was prepared by first passing three column volumes of 5\% triethylamine in hexanes through the packed column. The crude residue was then loaded onto the column as a solution in a minimal amount of toluene and eluted using 0 - 5\% EtOAc:Hex as the eluting solvent to afford 19 as a yellow oil (150 mg, 62\% yield, single diastereomer): $\mathbf{R}_{f}=0.25$ (1:19 EtOAc:Hex, Hanessian's stain); ${ }^{1} \mathbf{H}$ NMR $\left(600 \mathrm{MHz}, \mathrm{CDCl}_{3}\right) \delta$ $7.40(\mathrm{~d}, J=7.5 \mathrm{~Hz}, 2 \mathrm{H}), 7.34$ (t, $J=7.6 \mathrm{~Hz}, 2 \mathrm{H}), 7.28-7.24(\mathrm{~m}, 1 \mathrm{H}), 4.61(\mathrm{~s}, 2 \mathrm{H}), 4.38(\mathrm{~s}, 1 \mathrm{H})$, $3.67(\mathrm{~s}, 3 \mathrm{H}), 2.79(\mathrm{~d}, J=4.0 \mathrm{~Hz}, 1 \mathrm{H}), 2.51-2.44(\mathrm{~m}, 1 \mathrm{H}), 2.13-2.06(\mathrm{~m}, 1 \mathrm{H}), 1.91(\mathrm{~d}, J=7.3$ $\mathrm{Hz}, 1 \mathrm{H}), 1.84(\mathrm{~d}, J=12.7 \mathrm{~Hz}, 1 \mathrm{H}), 1.81-1.72(\mathrm{~m}, 1 \mathrm{H}), 1.58(\mathrm{~s}, 1 \mathrm{H}), 1.51(\mathrm{~s}, 3 \mathrm{H}), 1.47-1.41$ 
$(\mathrm{m}, 3 \mathrm{H}), 1.24-1.18(\mathrm{~m}, 1 \mathrm{H}), 1.12-1.07(\mathrm{~m}, 13 \mathrm{H}) ;{ }^{13} \mathbf{C}\left\{{ }^{1} \mathbf{H}\right\} \mathbf{N M R}\left(151 \mathrm{MHz}, \mathrm{CDCl}_{3}\right) \delta 173.8$, $154.1,139.3,128.4,127.3,127.0,109.9,92.6,70.0,67.7,56.1,51.7,49.8,48.0,41.2,32.1,31.0$, 19.0, 17.2, 16.9(3), 16.9(0), 16.8, 13.6, 12.5, 7.6; HRMS (ESI) m/z: [M + Na $]^{+}$Calcd for $\mathrm{C}_{27} \mathrm{H}_{38} \mathrm{O}_{5} \mathrm{SiNa}$ 493.2381; Found 493.2377. Relative stereochemistry was established in analogy to 2-Endo as well as 21.

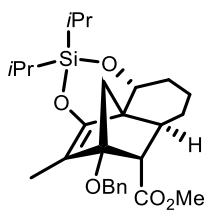

19

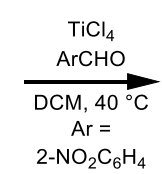

$2-\mathrm{NO}_{2} \mathrm{C}_{6} \mathrm{H}_{4}$

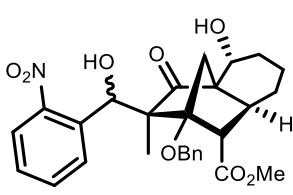

20

Methyl-2-(benzyloxy)-5-hydroxy-3-(hydroxy(2-nitrophenyl)methyl)-3-methyl-4oxooctahydro-2H-2,4a-methanonaphthalene-1-carboxylate (20):

To a round bottom flask equipped with a stir bar was added 19 ( $0.12 \mathrm{~g}, 0.25$ mmol, 1.0 equiv $)$, 2nitro-benzaldehyde (75 mg, $0.50 \mathrm{mmol}, 2.0$ equiv), and $\mathrm{CH}_{2} \mathrm{Cl}_{2}(5.0 \mathrm{~mL})$. Titanium tetrachloride (68 $\mu \mathrm{L}, 0.62 \mathrm{mmol}, 2.5 \mathrm{mmol}$ ) was added dropwise and the reaction mixture was heated to $40{ }^{\circ} \mathrm{C}$ for one hour, after which it was cooled and diluted with $\mathrm{CH}_{2} \mathrm{Cl}_{2}(5 \mathrm{~mL})$. The mixture was transferred to a separatory funnel and washed with a saturated aqueous solution of sodium bicarbonate $(10 \mathrm{~mL})$. The organic layer was collected and the aqueous layer was extracted with $\mathrm{CH}_{2} \mathrm{Cl}_{2}(3 \times 5 \mathrm{~mL})$. The combined organic extracts were washed with brine $(10 \mathrm{~mL})$, dried over sodium sulfate, and concentrated in vacuo. The crude residue was purified by flash column chromatography using $0-50 \%$ EtOAc in hexanes as the eluting solvent to afford $\mathbf{2 0}$ as a foamy white solid. Despite a sizeable $R_{f}$ difference, the two diastereomers failed to separate by either automated or manual flash column chromatography on silica gel. Thus, the product was isolated as a mixture of diastereomers (71 $\mathrm{mg}, 55 \%$ yield of $\mathbf{2 0}, 1.7: 1 \mathrm{dr}): \mathbf{R}_{\boldsymbol{f}}=0.34$ (more polar 
diastereomer) and 0.58 (less polar diastereomer) (3:2 EtOAc:Hex, UV); ${ }^{1} \mathbf{H}$ NMR (600 MHz, $\left.\mathrm{CDCl}_{3}\right) \delta 8.01(\mathrm{~d}, J=8.2 \mathrm{~Hz}, 0.35 \mathrm{H}), 7.70(\mathrm{~d}, J=8.1 \mathrm{~Hz}, 0.59 \mathrm{H}), 7.62-7.56(\mathrm{~m}, 1 \mathrm{H}), 7.54(\mathrm{t}, J$ $=8.0 \mathrm{~Hz}, 1 \mathrm{H}), 7.45-7.40(\mathrm{~m}, 3 \mathrm{H}), 7.40-7.32(\mathrm{~m}, 3 \mathrm{H}), 5.62(\mathrm{bs}, 0.36 \mathrm{H}), 5.35-5.27(\mathrm{~m}, 1 \mathrm{H})$, $5.27-5.22(\mathrm{~m}, 1 \mathrm{H}), 4.57$ (bs, 0.36H), 4.47 (bs, 0.58H), 3.79 (bs, 0.55H), $3.54(\mathrm{~s}, 0.60 \mathrm{H}), 3.44(\mathrm{~s}$, $0.59 \mathrm{H}), 3.30(\mathrm{~s}, 1.1 \mathrm{H}), 3.28(\mathrm{~s}, 1.68 \mathrm{H}), 3.13(\mathrm{dt}, J=12.7,2.6 \mathrm{~Hz}, 0.64 \mathrm{H}), 3.02-2.95(\mathrm{~m}$, $0.76 \mathrm{H}), 2.91(\mathrm{~d}, J=18.1 \mathrm{~Hz}, 0.61 \mathrm{H}), 2.71(\mathrm{dd}, J=10.2,4.3 \mathrm{~Hz}, 0.61 \mathrm{H}), 2.53(\mathrm{dd}, J=9.5,1.5$ $\mathrm{Hz}, 0.36 \mathrm{H}), 2.40(\mathrm{dq}, J=12.9,2.7 \mathrm{~Hz}, 0.37 \mathrm{H}), 2.32(\mathrm{~d}, J=17.9 \mathrm{~Hz}, 0.39 \mathrm{H}), 2.12(\mathrm{~d}, J=19.2$ $\mathrm{Hz}, 0.68 \mathrm{H}), 2.02-1.91(\mathrm{~m}, 1 \mathrm{H}), 1.91-1.85(\mathrm{~m}, 1 \mathrm{H}), 1.83(\mathrm{~s}, 2 \mathrm{H}), 1.76(\mathrm{~d}, J=11.6 \mathrm{~Hz}, 1 \mathrm{H})$, $1.63(\mathrm{~d}, J=13.1 \mathrm{~Hz}, 0.55 \mathrm{H}), 1.51(\mathrm{~s}, 1 \mathrm{H}), 1.45(\mathrm{qd}, J=13.2,3.1 \mathrm{~Hz}, 1 \mathrm{H}), 1.33(\mathrm{t}, J=13.1 \mathrm{~Hz}$, $0.73 \mathrm{H}) ;{ }^{13} \mathbf{C}\left\{{ }^{1} \mathbf{H}\right\} \mathbf{N M R}\left(151 \mathrm{MHz}, \mathrm{CDCl}_{3}\right) \delta 212.2,209.9,185.9,183.5,175.9,173.1,149.2$, 147.1, 139.0, 136.2, 135.5, 135.4, 133.9, 132.9, 129.1, 129.0, 128.9(2), 128.8(6), 128.8, 128.6(4), 128.5(6), 128.1, 127.6, 127.4, 125.3, 124.1, 117.2, 116.0, 73.1, 72.1, 71.8, 71.6, 67.7, 66.5, 56.7, 54.3, 53.7, 52.1, 51.7, 49.7, 36.1, 34.8, 33.7, 33.3, 29.4(4), 29.3(6), 28.8, 25.2, 19.9, 19.5, 6.3, 5.9; HRMS (ESI) m/z: $[\mathrm{M}+\mathrm{Na}]^{+}$Calcd for $\mathrm{C}_{28} \mathrm{H}_{31} \mathrm{NO}_{8} \mathrm{Na}$ 532.1943; Found 532.1925. Relative stereochemistry was established in analogy to $\mathbf{2}$-endo.
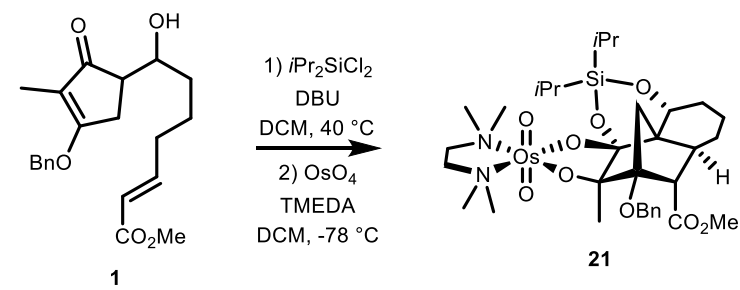

\section{Methyl-5-(benzyloxy)-2,2-diisopropyl-4-methyl-6,6a,7,8,9,9a-hexahydro-5H-3a1,5- methanonaphtho[1,8-de][1,3,2]dioxasiline-6-carboxylate osmate ester (21):}

Following a modified general procedure B, 1 (30 mg, $84 \mu \mathrm{mol}, 1.0$ equiv) was reacted with diisopropyldichlorosilane (30 $\mu \mathrm{L}, 0.17 \mathrm{mmol}, 2.0$ equiv) and DBU (75 $\mu \mathrm{L}, 0.50 \mathrm{mmol}, 6.0$ 
equiv) in $\mathrm{CH}_{2} \mathrm{Cl}_{2}(1.0 \mathrm{~mL})$ from 0 to $40{ }^{\circ} \mathrm{C}$. In place of the $\mathrm{HF} \cdot$ pyridine workup described in general procedure $\mathrm{B}$, the reaction mixture was instead cooled to $0{ }^{\circ} \mathrm{C}$ and a saturated aqueous solution of sodium bicarbonate $(1 \mathrm{~mL})$ was added slowly. The biphasic mixture was transferred to a separatory funnel and the organic layer collected. The aqueous layer was extracted with $\mathrm{CH}_{2} \mathrm{Cl}_{2}(3 \times 3 \mathrm{~mL})$. The combined organic layers were dried over sodium sulfate and concentrated in vacuo. Without further purification, the crude residue was added to a dram vial equipped with a stir bar along with $N, N, N^{\prime}, N^{\prime}$-tetramethyl-ethylenediamine (13 $\mu \mathrm{L}, 92 \mu \mathrm{mol}, 1.1$ equiv) and the mixture was dissolved in 3:1 $\mathrm{Et}_{2} \mathrm{O}: \mathrm{CH}_{2} \mathrm{Cl}_{2}(0.40 \mathrm{~mL})$. The reaction vessel was cooled to $-78{ }^{\circ} \mathrm{C}$ and a solution of osmium tetraoxide in $3: 1 \mathrm{Et}_{2} \mathrm{O}: \mathrm{CH}_{2} \mathrm{Cl}_{2}(0.20 \mathrm{~mL}, 0.42 \underline{\mathrm{M}})$ was added dropwise. The reaction mixture was stirred for 30 minutes, after which it was warmed to room temperature. The crude mixture was passed through a $0.45 \mu \mathrm{m}$ nylon filter and collected in a clean, dry dram vial. A vapor-diffusion crystallization was performed by placing this dram vial, without its cap, into a scintillation vial filled with $5 \mathrm{~mL}$ of $n$-pentane and sealing the scintillation vial. After 24 hours, this afforded $\mathbf{2 1}$ as dark brown crystals (No yield, single diastereomer): $\mathbf{R}_{\boldsymbol{f}}=$ $0.27\left(1: 20 \mathrm{MeOH} / \mathrm{CH}_{2} \mathrm{Cl}_{2}, \mathrm{UV}\right) ;{ }^{1} \mathbf{H}$ NMR $\left(600 \mathrm{MHz}, \mathrm{CDCl}_{3}\right) \delta 7.43(\mathrm{~d}, J=7.4 \mathrm{~Hz}, 2 \mathrm{H}), 7.26$ $(\mathrm{t}, J=7.9 \mathrm{~Hz}, 2 \mathrm{H}), 7.18(\mathrm{t}, J=7.3 \mathrm{~Hz}, 1 \mathrm{H}), 4.93(\mathrm{~d}, J=12.8 \mathrm{~Hz}, 1 \mathrm{H}), 4.76-4.72(\mathrm{~m}, 2 \mathrm{H}), 3.73$ (s, 3H), $3.21(\mathrm{pd}, J=12.1,1.1 \mathrm{~Hz}, 2 \mathrm{H}), 2.93(\mathrm{~s}, 3 \mathrm{H}), 2.92-2.88(\mathrm{~m}, 2 \mathrm{H}), 2.87-2.83(\mathrm{~m}, 1 \mathrm{H})$, $2.78(\mathrm{~s}, 3 \mathrm{H}), 2.73(\mathrm{~s}, 3 \mathrm{H}), 2.56(\mathrm{dd}, J=9.6,1.9 \mathrm{~Hz}, 1 \mathrm{H}), 2.54(\mathrm{~s}, 3 \mathrm{H}), 2.50(\mathrm{~d}, J=5.9 \mathrm{~Hz}, 1 \mathrm{H})$, $2.13-2.07(\mathrm{~m}, 1 \mathrm{H}), 1.89(\mathrm{qt}, J=13.2,3.0 \mathrm{~Hz}, 1 \mathrm{H}), 1.81(\mathrm{~d}, J=12.0 \mathrm{~Hz}, 1 \mathrm{H}), 1.73(\mathrm{~d}, J=9.5$ $\mathrm{Hz}, 1 \mathrm{H}), 1.39-1.27(\mathrm{~m}, 3 \mathrm{H}), 1.22(\mathrm{~s}, 3 \mathrm{H}), 1.17-1.15(\mathrm{~m}, 6 \mathrm{H}), 1.12-1.09(\mathrm{~m}, 1 \mathrm{H}), 1.05(\mathrm{t}, J=$ $7.1 \mathrm{~Hz}, 7 \mathrm{H}) ;{ }^{13} \mathbf{C}\left\{{ }^{1} \mathbf{H}\right\} \mathbf{N M R}\left(151 \mathrm{MHz}, \mathrm{CDCl}_{3}\right) \delta 174.2,141.2,128.0,126.7,126.6,120.0,98.0$, 91.3, 77.4, 77.0, 70.3, 69.0, 64.5, 64.0, 57.9, 52.8, 52.7, 51.7(4), 51.7(2), 50.7, 49.5, 36.6, 34.9, 33.4, 31.3, 19.2, 18.1, 18.0(0), 17.9(5), 17.8, 16.6, 16.5, 13.6; HRMS (ESI) m/z: $[\mathrm{M}+\mathrm{Na}]^{+}$ 
Calcd for $\mathrm{C}_{33} \mathrm{H}_{54} \mathrm{~N}_{2} \mathrm{O}_{9} \mathrm{OsSiNa} 859.3030\left({ }^{186} \mathrm{Os}\right)$; Found 859.3005 M.P. $210{ }^{\circ} \mathrm{C}$ (decomp.).

Relative stereochemistry was established by X-ray crystallography (see page S122).

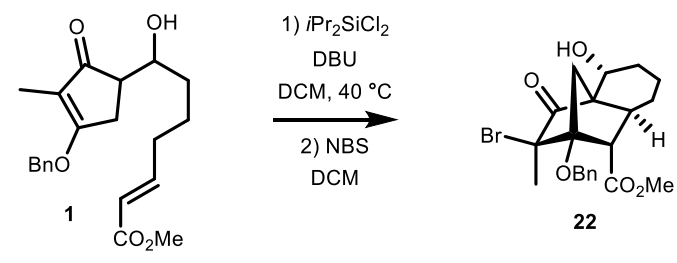

\section{Methyl-2-(benzyloxy)-3-bromo-5-hydroxy-3-methyl-4-oxooctahydro-2H-2,4a- methanonaphthalene-1-carboxylate (22):}

Following a modified general procedure B, 1 (60 mg, $0.17 \mathrm{mmol}, 1.0$ equiv) was reacted with diisopropyldichlorosilane ( $63 \mu \mathrm{L}, 0.34 \mathrm{mmol}, 2.0$ equiv) and $\mathrm{DBU}(0.15 \mathrm{~mL}, 1.0 \mathrm{mmol}, 6.0$ equiv) in $\mathrm{CH}_{2} \mathrm{Cl}_{2}(2.0 \mathrm{~mL})$ from 0 to $40{ }^{\circ} \mathrm{C}$. In place of the $\mathrm{HF} \cdot$ pyridine workup described in general procedure $\mathrm{B}$, the reaction mixture was instead cooled to $0{ }^{\circ} \mathrm{C}$ and a saturated aqueous solution of sodium bicarbonate $(2 \mathrm{~mL})$ was added slowly. The biphasic mixture was transferred to a separatory funnel and the organic layer collected. The aqueous layer was extracted with $\mathrm{CH}_{2} \mathrm{Cl}_{2}(3 \times 3 \mathrm{~mL})$. The combined organic layers were dried over sodium sulfate and concentrated in vacuo. Without further purification, the crude residue was added to a dram vial equipped with a stir bar and dissolved in $\mathrm{CH}_{2} \mathrm{Cl}_{2}(2.0 \mathrm{~mL}) . \mathrm{N}$-Bromo-succinimide $(61 \mathrm{mg}, 0.34$ mmol, 2.0 equiv) was added and the solution was stirred for one hour. After this time, the mixture was transferred to a separatory funnel and washed with a saturated aqueous solution of sodium bicarbonate $(2 \mathrm{~mL})$. The organic layer was collected and the aqueous layer was extracted with $\mathrm{CH}_{2} \mathrm{Cl}_{2}(3 \times 1 \mathrm{~mL})$. The combined organic extracts were dried over sodium sulfate and concentrated in vacuo. The crude residue was purified by flash column chromatography using 0 $-25 \%$ EtOAc in hexanes as the eluting solvent to afford $\mathbf{2 2}$ as a clear oil (38 $\mathrm{mg} 50 \%$ yield over 
two steps, single diastereomer): $\mathbf{R}_{\boldsymbol{f}}=0.25$ (1:4 EtOAc:Hex, Hanessian's stain); ${ }^{1} \mathbf{H}$ NMR (600 $\left.\mathrm{MHz}, \mathrm{CDCl}_{3}\right) \delta 7.47(\mathrm{~d}, J=7.4 \mathrm{~Hz}, 2 \mathrm{H}), 7.38(\mathrm{t}, J=7.4 \mathrm{~Hz}, 2 \mathrm{H}), 7.32(\mathrm{t}, J=7.2 \mathrm{~Hz}, 1 \mathrm{H}), 4.89$ $(\mathrm{d}, J=11.1 \mathrm{~Hz}, 1 \mathrm{H}), 4.75(\mathrm{~s}, 1 \mathrm{H}), 4.70(\mathrm{~d}, J=11.1 \mathrm{~Hz}, 1 \mathrm{H}), 4.33(\mathrm{~s}, 1 \mathrm{H}), 3.71(\mathrm{~s}, 3 \mathrm{H}), 3.05(\mathrm{~d}, J$ $=5.3 \mathrm{~Hz}, 1 \mathrm{H}), 2.62-2.55(\mathrm{~m}, 1 \mathrm{H}), 2.40(\mathrm{~d}, J=10.2 \mathrm{~Hz}, 1 \mathrm{H}), 2.26(\mathrm{~d}, J=10.1 \mathrm{~Hz}, 1 \mathrm{H}), 2.14(\mathrm{~d}$, $J=9.8 \mathrm{~Hz}, 1 \mathrm{H}), 1.92-1.79(\mathrm{~m}, 2 \mathrm{H}), 1.73(\mathrm{~s}, 3 \mathrm{H}), 1.43(\mathrm{~d}, J=13.6 \mathrm{~Hz}, 1 \mathrm{H}), 1.31(\mathrm{t}, J=13.1$ $\mathrm{Hz}, 1 \mathrm{H}), 1.19(\mathrm{qd}, J=13.5,4.0 \mathrm{~Hz}, 1 \mathrm{H}) ;{ }^{13} \mathbf{C}\left\{{ }^{1} \mathbf{H}\right\} \mathbf{N M R}\left(151 \mathrm{MHz}, \mathrm{CDCl}_{3}\right) \delta 213.3,172.1$, $137.9,128.6,128.0,127.4,89.0,68.8,67.0,66.8,56.0,52.4,50.6,39.8,33.7,32.5,29.3,20.5$, 17.9; HRMS (ESI) $\mathrm{m} / \mathrm{z}:[\mathrm{M}+\mathrm{Na}]^{+}$Calcd for $\mathrm{C}_{21} \mathrm{H}_{25} \mathrm{BrO}_{5} \mathrm{Na} 459.0778\left({ }^{79} \mathrm{Br}\right)$ and 461.0761 $\left({ }^{81} \mathrm{Br}\right)$; Found 459.0774 and 461.0768 , respectively. Relative stereochemistry was established in analogy to 2-Endo.

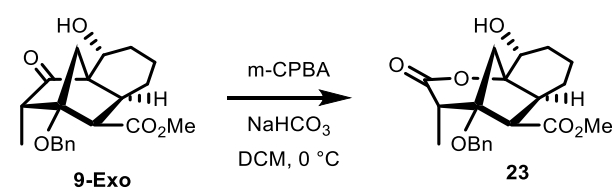

\section{Methyl-4-(benzyloxy)-9-hydroxy-3-methyl-2-oxooctahydro-2H-4,9a methanobenzo[b]oxepine-5-carboxylate (23):}

No precaution was made to exclude air or water from the reaction. To a round bottom flask equipped with a stir bar and charged with $\mathrm{CH}_{2} \mathrm{Cl}_{2}(10 \mathrm{~mL})$ was added 9-Exo $(0.20 \mathrm{~g}, 0.55 \mathrm{mmol}$, 1.0 equiv) and sodium bicarbonate ( $0.18 \mathrm{~g}, 2.2 \mathrm{mmol}, 4.0$ equiv). The mixture was cooled to 0 ${ }^{\circ} \mathrm{C}$ and meta-chloroperbenzoic acid $(0.19 \mathrm{~g}, 1.1 \mathrm{mmol}, 2.0$ equiv) was added. The solution was stirred at $0{ }^{\circ} \mathrm{C}$ for 13 hours, after which a saturated solution of sodium sulfate $(10 \mathrm{~mL})$ was added and the mixture was warmed to ambient temperature. The mixture was transferred to a separatory funnel, the organic layer collected, and the aqueous layer extracted with EtOAc ( $3 \times 5$ $\mathrm{mL})$. The combined organic extracts were dried over sodium thiosulfate and concentrated in 
vacuo. The crude residue was purified by flash column chromatography using $0-45 \%$ EtOAc in hexanes as the eluting solvent to afford $\mathbf{2 3}$ as a foamy white solid $(0.17 \mathrm{~g})$ containing $2.7 \mathrm{wt} \%$ $\mathrm{CH}_{2} \mathrm{Cl}_{2}$ (82\% yield, single regioisomer): $\mathbf{R}_{\boldsymbol{f}}=0.32$ (1:1 EtOAc:Hex, Hanessian's stain); ${ }^{1} \mathbf{H}$ NMR $\left(500 \mathrm{MHz}, \mathrm{CDCl}_{3}\right) \delta 7.36-7.27(\mathrm{~m}, 5 \mathrm{H}), 4.54(\mathrm{~d}, J=11.2 \mathrm{~Hz}, 1 \mathrm{H}), 4.47(\mathrm{~d}, J=11.2 \mathrm{~Hz}$, $1 \mathrm{H}), 4.01(\mathrm{dd}, J=6.1,2.3 \mathrm{~Hz}, 1 \mathrm{H}), 3.62(\mathrm{~s}, 3 \mathrm{H}), 3.32(\mathrm{~d}, J=8.9 \mathrm{~Hz}, 1 \mathrm{H}), 3.10(\mathrm{q}, J=7.0 \mathrm{~Hz}$, 1H), $2.90(\mathrm{~d}, J=12.1 \mathrm{~Hz}, 1 \mathrm{H}), 2.59-2.51(\mathrm{~m}, 1 \mathrm{H}), 2.05(\mathrm{~d}, J=12.3 \mathrm{~Hz}, 1 \mathrm{H}), 1.95-1.79(\mathrm{~m}$, 2H), $1.76-1.66(\mathrm{~m}, 1 \mathrm{H}), 1.63-1.55(\mathrm{~m}, 2 \mathrm{H}), 1.53-1.47(\mathrm{~m}, 1 \mathrm{H}), 1.37(\mathrm{~d}, J=7.1 \mathrm{~Hz}, 3 \mathrm{H})$; ${ }^{13} \mathbf{C}\left\{{ }^{1} \mathbf{H}\right\}$ NMR $\left(126 \mathrm{MHz}, \mathrm{CDCl}_{3}\right) \delta 172.6,169.7,137.9,128.5,127.7,127.0,89.5,82.6,69.1$, 66.7, 51.6, 49.8, 47.0, 44.5, 39.1, 27.6, 25.5, 18.1, 9.7; HRMS (ESI) m/z: $[\mathrm{M}+\mathrm{Na}]^{+}$Calcd for $\mathrm{C}_{21} \mathrm{H}_{26} \mathrm{O}_{6} \mathrm{Na}$ 397.1622; Found 397.1615. 


\section{Unsuccessful substrates}

We discovered a number of substrates that were not compatible with our reaction conditions in the course of our investigation (Table S1). In an attempt to expand our methodology to 6-membered dienes, we synthesized substrate S8. However, after being subjected to our standard reaction conditions, only starting material was recovered. This method is also ineffective for substrates that would pass through a particularly high activation barrier in the course of the IMDA reaction. For example, the desired IMDA reaction with S9, which would form a strained 67 ring system, was out-competed by elimination of the alcohol. This same outcome was observed for $\mathbf{S 1 1}$, which lacks significant activation for either the diene or the dienophile.

\section{Table S1. Unsuccessful substrates ${ }^{a}$}

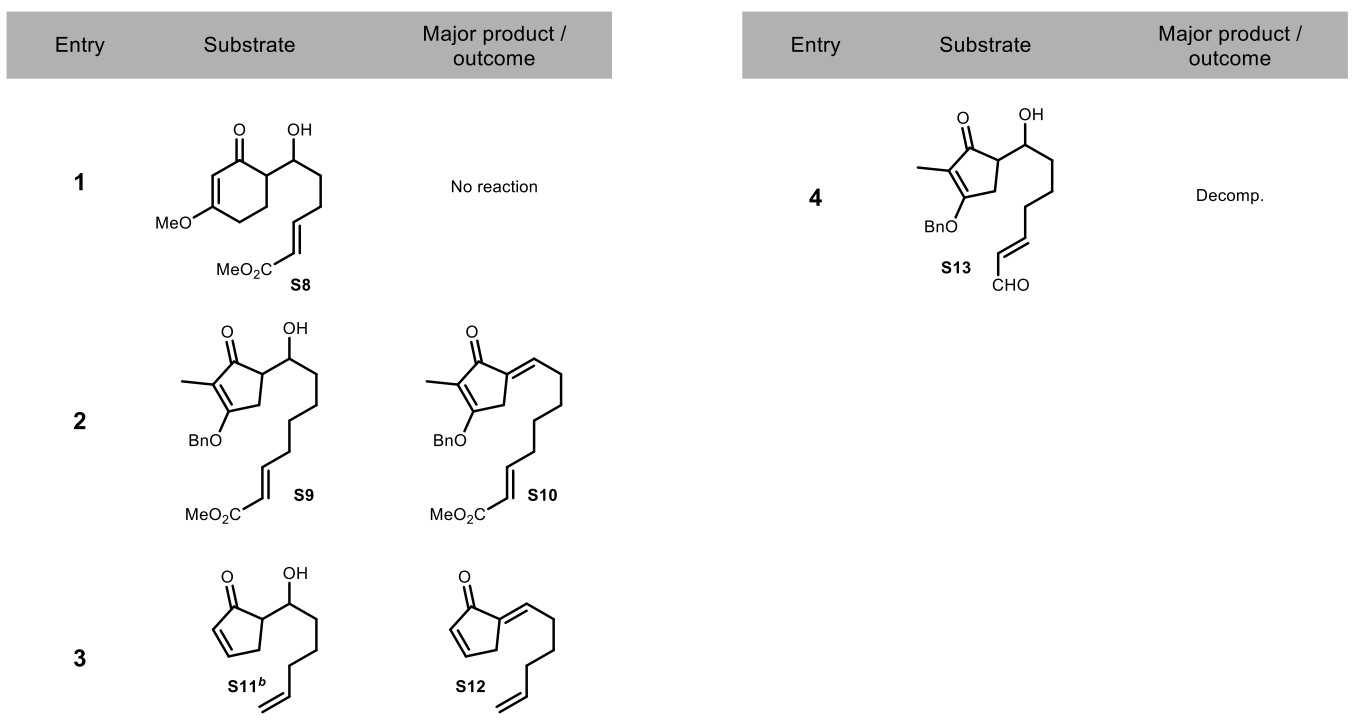

${ }^{a}$ Standard conditions: $i \mathrm{Pr}_{2} \mathrm{SiCl}_{2}$ (2 equiv), $\mathrm{DBU}$ (6 equiv), dry $\mathrm{CH}_{2} \mathrm{Cl}_{2}, 0-40{ }^{\circ} \mathrm{C}, 16$ h. ${ }^{b}$ Reaction was run in $\mathrm{dry}_{2} \mathrm{Cl}_{2} \mathrm{CH}_{2} \mathrm{CH}_{2} \mathrm{Cl}_{2}$ from $0-80^{\circ} \mathrm{C}$

Finally, certain functional groups are not well tolerated under our conditions. With an enal as the dienophile, S13 yielded only a complex mixture after an attempted IMDA cyclization. If the reactive diene is derived from a vinylogous amide, as in the case of S14, a small amount of the desired product $\mathbf{S 1 5}$ is 
isolated, but the major product is $\mathbf{S 1 6}$, which may arise directly from $\mathbf{S 1 4}$ via a Michael addition or from the initial Diels-Alder adduct via a retro Michael addition. 


\section{Stereochemical assignment of $17 \mathrm{a}$ and $17 \mathrm{~b}$}

For compounds $\mathbf{1 7} \mathbf{a}$ and $\mathbf{1 7 b}$, the relative stereochemistry of the two stereocenters within the cyclopentenone ring were established by ${ }^{1} \mathrm{H}$ NOSEY. However, these NOESY experiments did not conclusively assign the relative configuration of the alcohol stereocenter. Using a similar line of reasoning to that established by Dogan and Erol, ${ }^{8}$ we hypothesized that this stereocenter could be assigned through an analysis of coupling constants based on a rigidifying hydrogen bonding interaction between the alcohol and the ketone (Figure S1). Such an interaction would lead to a dihedral angle of approximately $60^{\circ}$ between $\mathrm{H}_{\mathrm{a}}$ and $\mathrm{H}_{\mathrm{b}}$ for the syn aldol adduct and $180^{\circ}$ for the anti aldol adduct. Based on the Karplus equation, these angles would yield ${ }^{3} \mathrm{~J}_{\mathrm{Ha}-\mathrm{Hb}}$ coupling constants of $5 \mathrm{~Hz}$ and $15 \mathrm{~Hz}$ for the syn and anti aldol adducts, respectively.

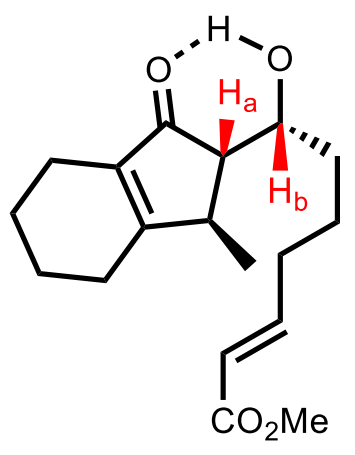

Syn aldol adduct

$$
\begin{gathered}
\varphi_{\mathrm{ab}} \approx 60^{\circ} \\
{ }^{3} J_{\mathrm{Ha}-\mathrm{Hb}} \approx 5 \mathrm{~Hz}
\end{gathered}
$$

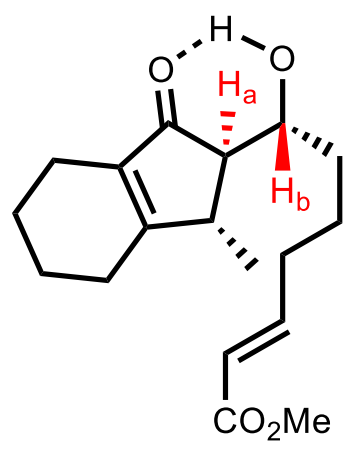

Anti aldol adduct

$$
\begin{gathered}
\varphi_{\mathrm{ab}} \approx 180^{\circ} \\
{ }^{3} \mathrm{~J}_{\mathrm{Ha}-\mathrm{Hb}} \approx 15 \mathrm{~Hz}
\end{gathered}
$$

Figure S1. Predicted dihedral angles between $\mathrm{H}_{a}$ and $\mathrm{H}_{\mathrm{b}}$ for compounds $\mathbf{1 7} \mathbf{a}$ and $\mathbf{1 7 b}$ based on a rigidifying hydrogen bonding interaction between the alcohol and ketone.

In order to test the legitimacy of our hypothetical hydrogen bonding interaction in Figure S1, we calculated the lowest energy conformers for both the syn and anti aldol adducts. A conformer search was carried out in Spartan using the MMFF force field. A sample of 8-12 
structures were carried into dft calculations, and the lowest energy conformer for each isomer is shown in Figure S2. The dft calculations used $\omega$ B97X-D/6-31G* method and basis set. The calculated dihedral angles between $\mathrm{H}_{\mathrm{a}}$ and $\mathrm{H}_{\mathrm{b}}$ are in good agreement with our hypothesis in Figure S1.

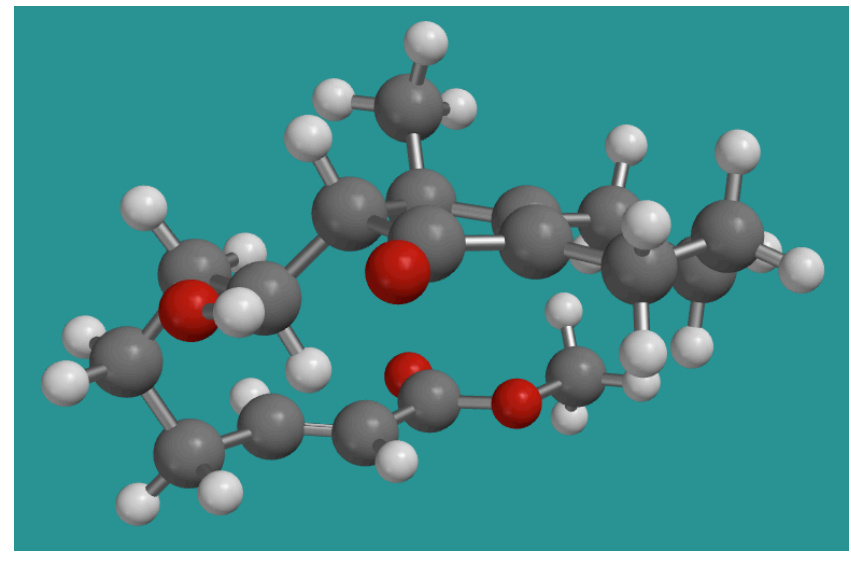

anti aldol

$\varphi_{a b}=-176.6^{\circ}$

E: -1002.187660 hartrees

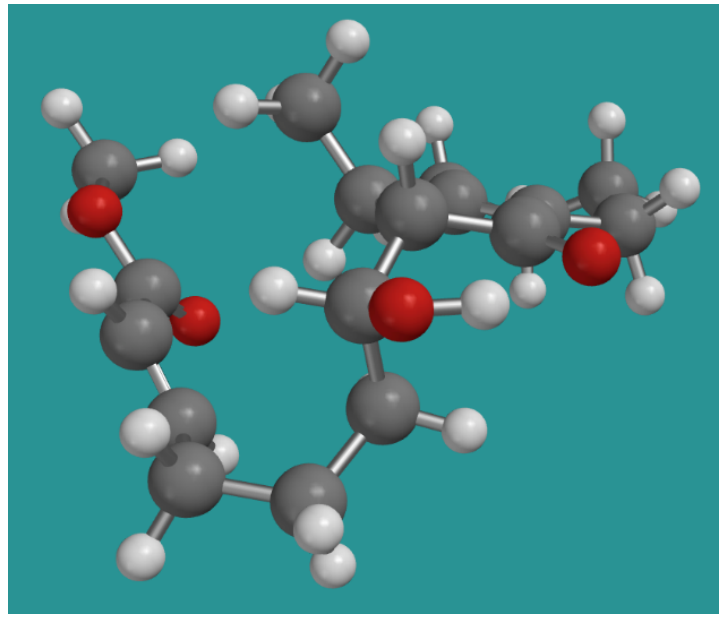

syn aldol

$\varphi_{a b}=66.1^{\circ}$

E: -1002.186720 hartrees

Figure S2. Calculated lowest energy conformations for the syn and anti aldol adducts of $\mathbf{1 7}$. Calculated dihedral angles between $\mathrm{H}_{a}$ and $\mathrm{H}_{b}$ are reported.

As the calculated lowest energy conformers for both the syn and anti aldol adducts were in agreement with our hypothesis in Figure 1, we assigned 17a as the syn aldol adduct based on an observed ${ }^{3} \mathrm{~J}_{\mathrm{Ha}-\mathrm{Hb}}$ of $\sim 2.5 \mathrm{~Hz}$. Similarly, 17b was assigned as the anti aldol adduct based on an observed ${ }^{3} \mathrm{~J}_{\mathrm{Ha}-\mathrm{Hb}}$ of $9.5 \mathrm{~Hz}$. 


\section{References}

(1) Marshall, J. A.; Piettre, A.; Paige, M. A.; Valeriote, F. A Modular Synthesis of Annonaceous Acetogenins. J. Org. Chem. 2003, 68 (5), 1771-1779.

(2) Daniel Little, R.; Fox, D. P.; Van Hijfte, L.; Dannecker, R.; Sowell, G.; Wolin, R. L.; Moëns, L.; Baizer, M. M. Electroreductive Cyclization. Ketones and Aldehydes Tethered to $\alpha, \beta$-Unsaturated Esters (Nitriles). Fundamental Investigations. J. Org. Chem. 1988, 53, $2287-2294$.

(3) Burns, A. S.; Rychnovsky, S. D. Total Synthesis and Structure Revision of (-)-Illisimonin A, a Neuroprotective Sesquiterpenoid from the Fruits of Illicium Simonsii. J. Am. Chem. Soc. 2019, 141, 13295-13300.

(4) Bull, J. A.; Charette, A. B. Intramolecular Simmons-Smith Cyclopropanation. Studies into the Reactivity of Alkyl-Substituted Zinc Carbenoids, Effect of Directing Groups and Synthesis of Bicyclo[n.1.0]Alkanes. J. Am. Chem. Soc. 2010, 132, 1895-1902.

(5) An, Q.; Shen, J.; Butt, N.; Liu, D.; Liu, Y.; Zhang, W. Asymmetric Domino Double Michael Addition of Nitroolefins and Aldehyde Esters with Trans-Perhydroindolic Acid as an Organocatalyst. Synth. 2013, 45, 1612-1623.

(6) Maynard, H. D.; Grubbs, R. H. Purification Technique for the Removal of Ruthenium from Olefin Metathesis Reaction Products. Tetrahedron Lett. 1999, 40, 4137-4140.

(7) Batsanov, A. S.; Bridgewater, B. M.; Howard, J. A. K.; Hughes, A. K.; Wilson, C. Sterically Demanding Cyclopentadienyl Chemistry: Synthesis of Iron and Zirconium Complexes of 1-Phenyl-3-Methyl-4,5,6,7-Tetrahydroindenyl. J. Organomet. Chem. 1999, 
590, 169-179.

(8) Erol, S.; Dogan, I. Stereochemical Assignments of Aldol Products of 2-Arylimino-3-ArylThiazolidine-4-Ones by ${ }^{1}$ H NMR. Magn. Reson. Chem. 2012, 50, 402-405. 


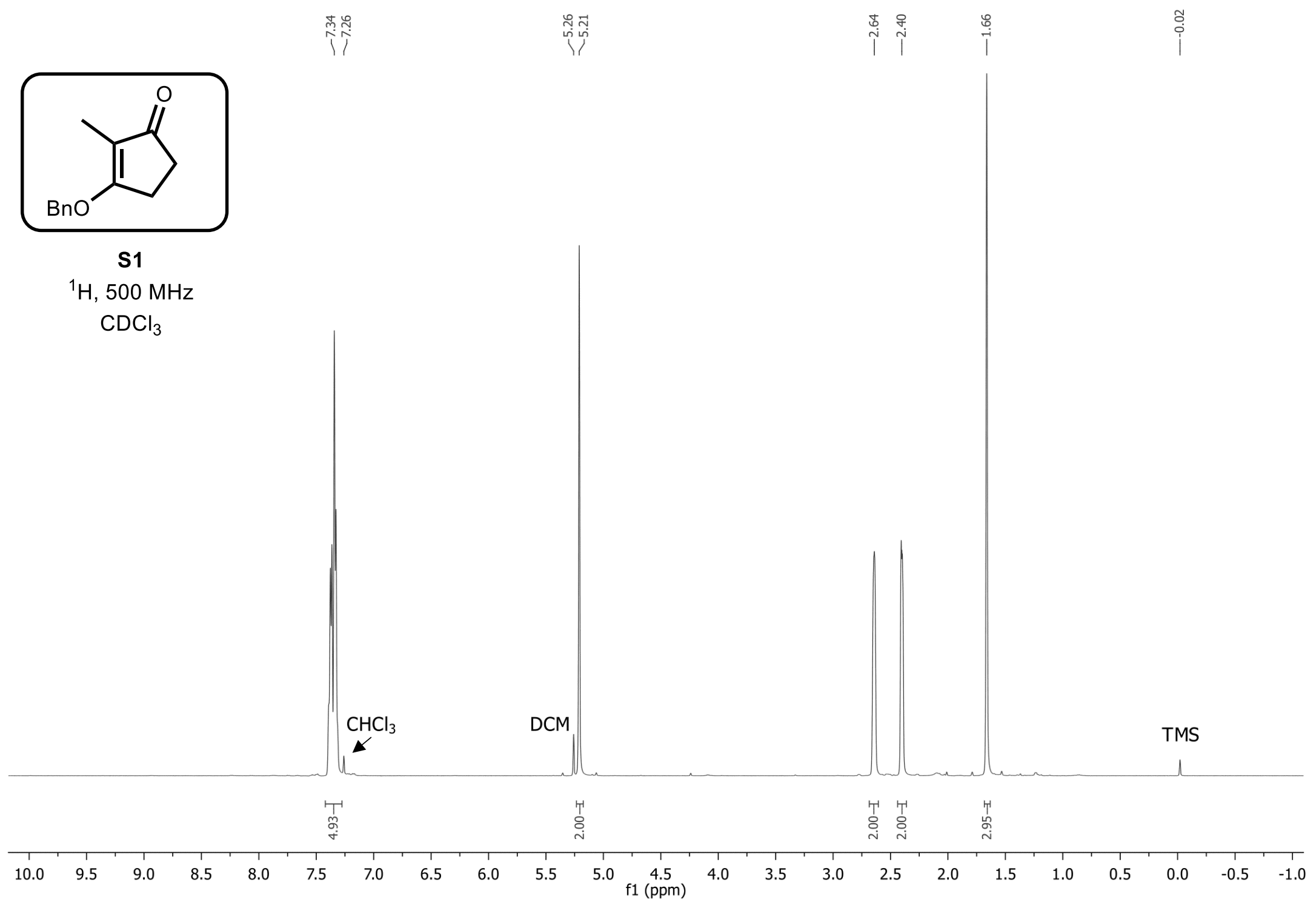




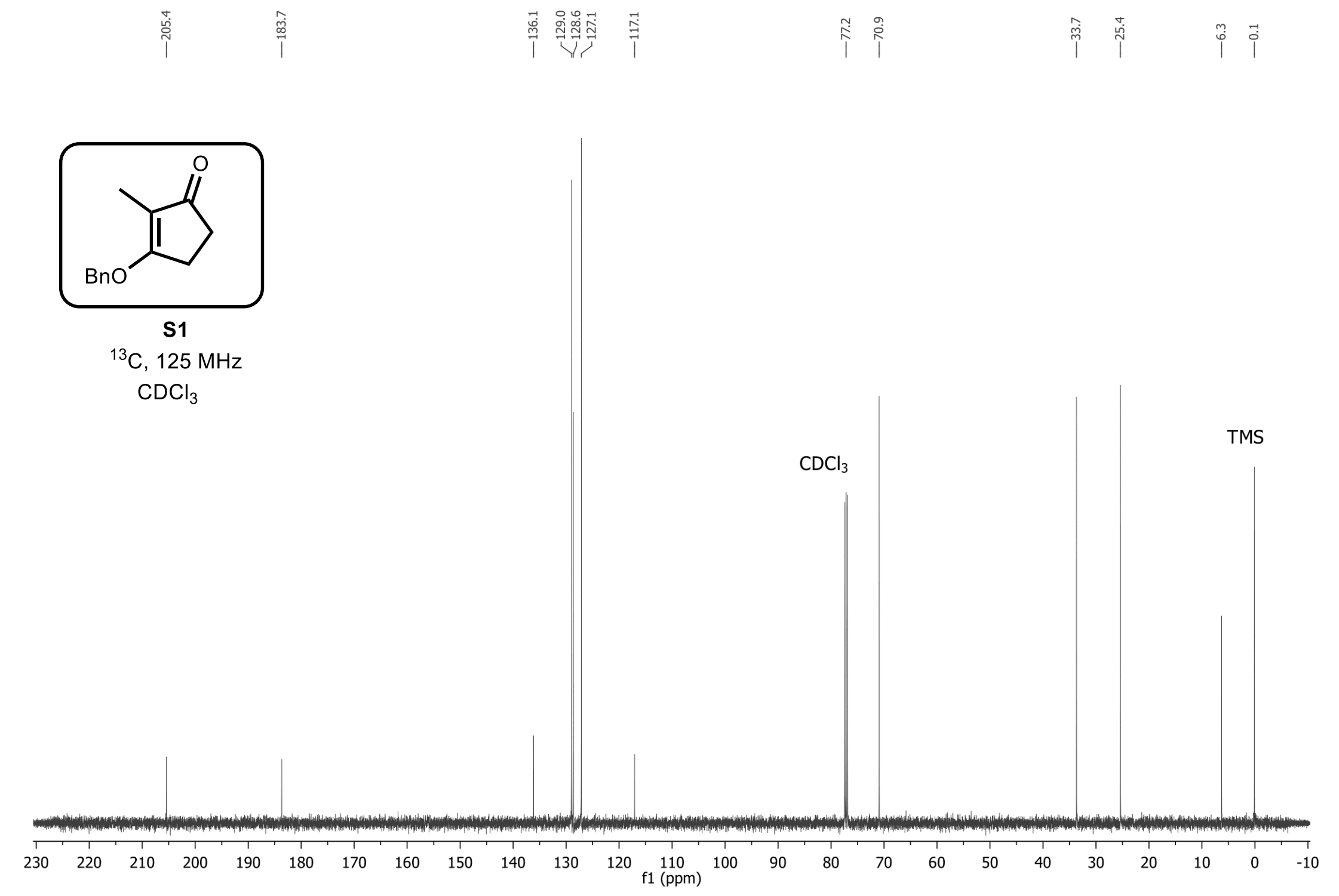




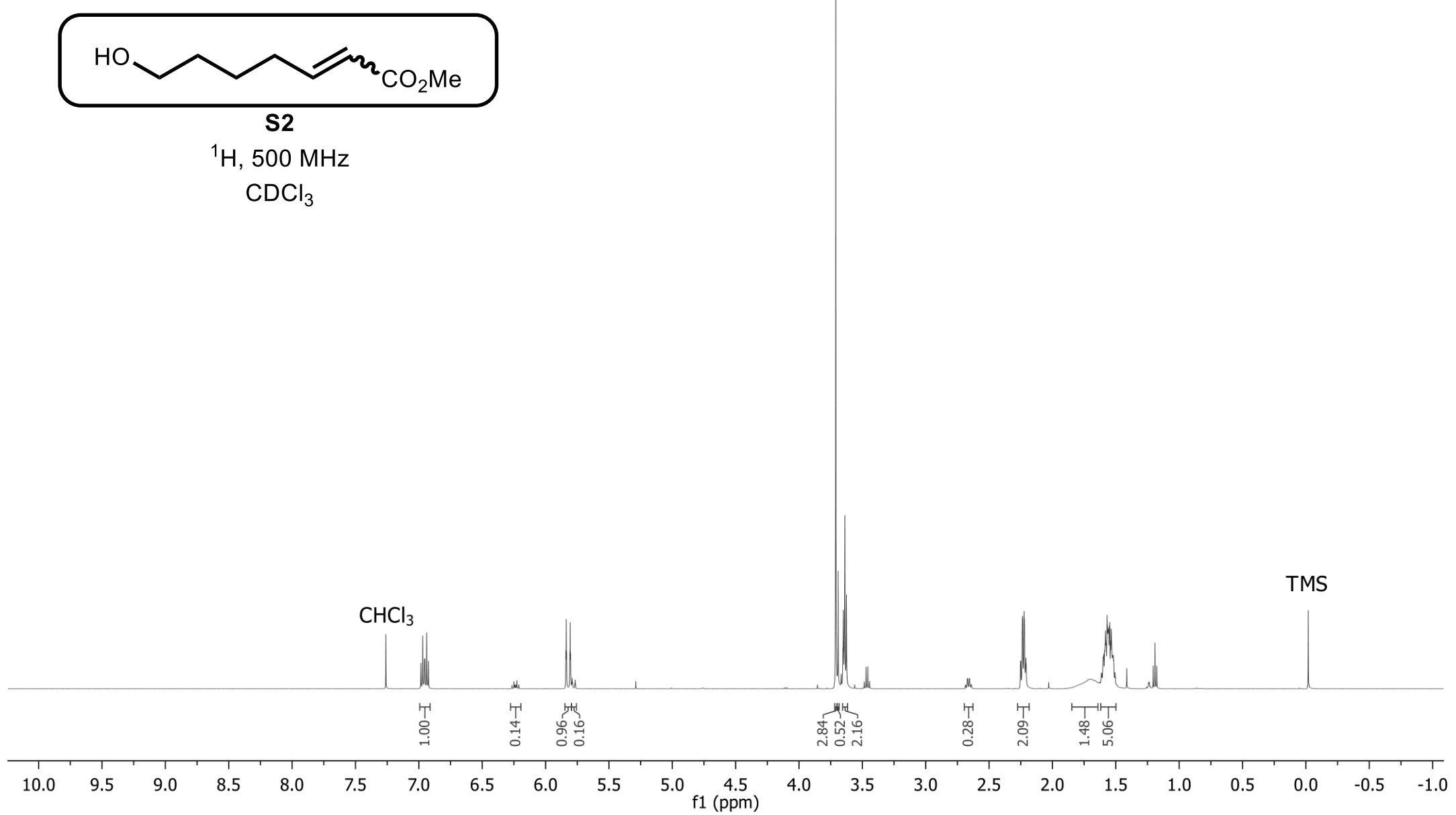




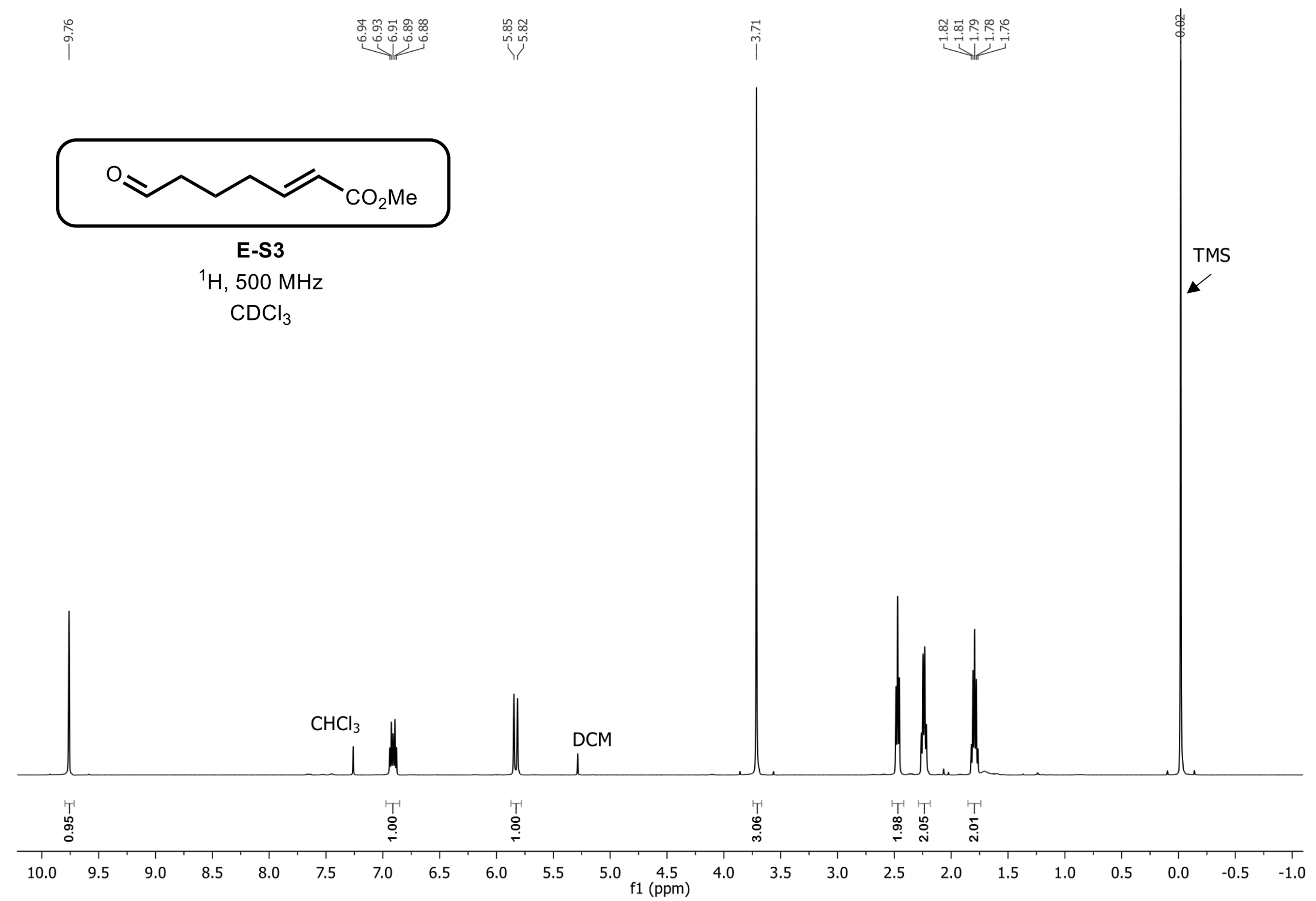




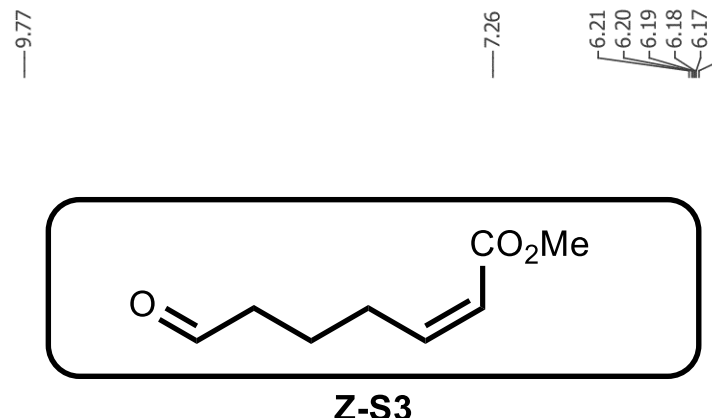

, $500 \mathrm{MHz}$

$\mathrm{CDCl}_{3}$

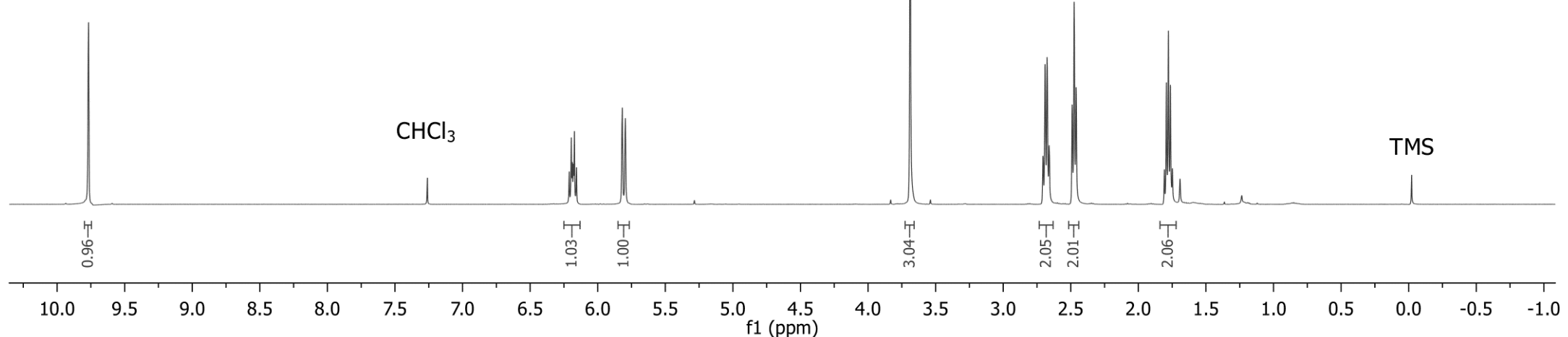




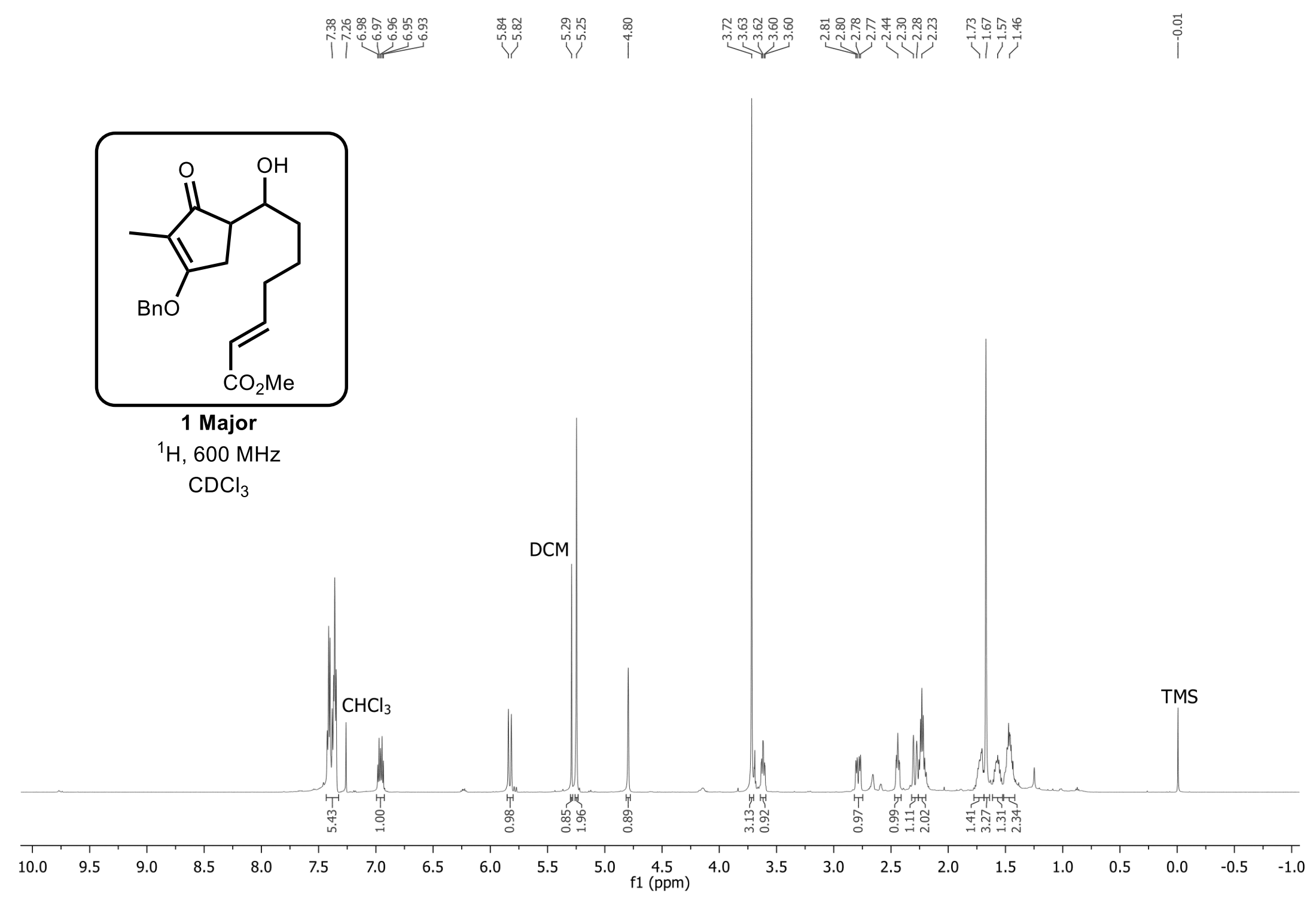




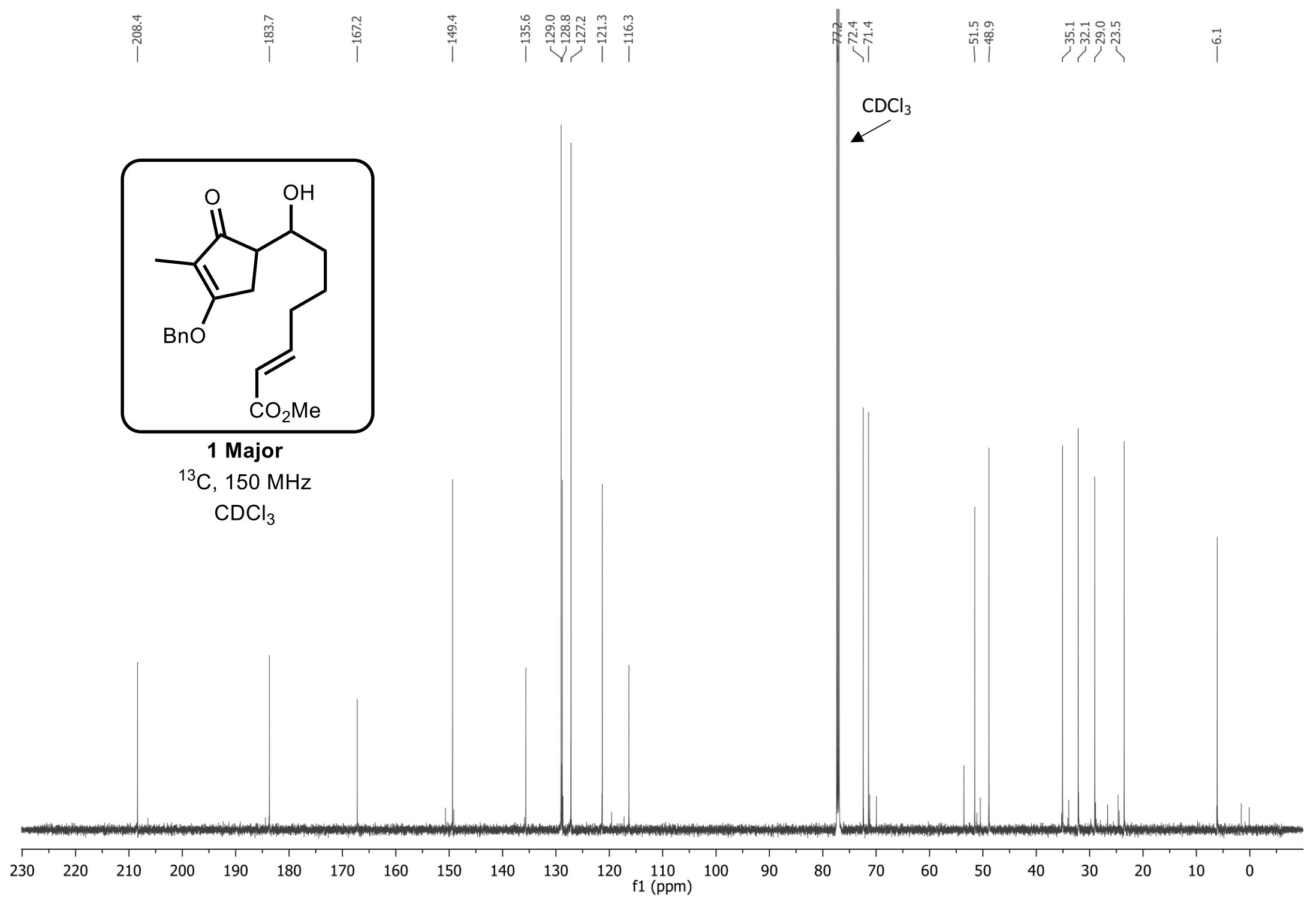




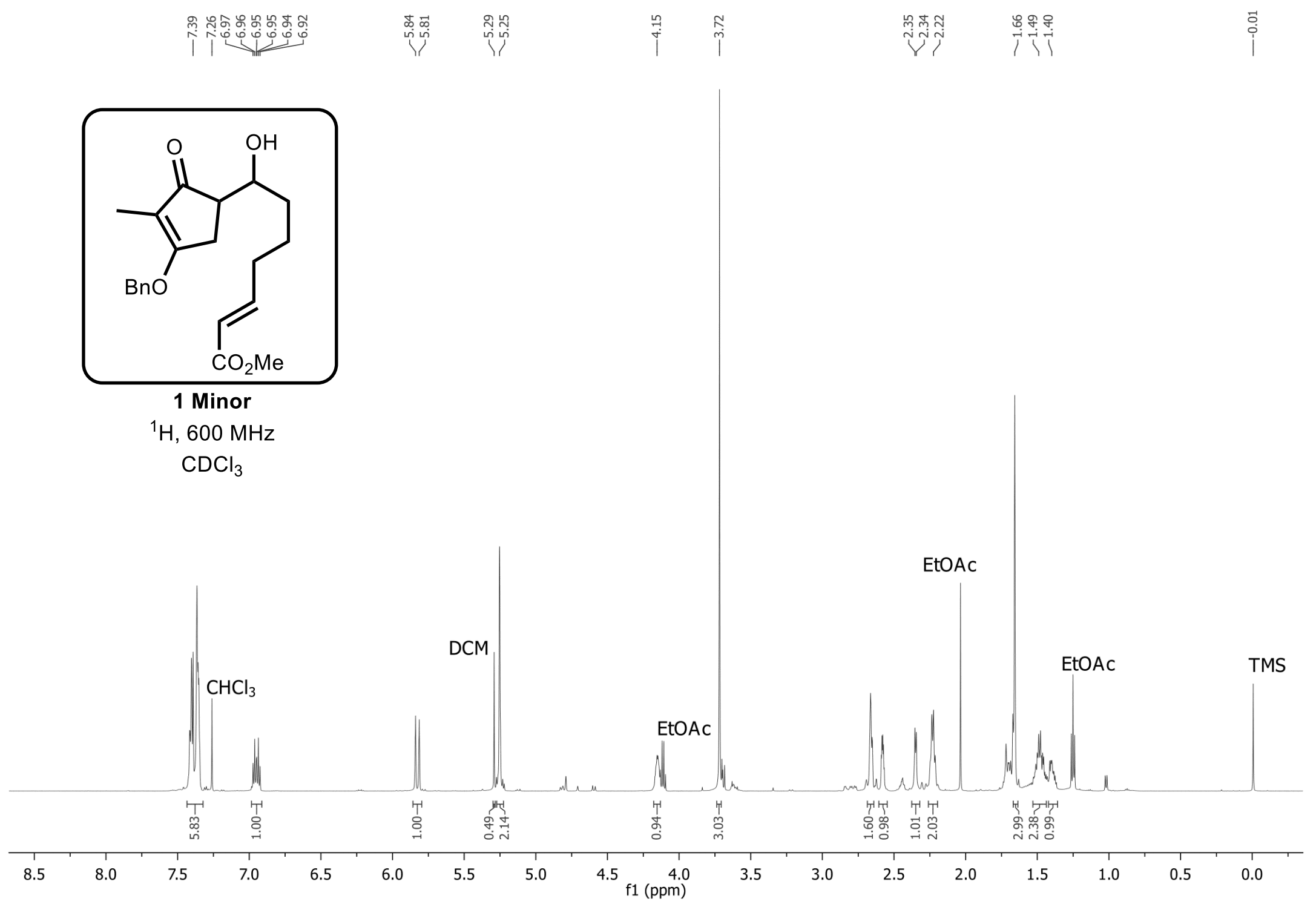




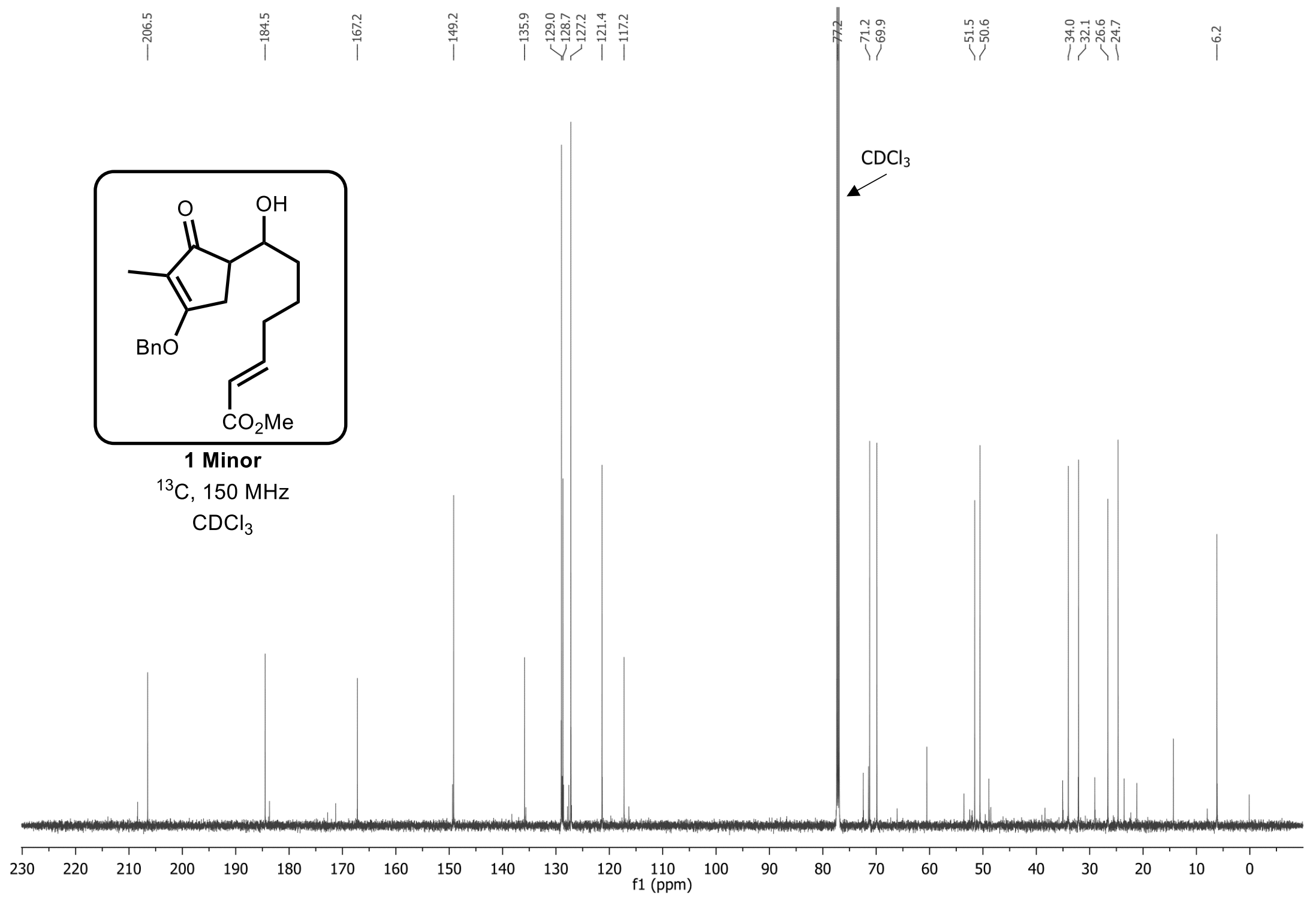




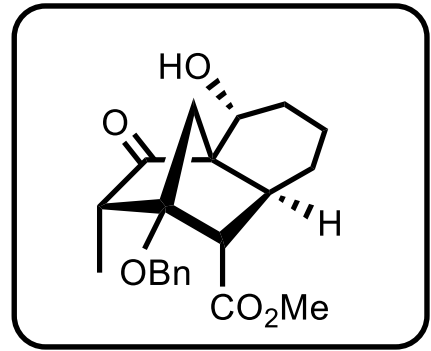

2-Endo

${ }^{1} \mathrm{H}, 600 \mathrm{MHz}$ $\mathrm{CDCl}_{3}$

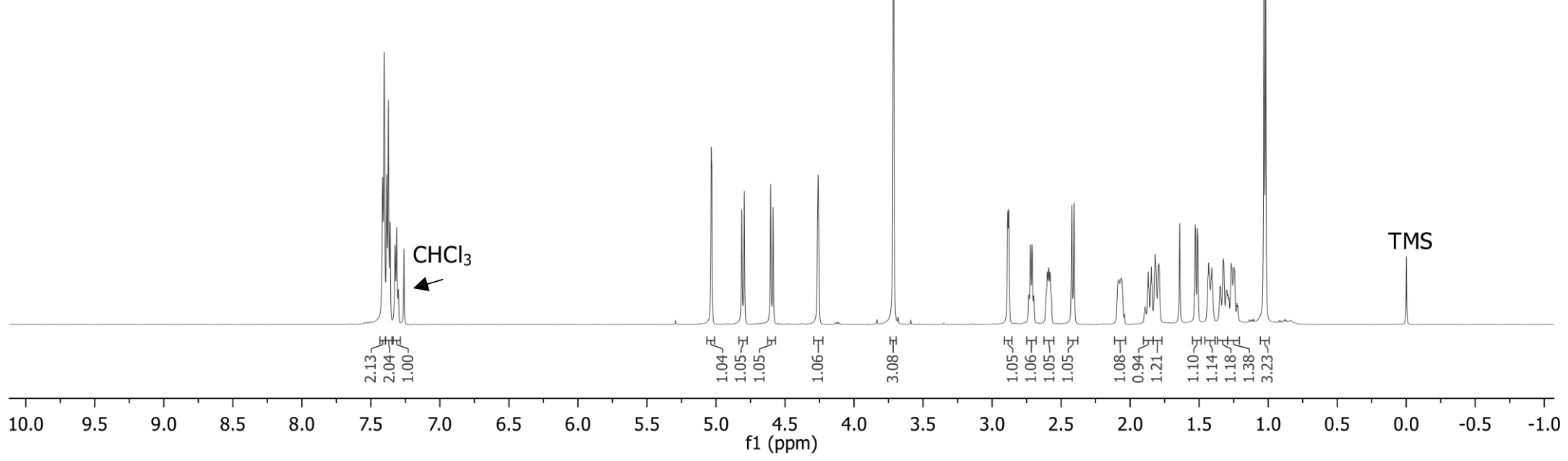




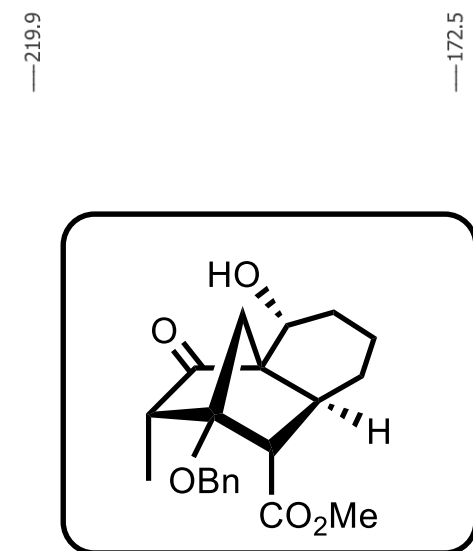

2-Endo

${ }^{13} \mathrm{C}, 150 \mathrm{MHz}$

$\mathrm{CDCl}_{3}$

$\mathrm{CDCl}_{3}$

$\leftarrow$

\begin{tabular}{lllllllllllll}
\hline 30 & 220 & 210 & 200 & 190 & 180 & 170 & 160 & 150 & 140 & 130 & 120 & $\underset{f 1}{110}(\mathrm{ppm})$ \\
100
\end{tabular} 


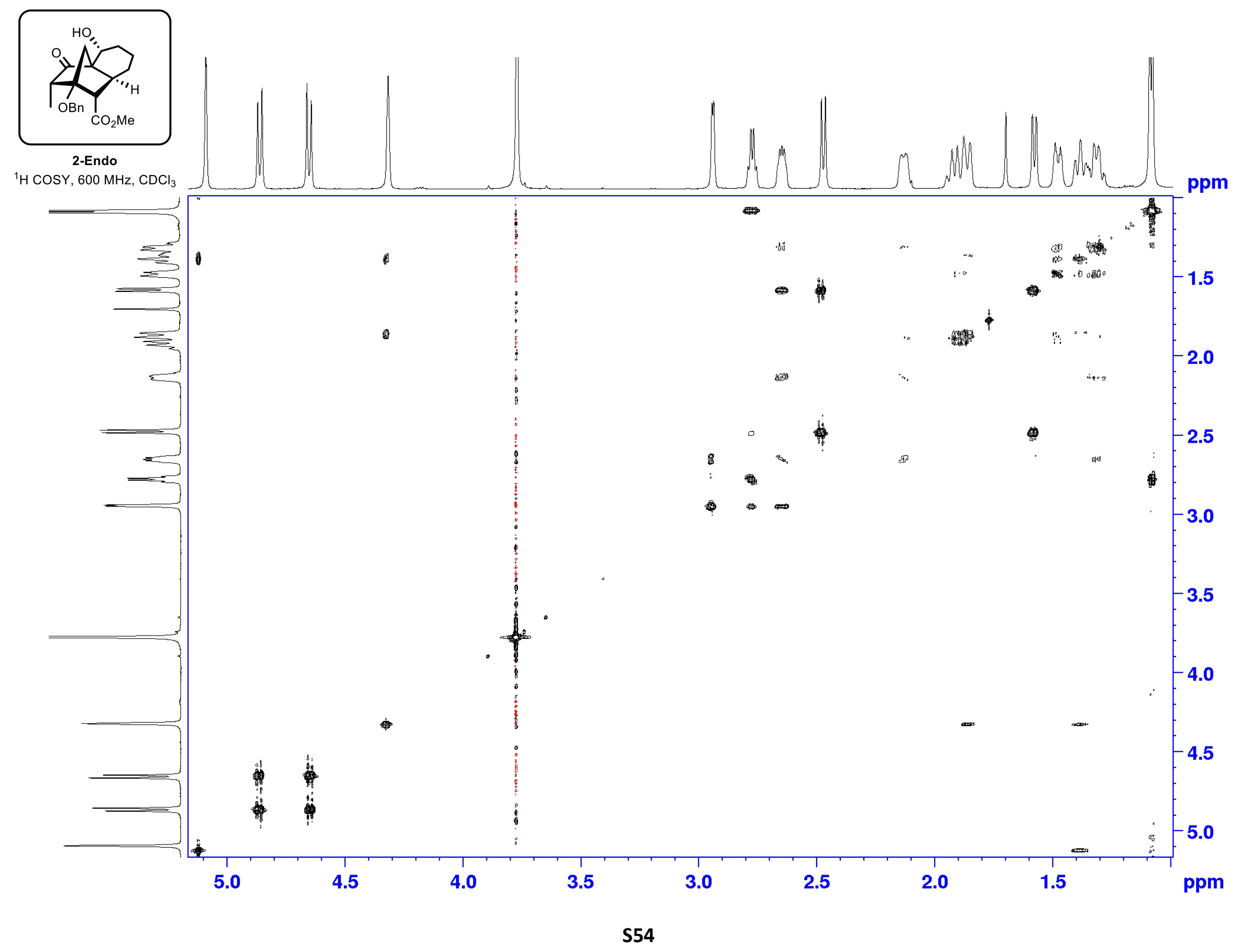




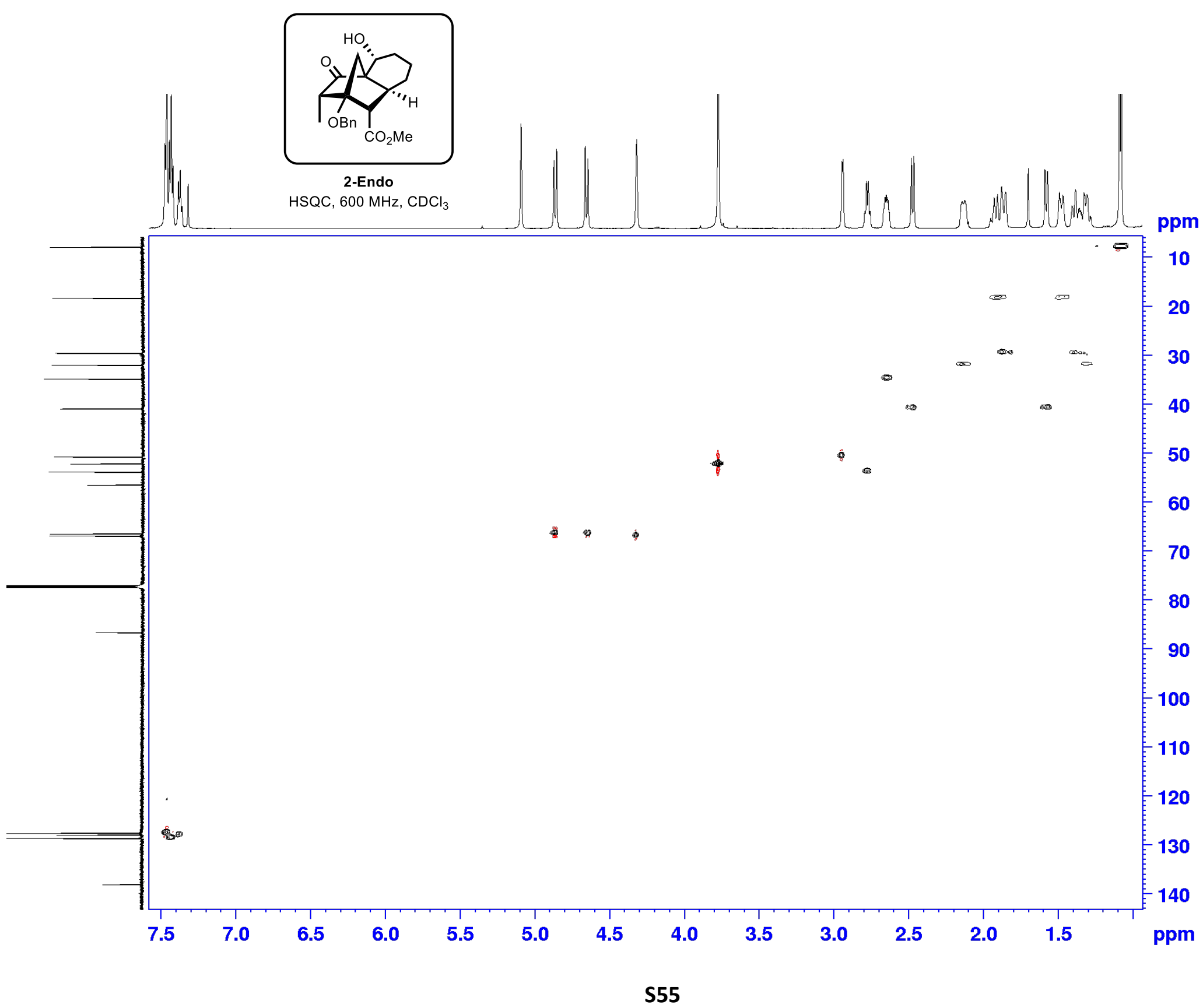




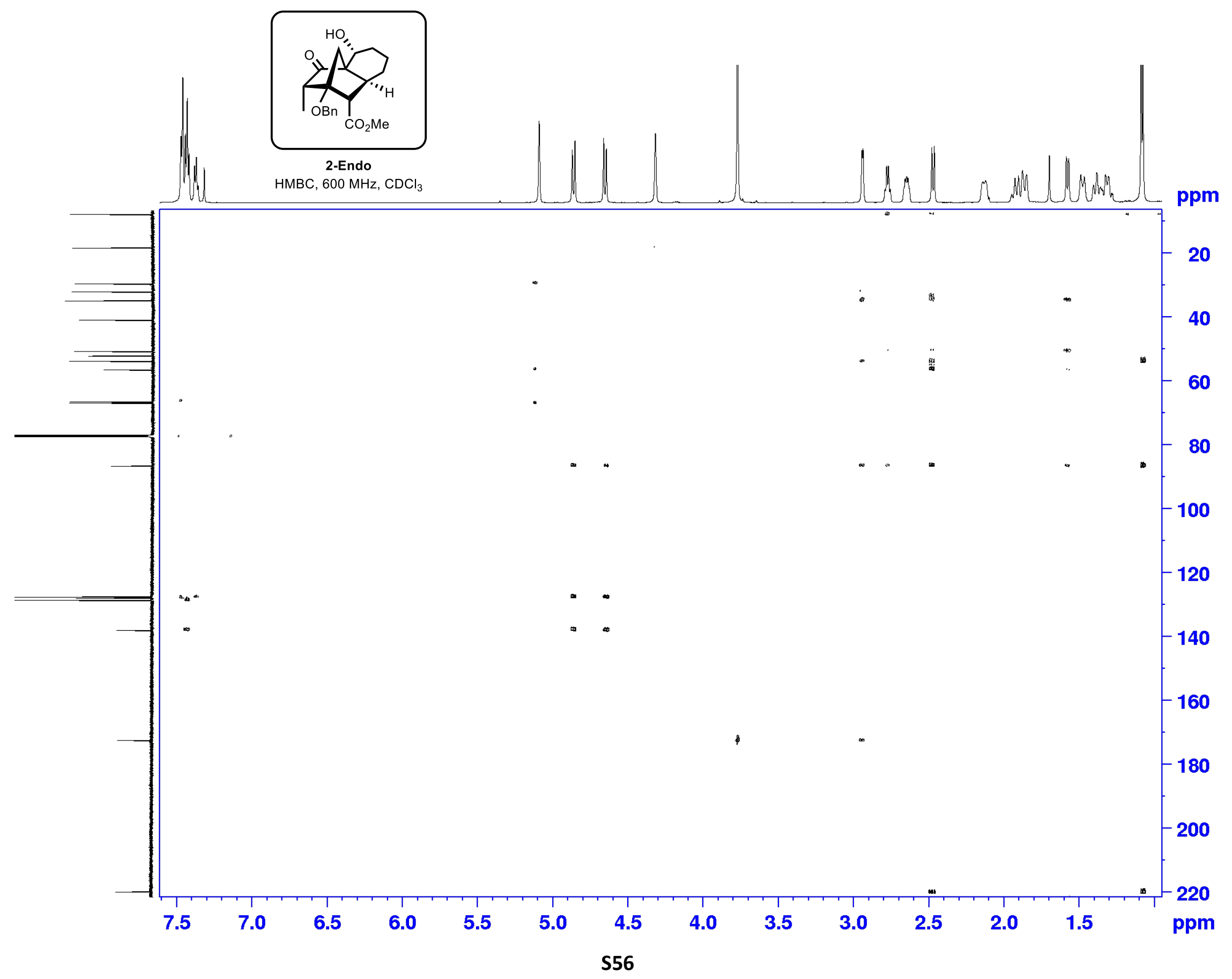




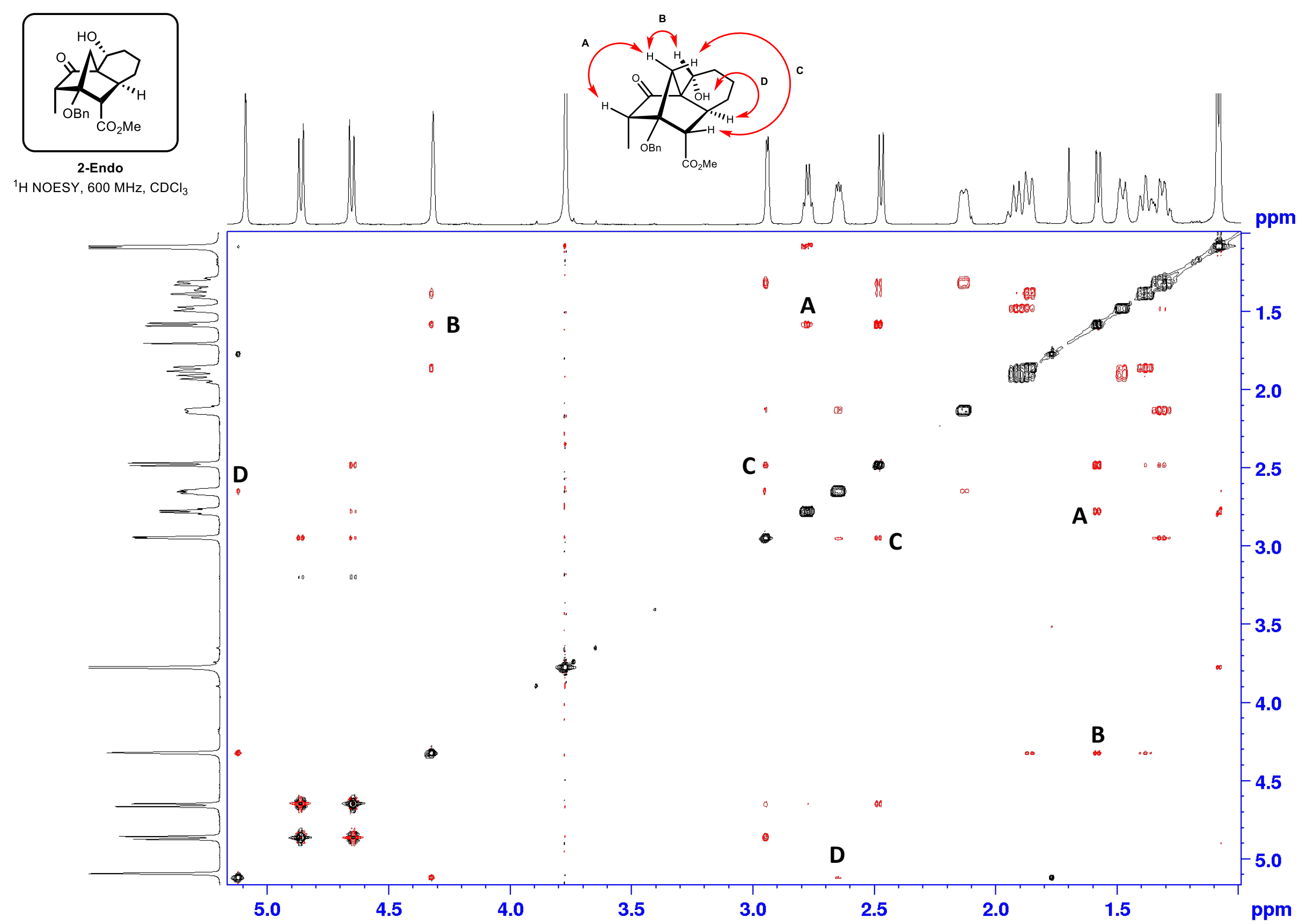




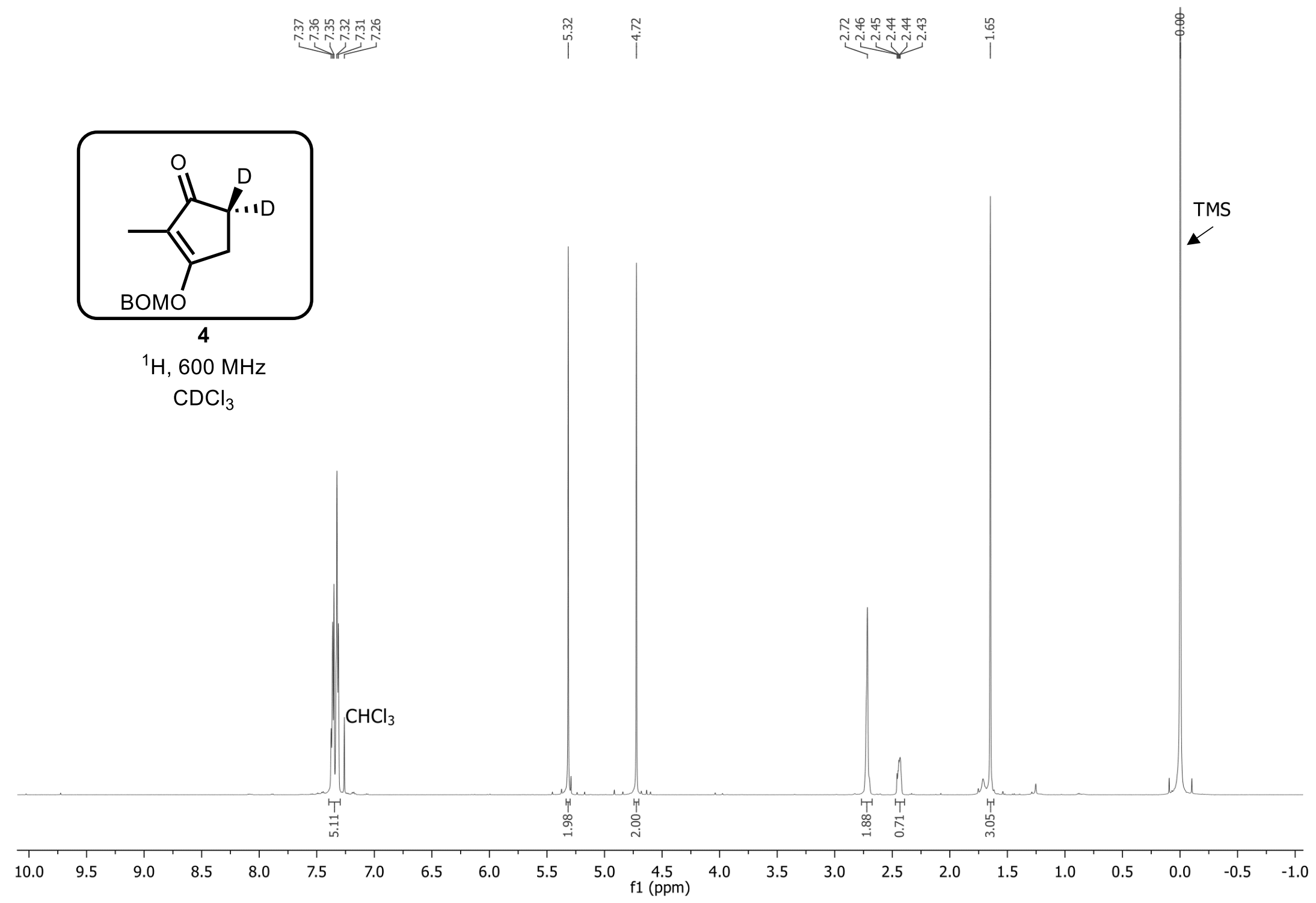




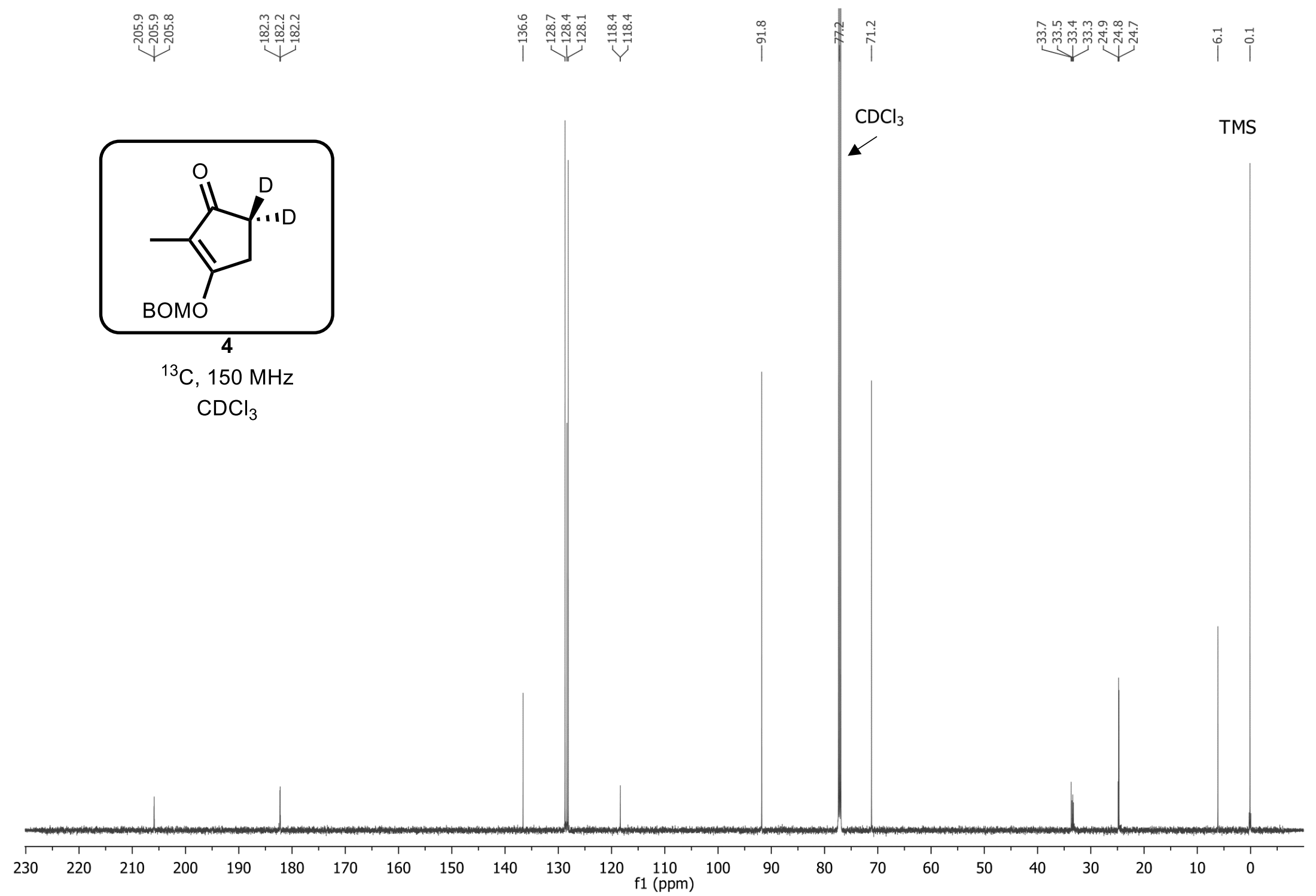




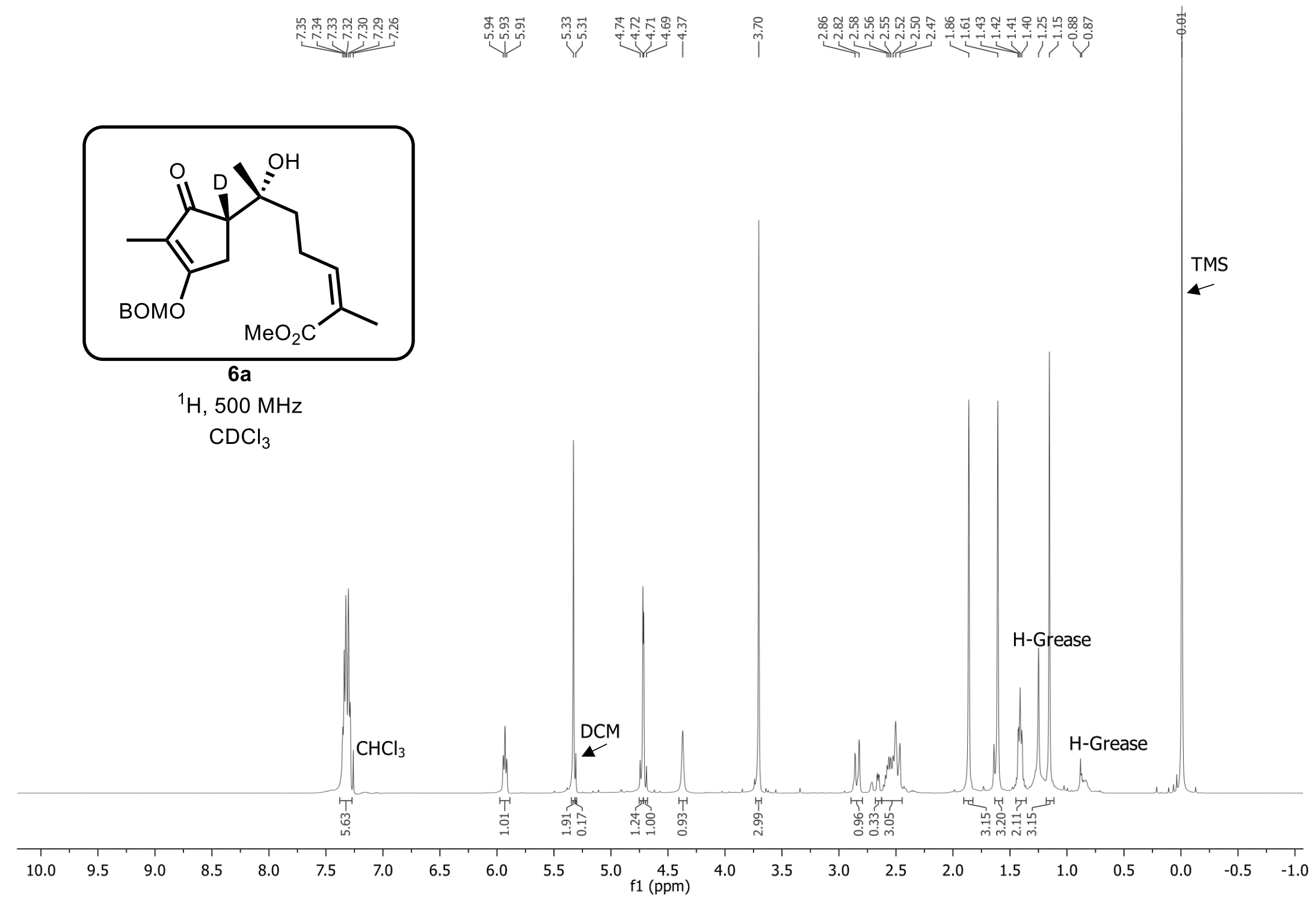




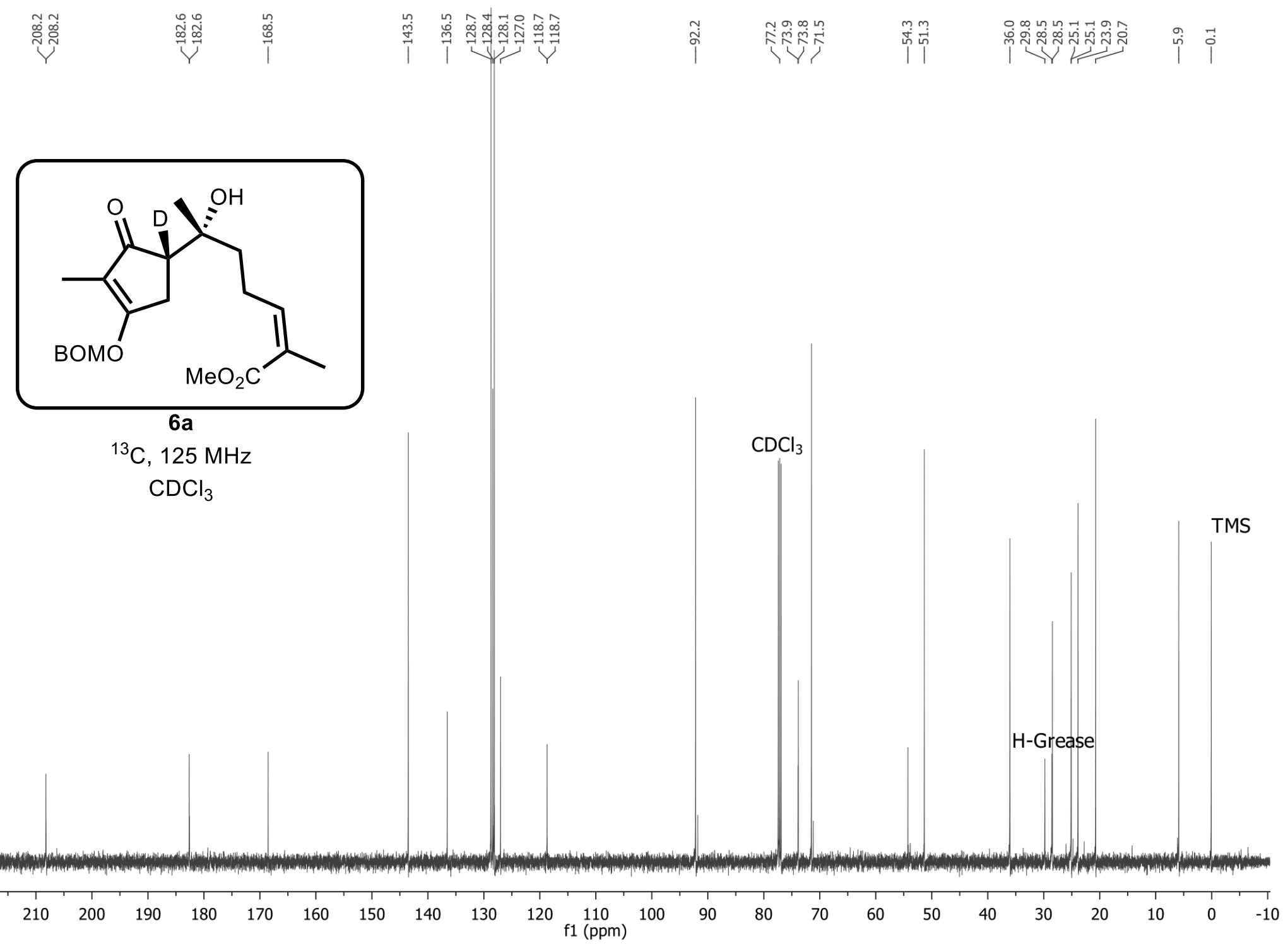




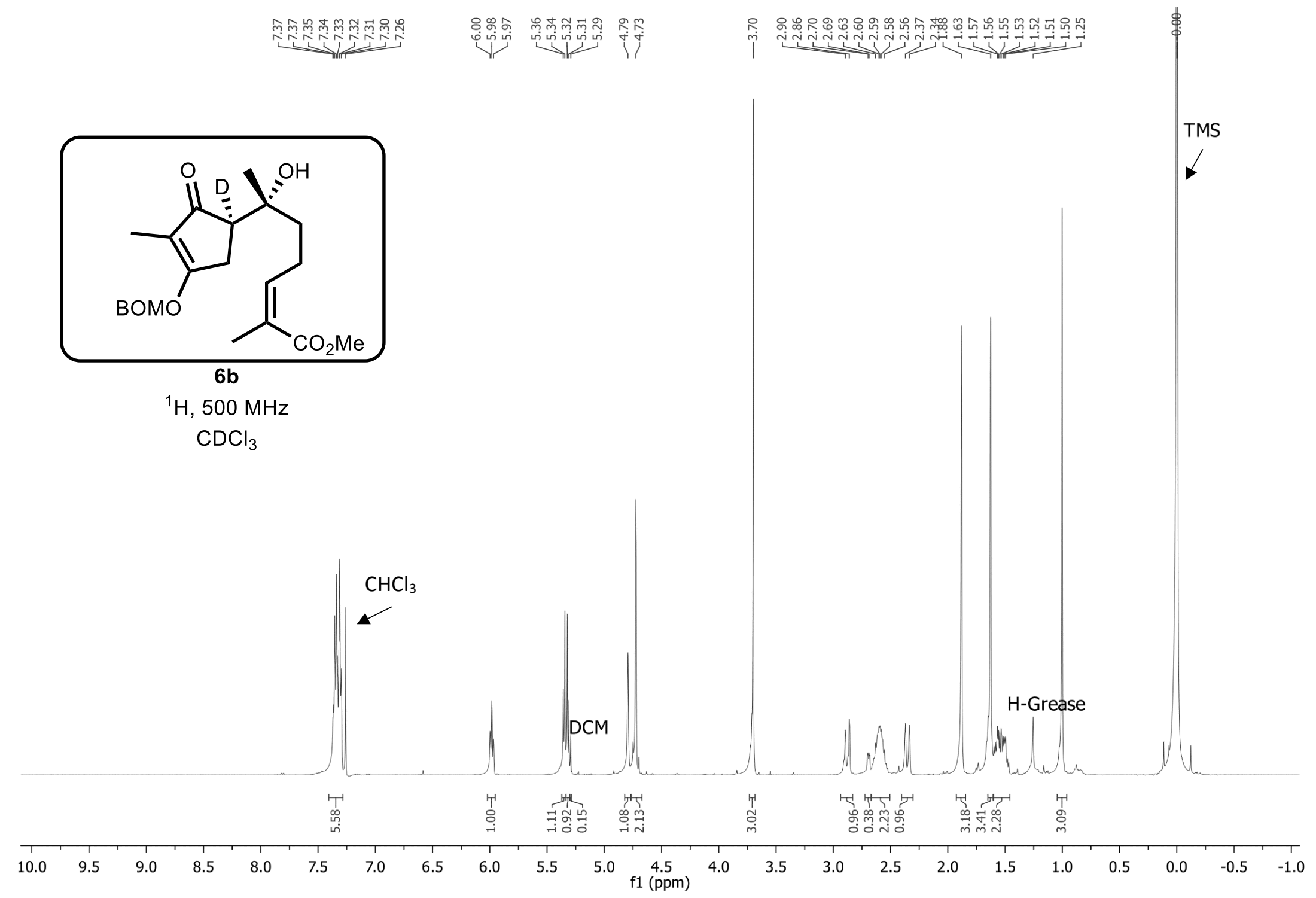




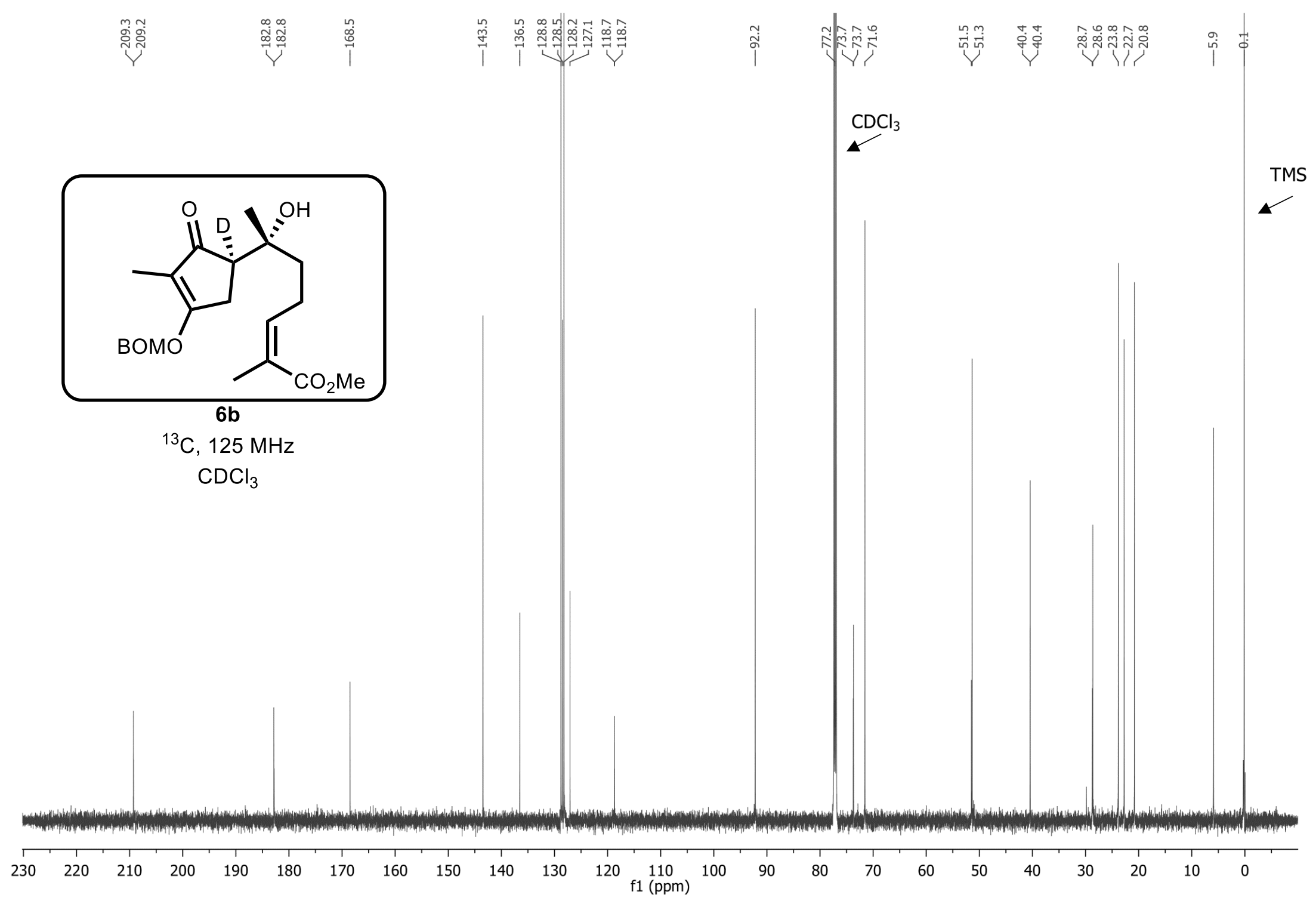




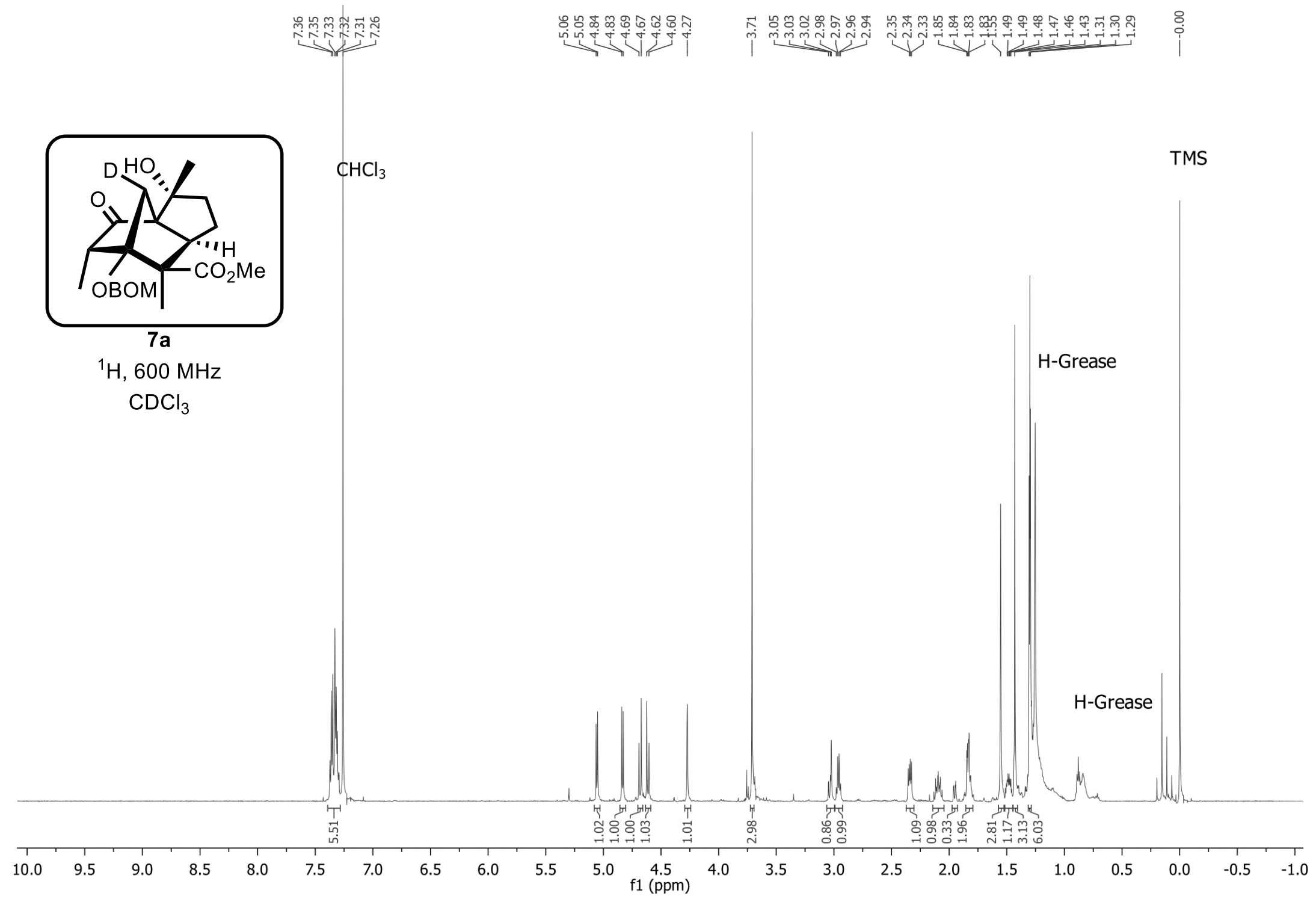




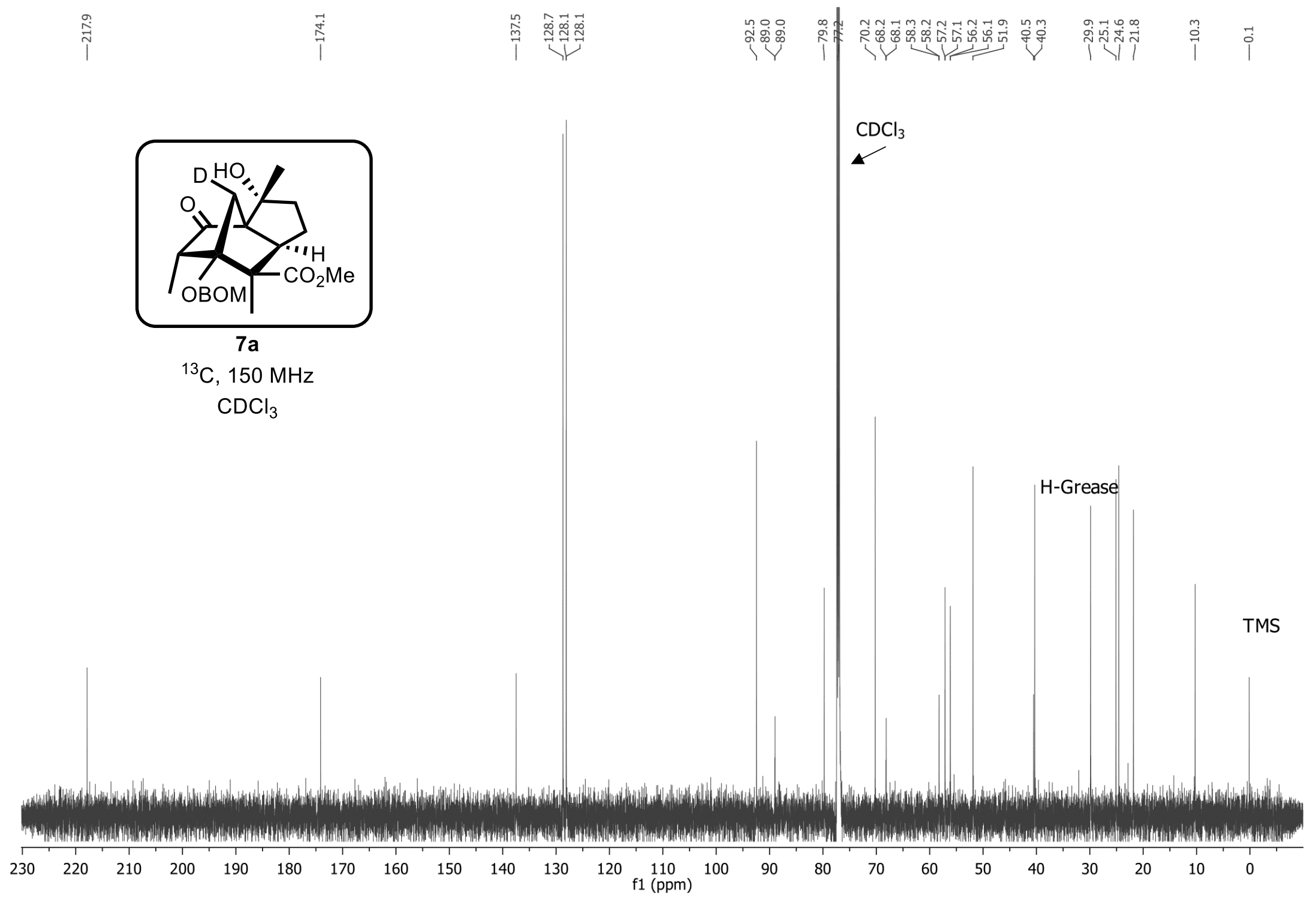




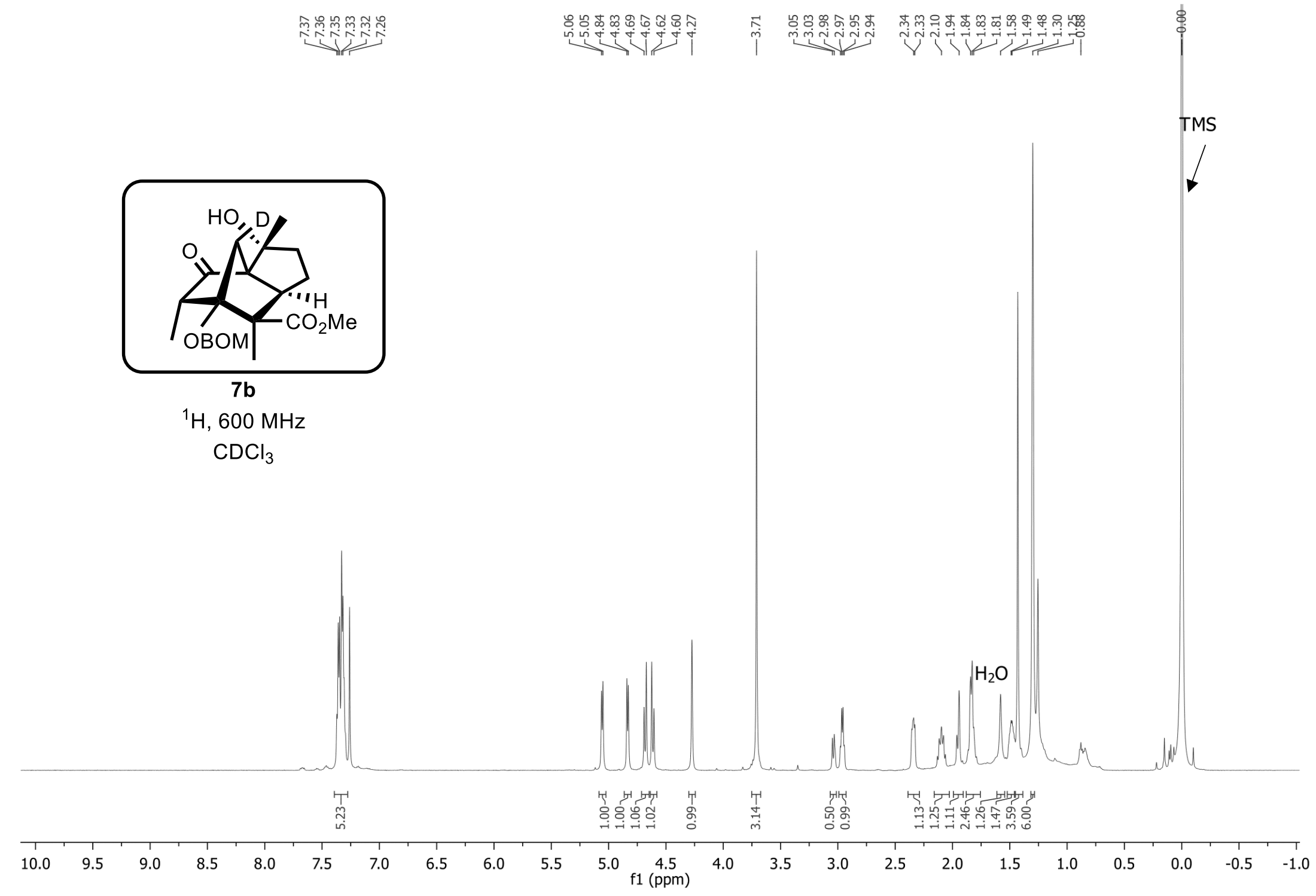




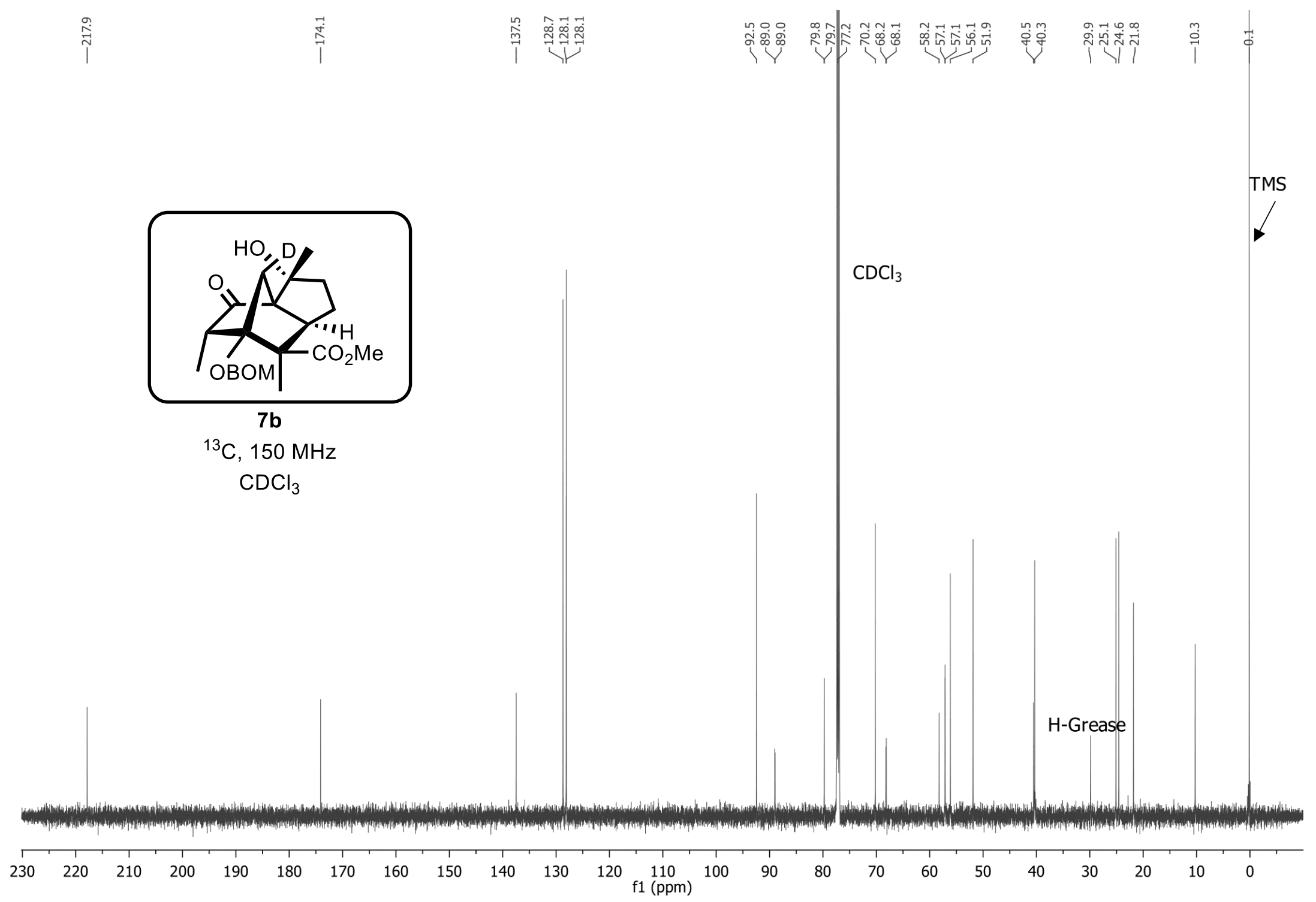




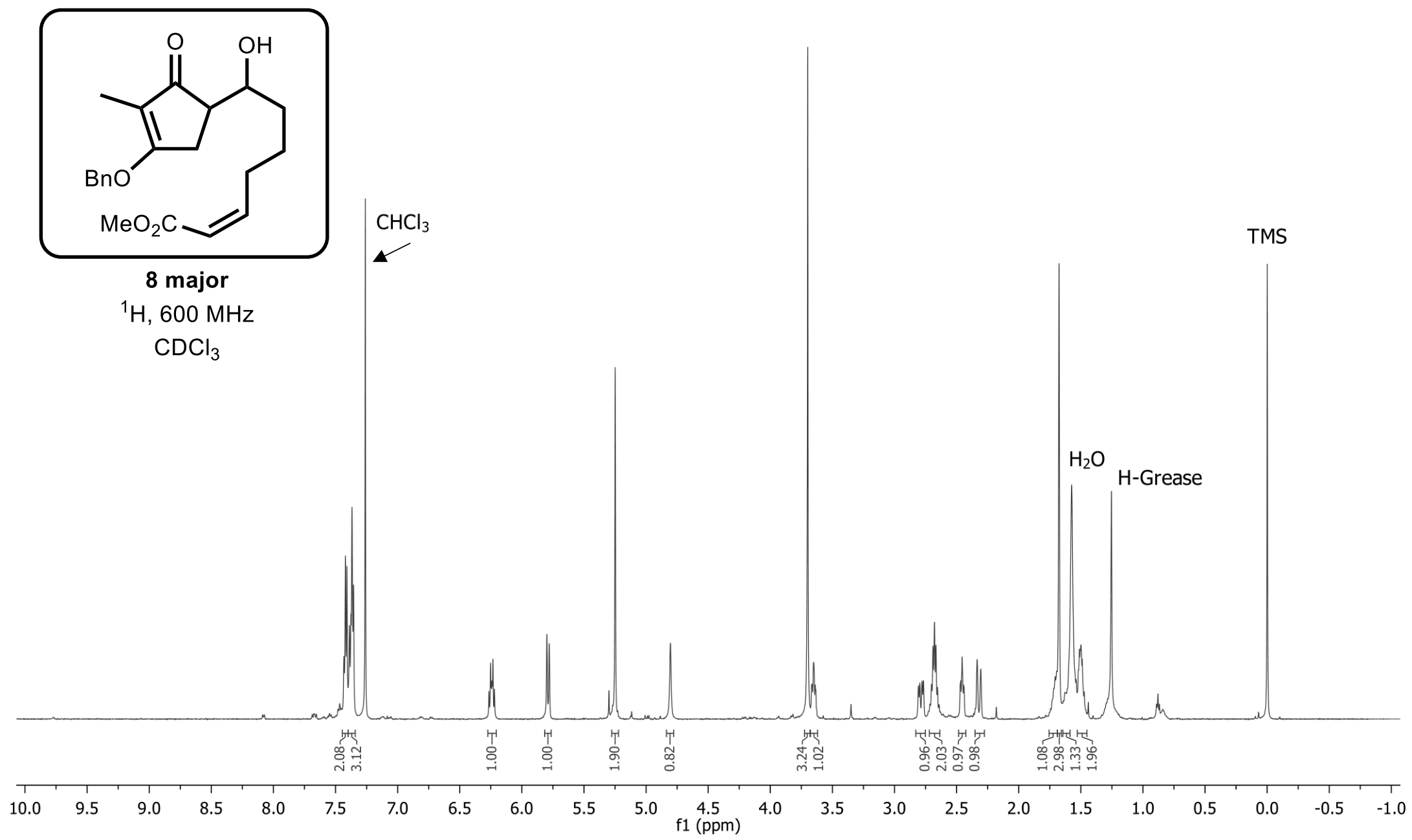




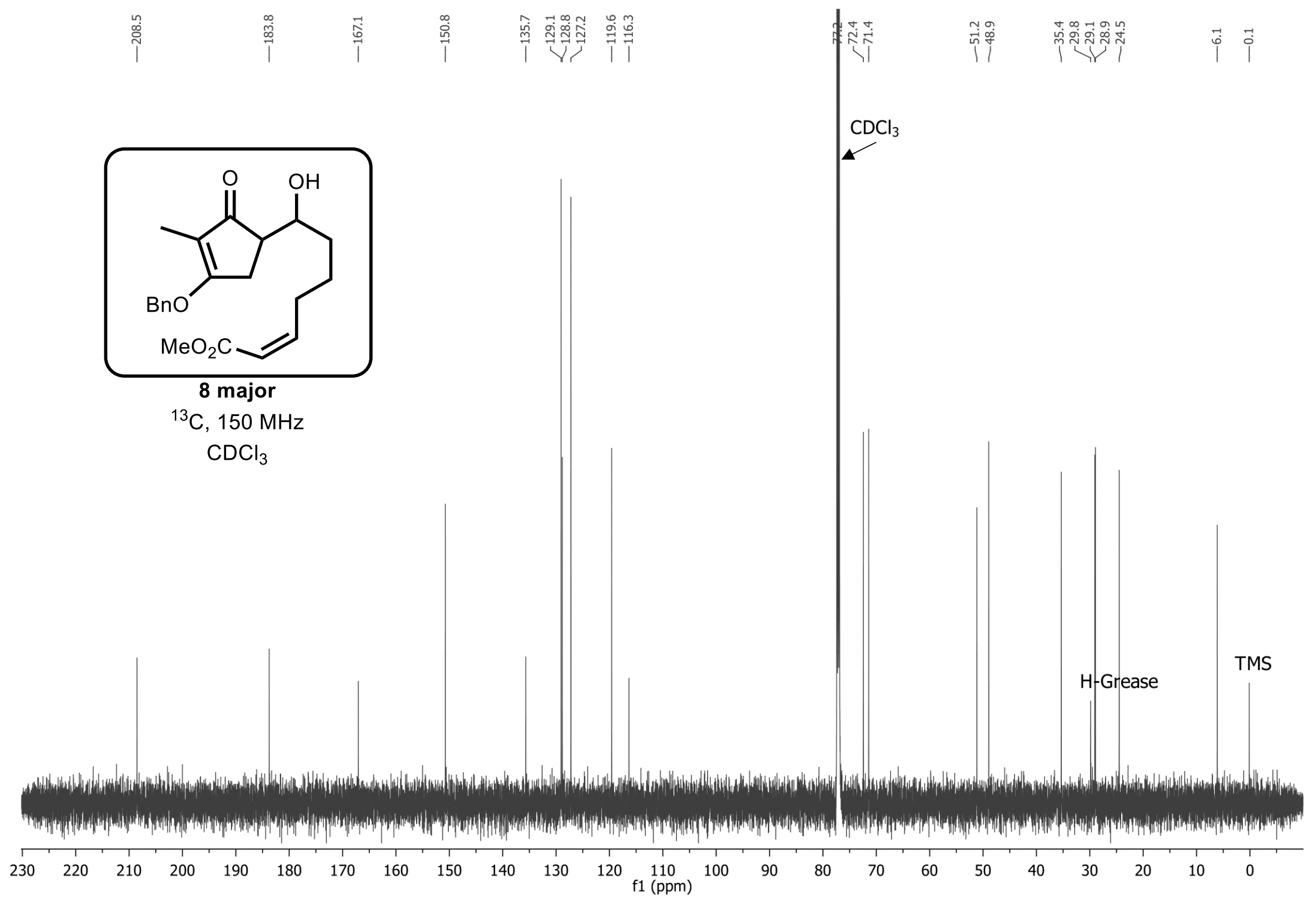




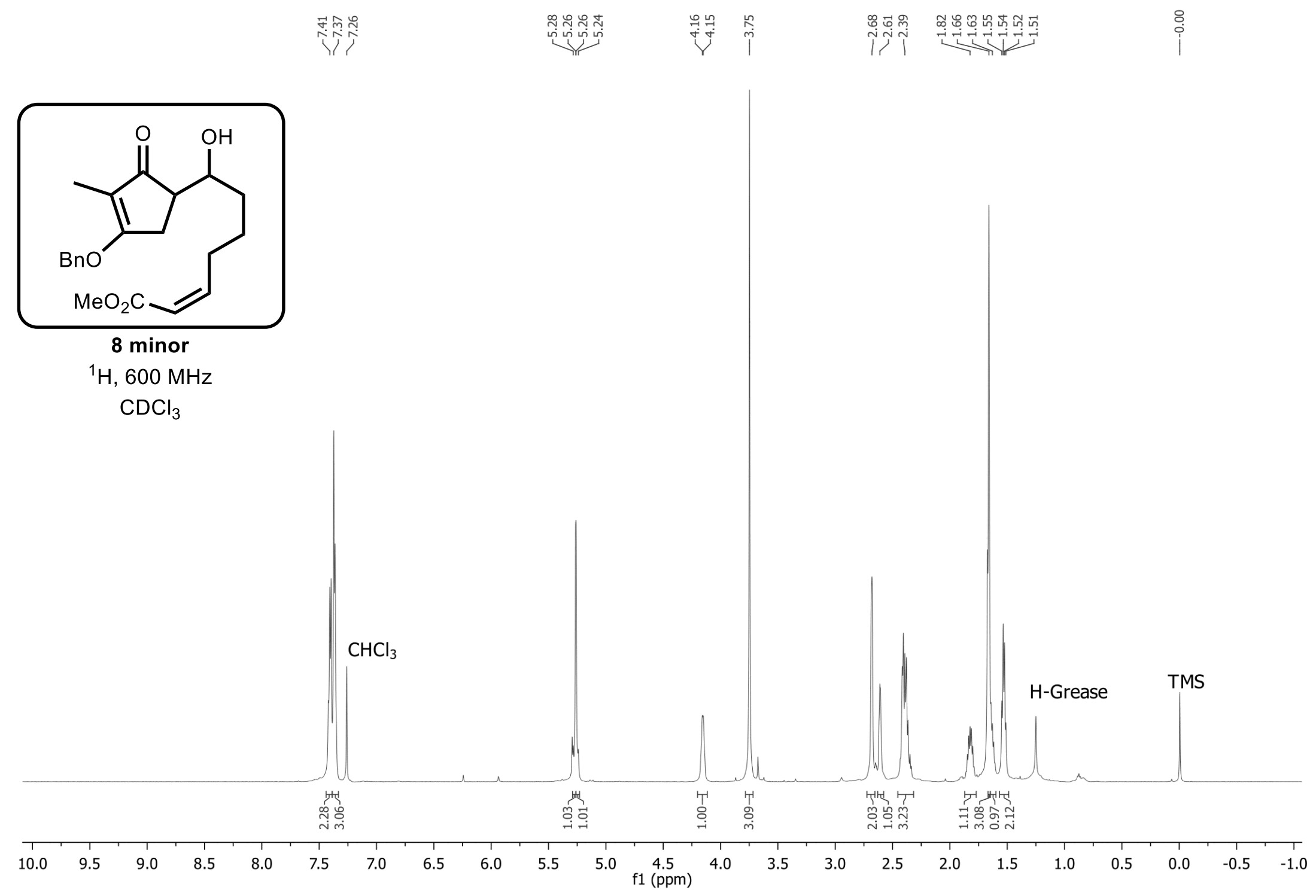




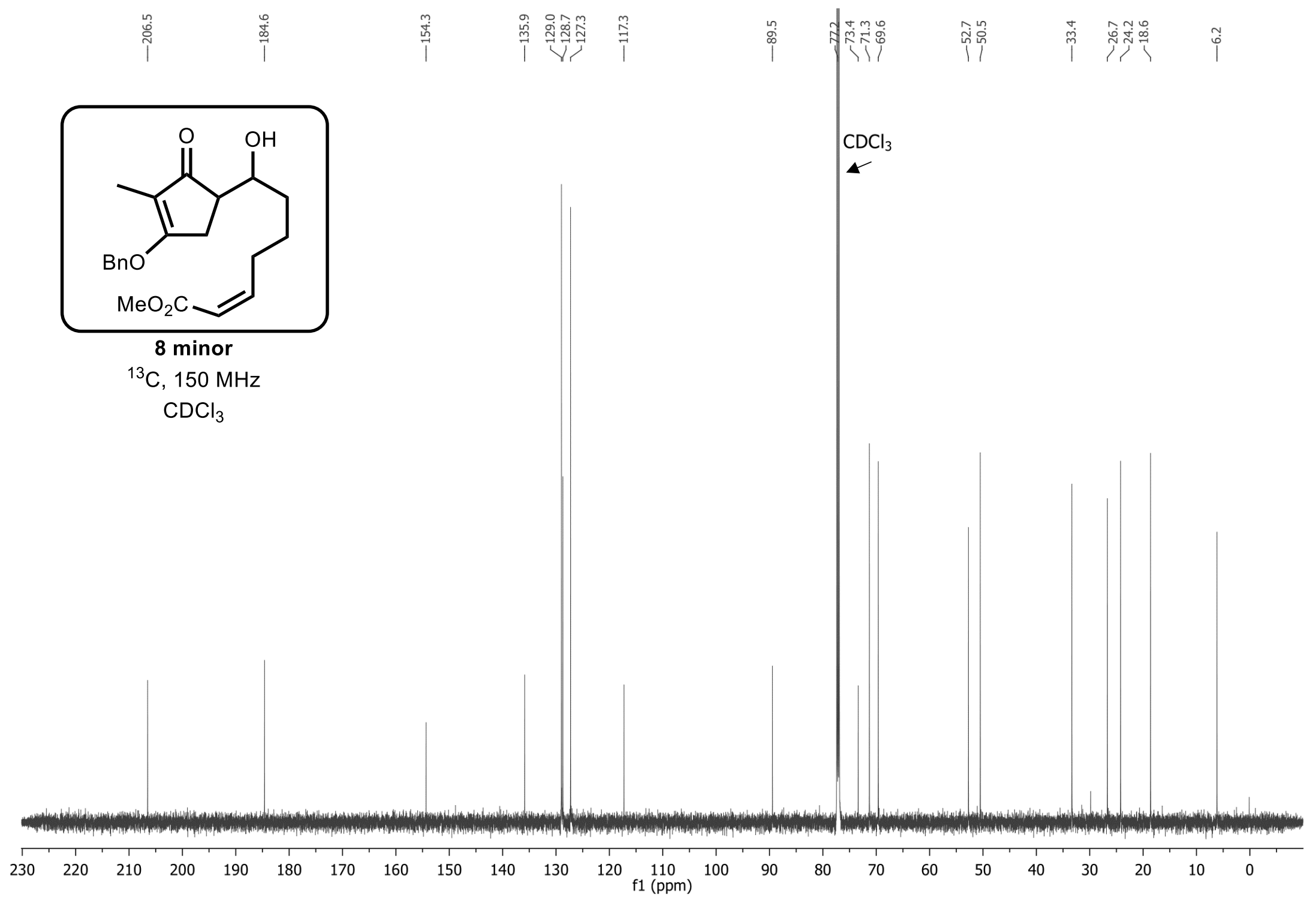




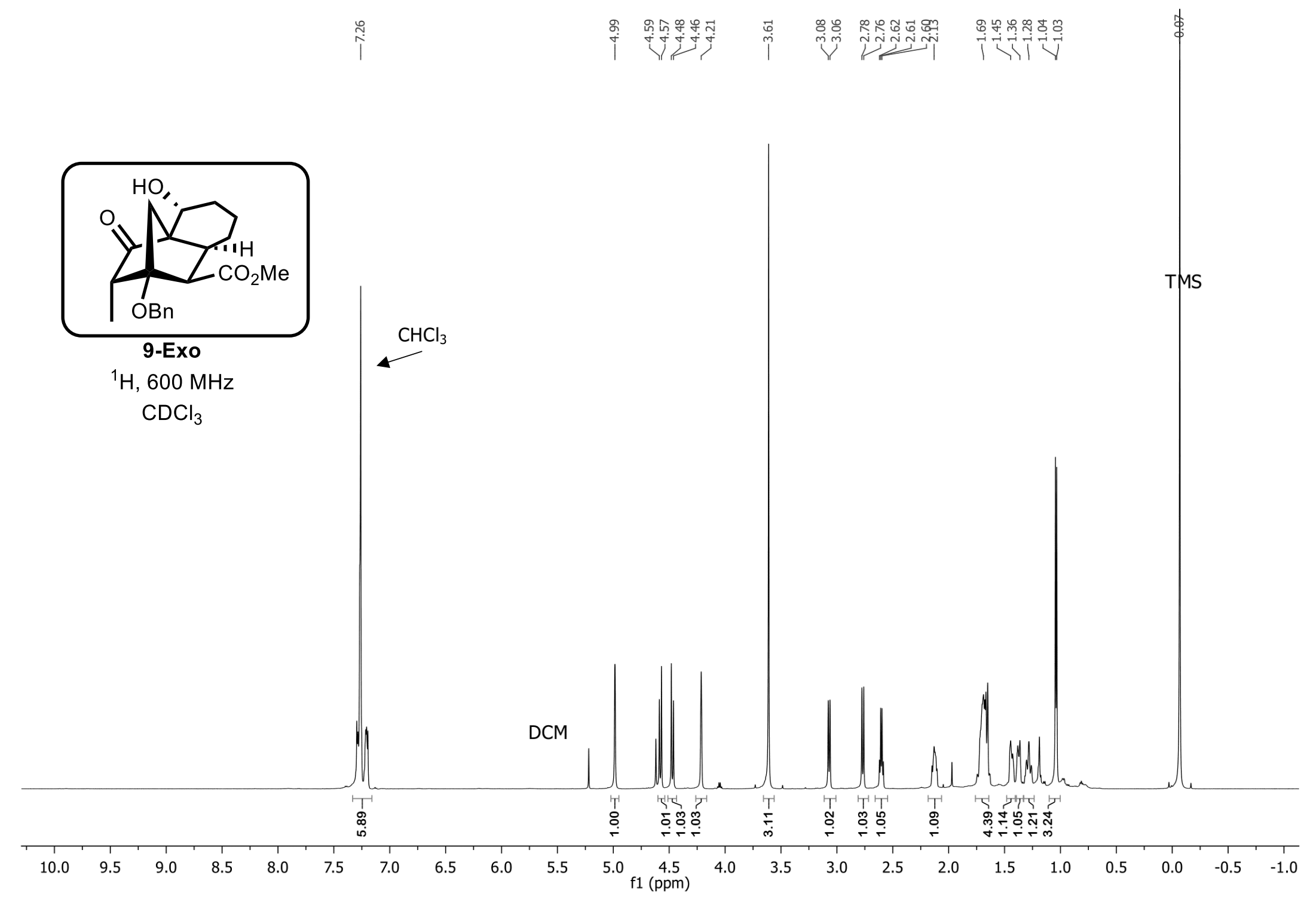




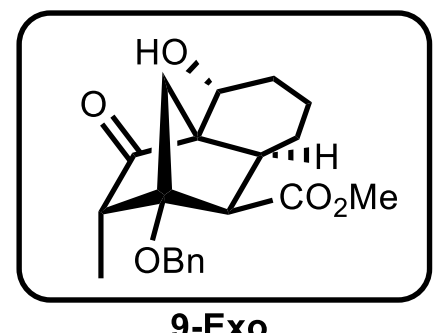

${ }^{3} \mathrm{C}, 150 \mathrm{MHz}$ $\mathrm{CDCl}_{3}$

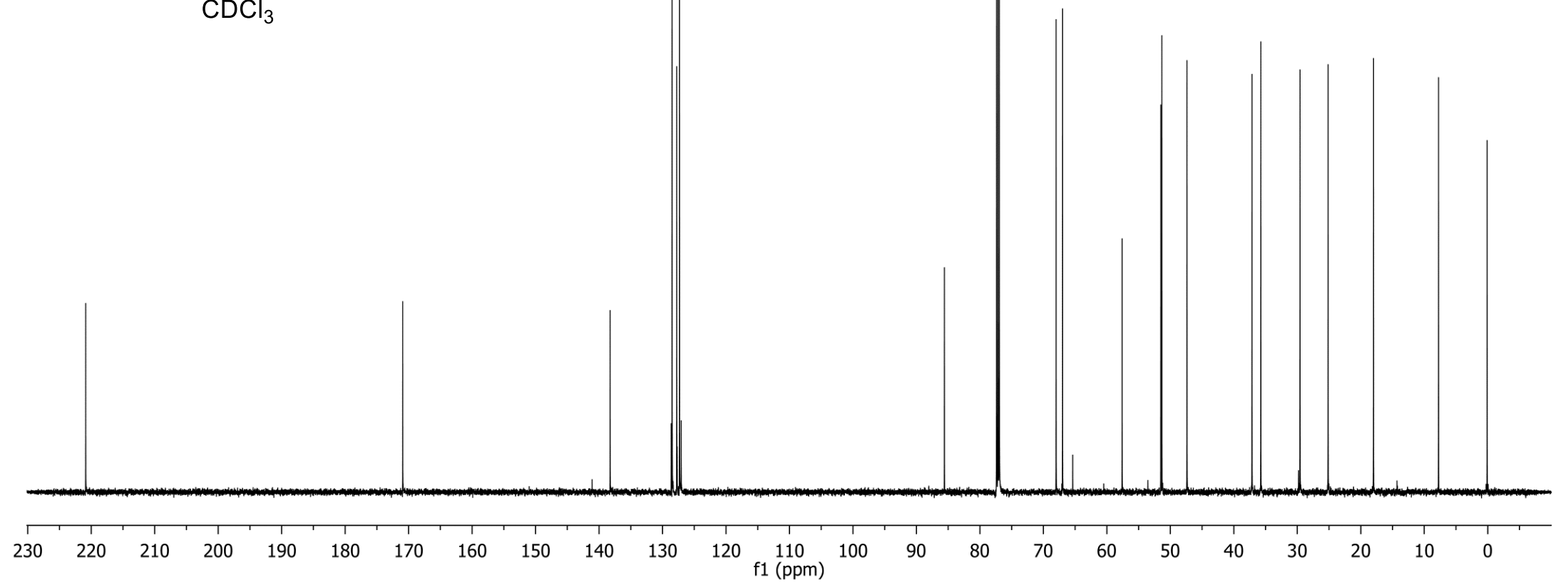




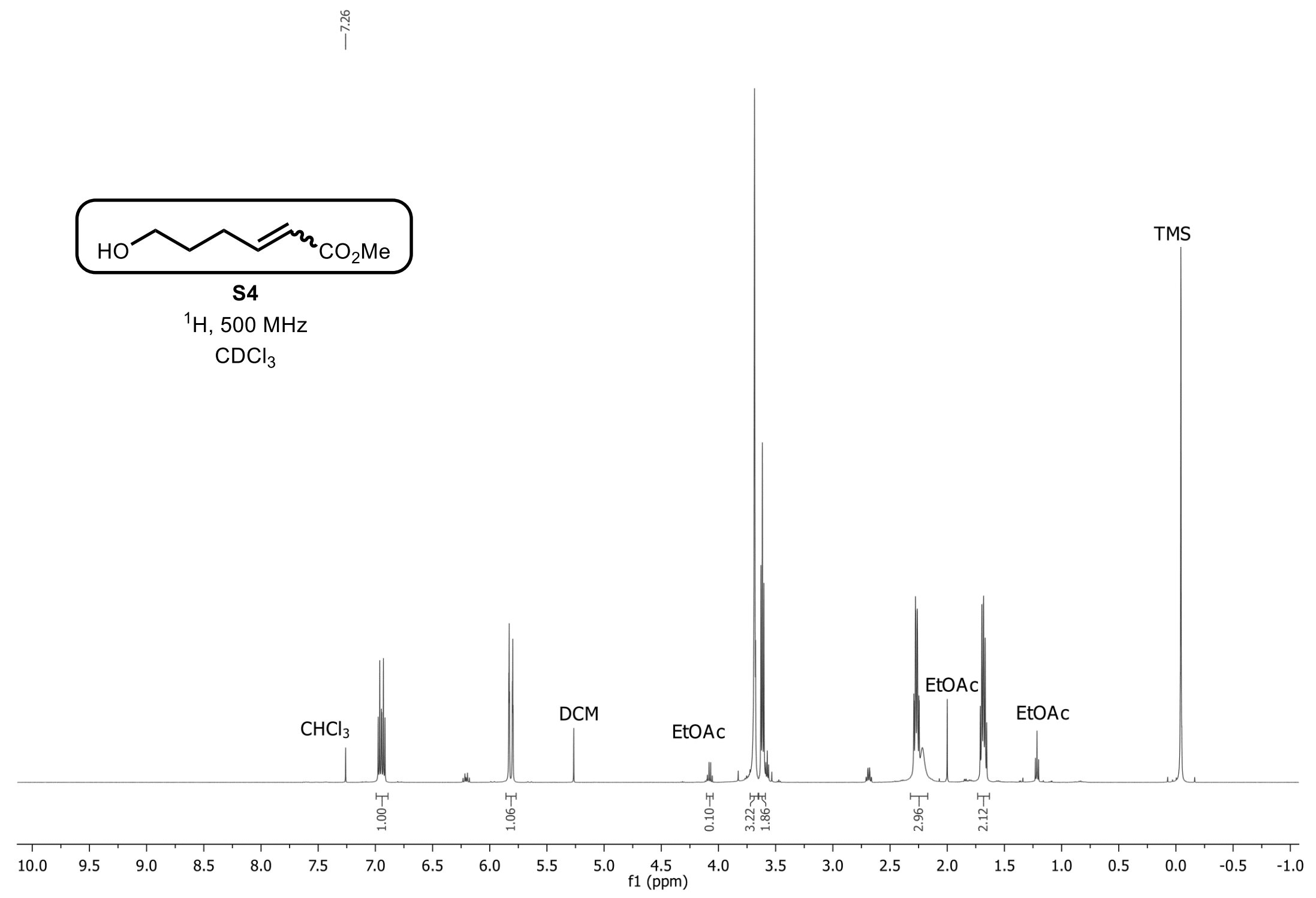




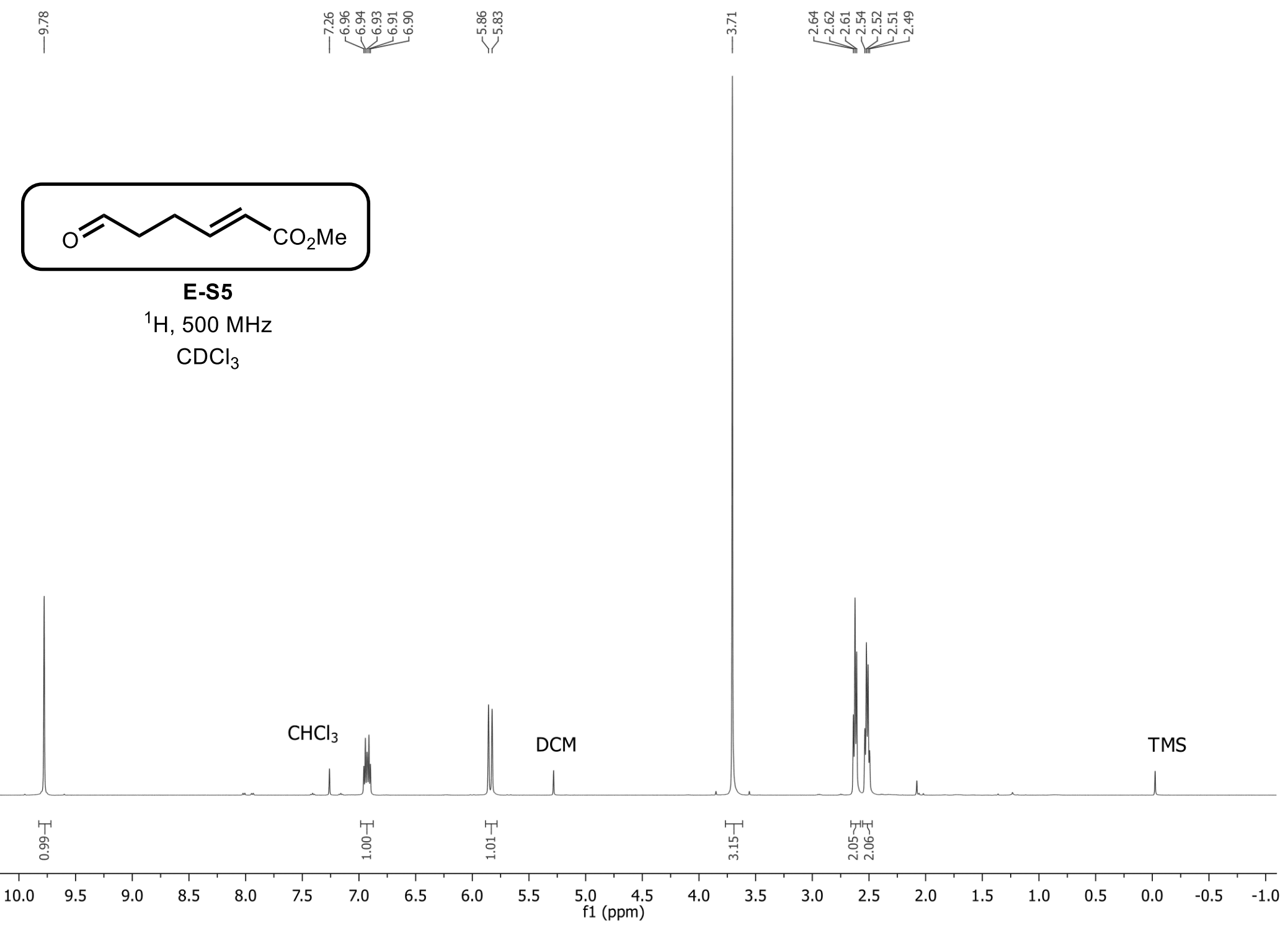




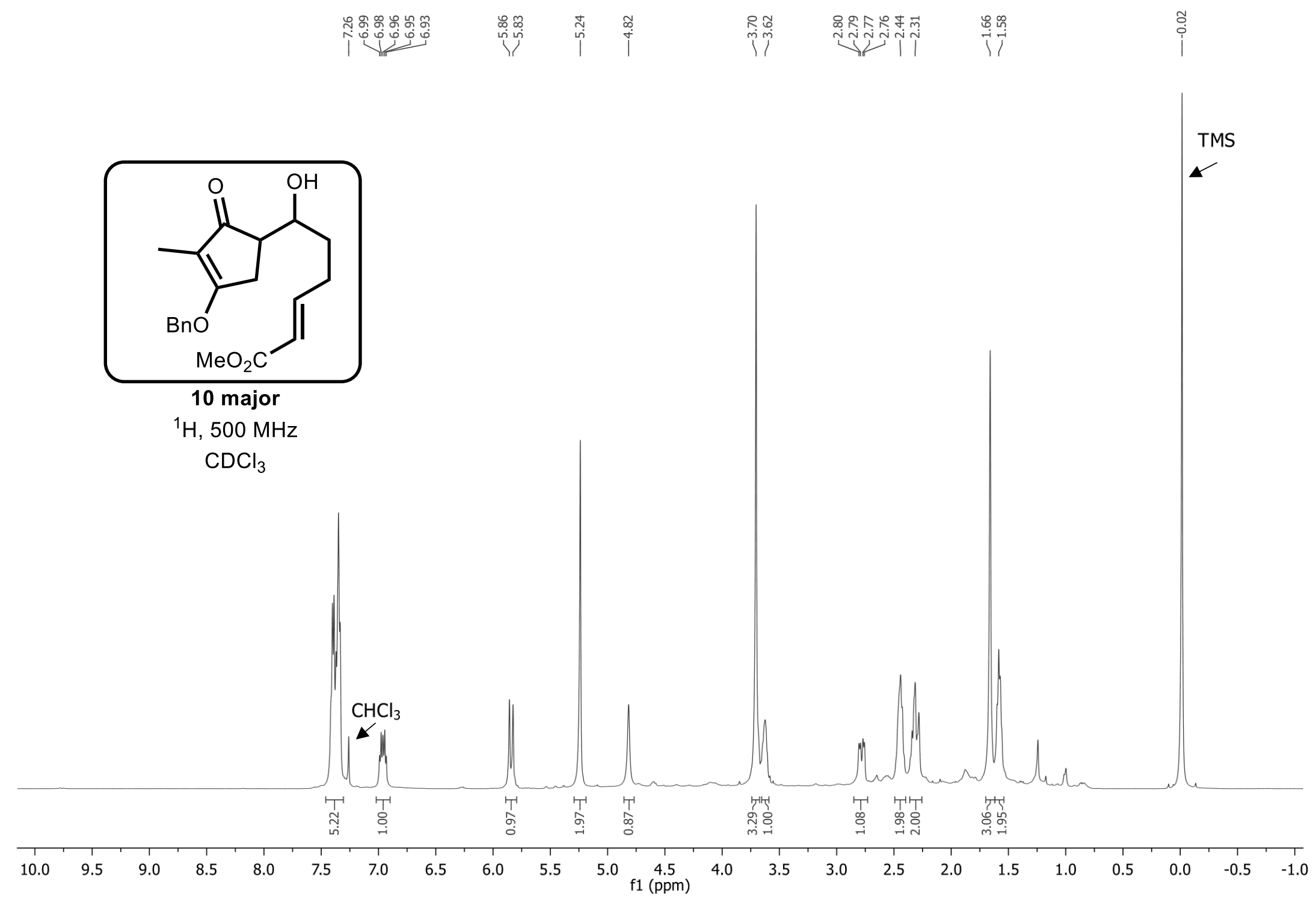




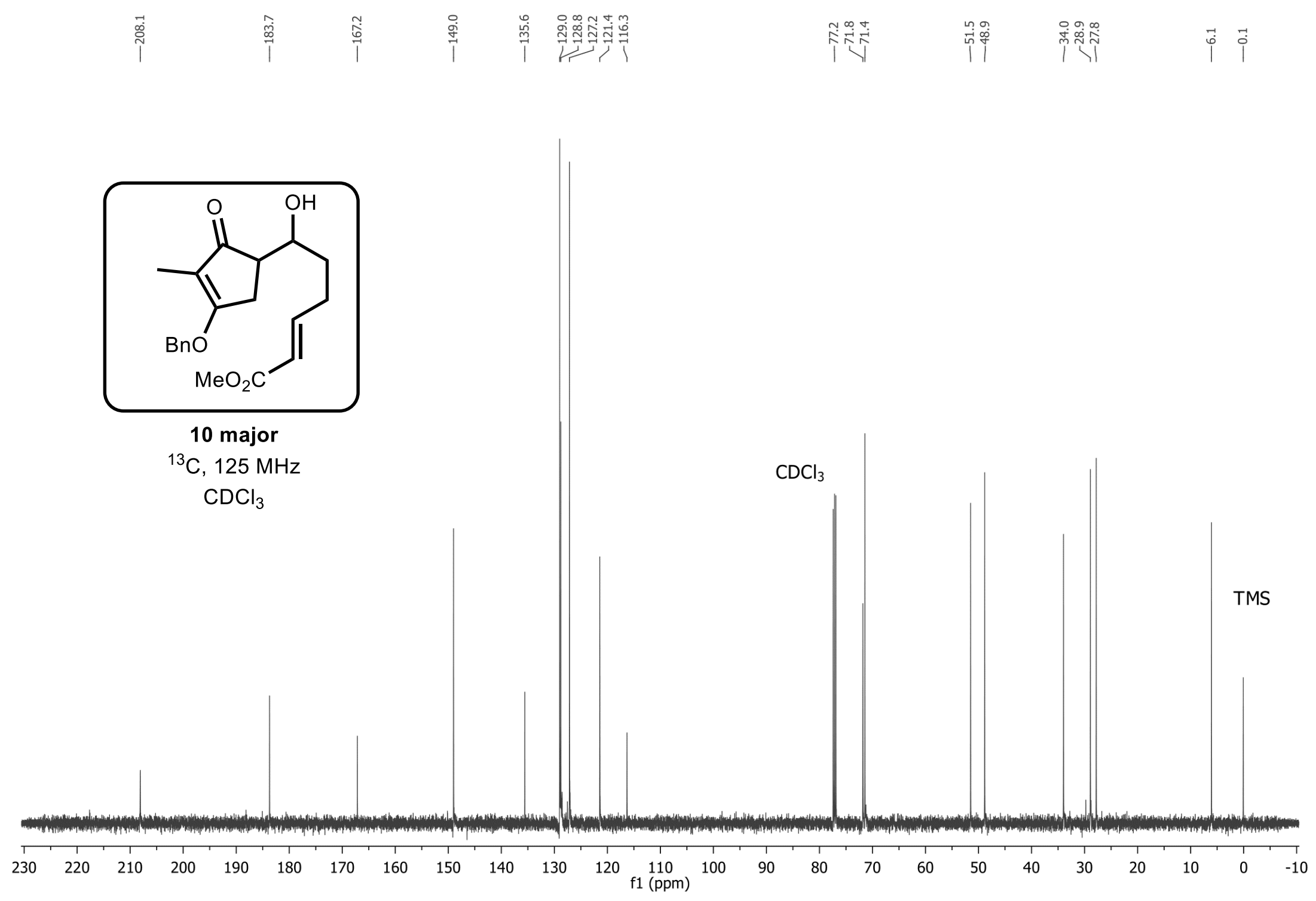




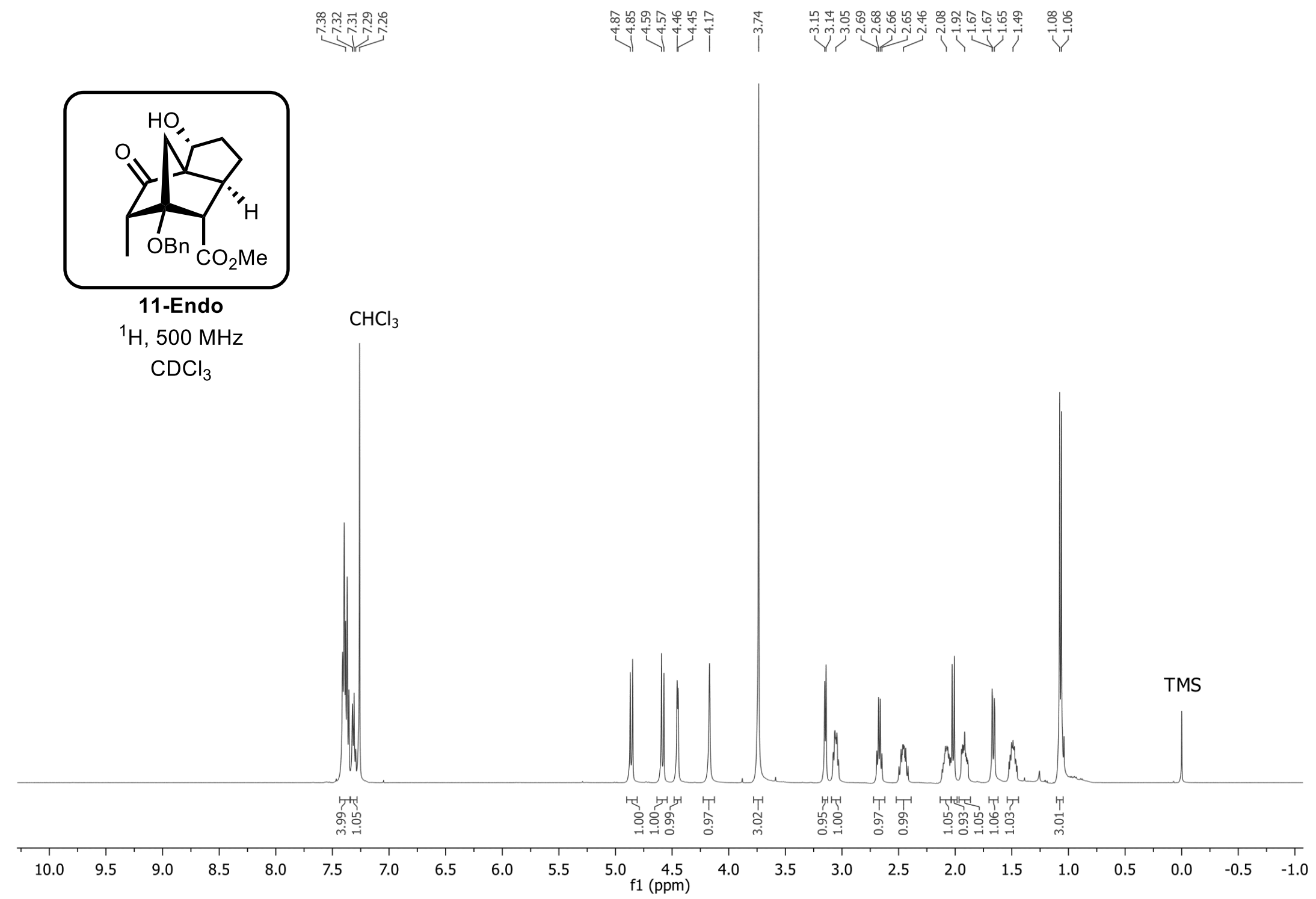



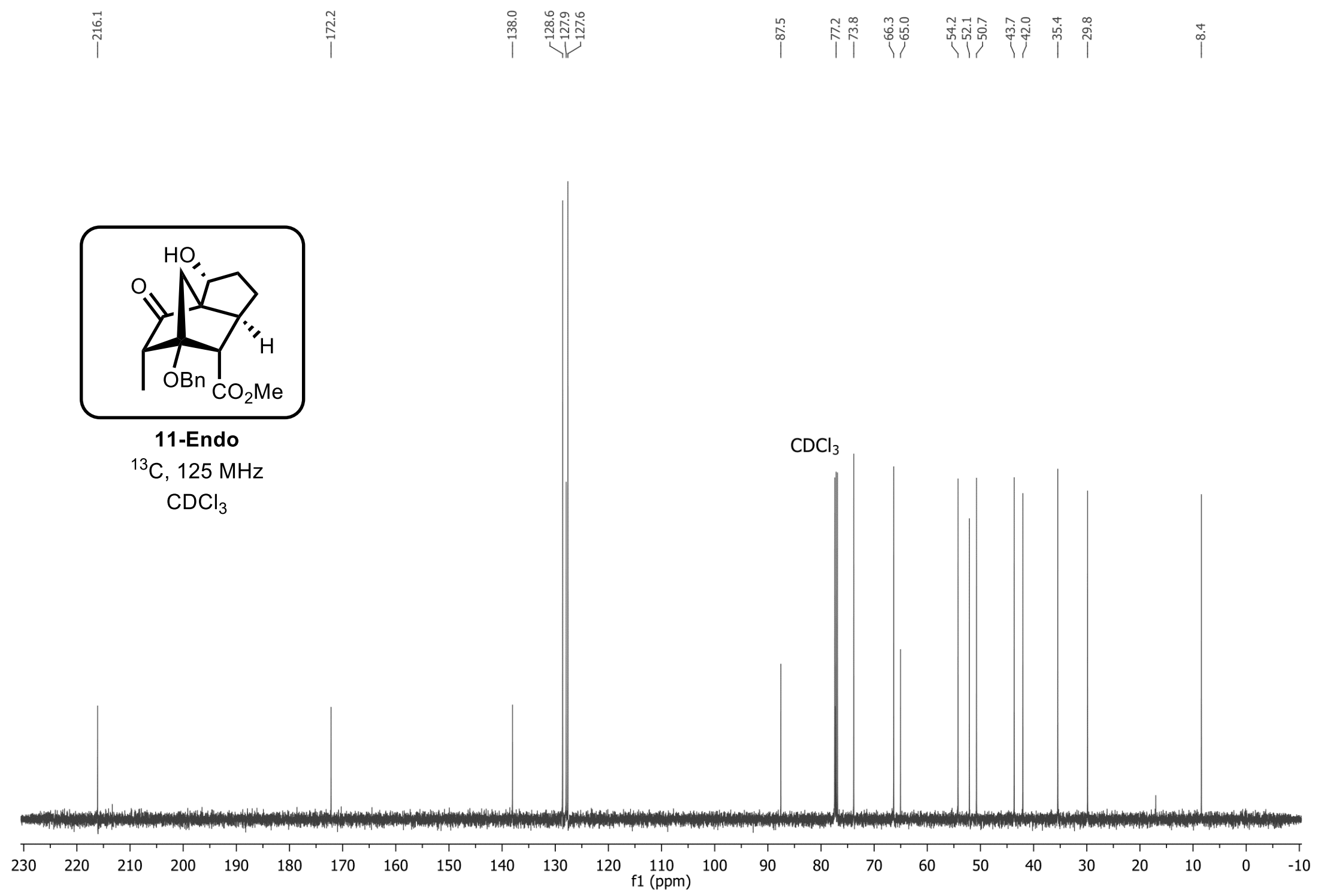


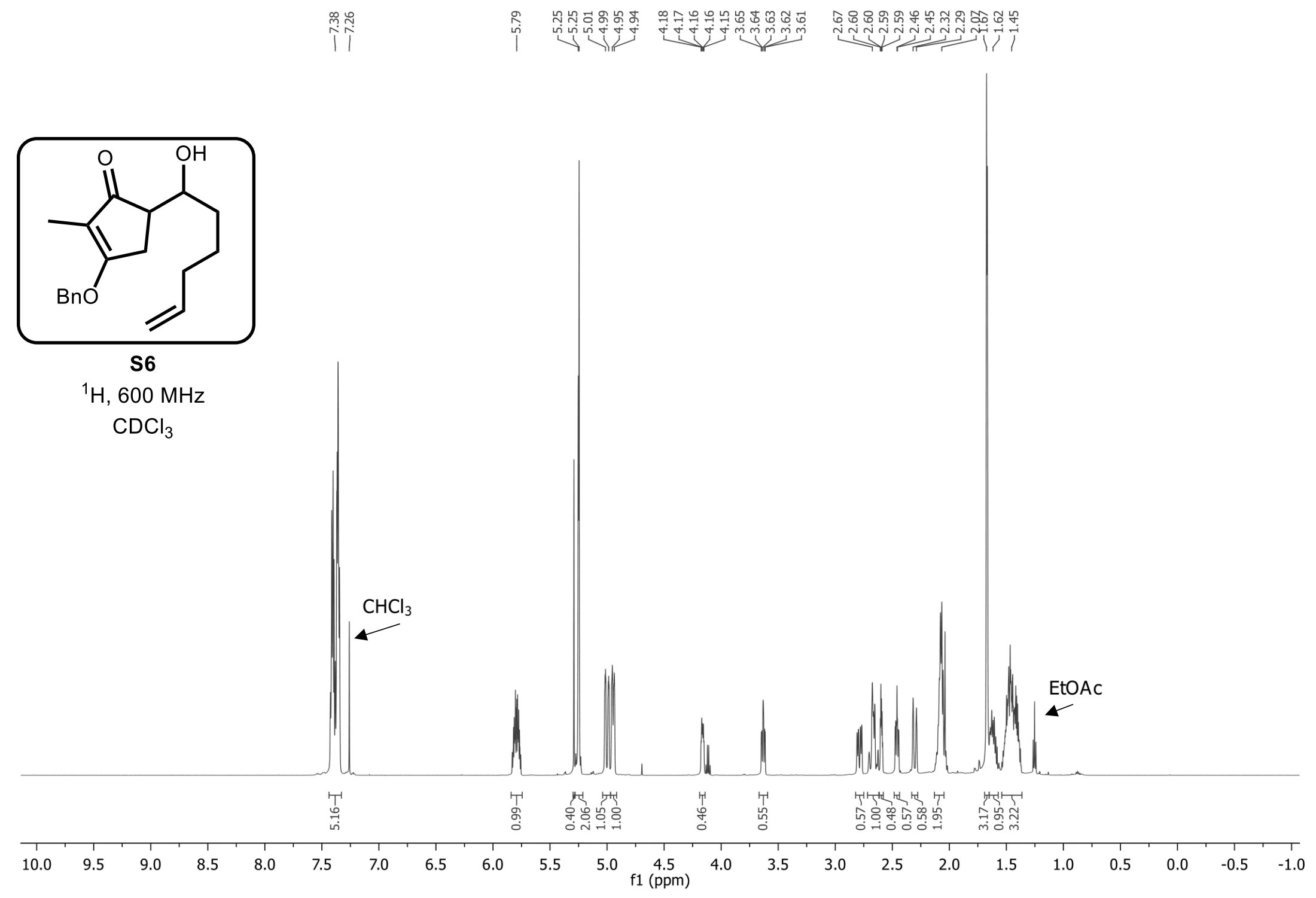




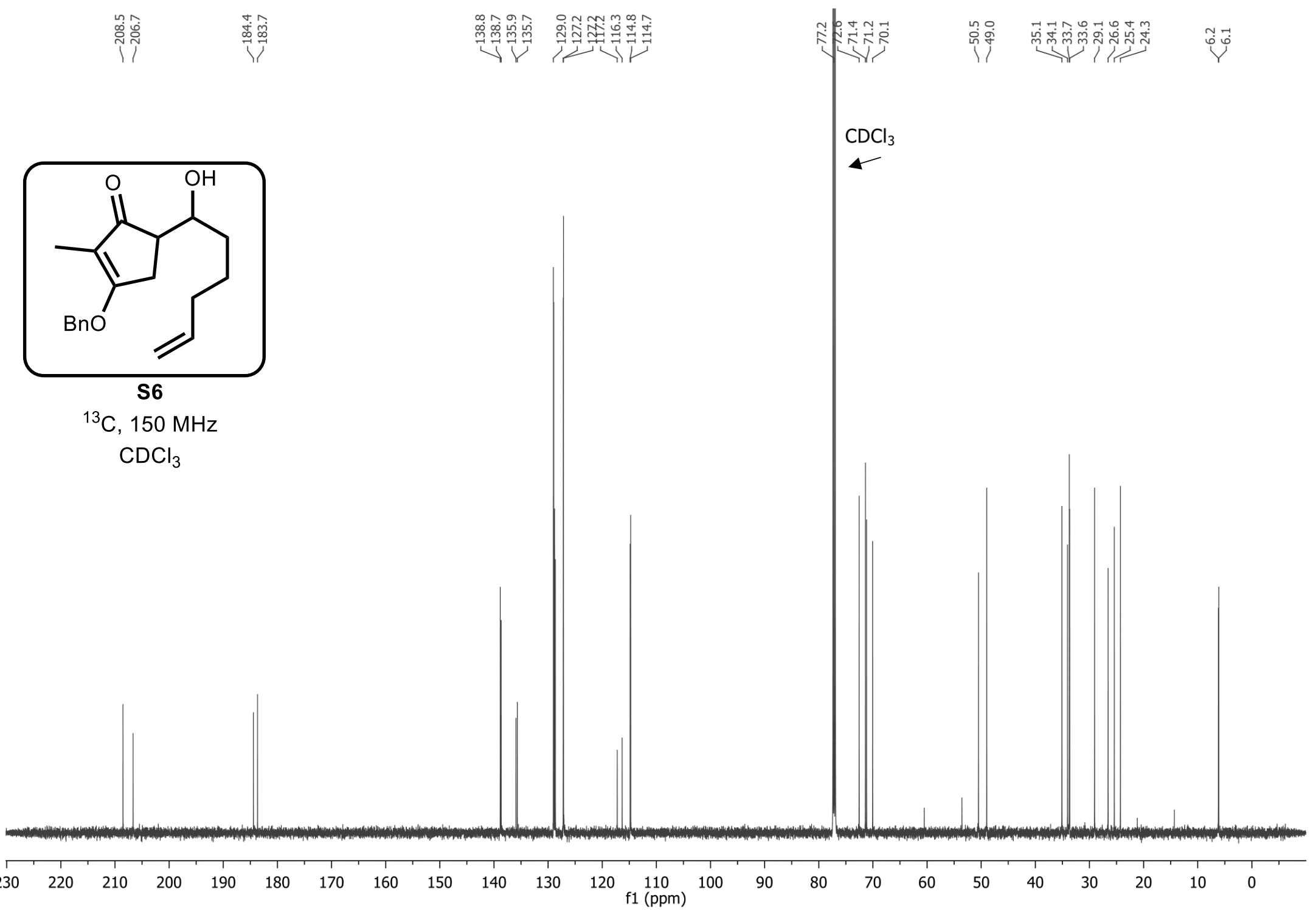



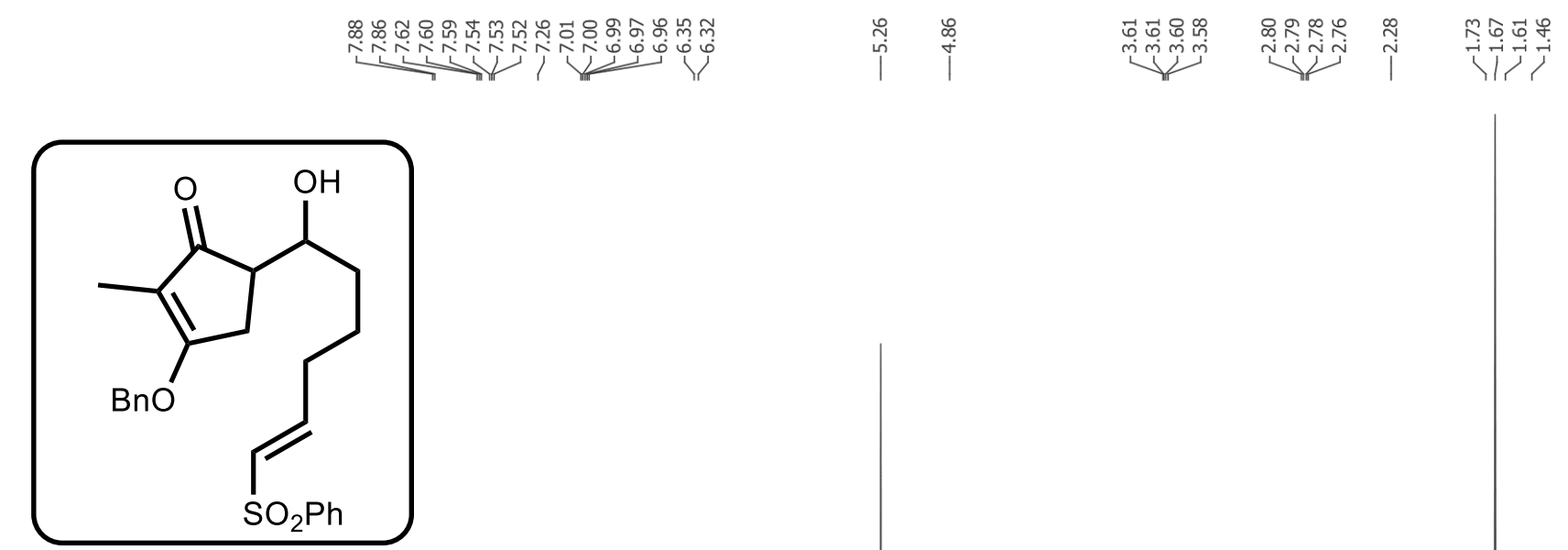

12 major

${ }^{1} \mathrm{H}, 600 \mathrm{MHz}$ $\mathrm{CDCl}_{3}$

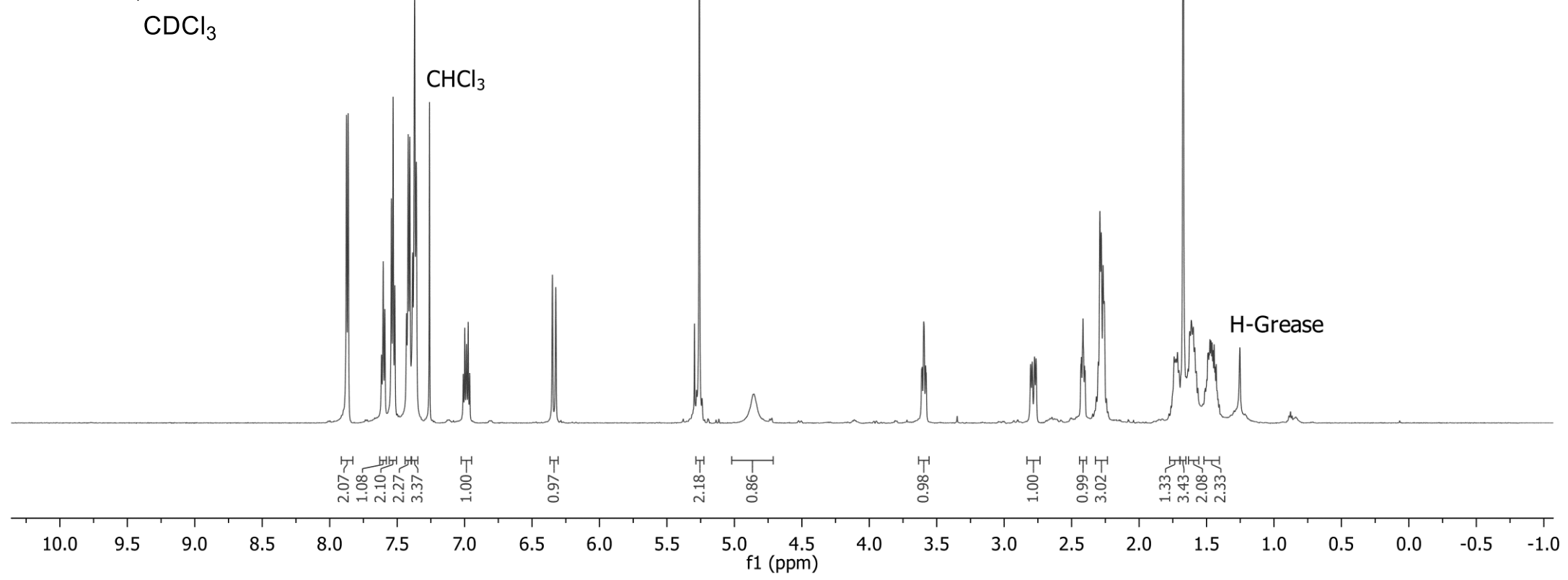




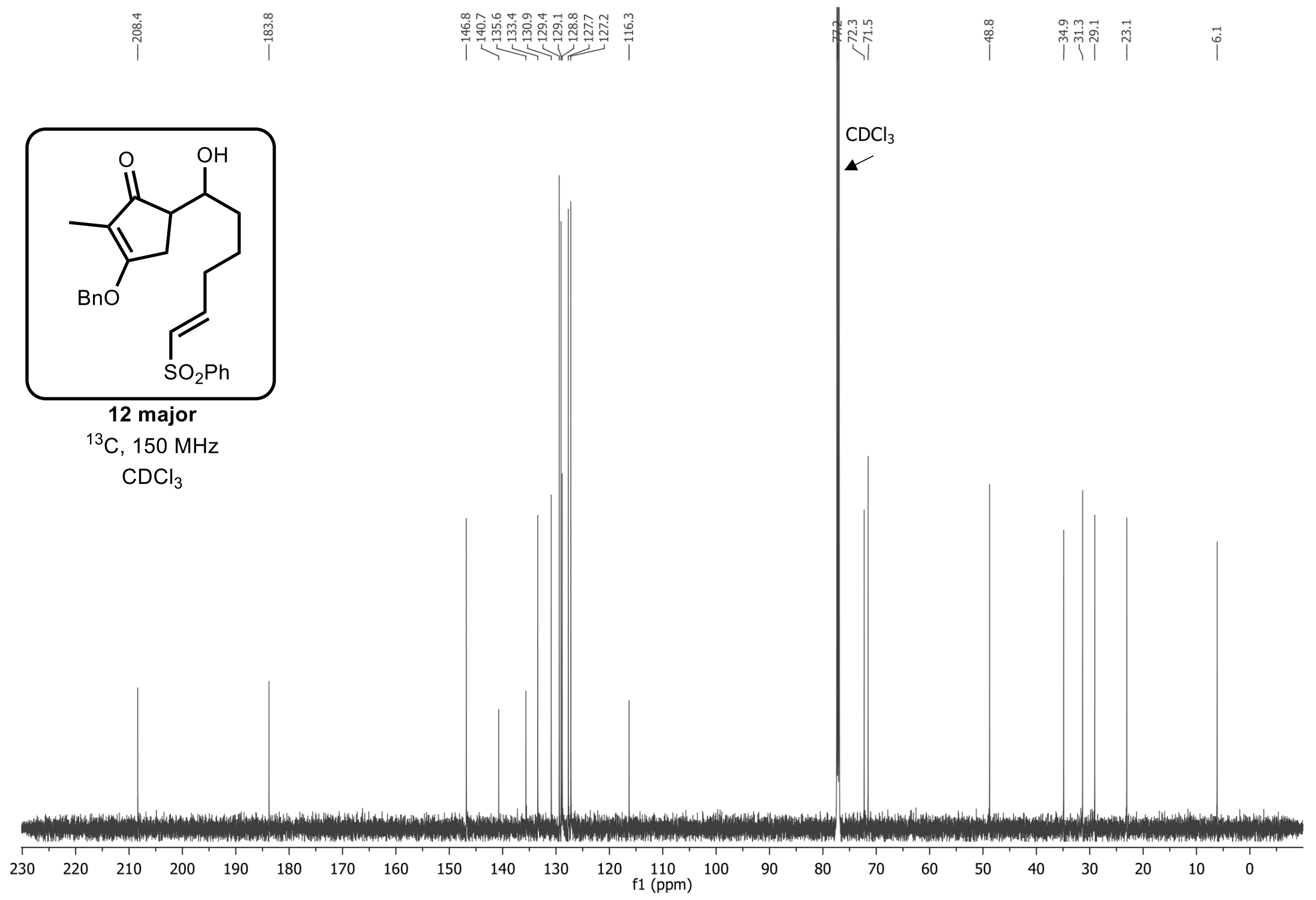




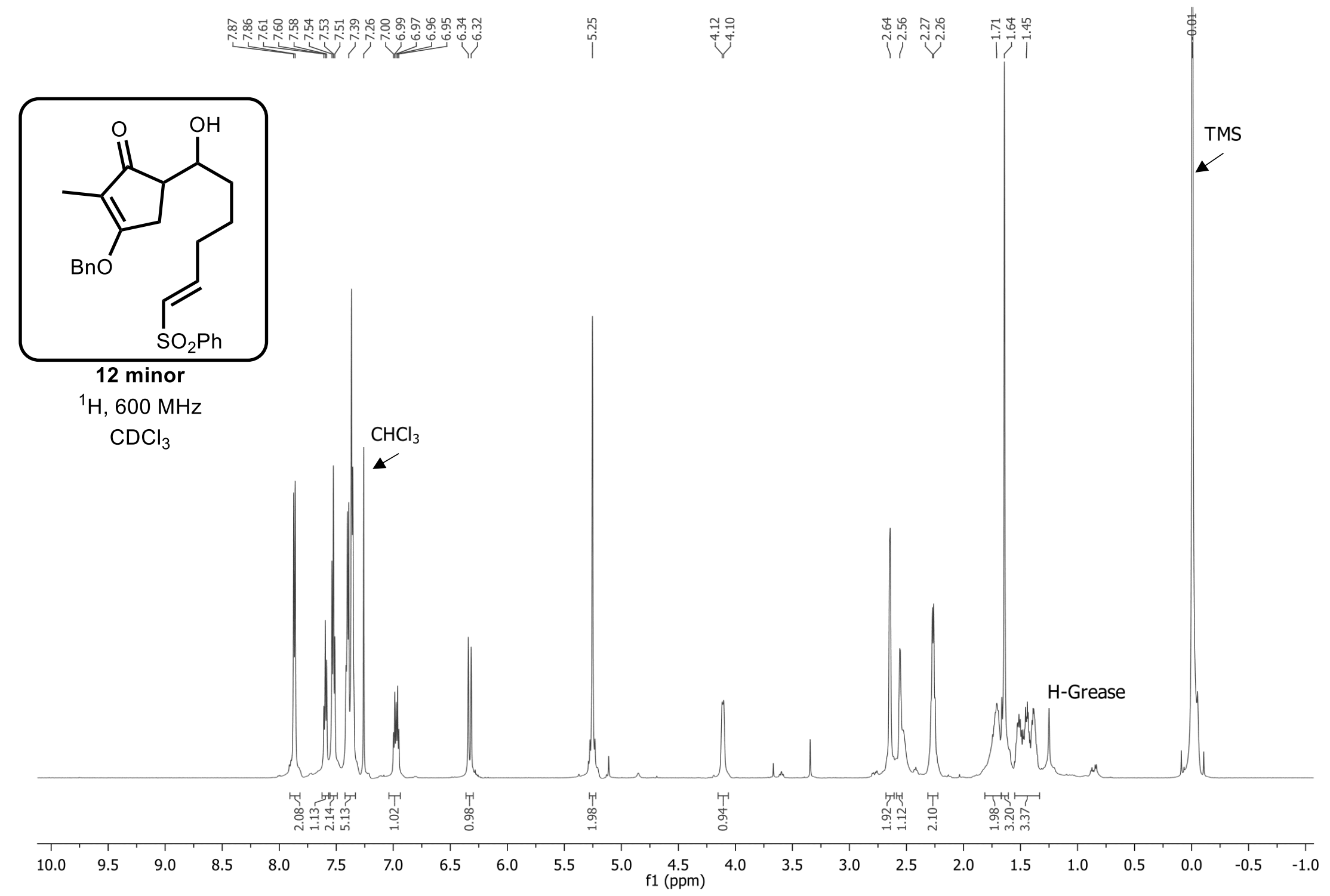




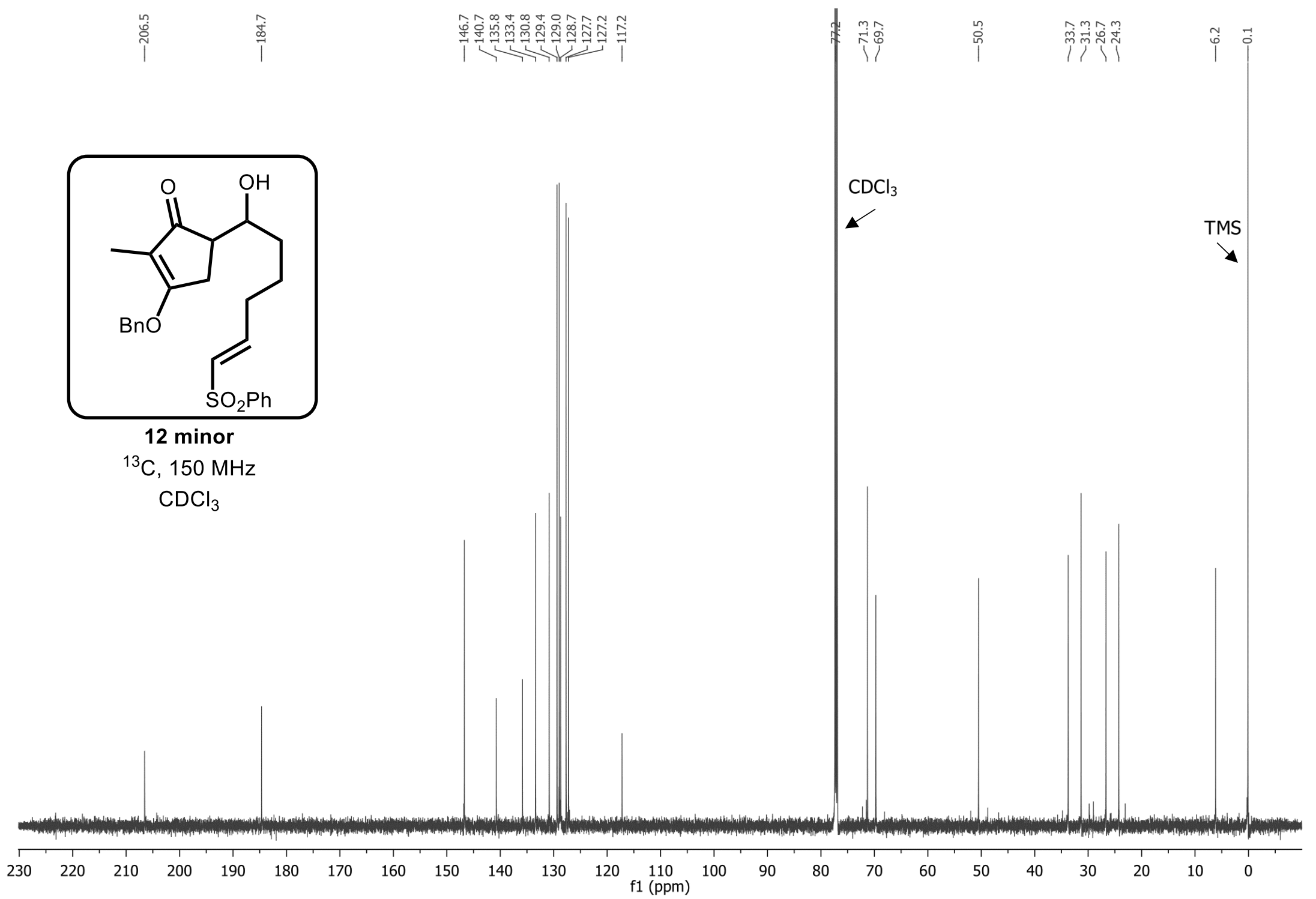




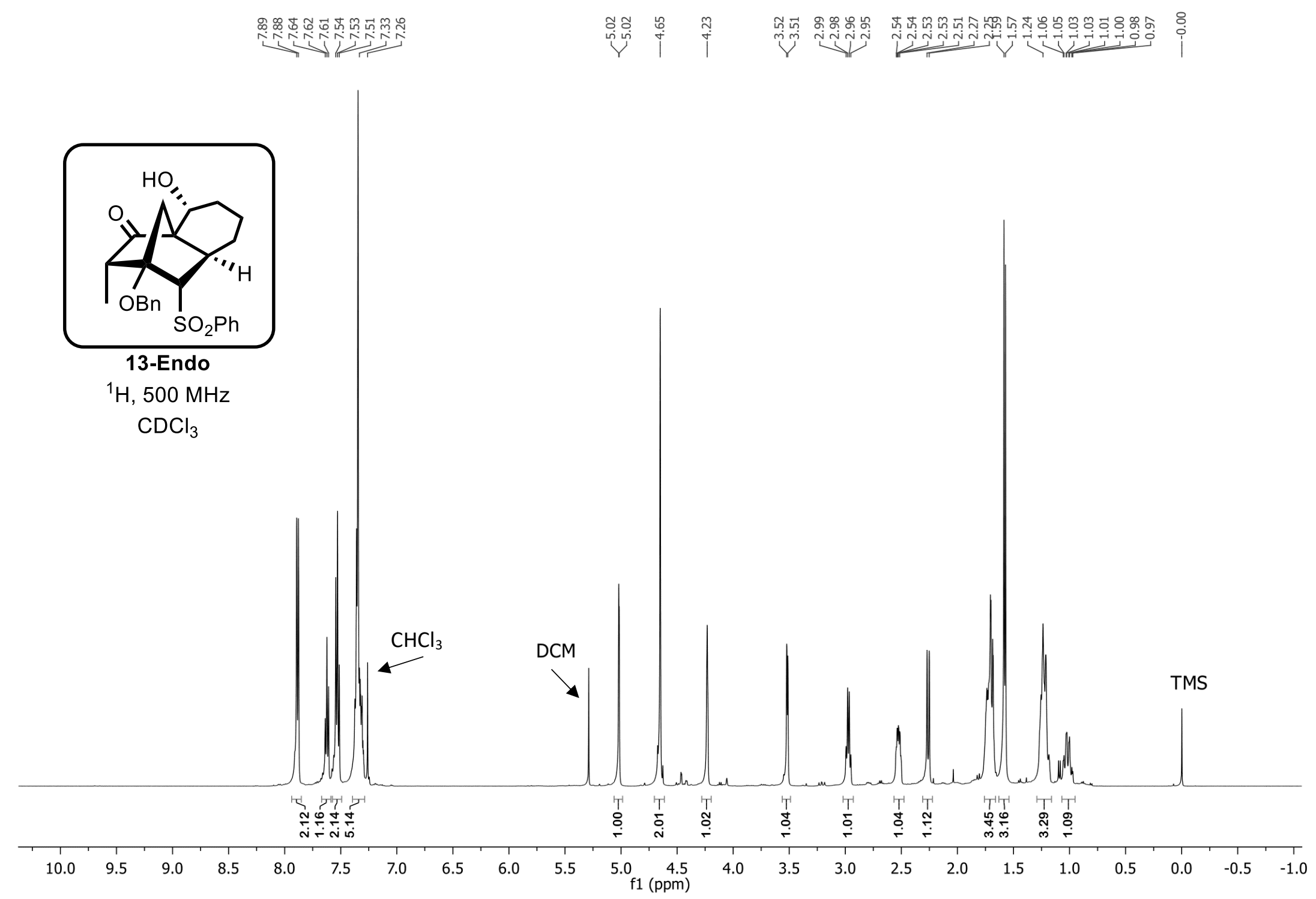




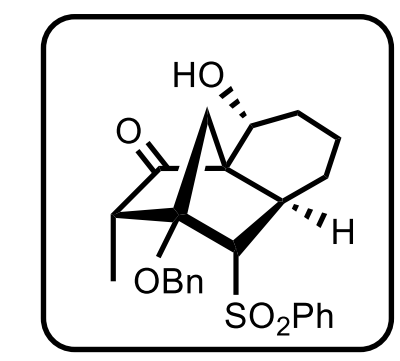

13-Endo

${ }^{13} \mathrm{C}, 125 \mathrm{MHz}$ $\mathrm{CDCl}_{3}$

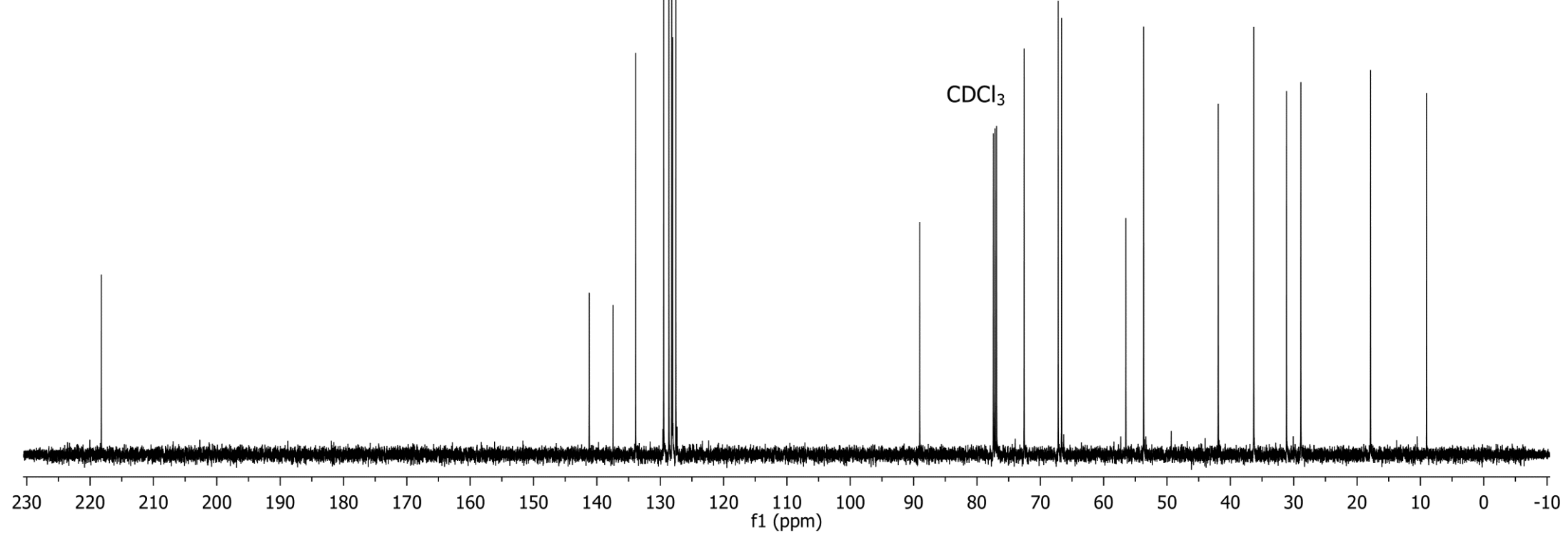




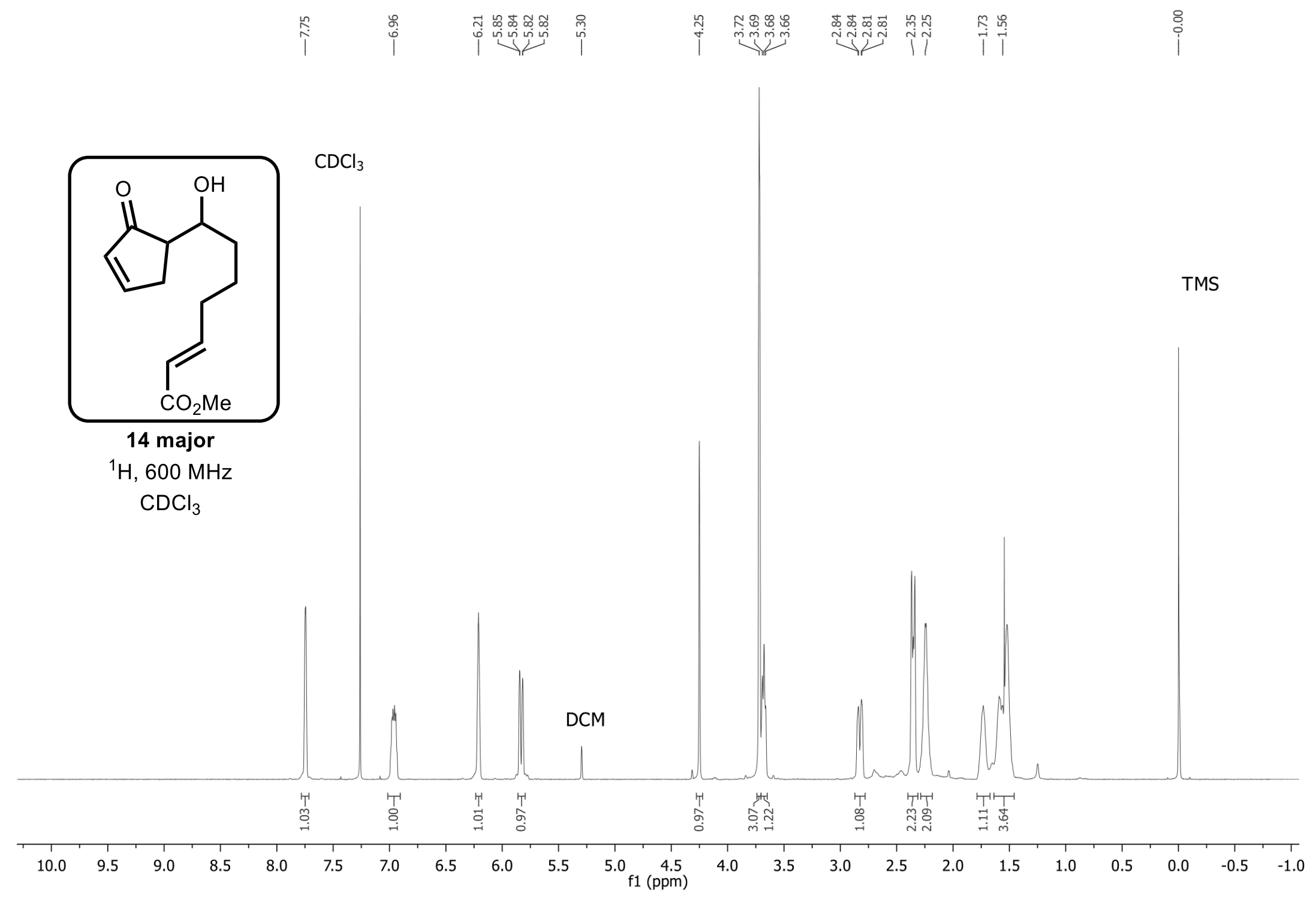




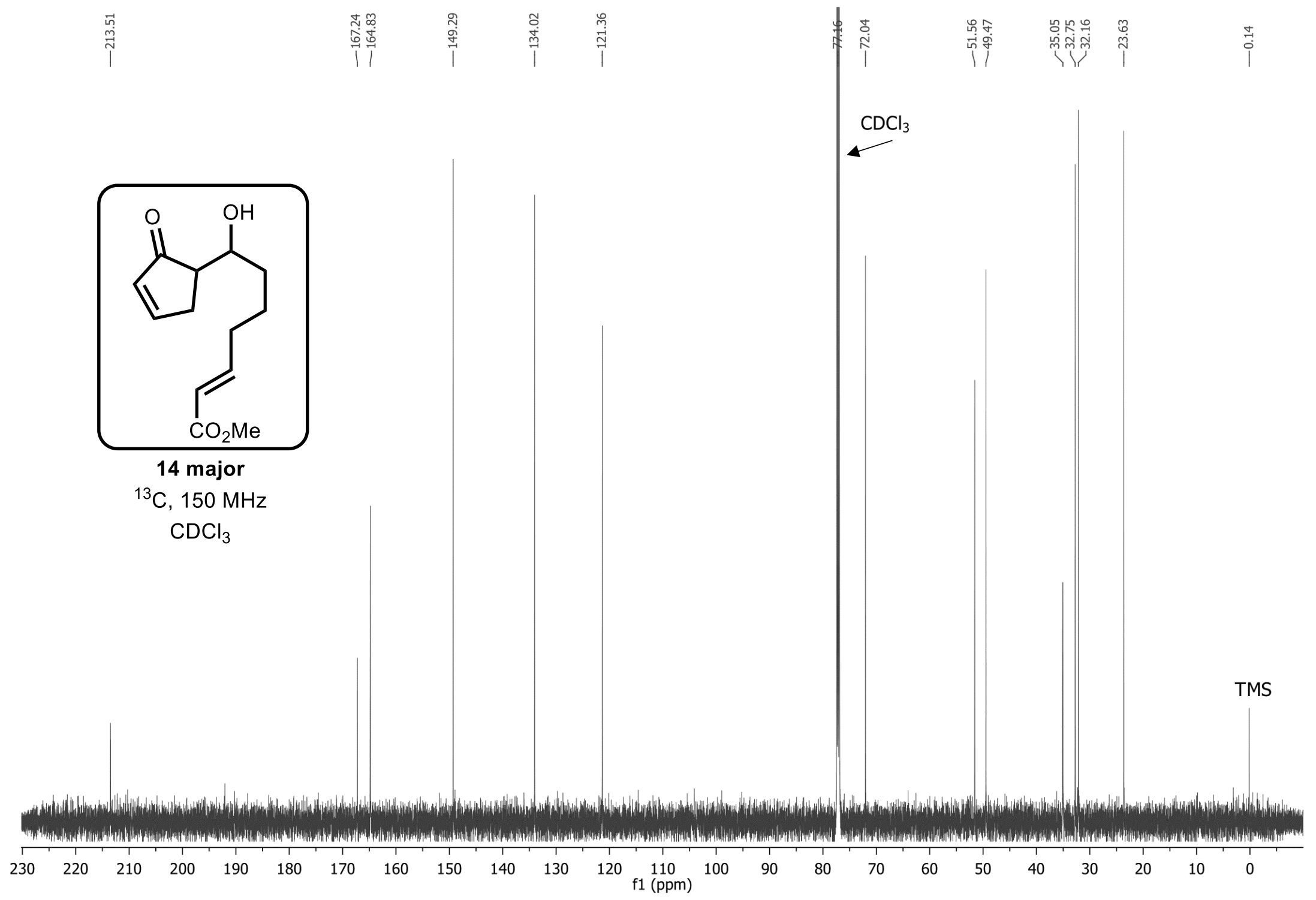




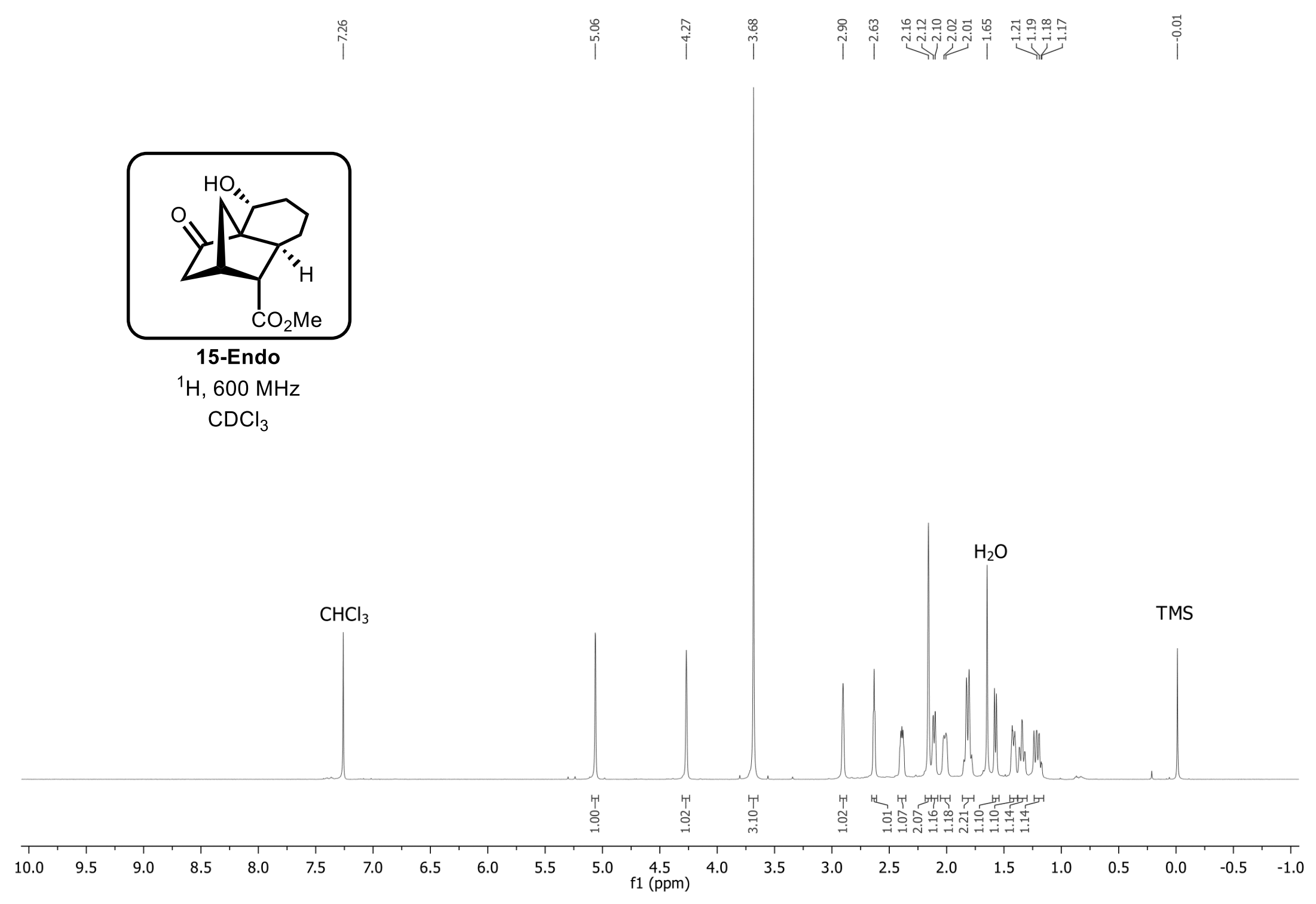




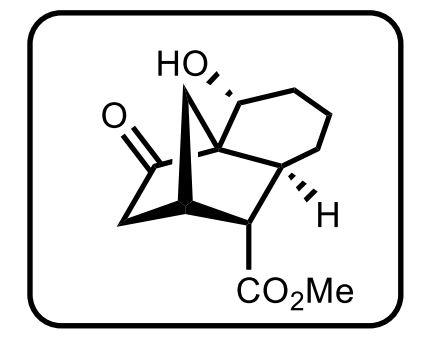

$\mathrm{CDCl}_{3}$

15-Endo

${ }^{13} \mathrm{C}, 150 \mathrm{MHz}$ $\mathrm{CDCl}_{3}$

$\checkmark$ 


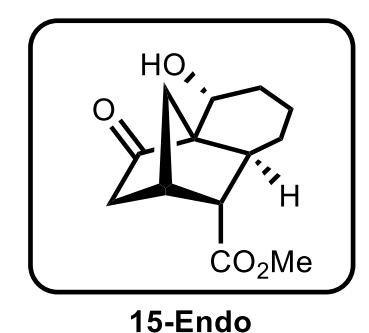

$\mathrm{H}$ COSY, $600 \mathrm{MHz}, \mathrm{CDCl}_{3}$

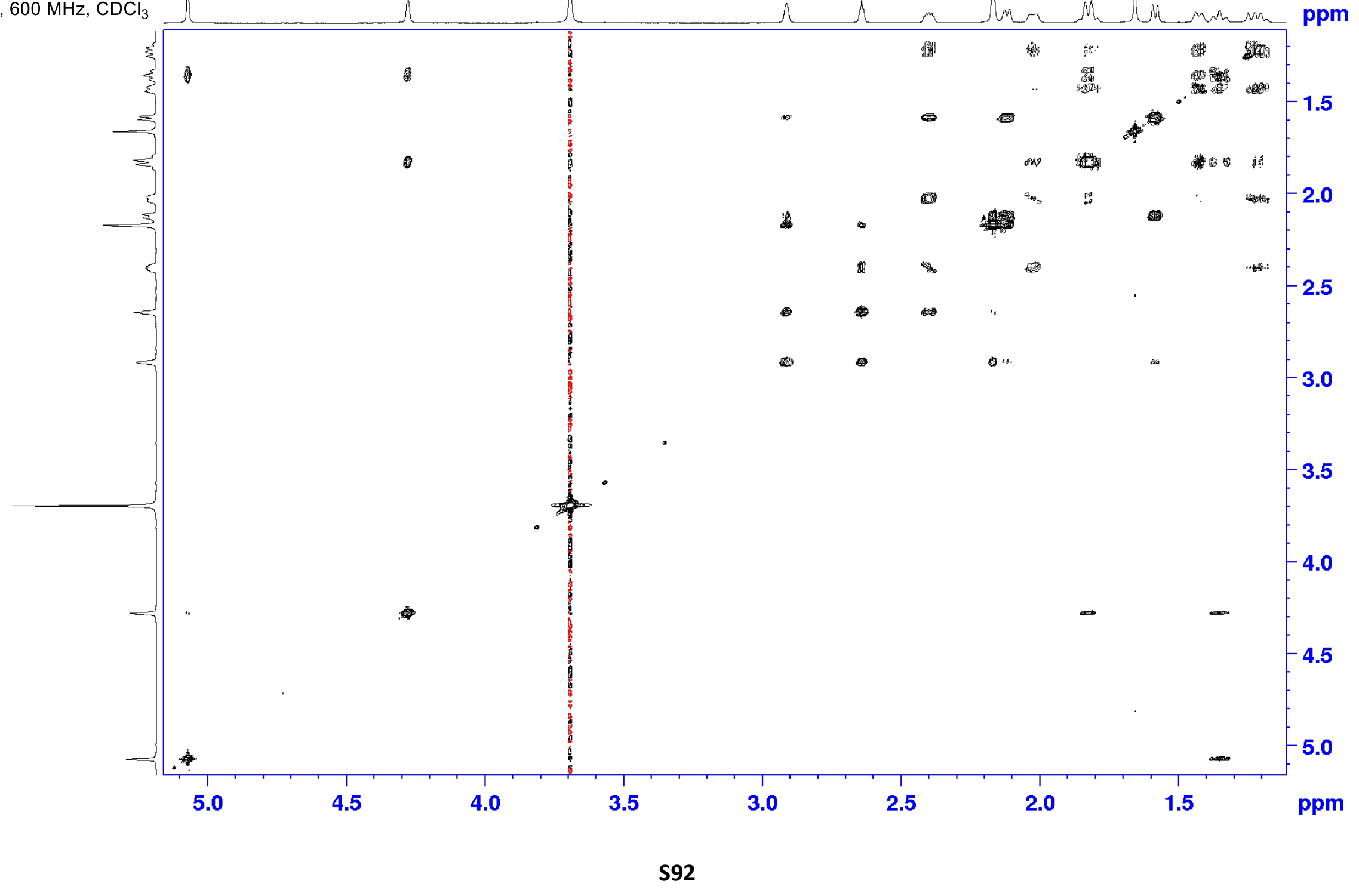




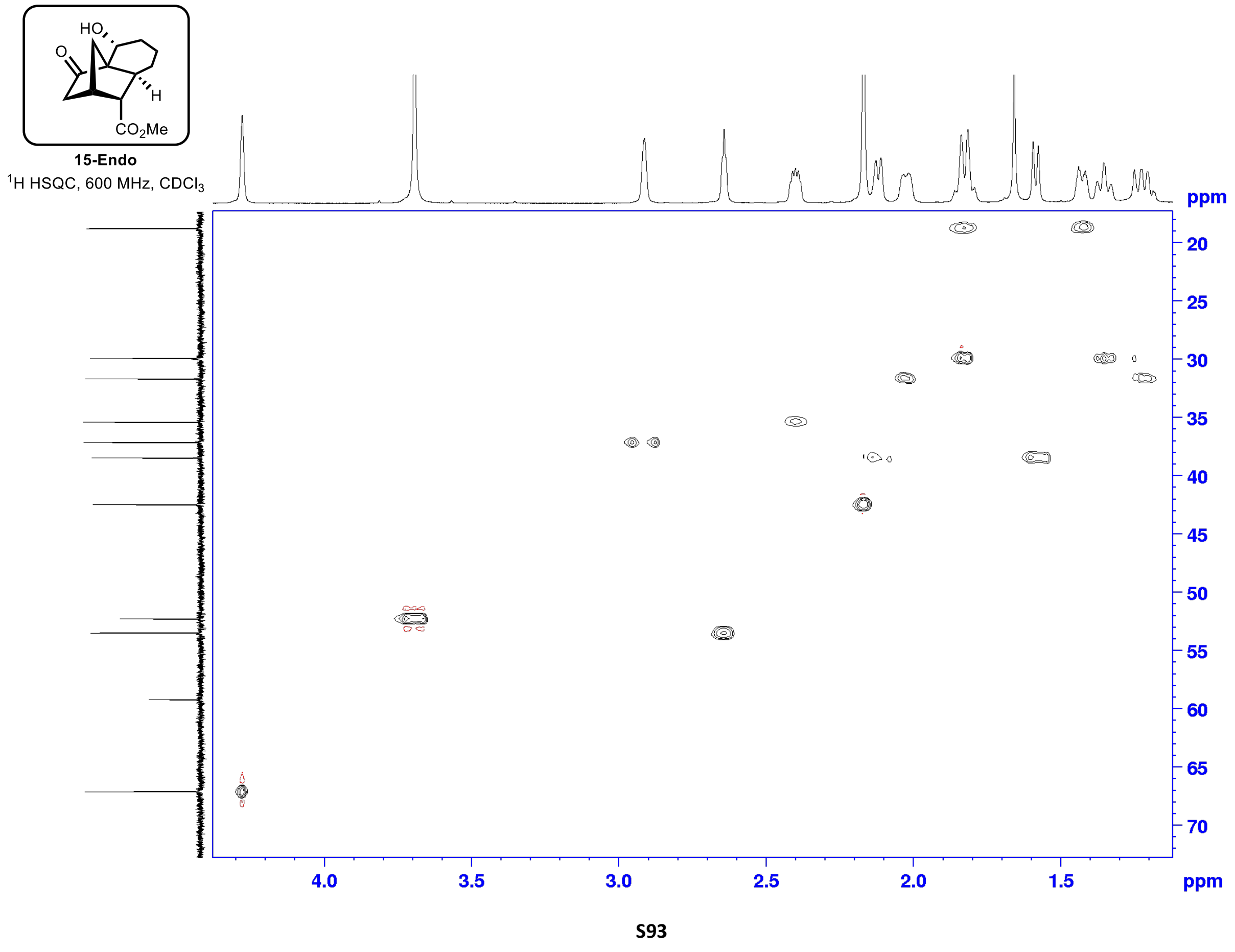




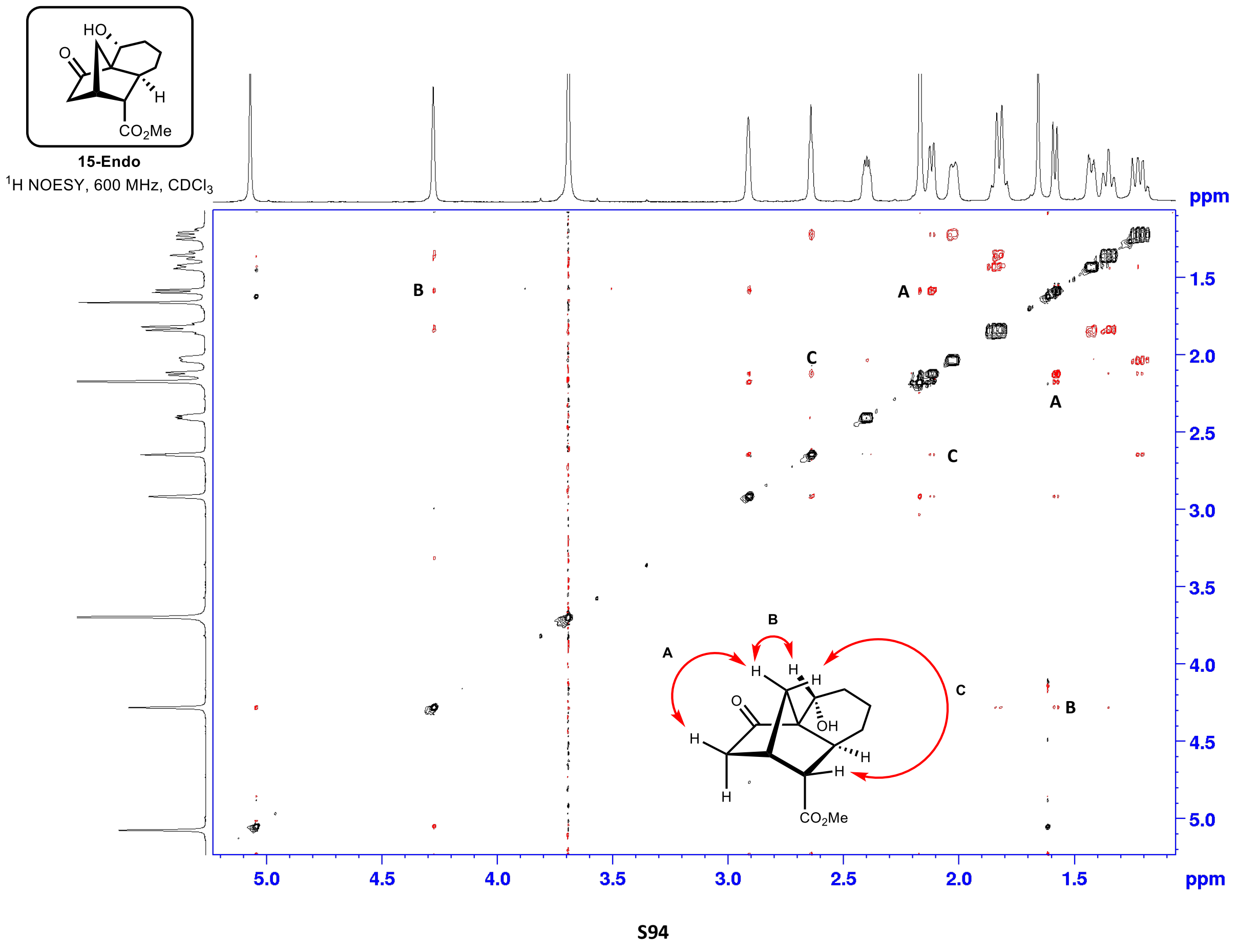




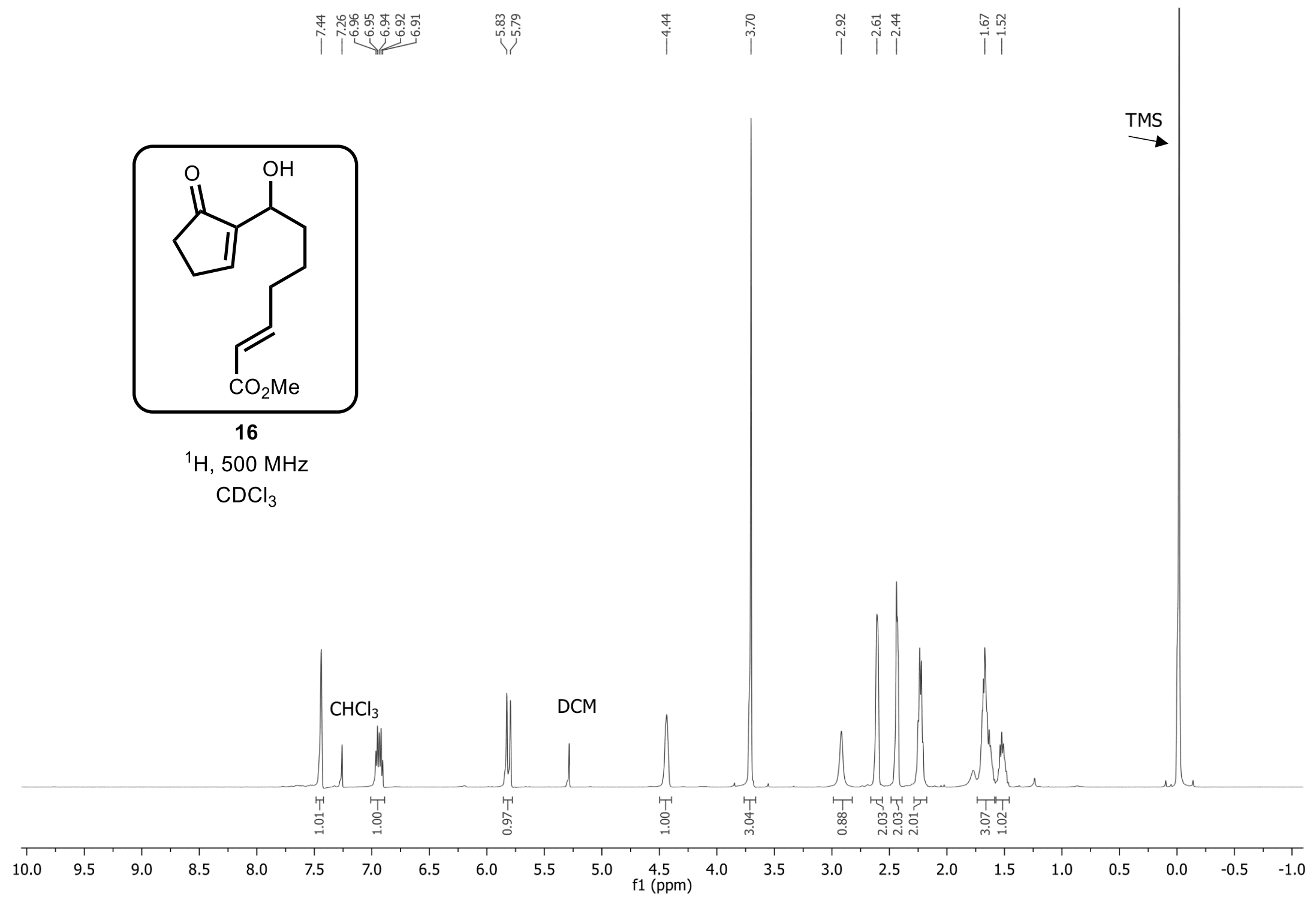




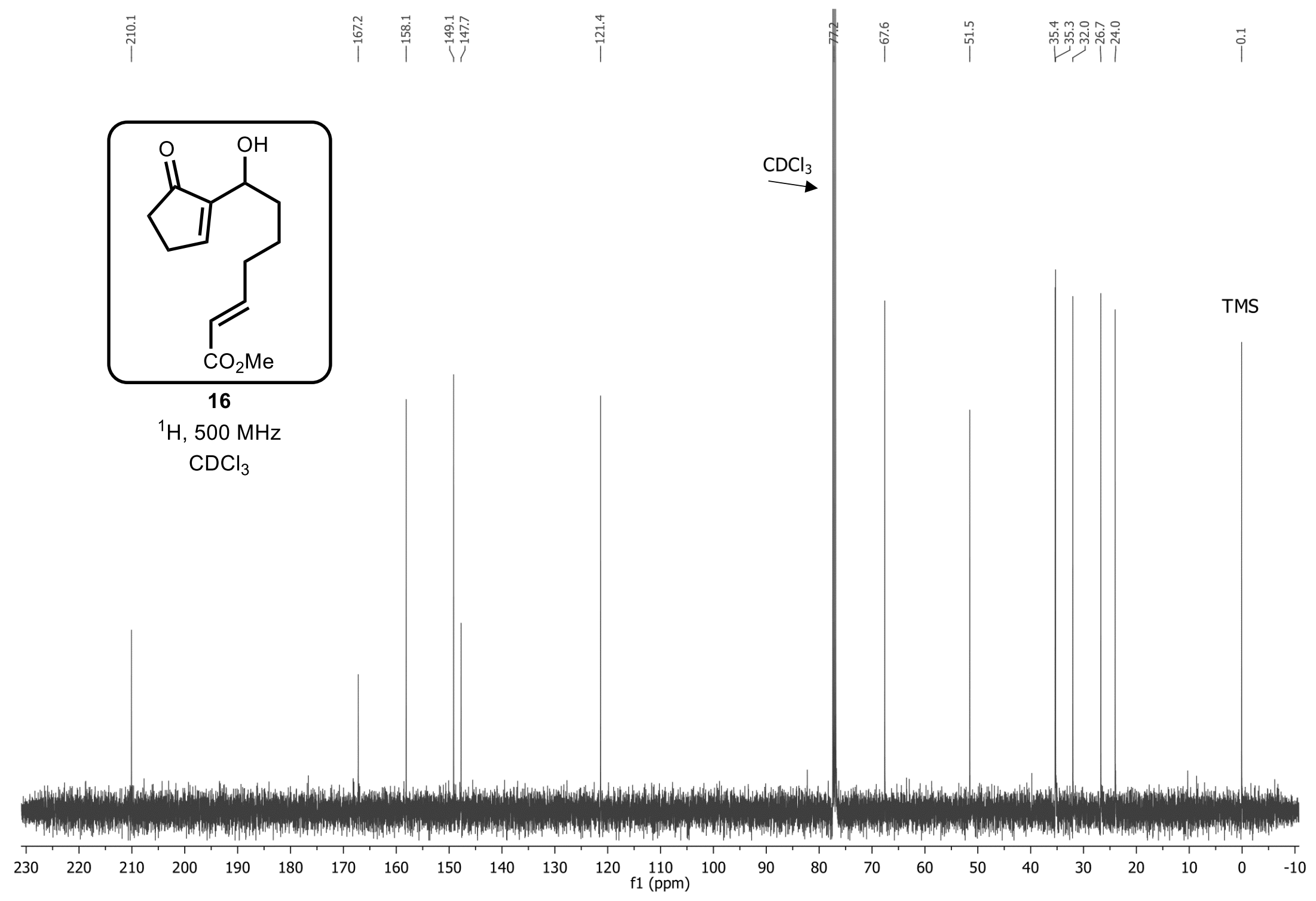




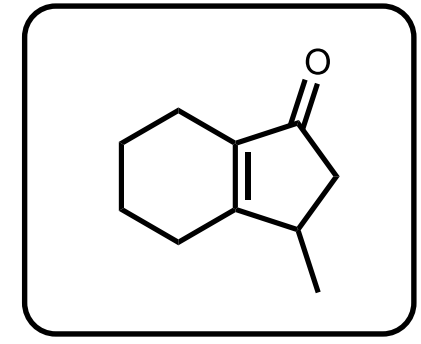

$( \pm \mathrm{S} 7)$

${ }^{1} \mathrm{H}, 500 \mathrm{MHz}$ $\mathrm{CDCl}_{3}$

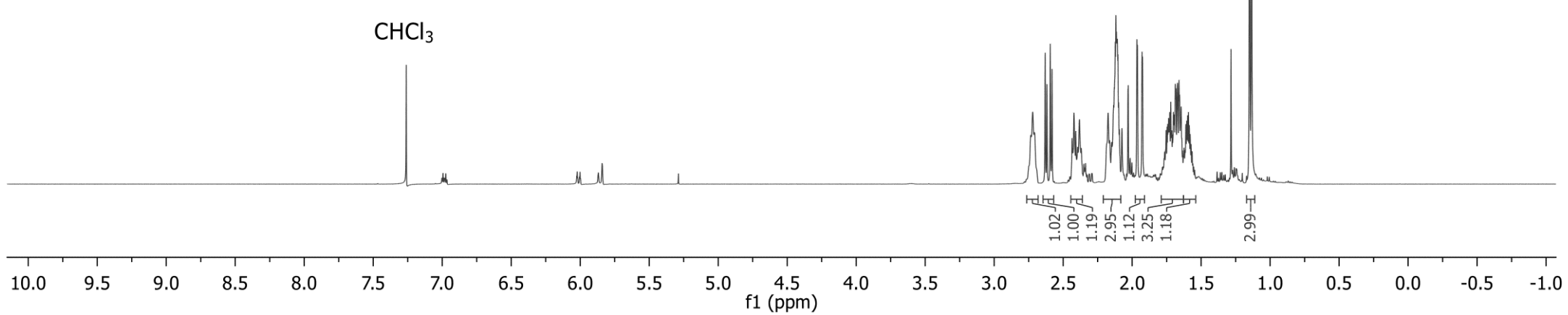




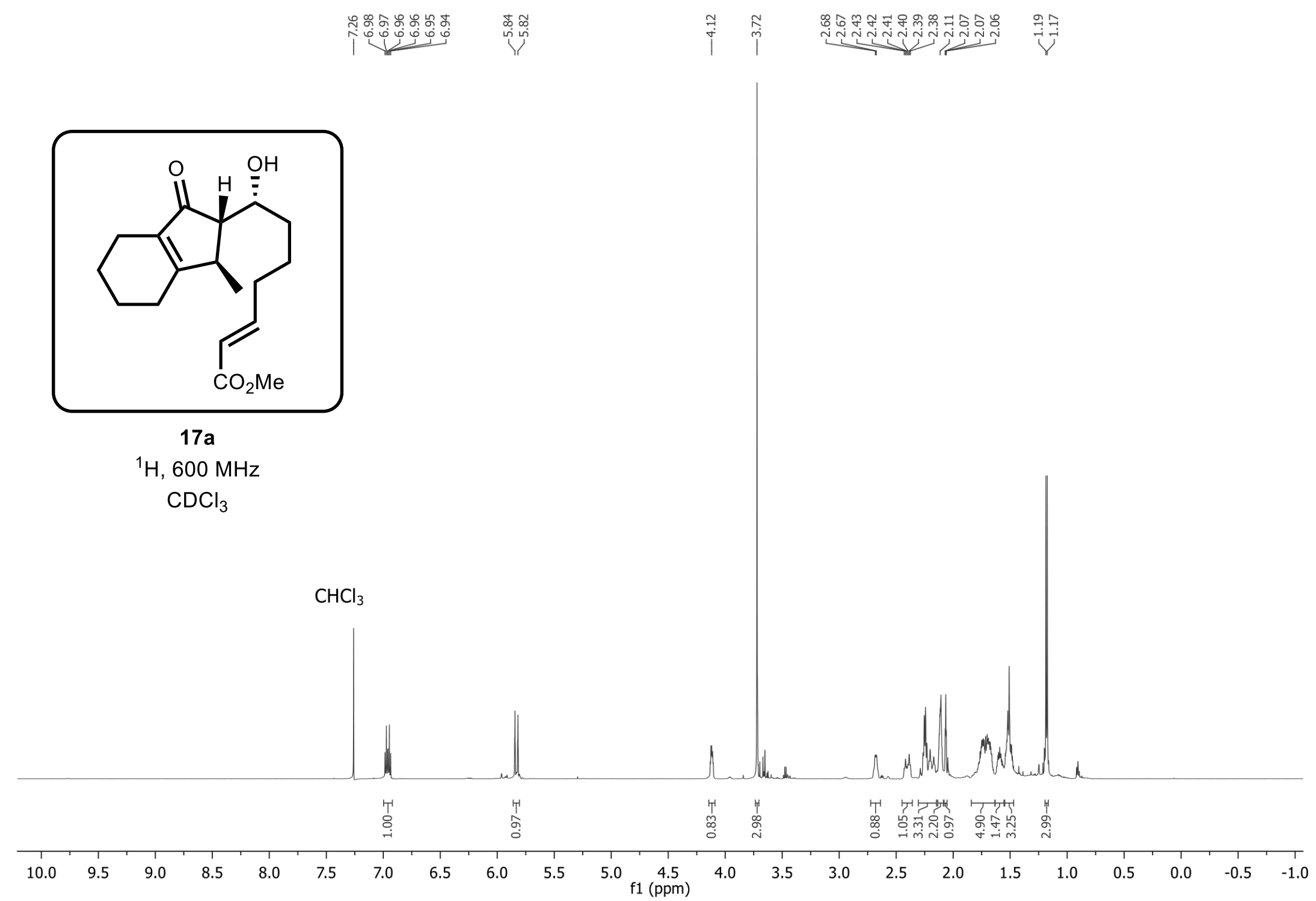




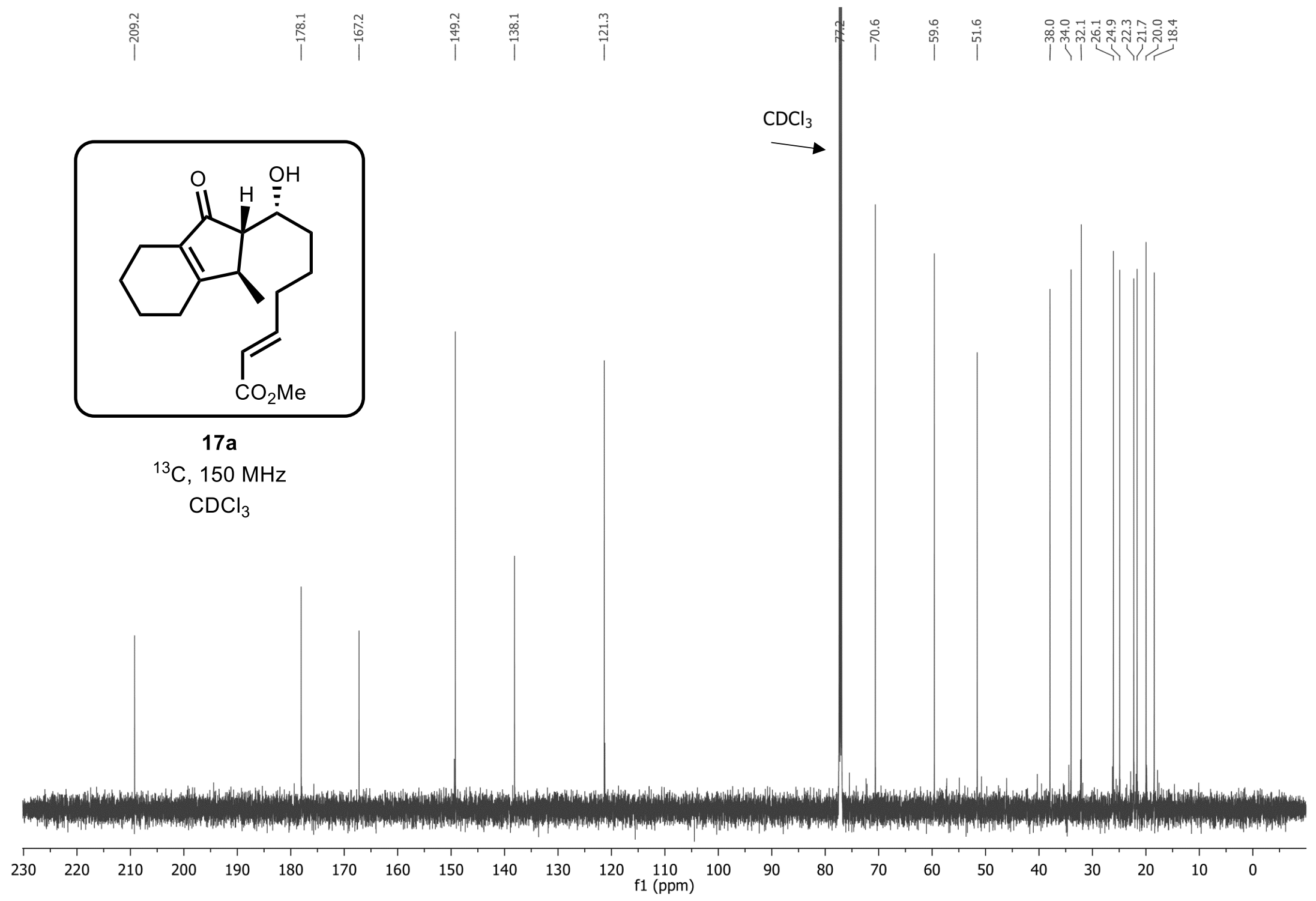




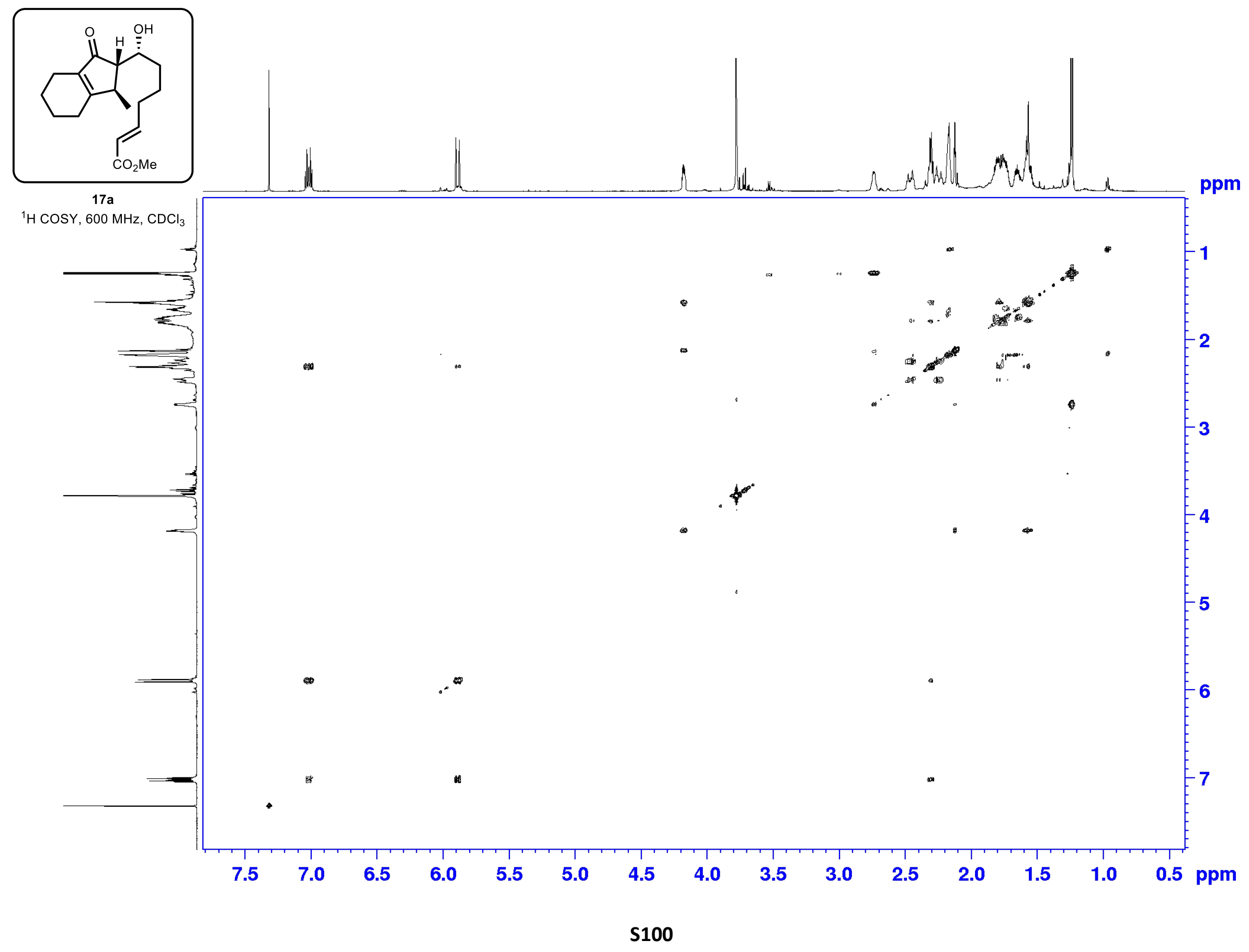




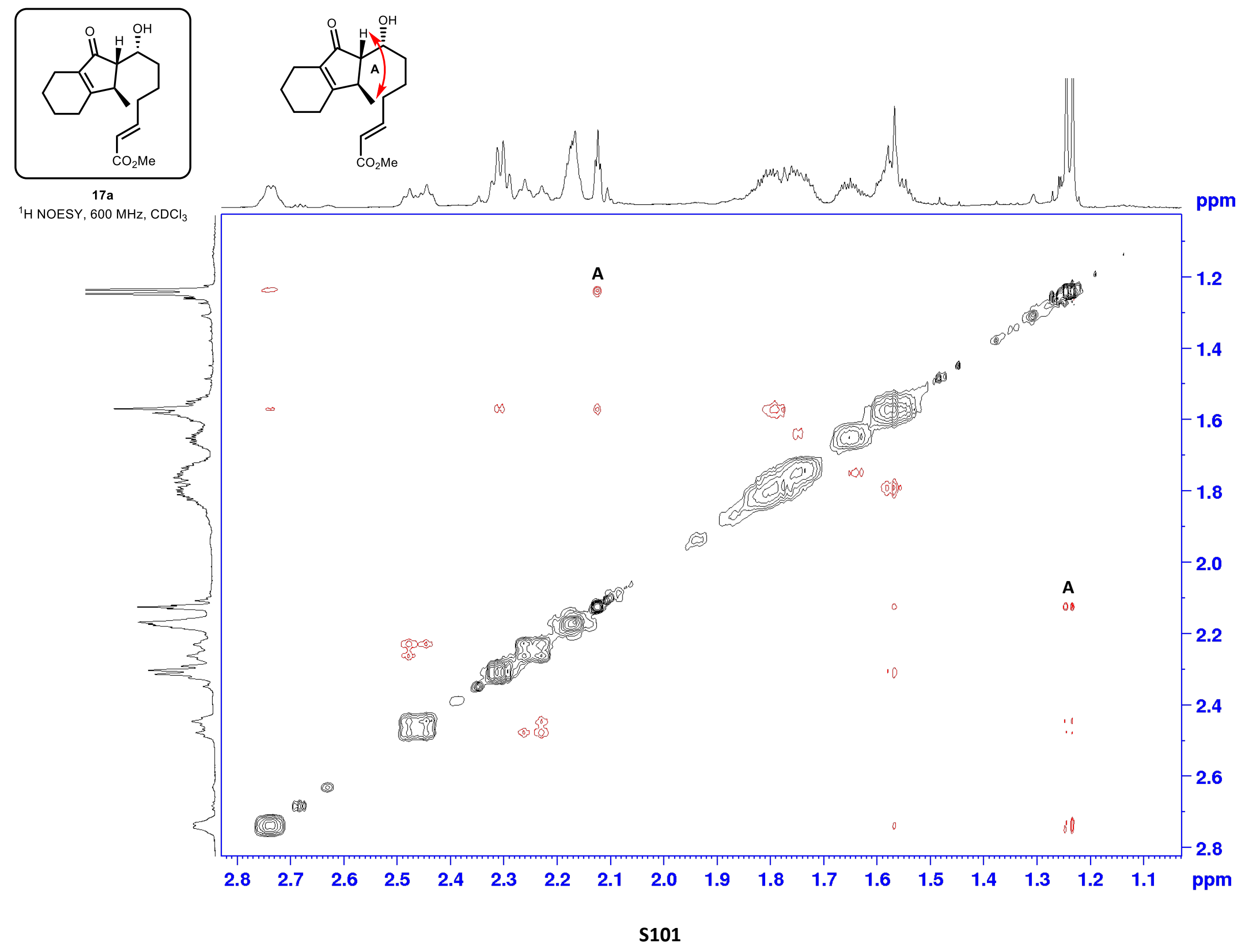




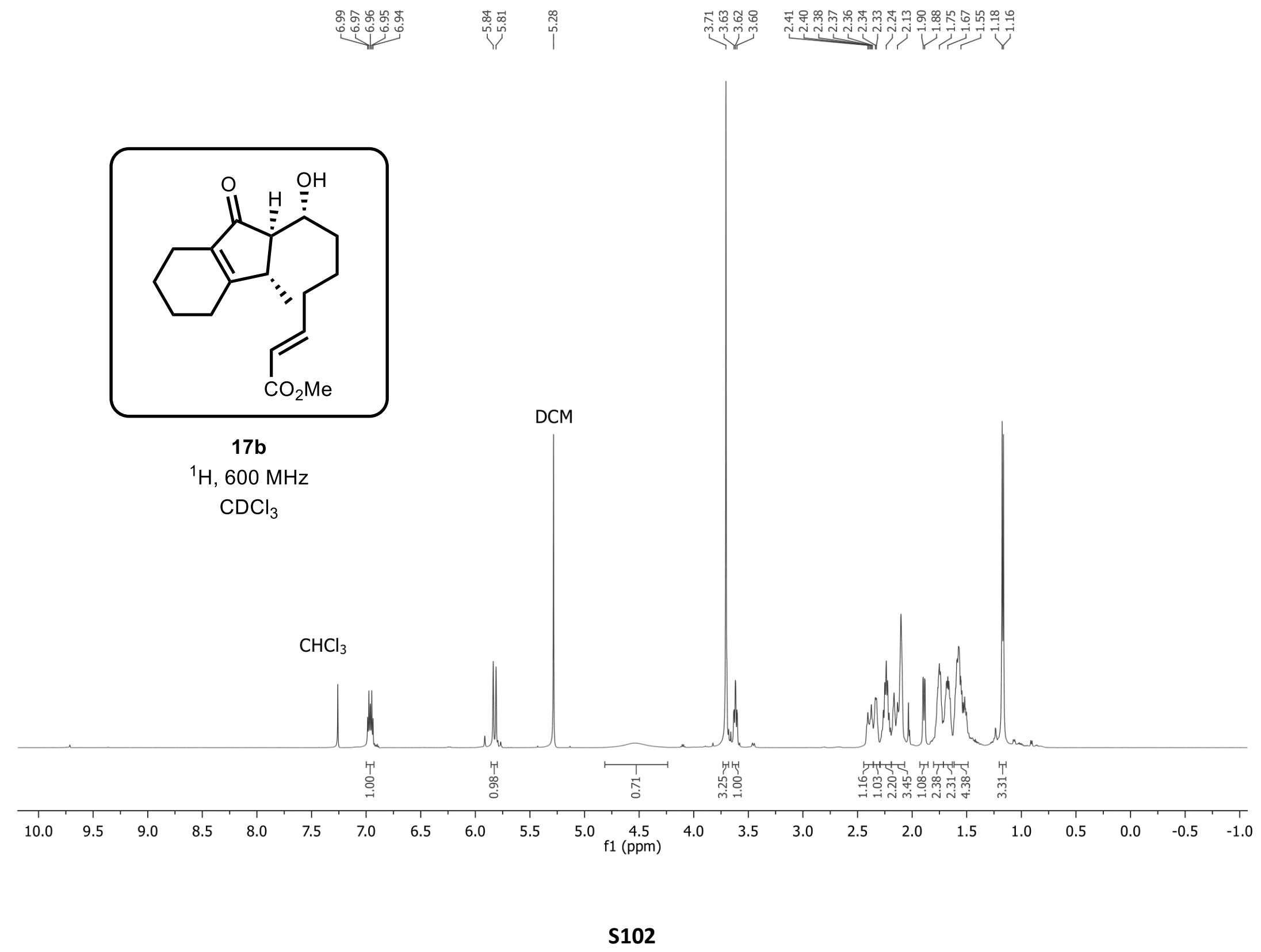




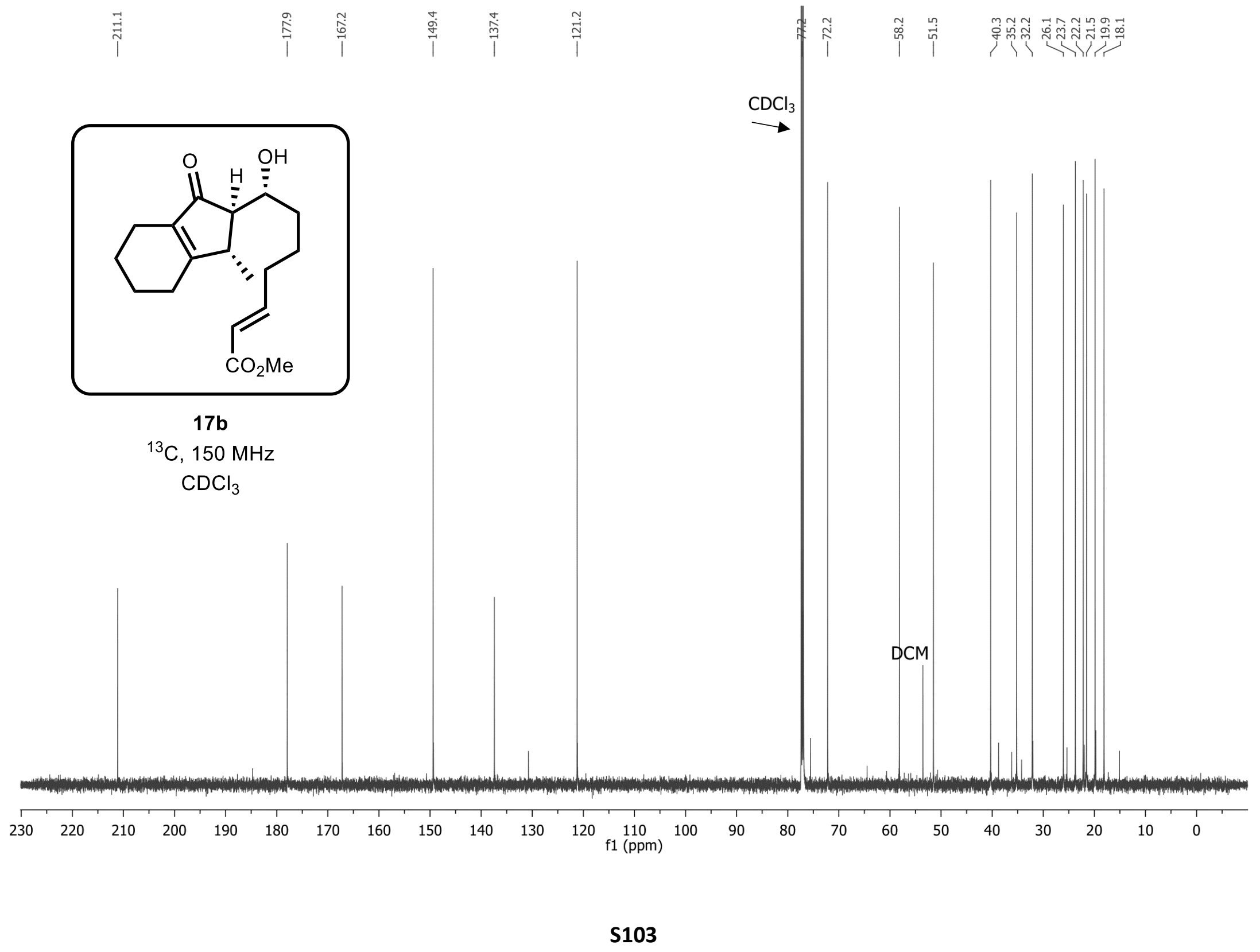




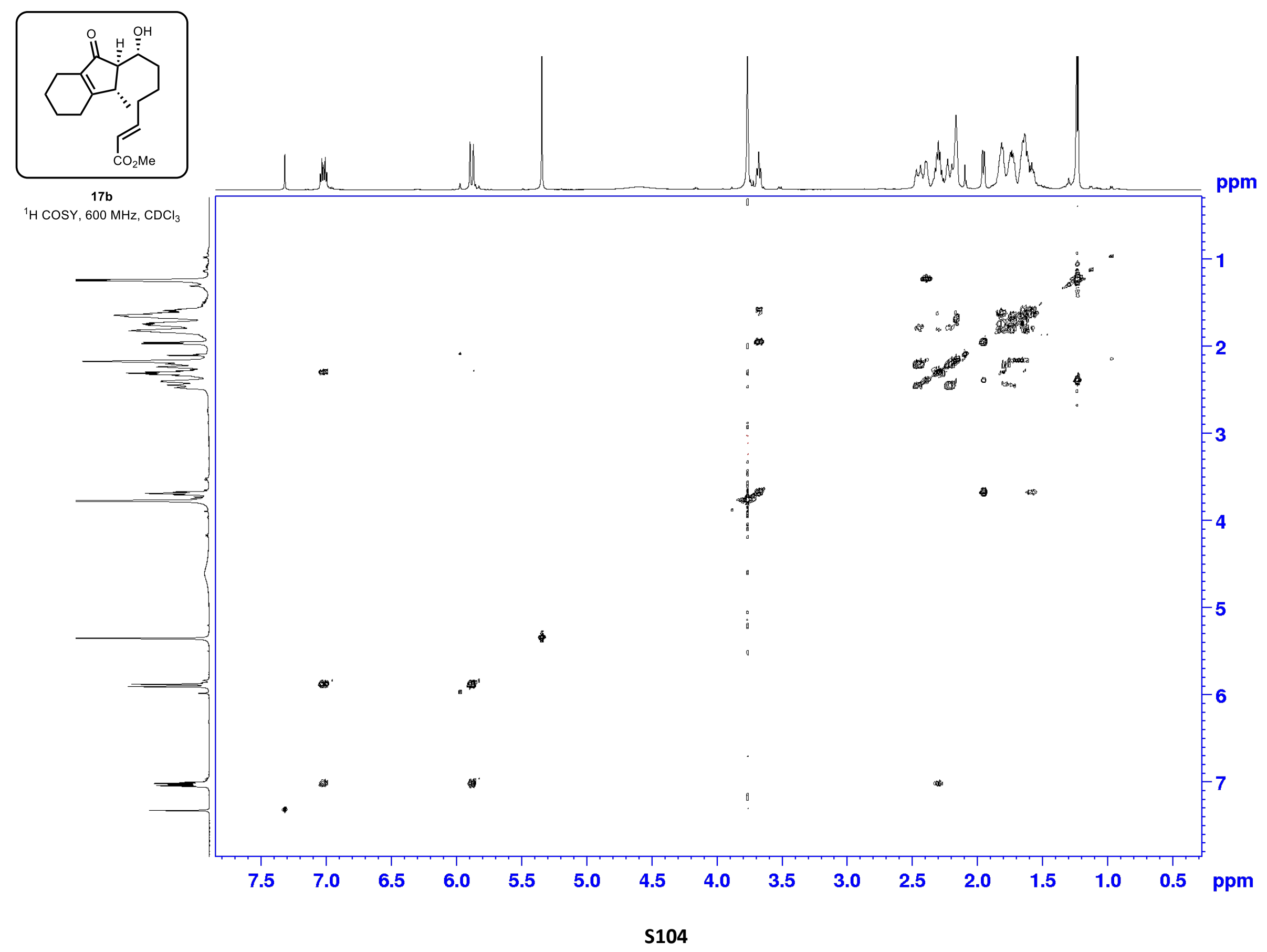




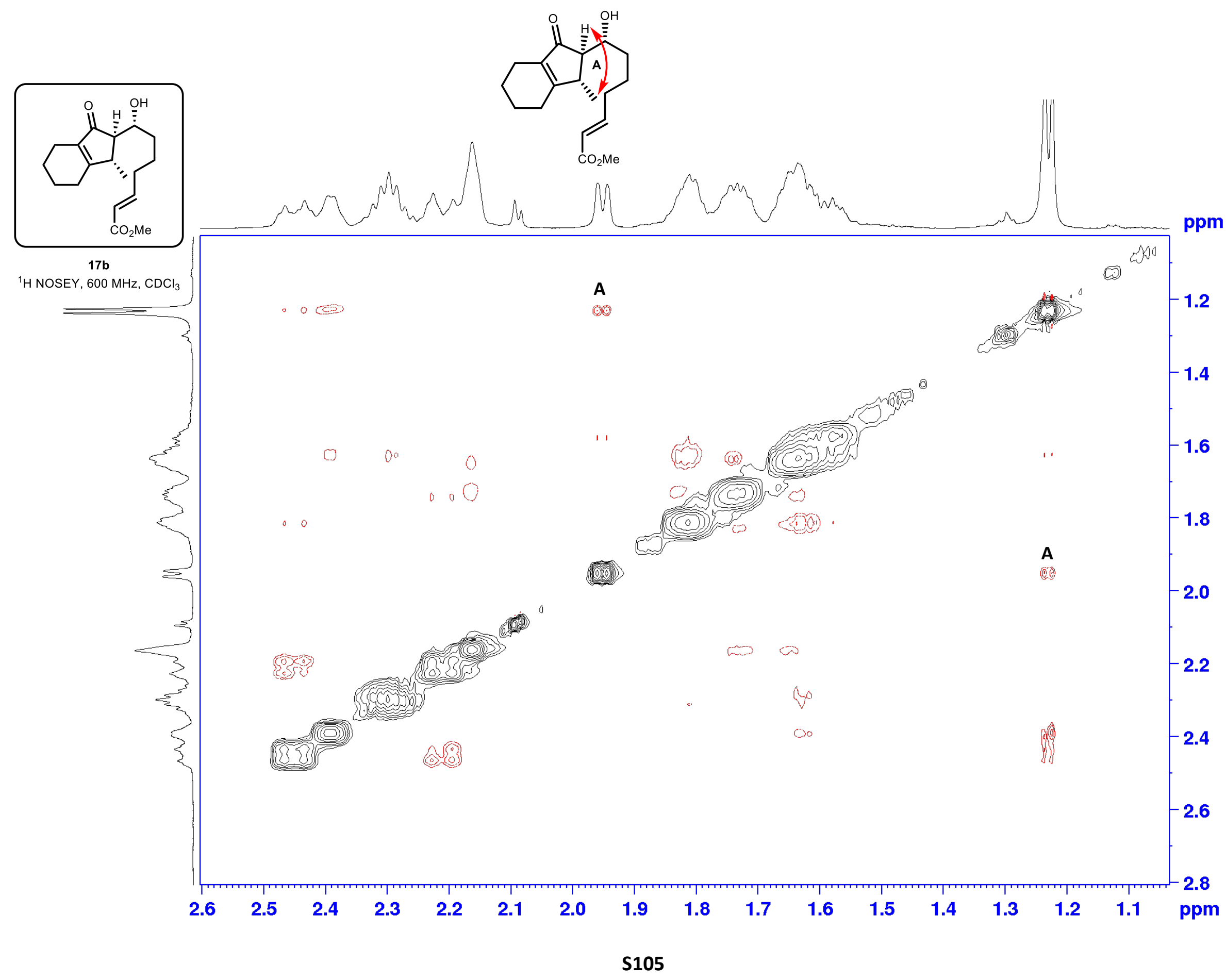




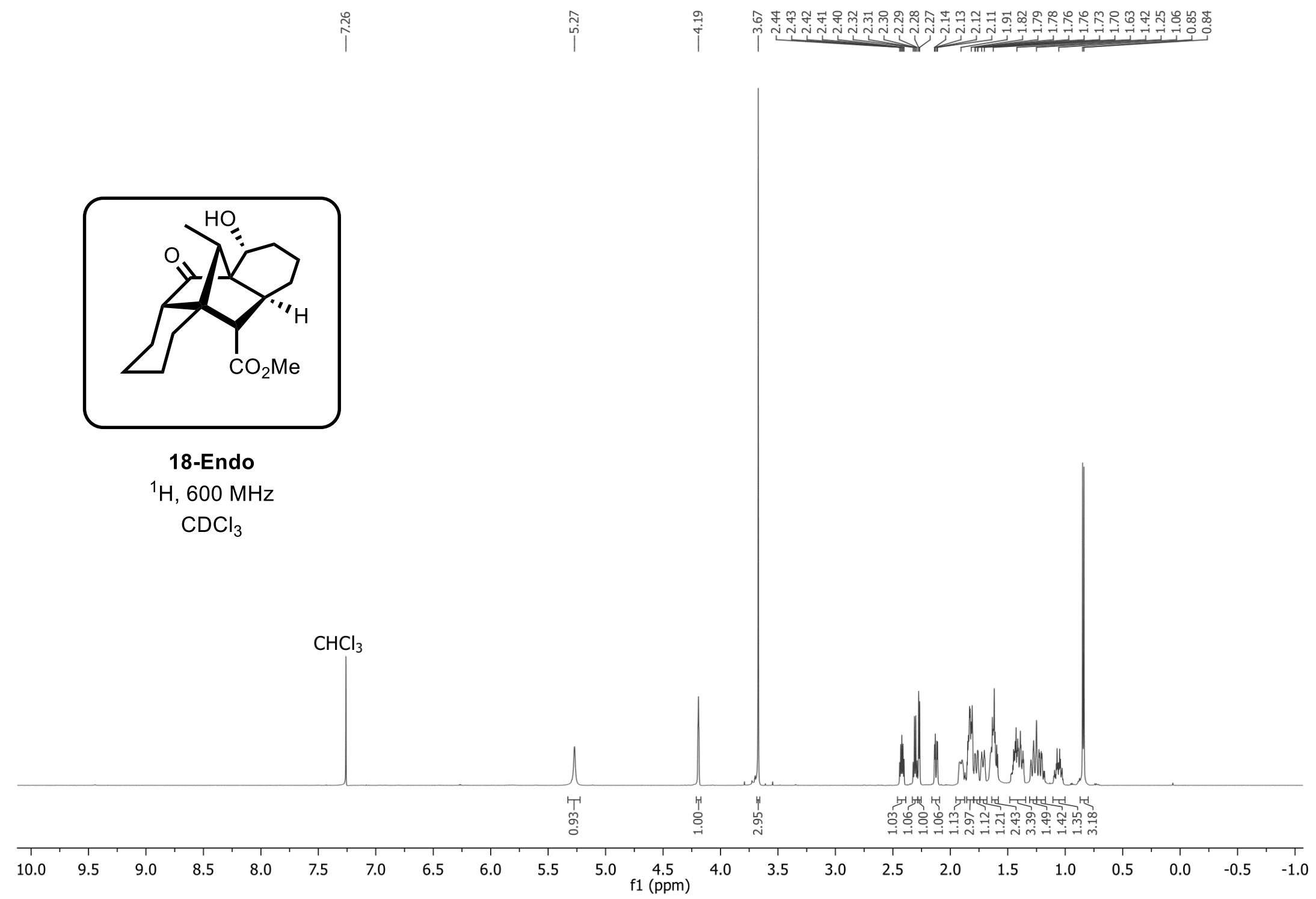




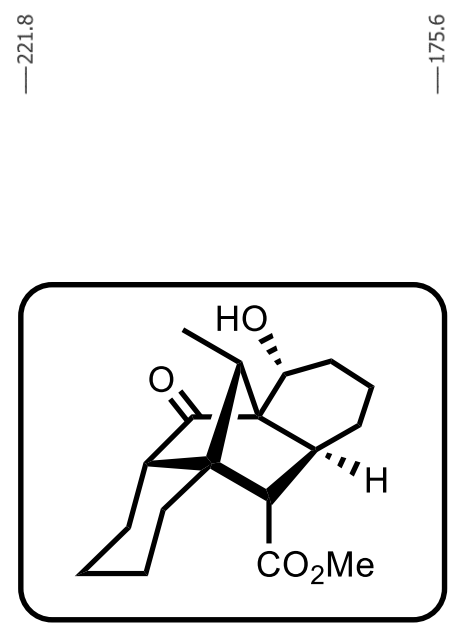

18-Endo

${ }^{13} \mathrm{C}, 150 \mathrm{MHz}$

$\mathrm{CDCl}_{3}$

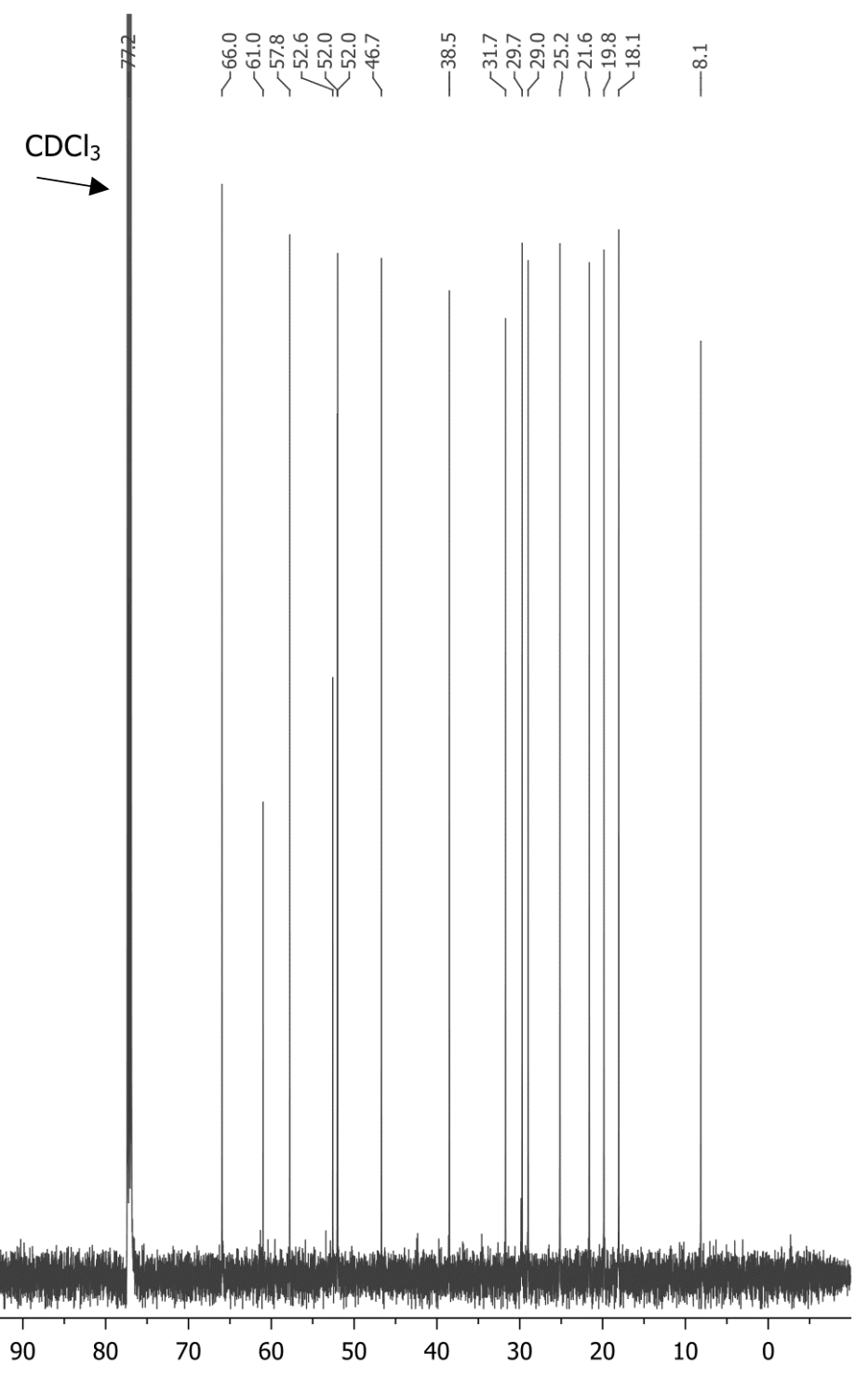




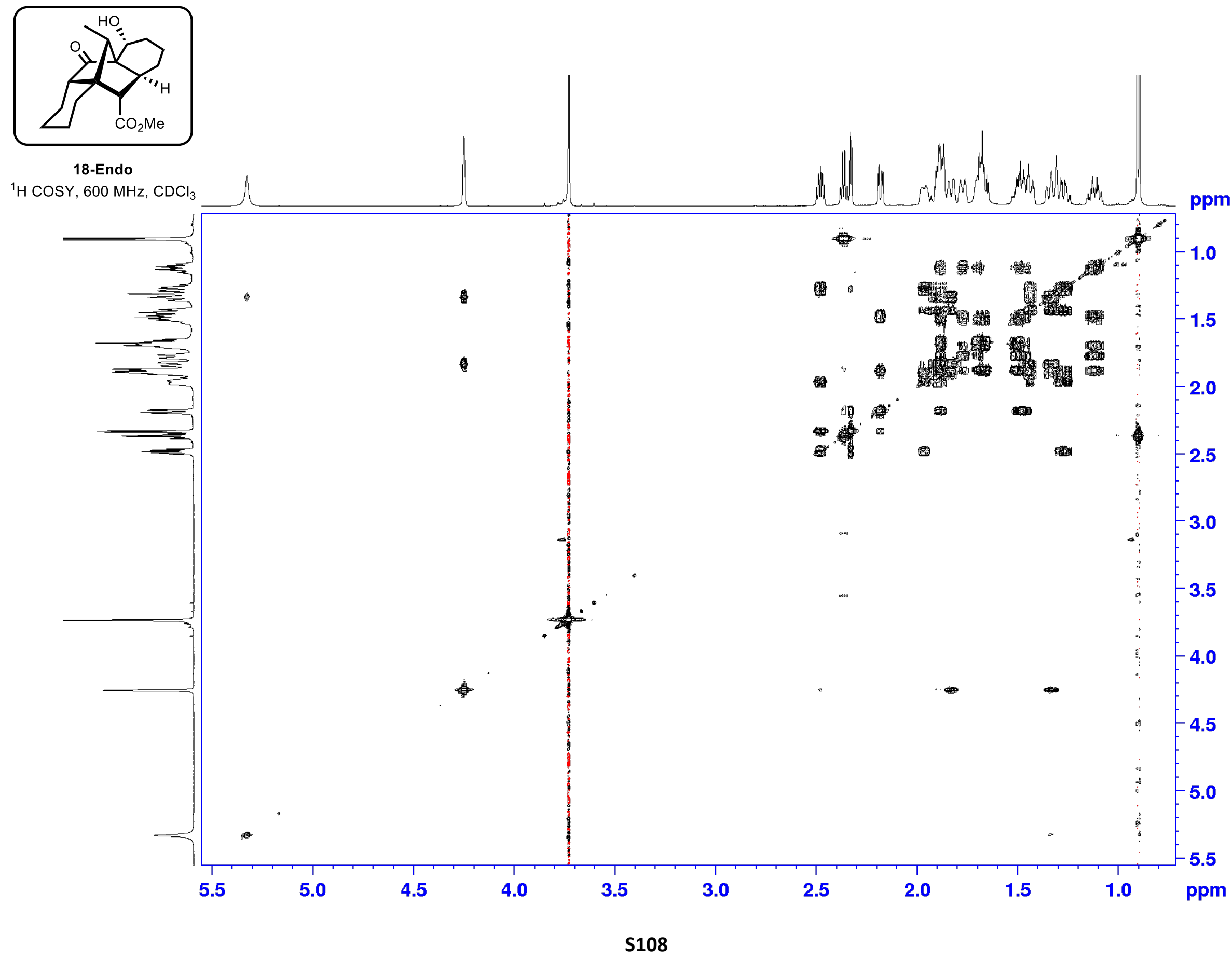




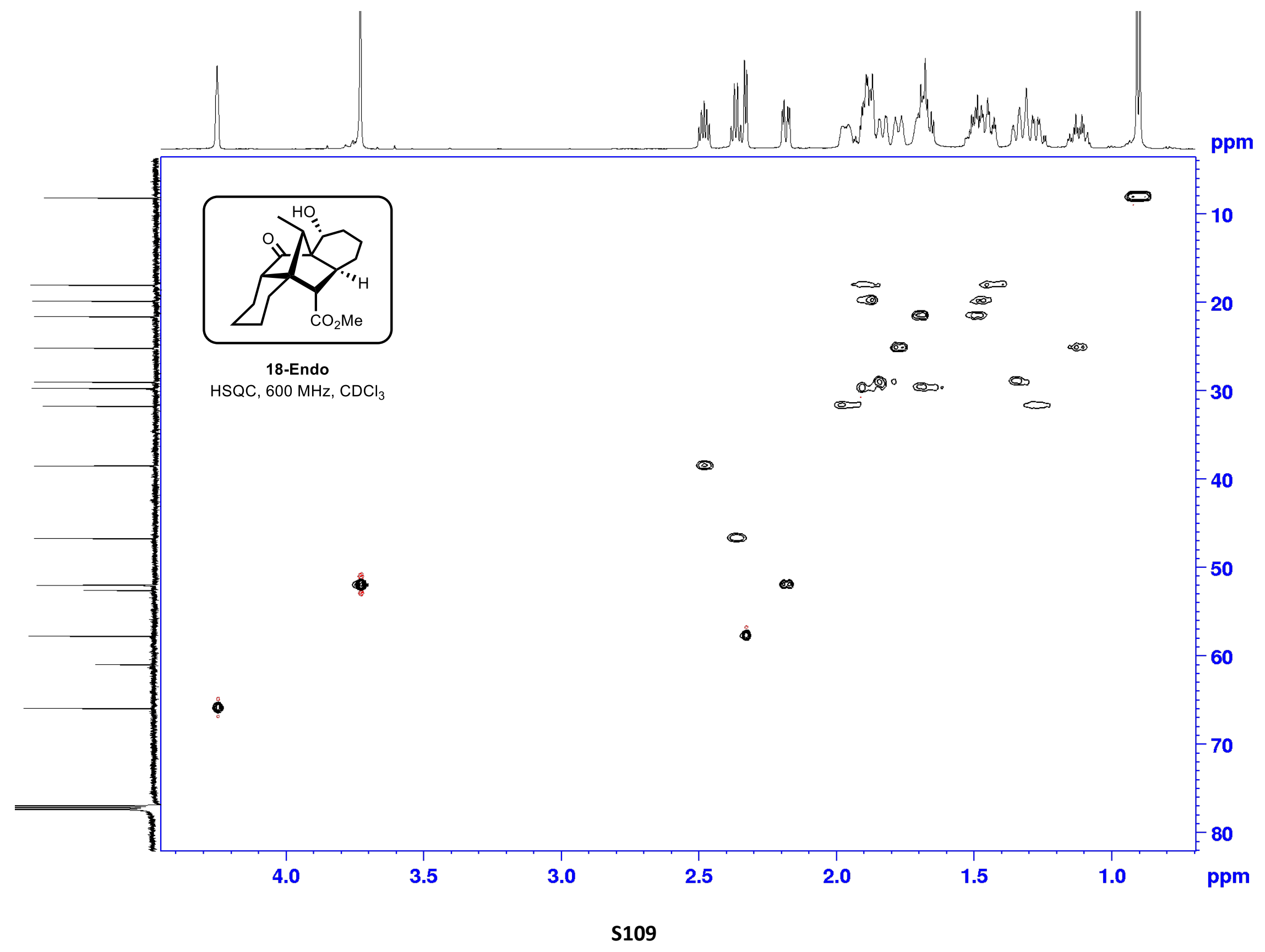




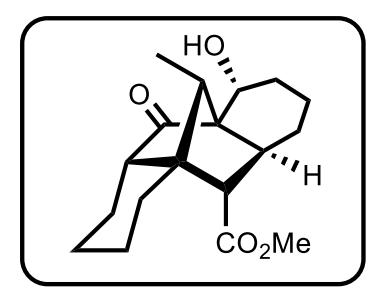

18-Endo

$\mathrm{HMBC}, 600 \mathrm{MHz}, \mathrm{CDCl}_{3}$

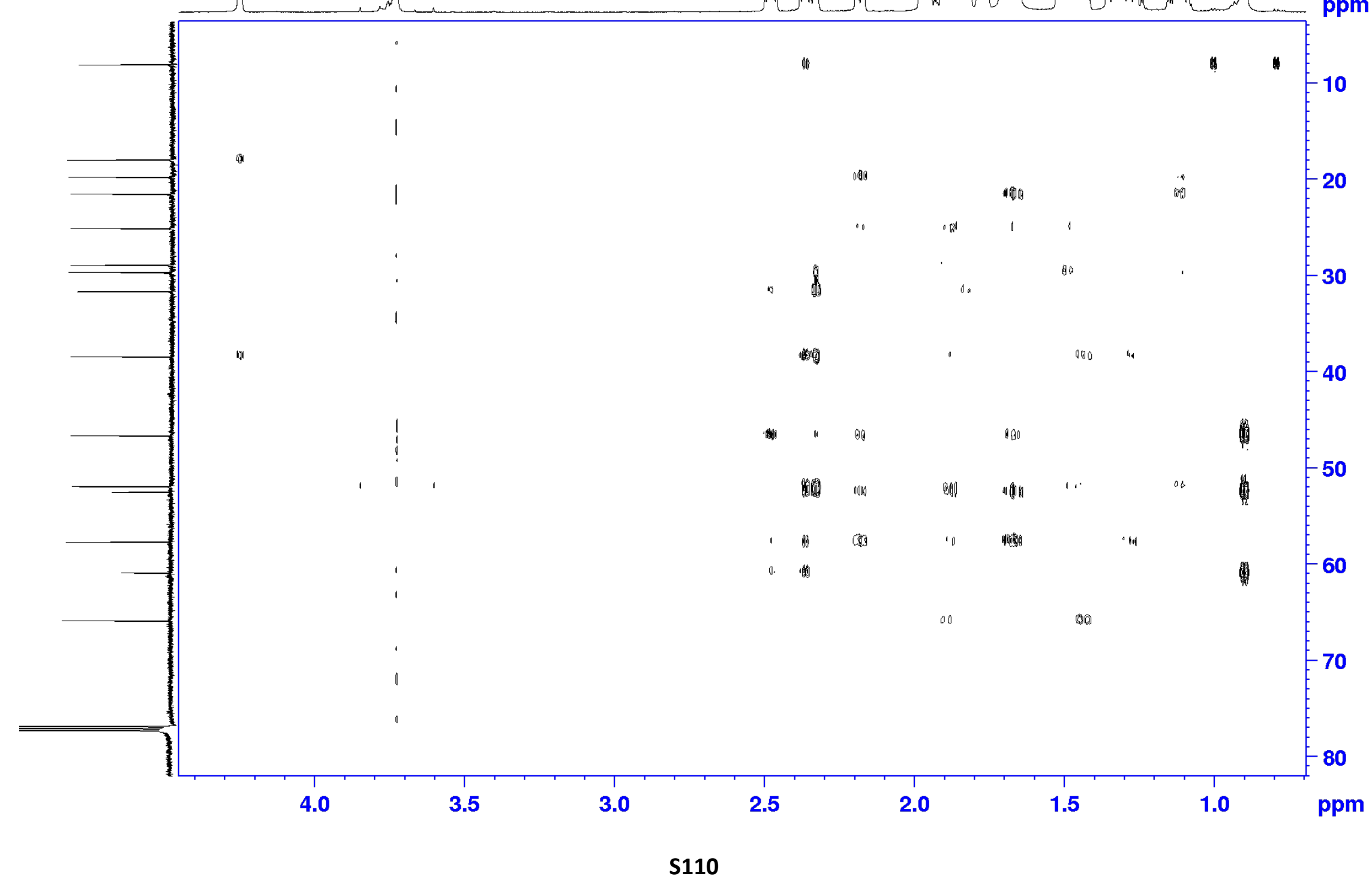




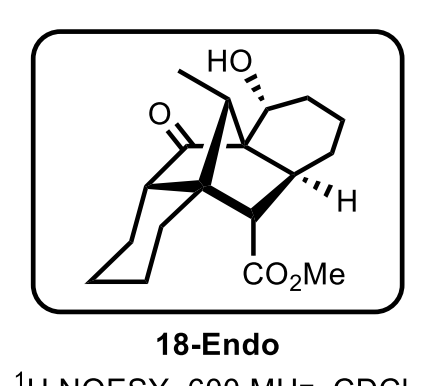

${ }^{1} \mathrm{H}$ NOESY, $600 \mathrm{MHz}, \mathrm{CDCl}_{3}$
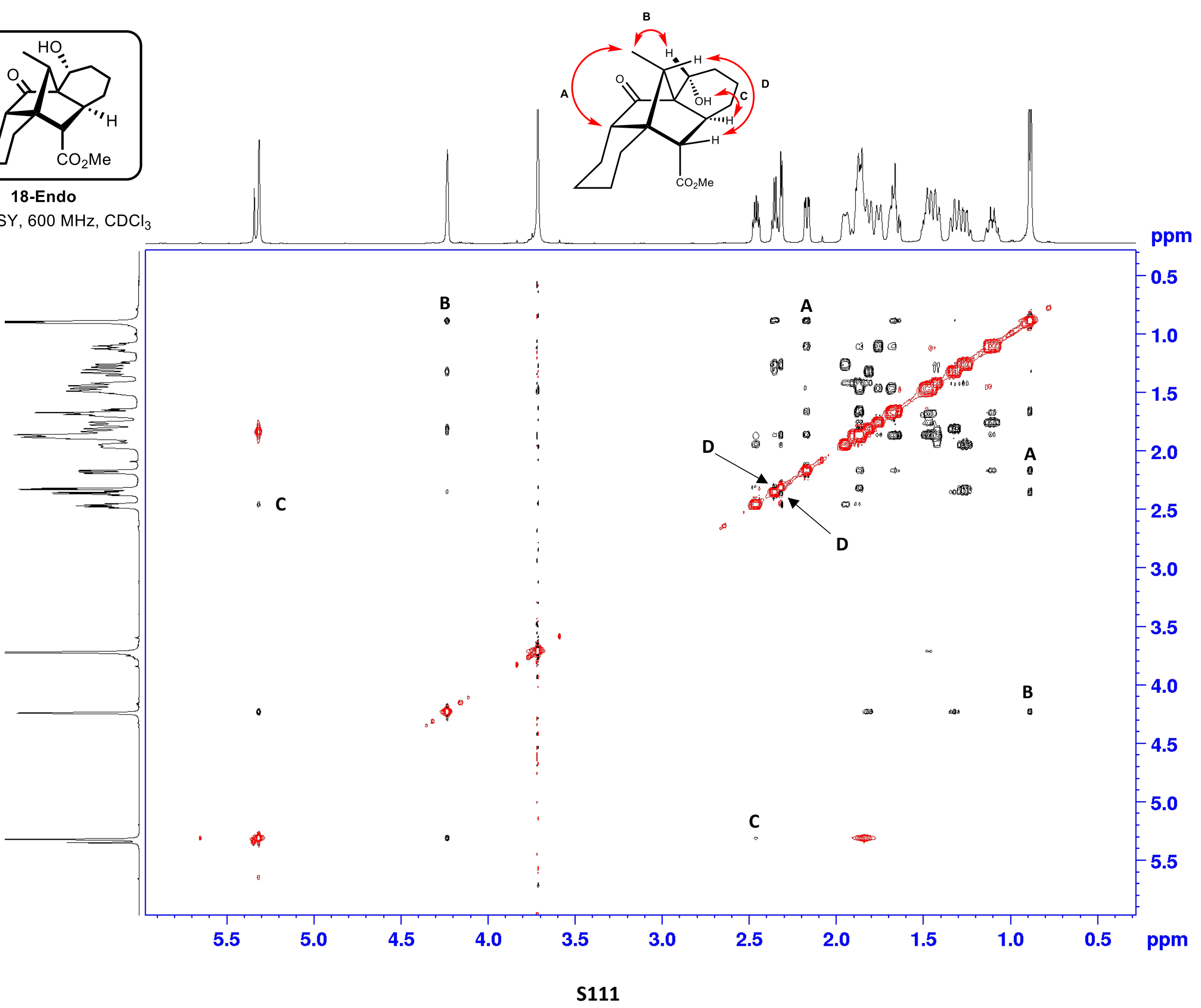


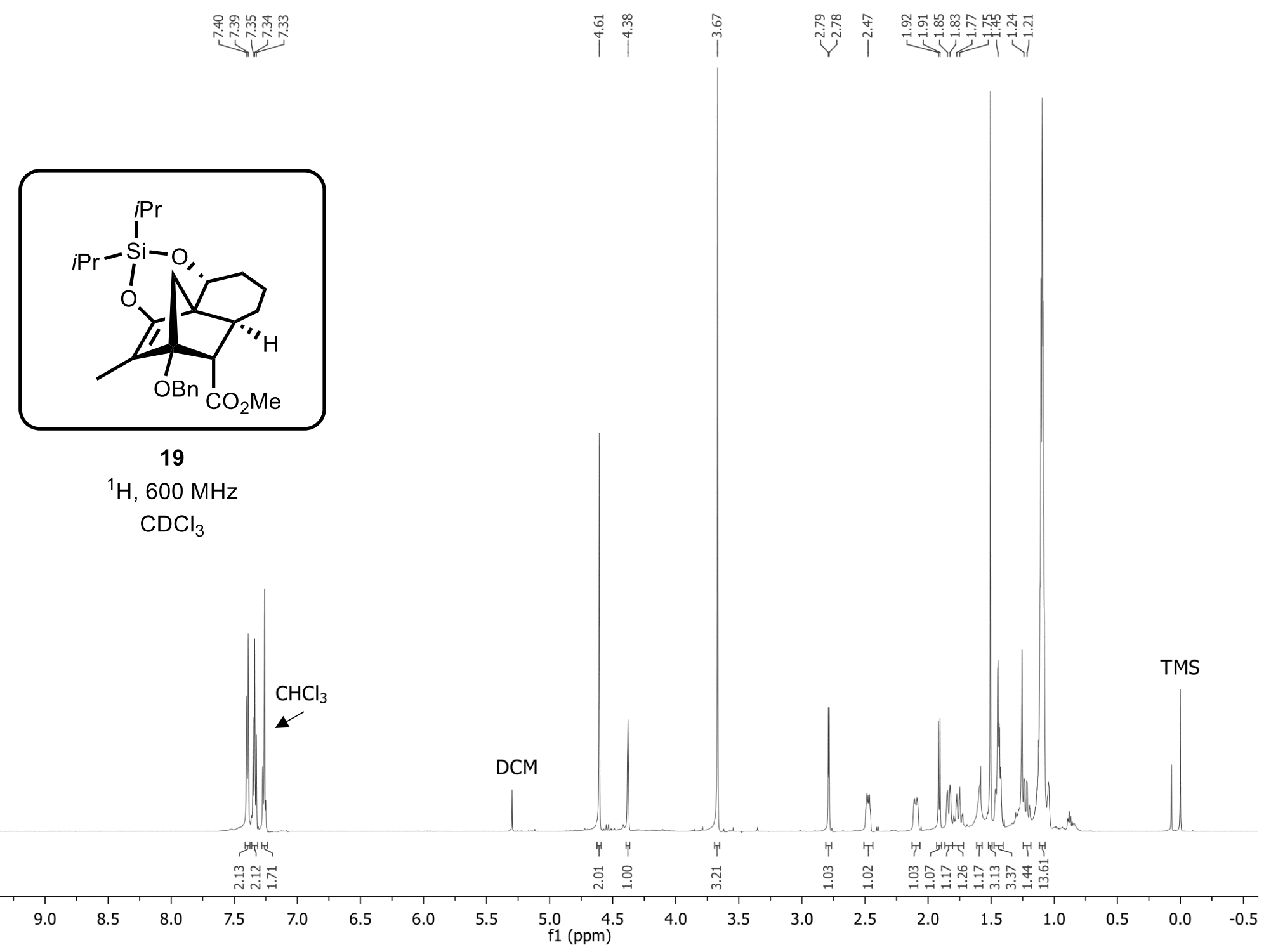

S112 


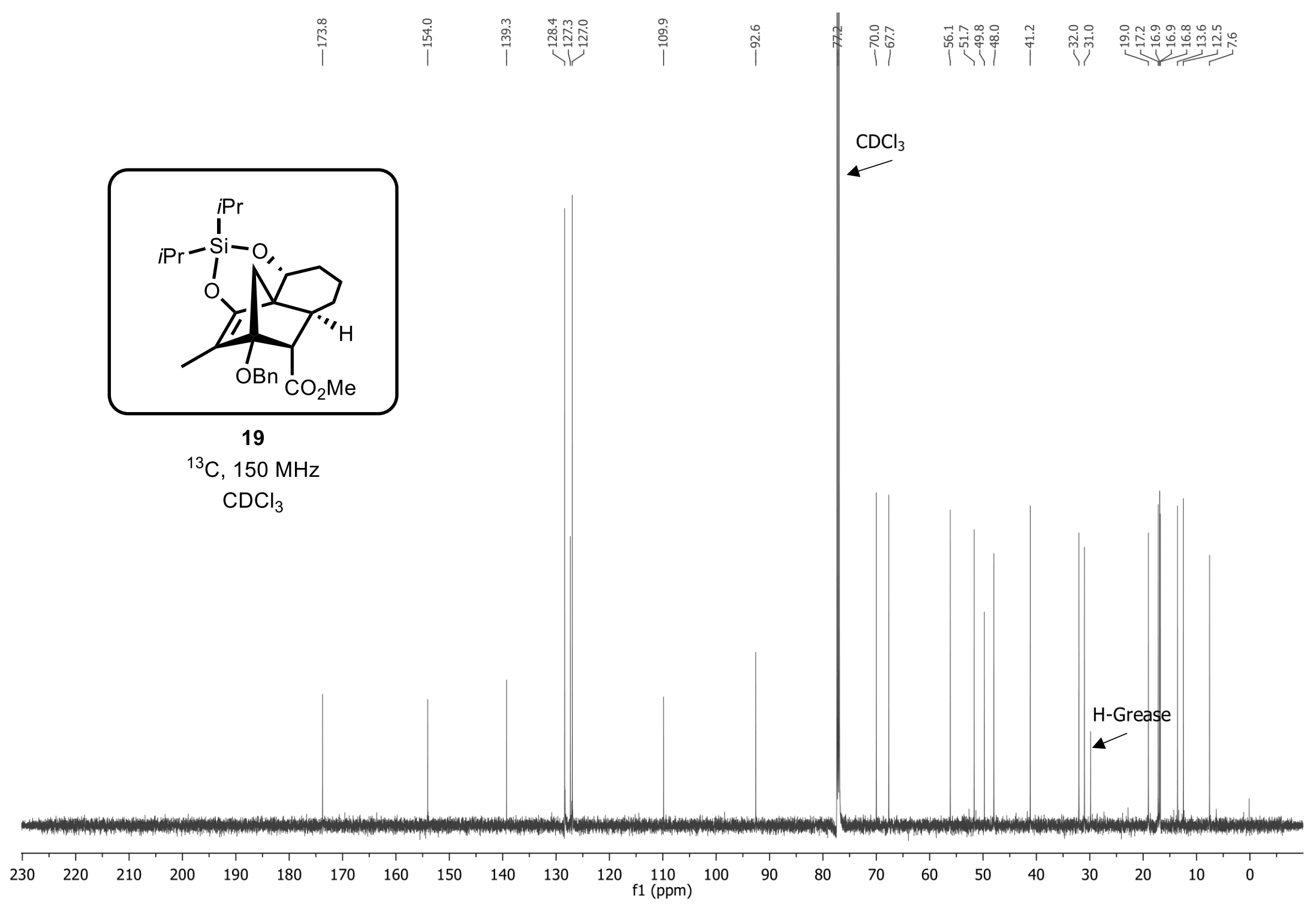




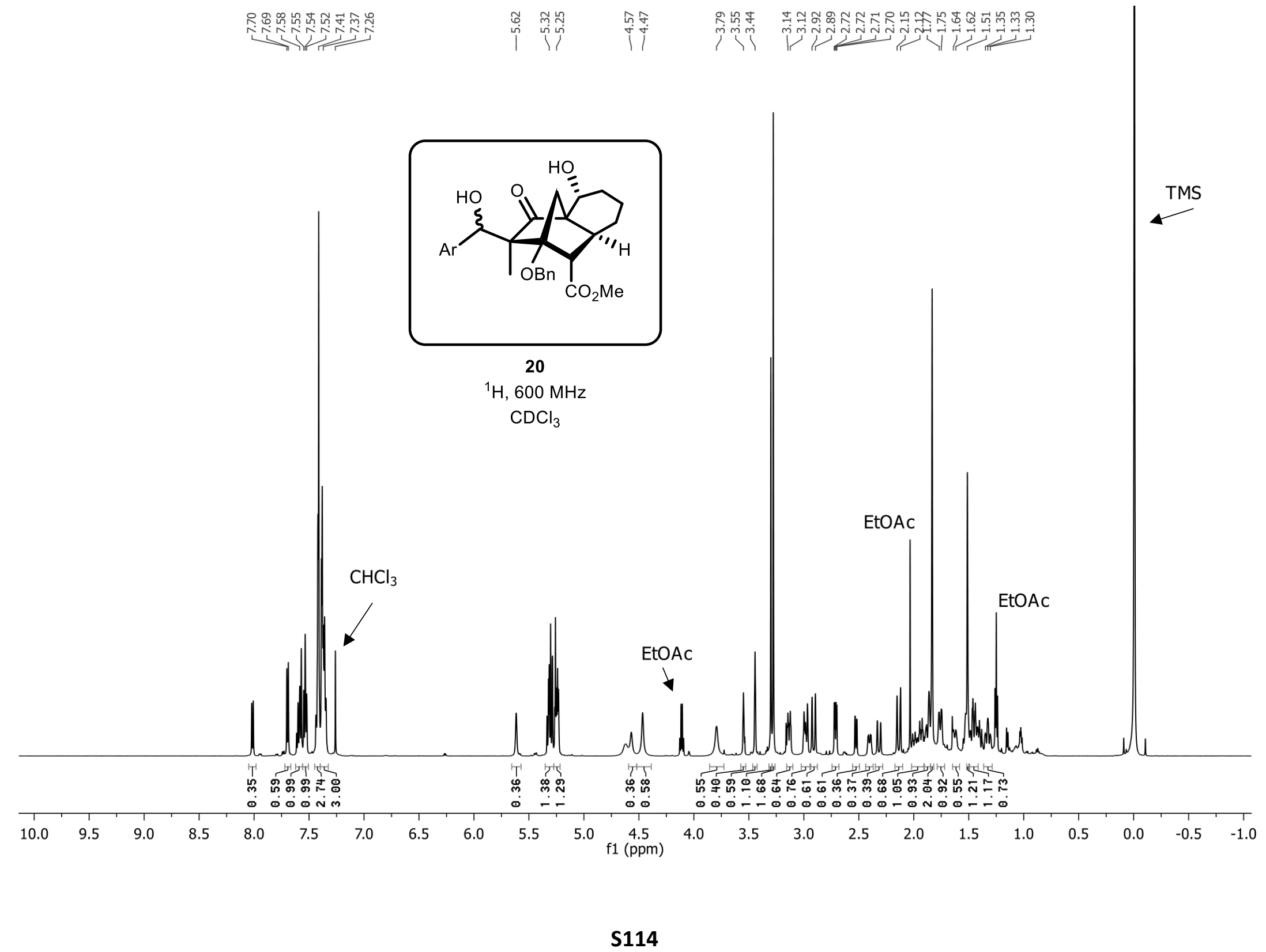




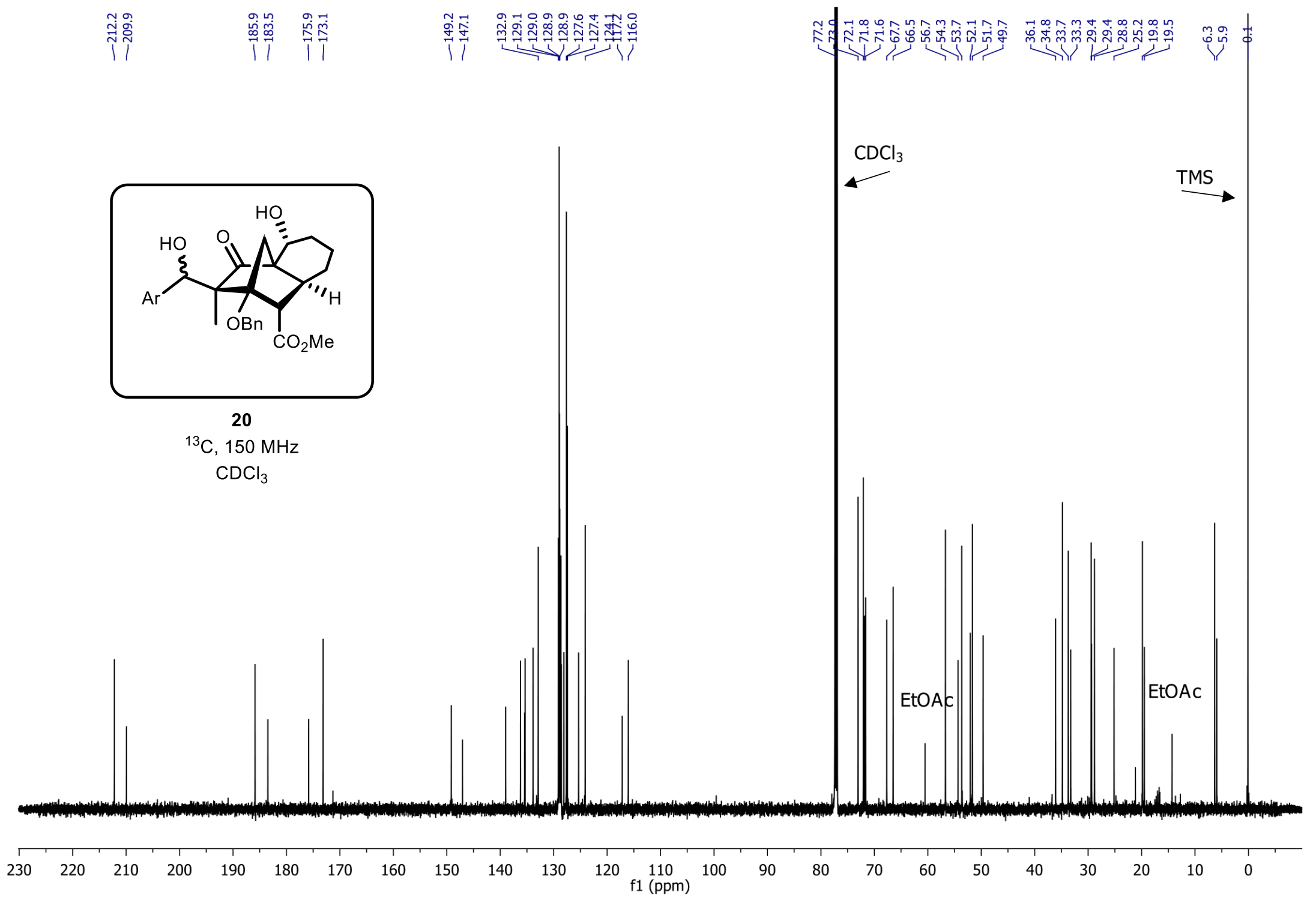




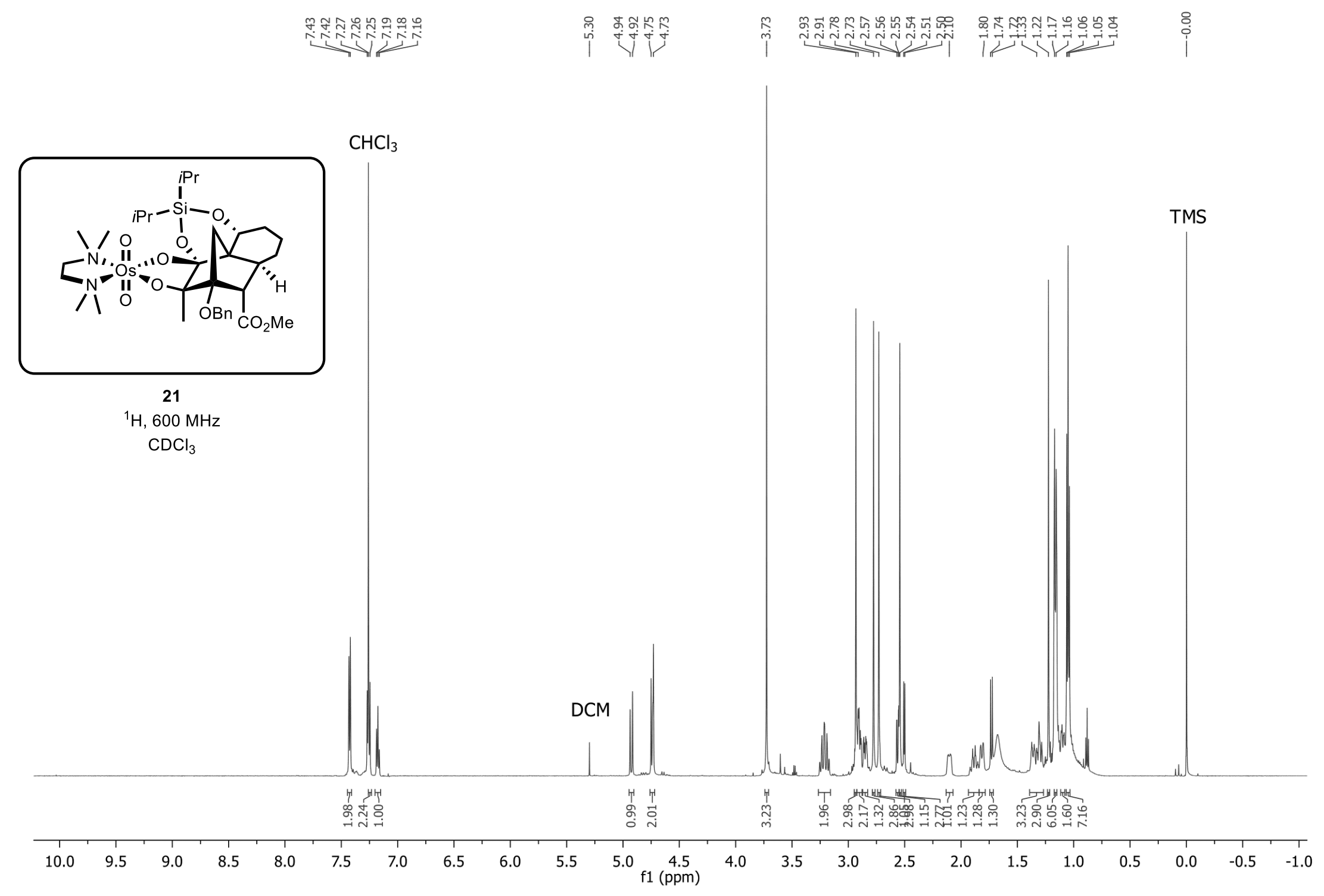

S116 


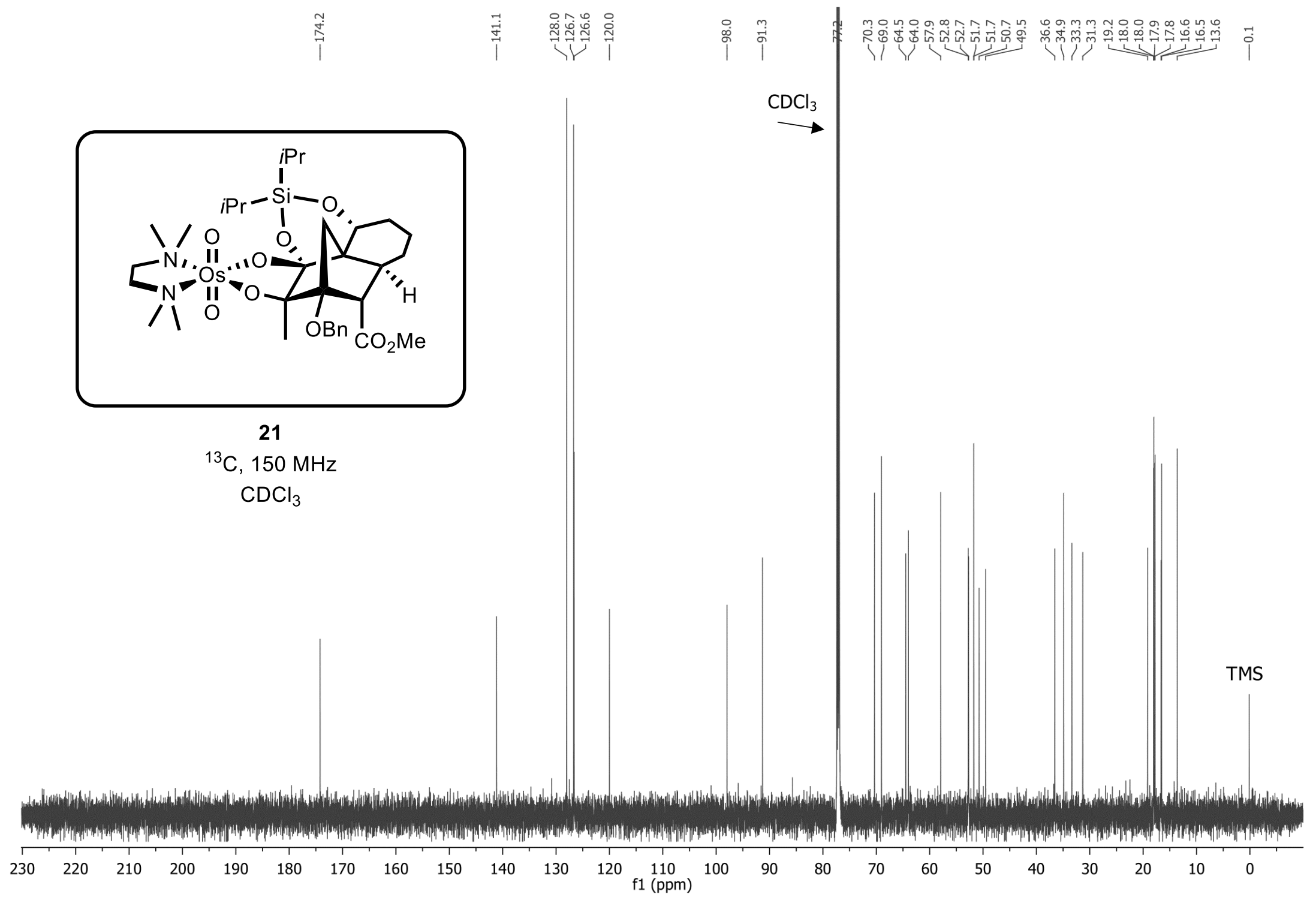




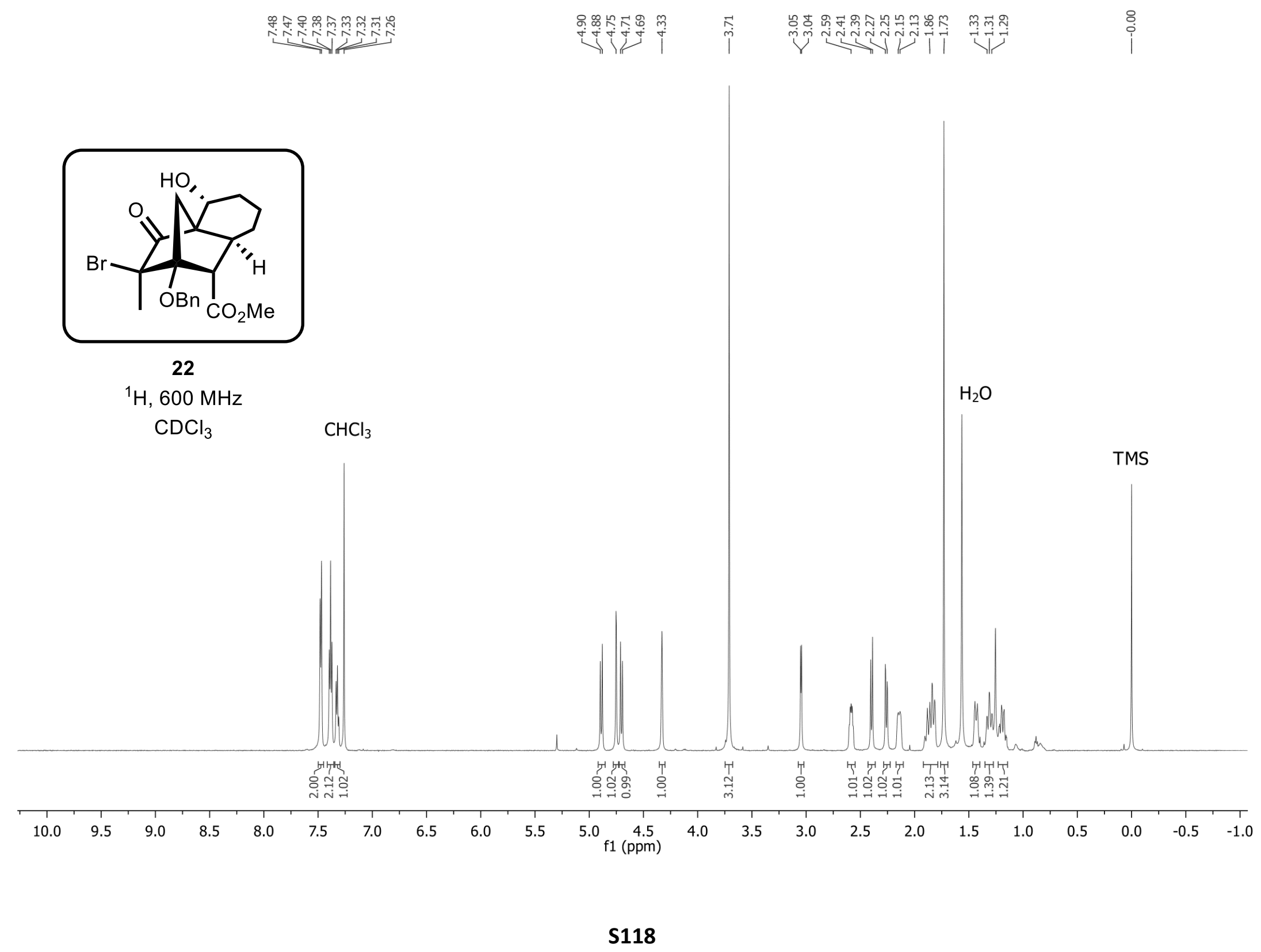




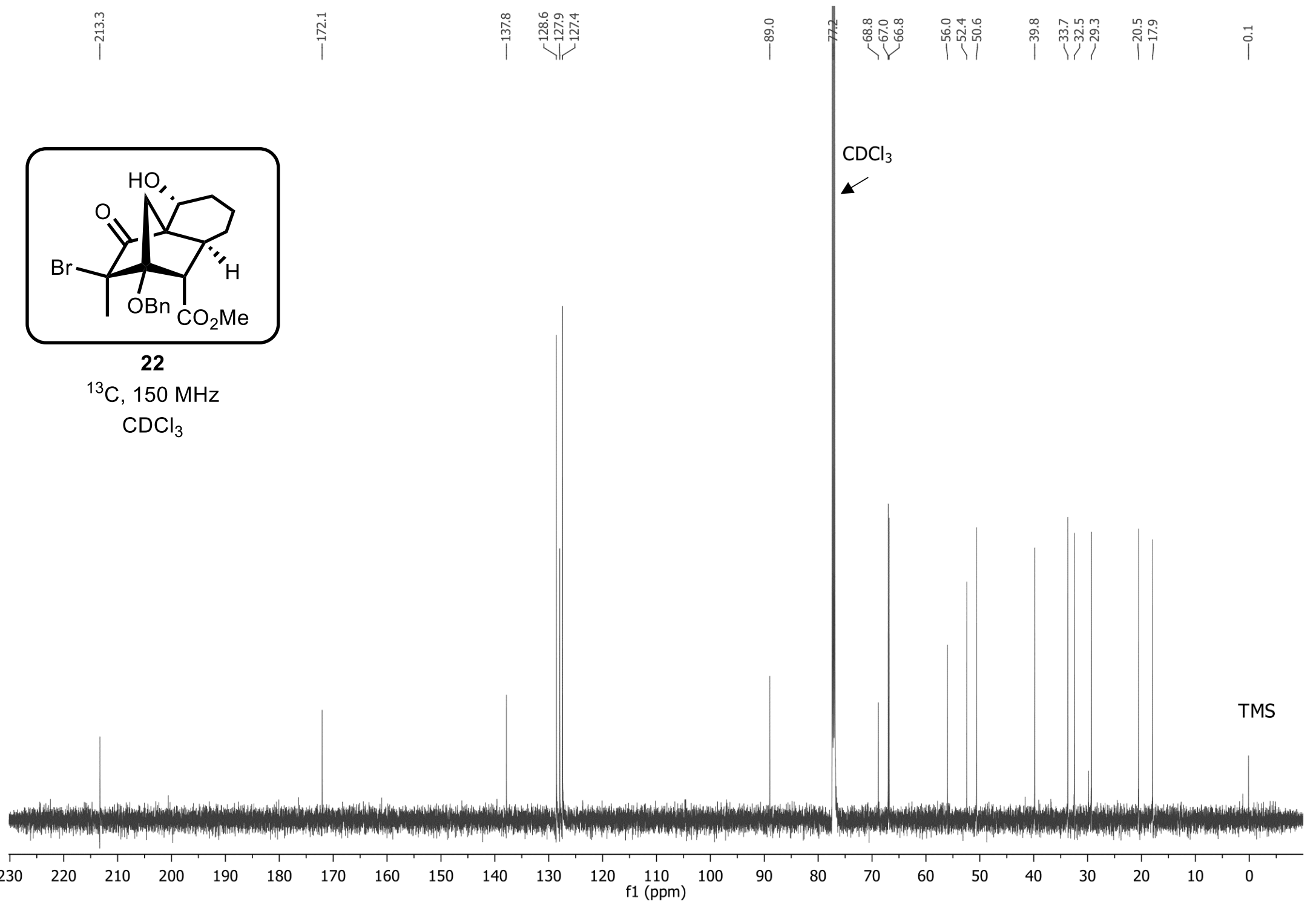




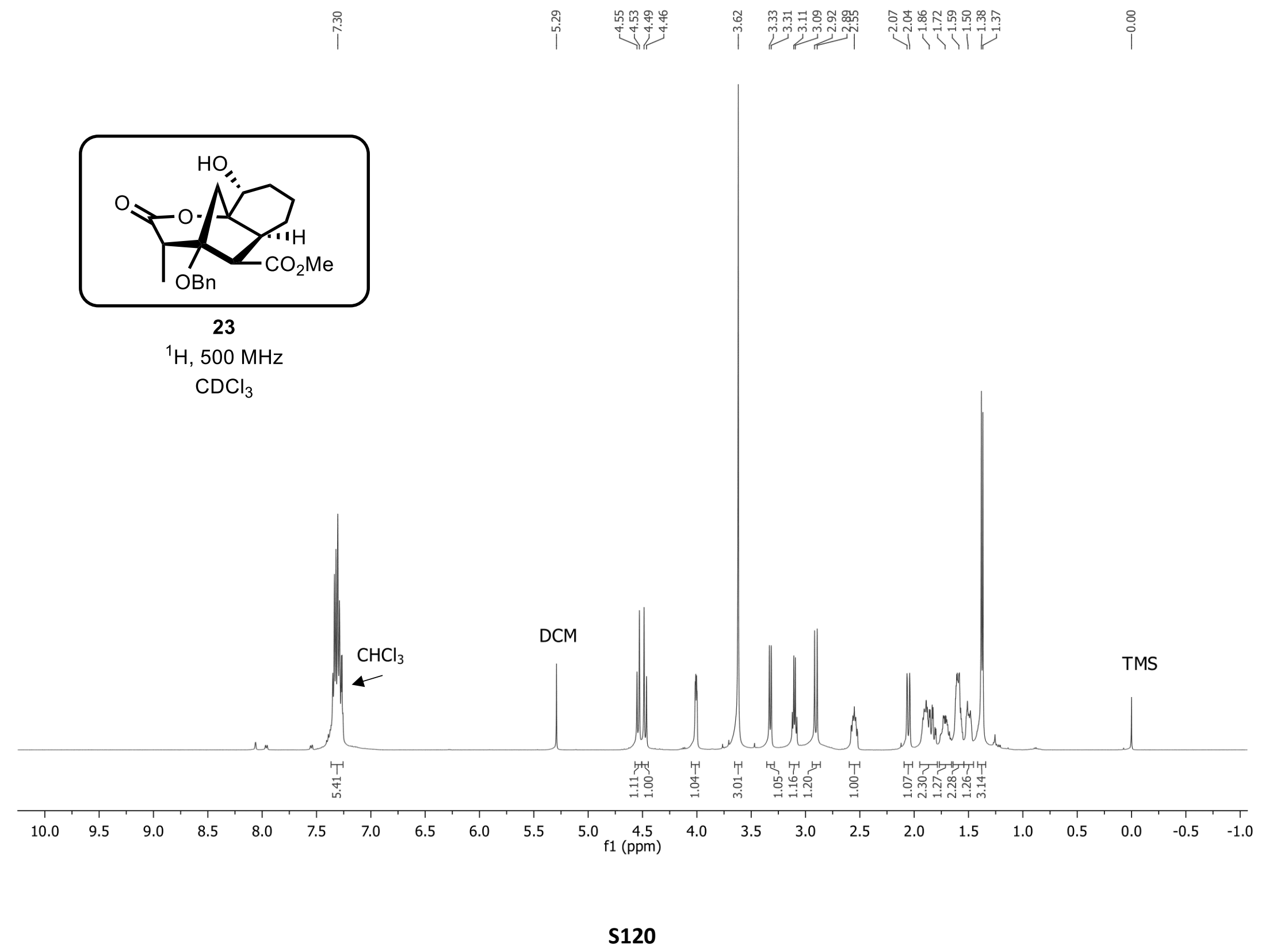




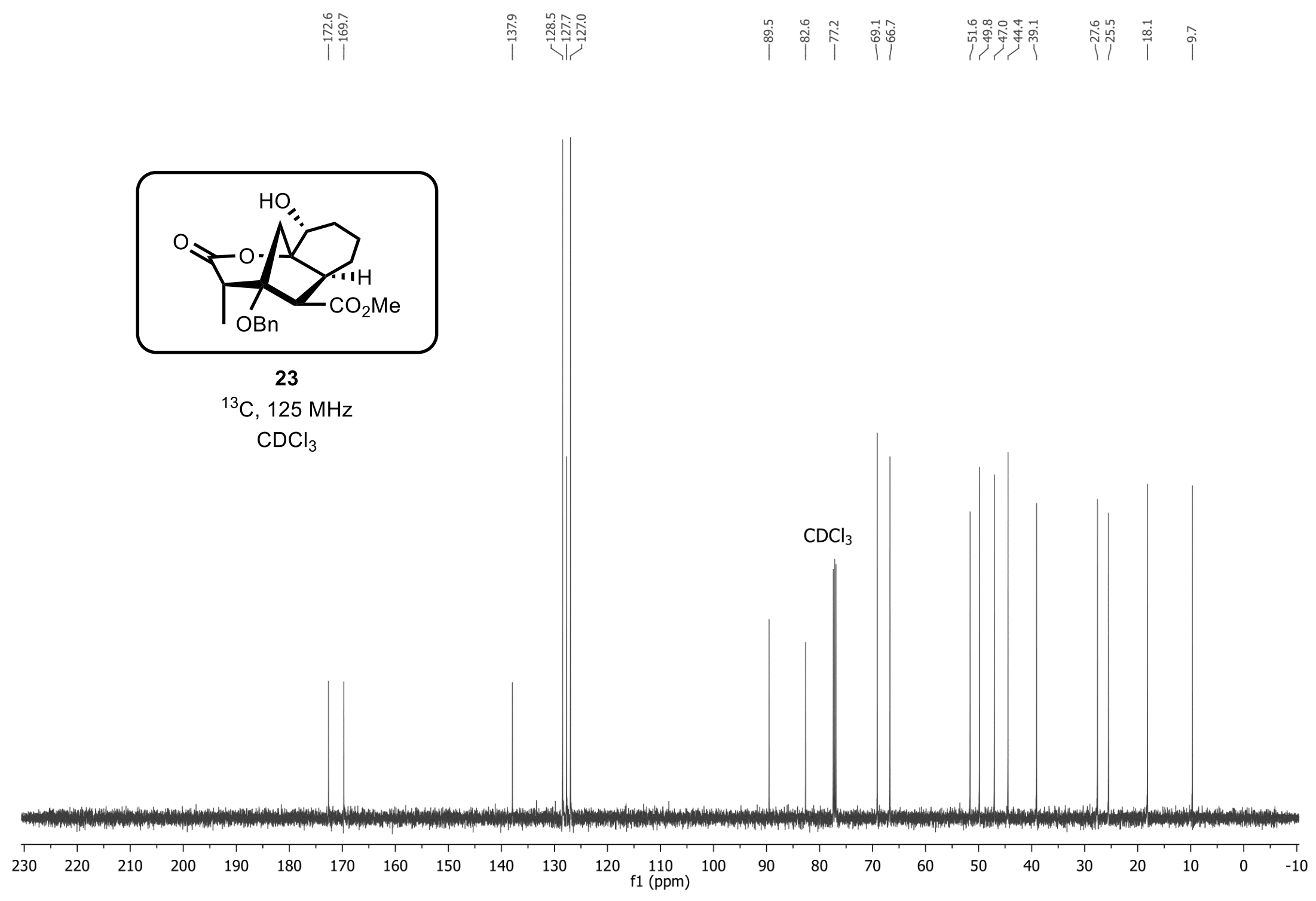

S121 
X-ray crystal data for 21

The thermal ellipsoid plot is shown at the 50\% probability level.

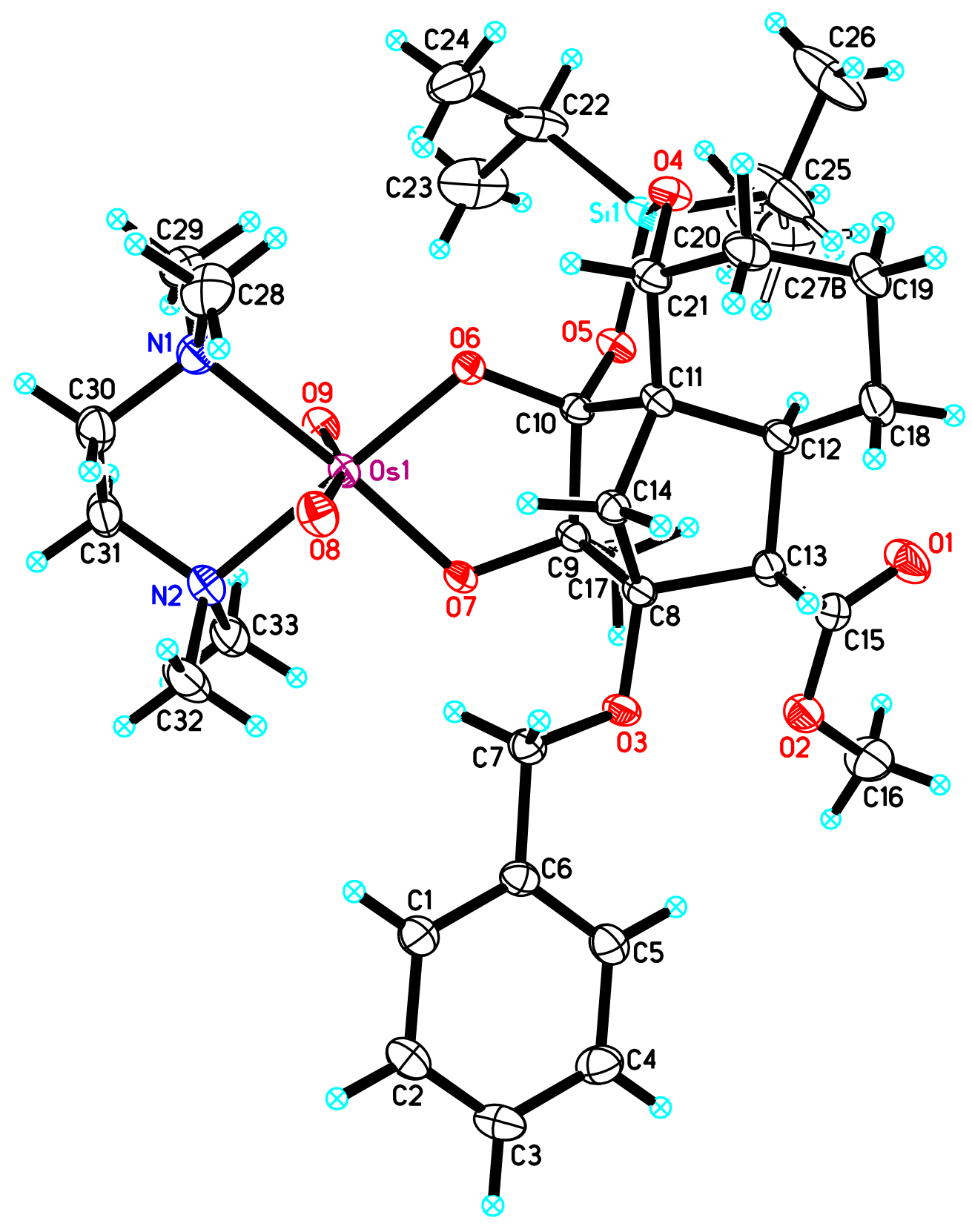




\section{X-ray Data Collection, Structure Solution and Refinement for sdr51.}

A brown crystal of approximate dimensions $0.188 \times 0.251 \times 0.277 \mathrm{~mm}$ was mounted in a cryoloop and transferred to a Bruker SMART APEX II diffractometer. The APEX2 program package was used to determine the unit-cell parameters and for data collection (20 sec/frame scan time). The raw frame data was processed using SAINT and SADABS to yield the reflection data file. Subsequent calculations were carried out using the SHELXTL program package. The diffraction symmetry was $2 / m$ and the systematic absences were consistent with the monoclinic space groups $C c$ and $C 2 / c$. It was later determined that space group $C 2 / c$ was correct

The structure was solved by direct methods and refined on $\mathrm{F}^{2}$ by full-matrix least-squares techniques. The analytical scattering factors ${ }^{5}$ for neutral atoms were used throughout the analysis. Hydrogen atoms were included using a riding model. Carbon atom C(27) was disordered and included with multiple components, partial site-occupancy-factors and isotropic thermal parameters.

Least-squares analysis yielded $\mathrm{wR} 2=0.0598$ and Goof $=1.077$ for 424 variables refined against 9110 data $(0.074 \AA), \mathrm{R} 1=0.0237$ for those 8147 data with $\mathrm{I}>2.0 \sigma(\mathrm{I})$. 
Table S1. Crystal data and structure refinement for 21.

Identification code

Empirical formula

Formula weight

Temperature

Wavelength

Crystal system

Space group

Unit cell dimensions

Volume

Z

Density (calculated)

Absorption coefficient

$F(000)$

Crystal color

Crystal size

Theta range for data collection

Index ranges

Reflections collected

Independent reflections

Completeness to theta $=25.242^{\circ}$

Absorption correction

Max. and min. transmission

Refinement method

Data / restraints / parameters

Goodness-of-fit on $F^{2}$

Final $\mathrm{R}$ indices $[\mid>2 \operatorname{sigma}(\mathrm{I})=8147$ data $]$
sdr51(Paul Carlson)

$\mathrm{C}_{33} \mathrm{H}_{54} \mathrm{~N}_{2} \mathrm{O}_{9} \mathrm{Os} \mathrm{Si}$

841.07

$133(2) \mathrm{K}$

$0.71073 \AA$

Monoclinic

$C 2 / c$

$a=36.7754(18) \AA$

$\alpha=90^{\circ}$.

$\mathrm{b}=12.2628(6) \AA$

$\beta=93.6849(8)^{\circ}$.

$c=15.6604(8) \AA$

$\gamma=90^{\circ}$.

$7047.8(6) \AA^{3}$

8

$1.585 \mathrm{Mg} / \mathrm{m}^{3}$

$3.707 \mathrm{~mm}^{-1}$

3424

brown

$0.277 \times 0.251 \times 0.188 \mathrm{~mm}^{3}$

1.751 to $28.697^{\circ}$

$-49 \leq h \leq 49,-16 \leq k \leq 16,-21 \leq I \leq 21$

49765

$9110[R($ int $)=0.0249]$

$100.0 \%$

Semi-empirical from equivalents

0.4335 and 0.3599

Full-matrix least-squares on $\mathrm{F}^{2}$

$9110 / 0$ / 424

1.077

$R 1=0.0237, w R 2=0.0578$ 
R indices (all data, $0.74 \AA$ )

Largest diff. peak and hole
$\mathrm{R} 1=0.0286, \mathrm{wR} 2=0.0598$

1.921 and -0.740 e. $\AA^{-3}$ 


\section{Calculations}

The minimum energy silacycle dienes, endo and exo transitions states, and endo and exo DielsAlder adducts for 1-8 are reported as the values in hartrees of the single point energies of the optimized structures calculated at $\omega \mathrm{B} 97 \mathrm{xd} / 6-311 \mathrm{G}(\mathrm{d}, \mathrm{p})$ with the thermal correction to Gibbs Free Energy $(\mathrm{SCF}(\omega \mathrm{B} 97 \mathrm{xd} / 6-311 \mathrm{G}(\mathrm{d}, \mathrm{p}))+($ Sum of electronic and thermal Free Energies $(\omega \mathrm{B} 97 \mathrm{xd} / 6-31 \mathrm{G}(\mathrm{d}))-\mathrm{SCF}(\omega \mathrm{B} 97 \mathrm{xd} / 6-31 \mathrm{G}(\mathrm{d})))$. The same energies are also shown in $\mathrm{kcal} / \mathrm{mol}$ relative to the silacycle diene. Geometries were optimized using $\omega \mathrm{B} 97 \mathrm{xd} / 6-31 \mathrm{G}(\mathrm{d})$ and included full frequency analysis. Single point energies were calculated using $\omega B 97 x d / 6-311+G(d, p)$ at the optimized geometry. All calculations were performed at $298.150 \mathrm{~K}$ and $1.00000 \mathrm{~atm}$.

Calculations were performed using Gaussian 16 and Spartan 18. 1,2 Images were processed with CYLView 2.0.3

\begin{tabular}{|c|c|c|c|c|c|c|}
\hline entry & Silacycle Diene & TS-endo & TS-exo & Endo preference & Endo-adduct & Exo-adduct \\
\hline 1 (hartree) & -1328.761073 & -1328.733902 & -1328.718441 & 0.01546101 & -1328.788613 & -1328.780656 \\
\hline 1 (rel. E: kcal/mol) & 0 & 17.05 & 26.75 & 9.70 (endo) & -17.28 & -12.29 \\
\hline 2 (hartree) & -1328.757084 & -1328.718128 & -1328.729245 & -0.01111659 & -1328.775757 & -1328.785883 \\
\hline 2 (rel. E: kcal/mol) & 0 & 24.44 & 17.47 & $-6.98(e x o)$ & -11.72 & -18.07 \\
\hline 3 (hartree) & -1289.475476 & -1289.448047 & -1289.437856 & 0.01019081 & -1289.492371 & -1289.482175 \\
\hline 3 (rel. E: kcal/mol) & 0 & 17.21 & 23.61 & 6.39 (endo) & -10.60 & -4.20 \\
\hline 4 (hartree) & -1880.453656 & -1880.422406 & -1880.406204 & 0.01620266 & -1880.482476 & -1880.475044 \\
\hline 4 (rel. E: kcal/mol) & 0 & 19.61 & 29.78 & 10.17 (endo) & -18.08 & -13.42 \\
\hline 5/6 (hartree) & -1174.976068 & -1174.942409 & -1174.928026 & 0.01438235 & -1175.008626 & -1175.000131 \\
\hline 5/6 (rel. E: kcal/mol) & 0 & 21.12 & 30.15 & 9.02 (endo) & -20.43 & -15.10 \\
\hline $7 / 8$ (hartree) & -1370.234116 & -1370.201387 & -1370.191312 & 0.0100747 & -1370.256715 & -1370.252169 \\
\hline 7/8 (rel. E: kcal/mol) & 0 & 20.54 & 26.86 & 6.32 (endo) & -14.18 & -11.33 \\
\hline
\end{tabular}

Values in hartrees are the single point energies of the optimized structures calculated at $\omega \mathrm{B} 97 \times \mathrm{d} / 6-311 \mathrm{G}(\mathrm{d}, \mathrm{p})$ with the thermal correction to Gibbs Free Energy $(\operatorname{SCF}(\omega B 97 x d / 6-311 G(d, p))+($ Sum of electronic and thermal Free Energies $(\omega B 97 x d / 6-31 G(d))-S C F$ $(\omega \mathrm{B} 97 \mathrm{xd} / 6-31 \mathrm{G}(\mathrm{d})))$. The same energies are also shown in $\mathrm{kcal} / \mathrm{mol}$ relative to the silacycle diene. The endo preference corresponds to TS-exo - TS-endo. 
Figure S3. Reaction Profile for the Silacycle IMDA reaction for Entry 1 - Figure 1.

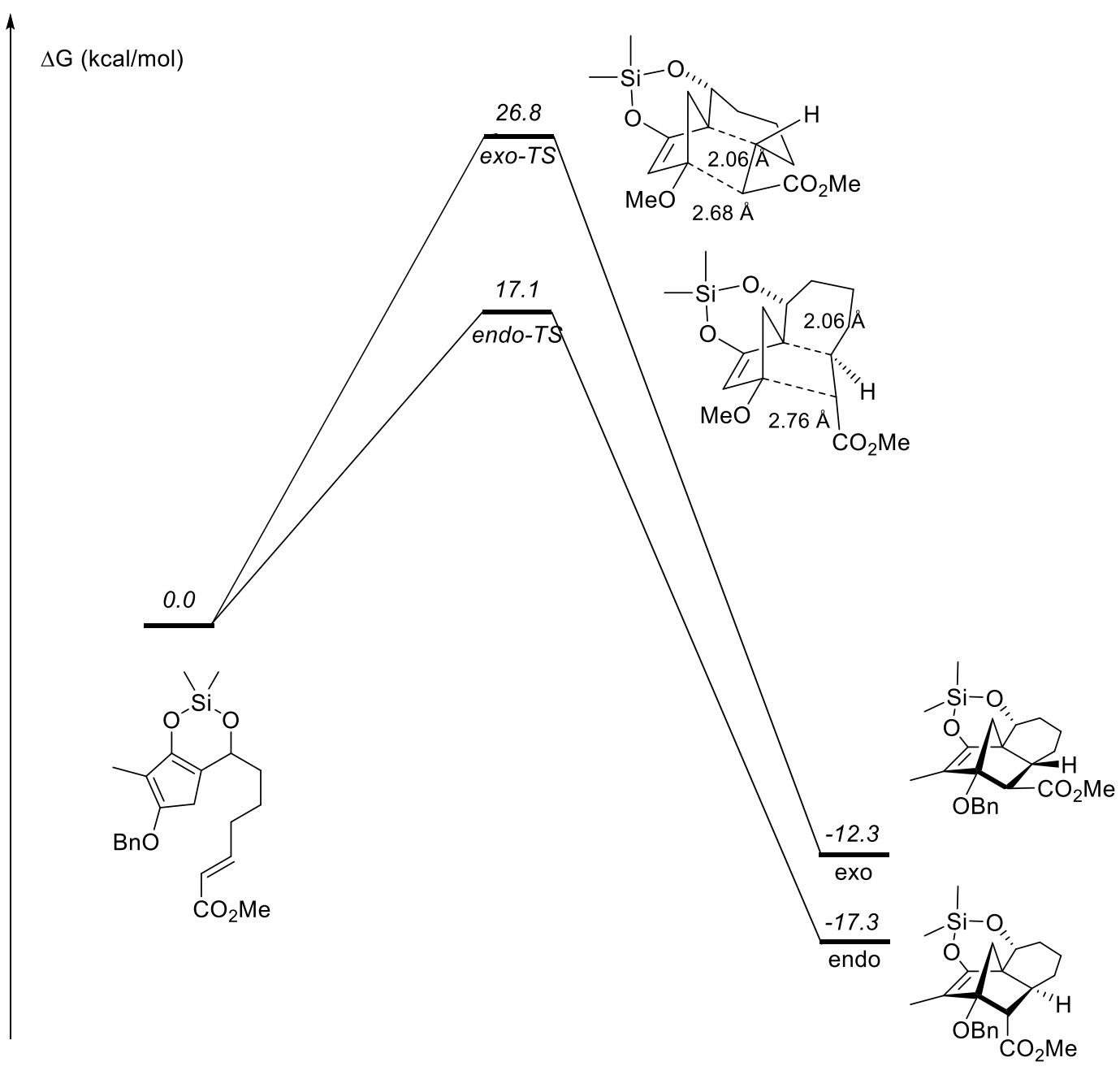




\section{Calculated silacycle diene for entry 1:}

Single point: SCF $(\omega \mathrm{B} 97 \mathrm{xd} / 6-311 \mathrm{G}(\mathrm{d}, \mathrm{p}))=-1329.120142$

Sum of electronic and thermal Free Energies $(\omega \mathrm{B} 97 \mathrm{xd} / 6-31 \mathrm{G}(\mathrm{d}))=-1367.707501$ $\operatorname{SCF}(\omega B 97 x d / 6-31 G(d))=-1328.822961$

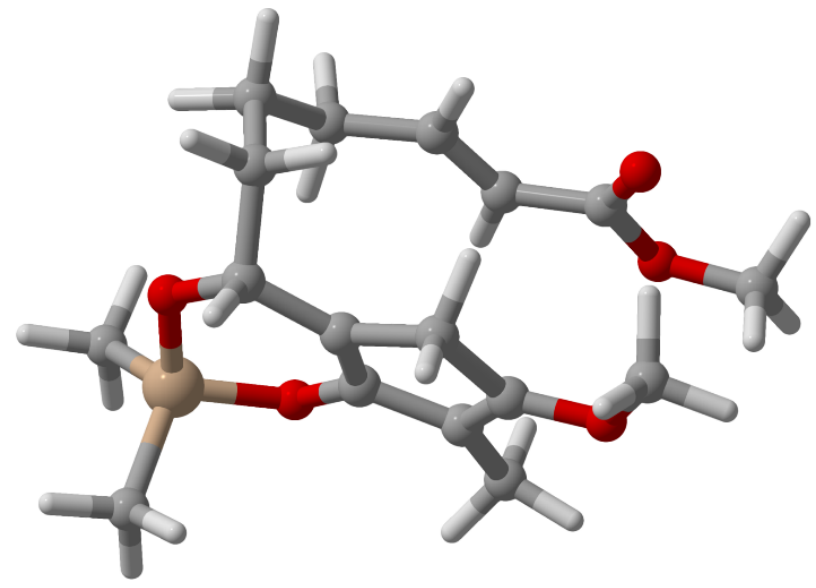

49

E_17_sm_M0005

$\begin{array}{llll}\mathrm{O}^{-} & -1.2701110 & -2.9776450 & 0.7015920\end{array}$

C $\quad-1.0160120 \quad-1.7771460 \quad 0.1349630$

$\begin{array}{llll}\text { C } & -1.6817780 & -0.4827860 & 0.5308320\end{array}$

$\begin{array}{llll}\mathrm{C} & -1.0396050 & 0.5163460 & -0.3900590\end{array}$

$\begin{array}{llll}\text { C } & -0.1602050 & -0.1531710 & -1.1648130\end{array}$

$\begin{array}{llll}\text { C } & -0.1166940 & -1.5829110 & -0.8511410\end{array}$

$\begin{array}{llll}\text { C } & -1.3455200 & 1.9878180 & -0.4215090\end{array}$

O $\quad-0.5870520 \quad 2.6553430 \quad-1.4310950$

$\begin{array}{llll}\mathrm{Si} & 0.0867970 & 1.8627980 & -2.7215210\end{array}$

$\begin{array}{llll}\mathrm{O} & 0.6401300 & 0.3789050 & -2.1299240\end{array}$

$\begin{array}{llll}\text { C } & -2.1855060 & -3.0031500 & 1.7825740\end{array}$

$\begin{array}{llll}\text { C } & 0.7966070 & -2.5802370 & -1.4833780\end{array}$

$\begin{array}{llll}\mathrm{C} & -1.1073370 & 2.7061890 & 0.9191560\end{array}$

$\mathrm{H} \quad-2.4124920 \quad 2.1194830 \quad-0.6660400$

C $\quad 0.3355880 \quad 3.1317590 \quad 1.2182380$

C $\quad 1.4287240 \quad 2.0569670 \quad 1.1422920$

C $\quad 1.1557860 \quad 0.8225650 \quad 1.9490170$

$\begin{array}{llll}\text { C } & 1.6021760 & -0.3900840 & 1.6181500\end{array}$

$\begin{array}{llll}\mathrm{C} & 1.5593160 & 2.8331100 & -3.3049470\end{array}$

$\begin{array}{llll}\text { C } & -1.1776930 & 1.5196470 & -4.0498720\end{array}$

C $\quad 1.2192660 \quad-1.5889400 \quad 2.3842100$

$\begin{array}{llll}\mathrm{O} & 0.4101980 & -1.6249140 & 3.2916040\end{array}$

$\begin{array}{llll}\mathrm{O} & 1.8667900 & -2.6734100 & 1.9285840\end{array}$

$\begin{array}{llll}\text { C } & 1.4791880 & -3.9156780 & 2.5077090\end{array}$

$\mathrm{H} \quad-2.7733610 \quad-0.5277850 \quad 0.4121890$

$\mathrm{H} \quad-1.4774830 \quad-0.2673890 \quad 1.5892310$

$\mathrm{H} \quad-3.1715110 \quad-2.6287060 \quad 1.4806540$ 


$\begin{array}{rrrr}\mathrm{H} & -1.8047280 & -2.4255950 & 2.6308660 \\ \mathrm{H} & -2.2782910 & -4.0513900 & 2.0712440 \\ \mathrm{H} & 0.4319870 & -3.5966380 & -1.3123810 \\ \mathrm{H} & 0.8783140 & -2.4114250 & -2.5618220 \\ \mathrm{H} & 1.8067130 & -2.5142010 & -1.0604500 \\ \mathrm{H} & -1.7214110 & 3.6146220 & 0.9344100 \\ \mathrm{H} & -1.4934860 & 2.0631710 & 1.7200190 \\ \mathrm{H} & 0.3436260 & 3.5725770 & 2.2239080 \\ \mathrm{H} & 0.6092490 & 3.9334040 & 0.5252340 \\ \mathrm{H} & 1.6052550 & 1.7661340 & 0.1012950 \\ \mathrm{H} & 2.3638210 & 2.5127010 & 1.4991320 \\ \mathrm{H} & 0.5522970 & 0.9188730 & 2.8523180 \\ \mathrm{H} & 2.2049320 & -0.5502240 & 0.7290690 \\ \mathrm{H} & 2.2625830 & 2.9894800 & -2.4809860 \\ \mathrm{H} & 1.2582780 & 3.8144280 & -3.6863320 \\ \mathrm{H} & 2.0847430 & 2.3041420 & -4.1071000 \\ \mathrm{H} & -1.9779340 & 0.8861590 & -3.6519080 \\ \mathrm{H} & -0.7282210 & 0.9980880 & -4.9016900 \\ \mathrm{H} & -1.6309480 & 2.4468850 & -4.4165250 \\ \mathrm{H} & 0.4643730 & -4.1680020 & 2.1913450 \\ \mathrm{H} & 2.1862430 & -4.6519100 & 2.1255290 \\ \mathrm{H} & 1.5244020 & -3.8682570 & 3.5981280\end{array}$

\section{Calculated endo product for entry 1:}

Single point: SCF $(\omega B 97 x d / 6-311 G(d, p))=-1329.155501$

Sum of electronic and thermal Free Energies $(\omega \mathrm{B} 97 \mathrm{xd} / 6-31 \mathrm{G}(\mathrm{d}))=-1328.495372$ $\operatorname{SCF}(\omega \mathrm{B} 97 \mathrm{xd} / 6-31 \mathrm{G}(\mathrm{d}))=-1328.862261$

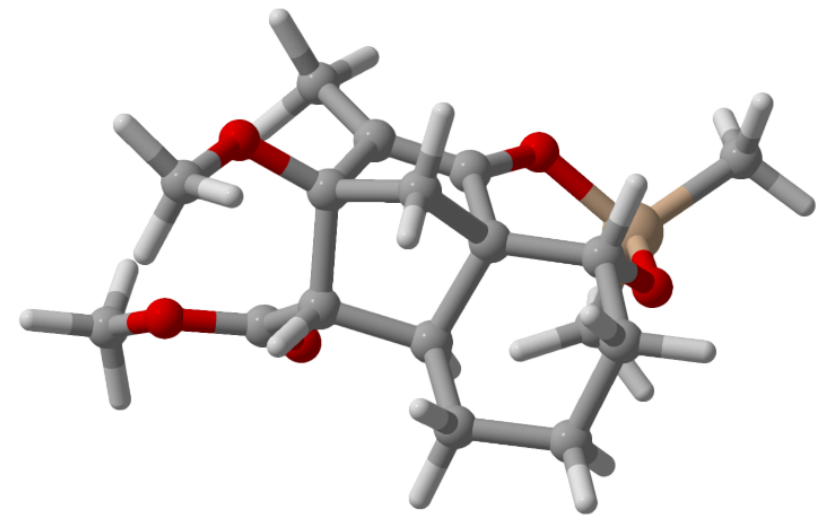

49

E_17_endo_M0003

$\begin{array}{llrr}\mathrm{O}^{-} & -1.2399760 & 0.8285580 & -1.9908380 \\ \mathrm{C} & -1.1448850 & 0.2717260 & -0.7596900 \\ \mathrm{C} & -1.5417260 & -0.9457670 & -0.3830150 \\ \mathrm{C} & -1.1051390 & -1.0740750 & 1.0660180\end{array}$




\begin{tabular}{|c|c|c|c|}
\hline $\mathrm{C}$ & -1.2744640 & 0.3811460 & 1.5644380 \\
\hline $\mathrm{C}$ & -0.4488970 & 0.9671610 & 0.4057880 \\
\hline $\mathrm{C}$ & -2.1192680 & -2.0567150 & -1.1903060 \\
\hline $\mathrm{C}$ & 0.4836070 & -1.1811490 & 1.0764470 \\
\hline $\mathrm{C}$ & 0.9220620 & 0.2298920 & 0.6261950 \\
\hline $\mathrm{C}$ & -0.3165490 & 2.4788380 & 0.2420450 \\
\hline $\mathrm{C}$ & 0.5601010 & 3.1027840 & 1.3227420 \\
\hline $\mathrm{O}$ & 0.2936480 & 2.7704090 & -1.0174530 \\
\hline $\mathrm{H}$ & -1.3177600 & 2.9336870 & 0.2671680 \\
\hline $\mathrm{H}$ & 1.4669180 & 0.1692040 & -0.3194890 \\
\hline $\mathrm{C}$ & 0.9788830 & -2.2956340 & 0.1945380 \\
\hline $\mathrm{O}$ & 1.4100830 & -2.1795430 & -0.9303580 \\
\hline $\mathrm{O}$ & -1.8090180 & -2.0926440 & 1.7015820 \\
\hline $\mathrm{C}$ & -1.4574240 & -2.3637740 & 3.0364690 \\
\hline $\mathrm{Si}$ & -0.1505390 & 2.0315290 & -2.4366480 \\
\hline $\mathrm{C}$ & 1.3545330 & 1.2643990 & -3.2222830 \\
\hline $\mathrm{C}$ & -1.0626860 & 3.1954750 & -3.5665800 \\
\hline $\mathrm{C}$ & 1.9365650 & 2.436 & 1.3728770 \\
\hline $\mathrm{C}$ & 1.8073920 & 0.939 & 1.6588740 \\
\hline $\mathrm{O}$ & 0.8503410 & -3.484 & 0.8103300 \\
\hline $\mathrm{C}$ & 1.2085270 & -4.6203160 & 0.0275430 \\
\hline $\mathrm{H}$ & -0.8439310 & 0.572 & 2.5538850 \\
\hline $\mathrm{H}$ & -2.3236790 & 0.6868210 & 1.5488850 \\
\hline $\mathrm{H}$ & -1.4157610 & -2.8951510 & -1.2691710 \\
\hline $\mathrm{H}$ & -2.3456770 & -1.7104260 & -2.2020380 \\
\hline $\mathrm{H}$ & -3.0344580 & -2.4448890 & -0.7316000 \\
\hline $\mathrm{H}$ & 0.8211050 & -1.3846440 & 2.0979900 \\
\hline $\mathrm{H}$ & 0.6544380 & 4.1741660 & 1.1174330 \\
\hline $\mathrm{H}$ & 0.0542160 & 2.9978540 & 2.2920650 \\
\hline $\mathrm{H}$ & -1.4348760 & -1.4532110 & 3.6515780 \\
\hline $\mathrm{H}$ & -0.4863990 & -2.8703360 & 3.1046960 \\
\hline $\mathrm{H}$ & -2.2285340 & -3.0299010 & 3.4291560 \\
\hline $\mathrm{H}$ & 2.1990240 & 1.9620140 & -3.2307640 \\
\hline $\mathrm{H}$ & 1.6541720 & 0.3664720 & -2.6701950 \\
\hline $\mathrm{H}$ & 1.1500210 & 0.9651350 & -4.2560880 \\
\hline $\mathrm{H}$ & -0.4092470 & 4.0082250 & -3.9017170 \\
\hline $\mathrm{H}$ & -1.9227380 & 3.6388070 & -3.0548710 \\
\hline $\mathrm{H}$ & -1.4298580 & 2.6692410 & -4.4544370 \\
\hline $\mathrm{H}$ & 2.4402630 & 2.5904100 & 0.4118020 \\
\hline $\mathrm{H}$ & 2.5551930 & 2.9124640 & 2.1416530 \\
\hline $\mathrm{H}$ & 2.7951730 & 0.4630080 & 1.6738820 \\
\hline $\mathrm{H}$ & 1.3866800 & 0.8070980 & 2.6658940 \\
\hline $\mathrm{H}$ & 2.2548840 & -4.5616750 & -0.2823160 \\
\hline $\mathrm{H}$ & 1.0471800 & -5.4845780 & 0.6712660 \\
\hline $\mathrm{H}$ & 0.5784830 & -4.6858910 & -0.8633840 \\
\hline
\end{tabular}




\section{Calculated exo product for entry 1:}

Single point: SCF $(\omega \mathrm{B} 97 \mathrm{xd} / 6-311 \mathrm{G}(\mathrm{d}, \mathrm{p}))=-1329.148714$

Sum of electronic and thermal Free Energies $(\omega \mathrm{B} 97 \mathrm{xd} / 6-31 \mathrm{G}(\mathrm{d}))=-1328.487723$

$\operatorname{SCF}(\omega \mathrm{B} 97 \mathrm{xd} / 6-31 \mathrm{G}(\mathrm{d}))=-1328.855781$

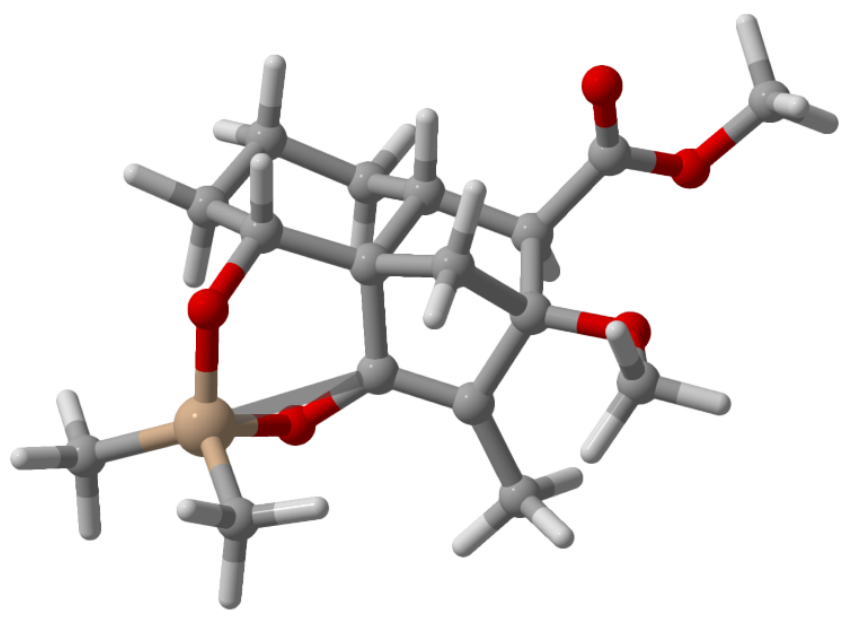

49

E_17_exo_M0001

$\mathrm{O}^{-}-0.40 \overline{0} 8930 \quad-0.4049510 \quad-2.3080440$

C $\quad 0.0154080 \quad-0.4551580 \quad-1.0028980$

C $\quad-0.4032250 \quad-1.5059060 \quad-0.2933290$

C $\quad-0.7382090 \quad-0.9319690 \quad 1.0827480$

$\begin{array}{llll}\mathrm{C} & -1.2695320 & 0.4759970 & 0.7018190\end{array}$

$\begin{array}{llll}\text { C } & -0.0128330 & 0.7982450 & -0.1297410\end{array}$

$\begin{array}{llll}\text { C } & 0.1374060 & 2.0834480 & -0.9406910\end{array}$

$\begin{array}{llll}\mathrm{C} & 1.0509710 & 0.6893520 & 1.0112280\end{array}$

C $\quad 0.6373240 \quad-0.5934520 \quad 1.7631280$

$\begin{array}{llll}\text { C } & -0.4023000 & -2.9578140 & -0.6300810\end{array}$

$\begin{array}{llll}\mathrm{O} & -1.4973280 & -1.7547360 & 1.9199140\end{array}$

C $\quad-2.8632890 \quad-1.8348100 \quad 1.5860430$

O $\quad-0.6708130 \quad 2.0229180 \quad-2.1178100$

$\mathrm{H} \quad-0.2343810 \quad 2.9291730 \quad-0.3503060$

$\begin{array}{llll}\text { C } & 2.4772020 & 0.8283400 & 0.5087370\end{array}$

$\begin{array}{llll}\mathrm{H} & 0.8580290 & 1.5332800 & 1.6863040\end{array}$

$\begin{array}{llll}\text { C } & 0.4454640 & -0.3735980 & 3.2461390\end{array}$

O $\quad-0.0432340 \quad 0.6138890 \quad 3.7492020$

$\begin{array}{llll}\mathrm{Si} & -0.4877400 & 0.7460450 & -3.1723140\end{array}$

$\begin{array}{llll}\text { C } & 0.4911070 & 1.1667480 & -4.6996180\end{array}$

$\begin{array}{llll}\text { C } & -2.1792370 & 0.0708500 & -3.5635750\end{array}$

$\begin{array}{llll}\text { C } & 2.6026640 & 2.2012050 & -0.1627740\end{array}$

C $\quad 1.6101940 \quad 2.3406940 \quad-1.3219520$

O $\quad 0.8641950 \quad-1.4292330 \quad 3.9563340$

C $\quad 0.6281120 \quad-1.3492260 \quad 5.3598200$ 


$\begin{array}{rrrr}\mathrm{H} & -1.4097520 & 1.1330210 & 1.5660800 \\ \mathrm{H} & -2.1788130 & 0.4336140 & 0.0968240 \\ \mathrm{H} & 1.3350120 & -1.4212850 & 1.6155990 \\ \mathrm{H} & -1.3968940 & -3.4060520 & -0.5258640 \\ \mathrm{H} & -0.0587160 & -3.1113130 & -1.6565960 \\ \mathrm{H} & 0.2633440 & -3.5111720 & 0.0441990 \\ \mathrm{H} & -3.3852940 & -0.8916910 & 1.7952900 \\ \mathrm{H} & -3.2915710 & -2.6240600 & 2.2072470 \\ \mathrm{H} & -3.0140320 & -2.0904560 & 0.5278020 \\ \mathrm{H} & 2.7098450 & 0.0294420 & -0.2090200 \\ \mathrm{H} & 3.1892810 & 0.7398450 & 1.3376390 \\ \mathrm{H} & 1.4703560 & 1.5853390 & -4.4494460 \\ \mathrm{H} & 0.6490120 & 0.2724400 & -5.3126300 \\ \mathrm{H} & -0.0449180 & 1.9018240 & -5.3098490 \\ \mathrm{H} & -2.8468140 & 0.8593680 & -3.9268530 \\ \mathrm{H} & -2.1293420 & -0.7138820 & -4.3259530 \\ \mathrm{H} & -2.6244520 & -0.3587220 & -2.6597560 \\ \mathrm{H} & 2.4210800 & 2.9830190 & 0.5875450 \\ \mathrm{H} & 3.6189420 & 2.3582560 & -0.5405100 \\ \mathrm{H} & 1.8996330 & 1.6133080 & -2.0884620 \\ \mathrm{H} & 1.6784890 & 3.3333030 & -1.7803920 \\ \mathrm{H} & 1.0221280 & -2.2751540 & 5.7781600 \\ \mathrm{H} & -0.4425350 & -1.2631950 & 5.5609590 \\ \mathrm{H} & 1.1410660 & -0.4851280 & 5.7897030\end{array}$

\section{Calculated endo transition state for entry 1:}

Single point: SCF $(\omega \mathrm{B} 97 \mathrm{xd} / 6-311 \mathrm{G}(\mathrm{d}, \mathrm{p}))=-1329.096869$

Sum of electronic and thermal Free Energies $(\omega \mathrm{B} 97 \mathrm{xd} / 6-31 \mathrm{G}(\mathrm{d}))=-1328.437906$ SCF $(\omega B 97 x d / 6-31 G(d))=-1328.800873$

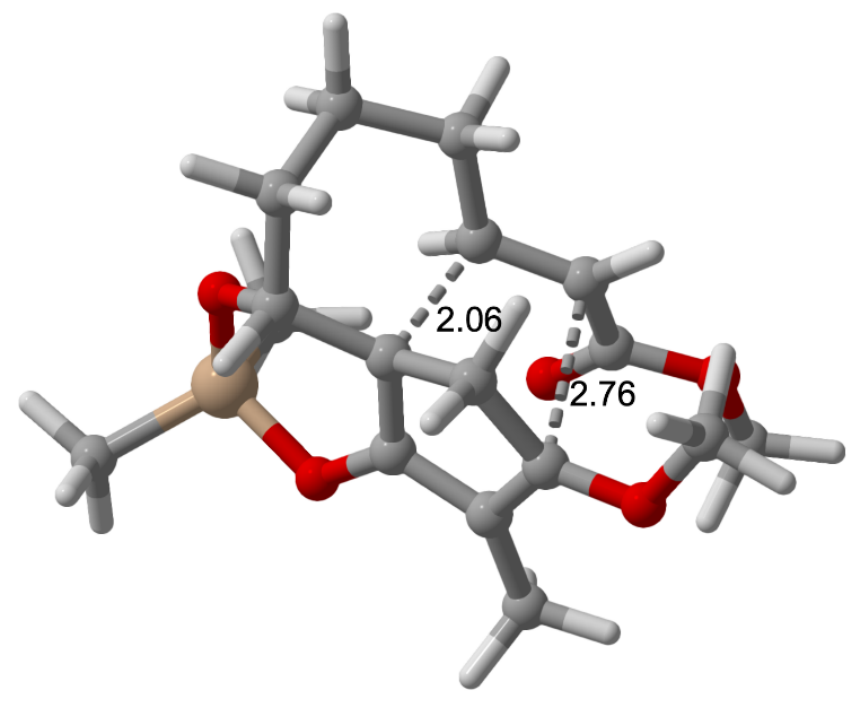

49 
E_17_endo_TS_M0003

$\begin{array}{lrrr}\mathrm{O} & -1.1019610 & 0.5612160 & -1.9100320 \\ \mathrm{C} & -1.1069800 & 0.1724200 & -0.6298090 \\ \mathrm{C} & -1.6424840 & -1.0646490 & -0.2204820 \\ \mathrm{C} & -1.5376460 & -1.0840670 & 1.1494900 \\ \mathrm{C} & -1.1780310 & 0.2811990 & 1.6757360 \\ \mathrm{C} & -0.5607460 & 0.9120770 & 0.4433740 \\ \mathrm{C} & -2.1029780 & -2.1589730 & -1.1245620 \\ \mathrm{C} & 1.2179640 & -1.1838250 & 1.0321400 \\ \mathrm{C} & 1.3415400 & 0.1680940 & 0.7092800 \\ \mathrm{C} & -0.4170020 & 2.4113400 & 0.2537640 \\ \mathrm{C} & 0.3830750 & 3.0960960 & 1.3539050 \\ \mathrm{O} & 0.2306230 & 2.6742650 & -0.9920770 \\ \mathrm{H} & -1.4196250 & 2.8698710 & 0.2195820 \\ \mathrm{H} & 1.6642950 & 0.3799660 & -0.3066780 \\ \mathrm{C} & 1.1468470 & -2.1572920 & -0.0194290 \\ \mathrm{O} & 1.1293150 & -1.9142380 & -1.2230920 \\ \mathrm{O} & -1.9632260 & -2.1125760 & 1.8871370 \\ \mathrm{C} & -1.6119970 & -2.1197350 & 3.2579800 \\ \mathrm{Si} & -0.0857320 & 1.8338590 & -2.3880560 \\ \mathrm{C} & 1.4852200 & 1.1222680 & -3.0814940 \\ \mathrm{C} & -1.0582340 & 2.8586990 & -3.5997380 \\ \mathrm{C} & 1.8227870 & 2.5966300 & 1.4299510 \\ \mathrm{C} & 1.9315270 & 1.1060090 & 1.7467460 \\ \mathrm{O} & 1.0798880 & -3.4325290 & 0.4501040 \\ \mathrm{C} & 0.9924180 & -4.4387410 & -0.5452880 \\ \mathrm{H} & -0.5342890 & 0.2680780 & 2.5562700 \\ \mathrm{H} & -2.1083690 & 0.8035090 & 1.9448830 \\ \mathrm{H} & -2.3356610 & -3.0586820 & -0.5494150 \\ \mathrm{H} & -1.3146580 & -2.3902010 & -1.8475900 \\ \mathrm{H} & -2.9985360 & -1.8621470 & -1.6810630 \\ \mathrm{H} & 1.2927570 & -1.5314140 & 2.0572840 \\ \mathrm{H} & 0.3692210 & 4.1751590 & 1.1645530 \\ \mathrm{H} & -0.1297130 & 2.9303330 & 2.3117760 \\ \mathrm{H} & -2.0469060 & -1.2632550 & 3.7866810 \\ \mathrm{H} & -0.5229220 & -2.1131490 & 3.3754410 \\ \mathrm{H} & -2.0195820 & -3.0432250 & 3.6691420 \\ \mathrm{H} & 2.3289060 & 1.8071940 & -2.9447690 \\ \mathrm{H} & 1.7069040 & 0.1769380 & -2.5723160 \\ \mathrm{H} & 1.3861970 & 0.9103820 & -4.1516340 \\ \mathrm{H} & -0.4604930 & 3.7013100 & -3.9641170 \\ \mathrm{H} & -1.9639570 & 3.2601560 & -3.1343470 \\ \mathrm{H} & -1.3572050 & 2.2580880 & -4.4657410 \\ \mathrm{H} & 2.3177360 & 2.8037600 & 0.4744030 \\ \mathrm{H} & 2.3619890 & 3.1654140 & 2.1964040 \\ \mathrm{H} & 2.9932920 & 0.8430730 & 1.8397260\end{array}$




$\begin{array}{llll}\mathrm{H} & 1.4900330 & 0.9012640 & 2.7324620 \\ \mathrm{H} & 1.8222220 & -4.3679430 & -1.2540350 \\ \mathrm{H} & 1.0313900 & -5.3894970 & -0.0111720 \\ \mathrm{H} & 0.0527870 & -4.3625300 & -1.1012760\end{array}$

Calculated exo transition state for entry 1:

Single point: SCF $(\omega \mathrm{B} 97 \mathrm{xd} / 6-311 \mathrm{G}(\mathrm{d}, \mathrm{p}))=-1329.081193$

Sum of electronic and thermal Free Energies $(\omega \mathrm{B} 97 \mathrm{xd} / 6-31 \mathrm{G}(\mathrm{d}))=-1328.423497$ $\operatorname{SCF}(\omega \mathrm{B} 97 \mathrm{xd} / 6-31 \mathrm{G}(\mathrm{d}))=-1328.78625$

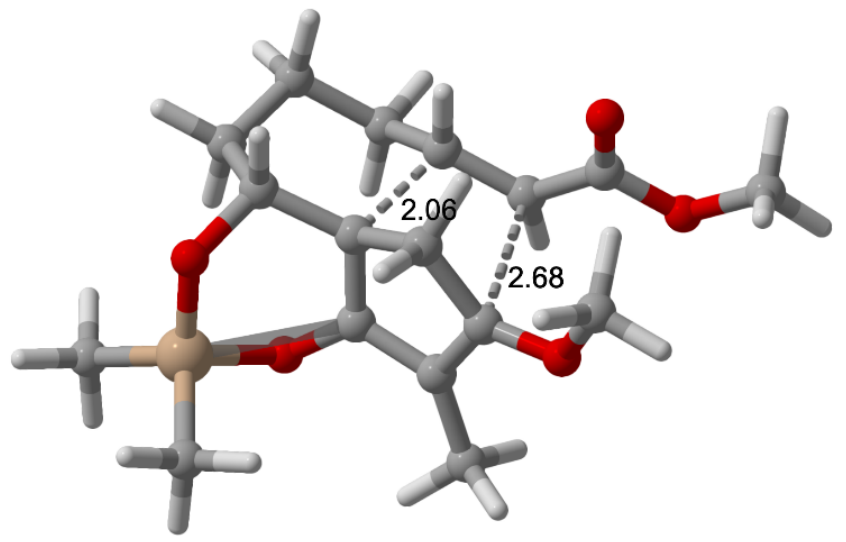

49

E_17_exo_TS_M0001

$\mathrm{O}-0.5 \overline{2} 47 \overline{7} 20 \quad-0.4435420 \quad-2.2667610$

$\begin{array}{llll}\text { C } & 0.0302830 & -0.4270930 & -1.0039080\end{array}$

$\begin{array}{llll}\text { C } & -0.4737610 & -1.5521800 & -0.3347290\end{array}$

$\begin{array}{llll}\text { C } & -1.1367250 & -1.0576180 & 0.7769050\end{array}$

$\begin{array}{llll}\text { C } & -1.2444020 & 0.4393830 & 0.7078440\end{array}$

$\begin{array}{llll}\text { C } & -0.1175040 & 0.7632280 & -0.2611620\end{array}$

$\begin{array}{llll}\text { C } & -0.0297020 & 2.0498680 & -1.0603380\end{array}$

$\begin{array}{llll}\text { C } & 1.3293930 & 0.7626900 & 1.2109370\end{array}$

$\begin{array}{llll}\mathrm{C} & 1.2310970 & -0.4522900 & 1.8869850\end{array}$

$\begin{array}{llll}\text { C } & -0.3424400 & -2.9828520 & -0.7413030\end{array}$

$\begin{array}{llll}\mathrm{O} & -1.8188080 & -1.8520750 & 1.5977810\end{array}$

$\begin{array}{llll}\text { C } & -2.7017780 & -1.2403100 & 2.5353710\end{array}$

$\begin{array}{llll}\mathrm{O} & -0.7573800 & 1.9007990 & -2.3004300\end{array}$

$\begin{array}{llll}\mathrm{H} & -0.5274360 & 2.8562240 & -0.5109460\end{array}$

$\begin{array}{llll}\mathrm{C} & 2.5587500 & 1.1677490 & 0.4315650\end{array}$

$\begin{array}{llll}\mathrm{H} & 0.8977950 & 1.5988810 & 1.7647480\end{array}$

$\begin{array}{llll}\text { C } & 0.5666440 & -0.5349040 & 3.1614750\end{array}$

$\begin{array}{llll}\mathrm{O} & -0.0953530 & 0.3437630 & 3.7099600\end{array}$

$\begin{array}{llll}\mathrm{Si} & -0.3912630 & 0.5968770 & -3.2677410\end{array}$

$\begin{array}{llll}\mathrm{C} & 0.6680140 & 1.0013660 & -4.7440620\end{array}$

$\begin{array}{llll}\text { C } & -1.9795110 & -0.2570570 & -3.7346270\end{array}$

$\begin{array}{llll}\text { C } & 2.3822160 & 2.5323320 & -0.2357670\end{array}$ 


$\begin{array}{rrrr}\mathrm{C} & 1.4040760 & 2.4837630 & -1.4045140 \\ \mathrm{O} & 0.7393140 & -1.7511270 & 3.7408930 \\ \mathrm{C} & 0.1418050 & -1.9100120 & 5.0167750 \\ \mathrm{H} & -1.1976810 & 0.9307410 & 1.6813940 \\ \mathrm{H} & -2.2025510 & 0.6915970 & 0.2283550 \\ \mathrm{H} & 1.7879830 & -1.3236800 & 1.5593560 \\ \mathrm{H} & -1.2666670 & -3.5341140 & -0.5433160 \\ \mathrm{H} & -0.1067250 & -3.0530980 & -1.8067370 \\ \mathrm{H} & 0.4611940 & -3.4775170 & -0.1839290 \\ \mathrm{H} & -2.1564410 & -0.5770260 & 3.2104120 \\ \mathrm{H} & -3.1484680 & -2.0623790 & 3.0949690 \\ \mathrm{H} & -3.4901960 & -0.6856350 & 2.0128920 \\ \mathrm{H} & 2.8189740 & 0.4035460 & -0.3124050 \\ \mathrm{H} & 3.3972730 & 1.2189460 & 1.1378760 \\ \mathrm{H} & 1.5930900 & 1.5090690 & -4.4566630 \\ \mathrm{H} & 0.9344350 & 0.0861600 & -5.2843760 \\ \mathrm{H} & 0.1256760 & 1.6540630 & -5.4368030 \\ \mathrm{H} & -2.6724990 & 0.4342310 & -4.2258760 \\ \mathrm{H} & -1.7964670 & -1.0969940 & -4.4132670 \\ \mathrm{H} & -2.4720030 & -0.6445980 & -2.8362180 \\ \mathrm{H} & 2.0496990 & 3.2610770 & 0.5165360 \\ \mathrm{H} & 3.3481620 & 2.8918160 & -0.6083780 \\ \mathrm{H} & 1.8120440 & 1.7780780 & -2.1365480 \\ \mathrm{H} & 1.3427270 & 3.4570070 & -1.9042000 \\ \mathrm{H} & 0.4503610 & -2.8957880 & 5.3675990 \\ \mathrm{H} & -0.9501670 & -1.8651600 & 4.9531520 \\ \mathrm{H} & 0.4801520 & -1.1362080 & 5.7112270\end{array}$

Calculated silacycle diene for entry 2:

Single point: SCF $(\omega \mathrm{B} 97 \mathrm{xd} / 6-311 \mathrm{G}(\mathrm{d}, \mathrm{p}))=-1329.115937$

Sum of electronic and thermal Free Energies $(\omega \mathrm{B} 97 \mathrm{xd} / 6-31 \mathrm{G}(\mathrm{d}))=-1328.460284$ $\operatorname{SCF}(\omega \mathrm{B} 97 \mathrm{xd} / 6-31 \mathrm{G}(\mathrm{d}))=-1328.819137$

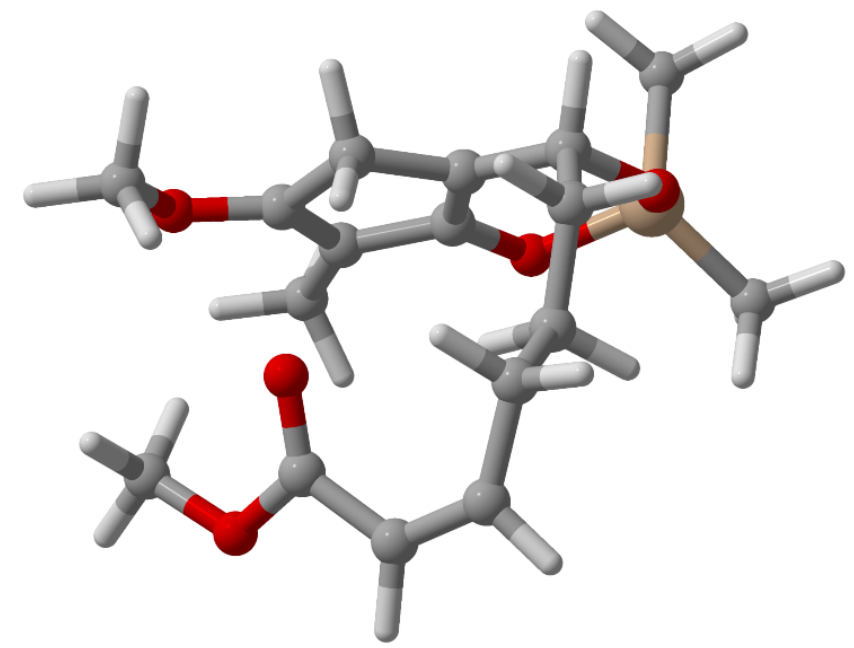


49

E $30 \mathrm{sm} \mathrm{M0001}$

$\mathrm{C}^{-} \quad 0 . \overline{4488460} \quad 1.4883190 \quad 0.0004350$

$\begin{array}{llll}\mathrm{C} & 0.4855460 & 1.5812500 & 1.4390730\end{array}$

$\begin{array}{llll}\mathrm{C} & 0.9619730 & 0.4434420 & 1.9813260\end{array}$

$\begin{array}{llll}\text { C } & 1.5677020 & -0.4537070 & 0.9303980\end{array}$

$\begin{array}{llll}\text { C } & 1.3768330 & 0.3387910 & -0.3334620\end{array}$

$\begin{array}{llll}\mathrm{O} & 0.3476250 & 2.4791690 & -0.8395580\end{array}$

C $\quad-0.1892870 \quad 2.7311680 \quad 2.1110920$

$\begin{array}{llll}\text { O } & 0.9231900 & 0.1572260 & 3.3038250\end{array}$

C $\quad 1.2481330 \quad-1.1700930 \quad 3.6770360$

$\begin{array}{llll}\text { C } & 1.6317090 & -0.1771180 & -1.7238810\end{array}$

$\begin{array}{llll}\text { C } & 0.9567520 & -1.5357290 & -1.9570290\end{array}$

C $\quad-0.5523110 \quad-1.4877660 \quad-1.7204740$

$\begin{array}{llll}\text { C } & -1.1718720 & -2.8745990 & -1.4731810\end{array}$

$\begin{array}{llll}\text { C } & -2.6207150 & -2.7452650 & -1.0960090\end{array}$

C $\quad-3.1133650 \quad-2.3720230 \quad 0.0926050$

C $\quad-2.2926730 \quad-2.0635380 \quad 1.2825950$

O $\quad-1.1066550 \quad-2.2925970 \quad 1.4304220$

O $\quad-3.0407230 \quad-1.4732540 \quad 2.2266150$

$\begin{array}{llll}\mathrm{O} & 1.1326630 & 0.7342630 & -2.7069640\end{array}$

$\begin{array}{llll}\mathrm{Si} & 0.9795100 & 2.3552010 & -2.4013250\end{array}$

$\begin{array}{llll}\text { C } & 2.6371560 & 3.2135440 & -2.4196650\end{array}$

C $\quad-0.2546160 \quad 3.0725700 \quad-3.5894390$

C $\quad-2.3472920 \quad-1.0729610 \quad 3.4078710$

$\mathrm{H} \quad 2.6243150 \quad-0.6667150 \quad 1.1464640$

$\mathrm{H} \quad 1.0359470 \quad-1.4132320 \quad 0.8965780$

$\mathrm{H} \quad 0.3914040 \quad 3.6542940 \quad 1.9995820$

$\mathrm{H} \quad-0.3131970 \quad 2.5319910 \quad 3.1789420$

$\mathrm{H} \quad-1.1765380 \quad 2.9196760 \quad 1.6741060$

$\mathrm{H} \quad 0.5852780 \quad-1.8897750 \quad 3.1845630$

$\mathrm{H} \quad 2.2923810 \quad-1.4073920 \quad 3.4376910$

$\mathrm{H} \quad 1.1133850 \quad-1.2196720 \quad 4.7589130$

$\mathrm{H} \quad 2.7118200 \quad-0.3035960 \quad-1.9011710$

$\mathrm{H} \quad 1.4201350 \quad-2.2610960 \quad-1.2749080$

$\mathrm{H} \quad 1.1837060 \quad-1.8683670 \quad-2.9767140$

$\mathrm{H} \quad-0.7518500 \quad-0.8677960 \quad-0.8405320$

$\mathrm{H} \quad-1.0437480 \quad-0.9960110 \quad-2.5669890$

$\mathrm{H} \quad-0.6232380 \quad-3.3617240 \quad-0.6627340$

$\mathrm{H} \quad-1.0780880 \quad-3.4967350 \quad-2.3705740$

$\mathrm{H} \quad-3.3472410 \quad-2.9102330 \quad-1.8907030$

$\mathrm{H} \quad-4.1829850 \quad-2.2437810 \quad 0.2239820$

$\mathrm{H} \quad 3.2931160 \quad 2.7792990 \quad-1.6572400$

$\mathrm{H} \quad 2.5342340 \quad 4.2825270 \quad-2.2043760$

$\mathrm{H} \quad 3.1300440 \quad 3.1061000 \quad-3.3919340$

$\mathrm{H} \quad 0.1084930 \quad 3.0080410 \quad-4.6205030$ 


$\begin{array}{lrrr}\mathrm{H} & -1.2029970 & 2.5305620 & -3.5262190 \\ \mathrm{H} & -0.4469680 & 4.1267590 & -3.3632540 \\ \mathrm{H} & -1.5405000 & -0.3753910 & 3.1689030 \\ \mathrm{H} & -3.0958970 & -0.5901840 & 4.0354140 \\ \mathrm{H} & -1.9291410 & -1.9438420 & 3.9204110\end{array}$

\section{Calculated endo product for entry 2:}

Single point: SCF $(\omega \mathrm{B} 97 \mathrm{xd} / 6-311 \mathrm{G}(\mathrm{d}, \mathrm{p}))=-1329.143777$

Sum of electronic and thermal Free Energies $(\omega \mathrm{B} 97 \mathrm{xd} / 6-31 \mathrm{G}(\mathrm{d}))=-1328.483275$ $\mathrm{SCF}(\omega \mathrm{B} 97 \mathrm{xd} / 6-31 \mathrm{G}(\mathrm{d}))=-1328.851295$

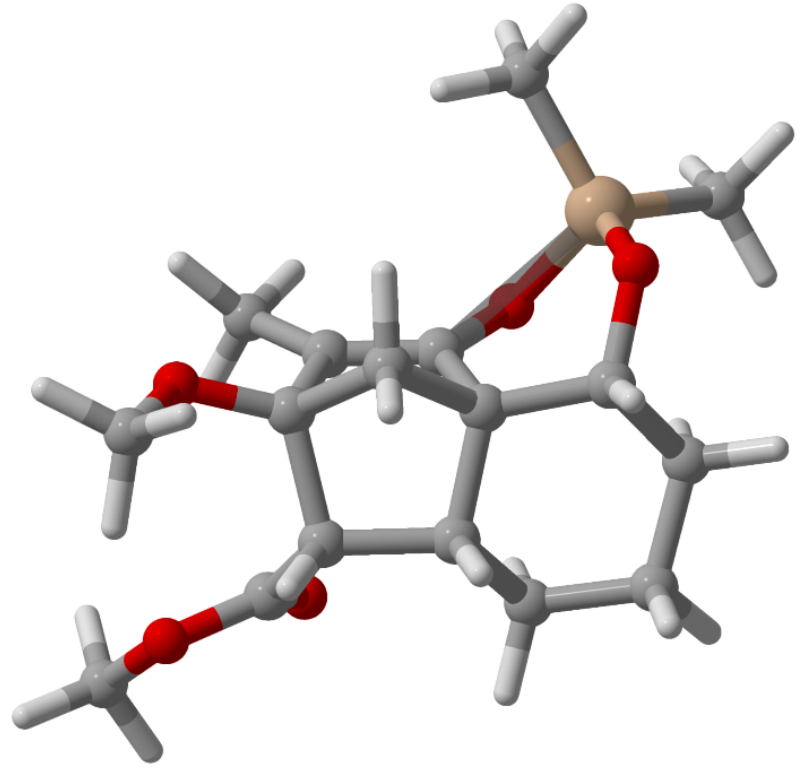

49

E_30_endo_M0002

$\begin{array}{lrrr}\mathrm{O} & 0.1089460 & 0.0062980 & -2.1865630 \\ \mathrm{C} & -0.2640760 & -0.1195080 & -0.8810330 \\ \mathrm{C} & -0.7766280 & -1.1800440 & -0.2553630 \\ \mathrm{C} & -1.0637250 & -0.7022150 & 1.1615230 \\ \mathrm{C} & -1.4545840 & 0.7793040 & 0.8985240 \\ \mathrm{C} & -0.1721330 & 1.0525180 & 0.0894690 \\ \mathrm{C} & 0.0856280 & 2.3829900 & -0.6154180 \\ \mathrm{C} & 0.8895000 & 0.7520500 & 1.1996850 \\ \mathrm{C} & 0.3187340 & -0.5010550 & 1.9159780 \\ \mathrm{C} & -0.9863670 & -2.5722040 & -0.7404240 \\ \mathrm{O} & -1.9825810 & -1.5521470 & 1.7724070 \\ \mathrm{C} & -2.3542510 & -1.2347660 & 3.0923910 \\ \mathrm{O} & -0.7422310 & 2.4955770 & -1.7735820 \\ \mathrm{H} & -0.1989130 & 3.2061790 & 0.0515640 \\ \mathrm{C} & 2.3159710 & 0.7967250 & 0.6706850 \\ \mathrm{H} & 0.8075080 & 1.5769000 & 1.9227920 \\ \mathrm{Si} & -0.6907350 & 1.2984520 & -2.9341870\end{array}$




$\begin{array}{lrrr}\mathrm{C} & 0.3020690 & 1.7597790 & -4.4408020 \\ \mathrm{C} & -2.4422400 & 0.8166810 & -3.3457050 \\ \mathrm{C} & 2.5555510 & 2.2086250 & 0.1227880 \\ \mathrm{C} & 1.5699770 & 2.5380150 & -1.0028450 \\ \mathrm{H} & -1.5348390 & 1.3970100 & 1.8021170 \\ \mathrm{H} & -2.3719760 & 0.8514810 & 0.3097680 \\ \mathrm{H} & -2.0000710 & -2.9180030 & -0.5153530 \\ \mathrm{H} & -0.8196000 & -2.6226250 & -1.8197990 \\ \mathrm{H} & -0.2850510 & -3.2632890 & -0.2579470 \\ \mathrm{H} & -1.5460640 & -1.4453370 & 3.8040860 \\ \mathrm{H} & -3.2078670 & -1.8718650 & 3.3330890 \\ \mathrm{H} & -2.6610060 & -0.1844970 & 3.1927470 \\ \mathrm{H} & 2.4597260 & 0.0454660 & -0.1122340 \\ \mathrm{H} & 3.0320740 & 0.5677250 & 1.4678980 \\ \mathrm{H} & 1.3187750 & 2.0652980 & -4.1768850 \\ \mathrm{H} & 0.3698610 & 0.9101590 & -5.1292750 \\ \mathrm{H} & -0.1726920 & 2.5892430 & -4.9762110 \\ \mathrm{H} & -3.0394820 & 1.6907660 & -3.6263740 \\ \mathrm{H} & -2.4736300 & 0.1004000 & -4.1736610 \\ \mathrm{H} & -2.9133560 & 0.3504140 & -2.4738490 \\ \mathrm{H} & 2.4556880 & 2.9383020 & 0.9391760 \\ \mathrm{H} & 3.5780620 & 2.3044100 & -0.2585190 \\ \mathrm{H} & 1.7829030 & 1.8545960 & -1.8319750 \\ \mathrm{H} & 1.7235600 & 3.5559180 & -1.3777530 \\ \mathrm{C} & 1.1333180 & -1.7658720 & 1.9015540 \\ \mathrm{O} & 1.8855370 & -2.1396740 & 1.0317280 \\ \mathrm{O} & 0.8743440 & -2.4965020 & 3.0044010 \\ \mathrm{C} & 1.5047960 & -3.7738880 & 3.0485310 \\ \mathrm{H} & 1.1790460 & -4.3910360 & 2.2070880 \\ \mathrm{H} & 1.1956670 & -4.2232870 & 3.9919740 \\ \mathrm{H} & 2.5919940 & -3.6698260 & 3.0108300 \\ \mathrm{H} & 0.1148610 & -0.2636380 & 2.9629650\end{array}$

\section{Calculated exo product for entry 2:}

Single point: SCF $(\omega B 97 x d / 6-311 G(d, p))=-1329.153024$

Sum of electronic and thermal Free Energies $(\omega \mathrm{B} 97 \mathrm{xd} / 6-31 \mathrm{G}(\mathrm{d}))=-1328.492539$ SCF $(\omega B 97 x d / 6-31 G(d))=-1328.85968$ 


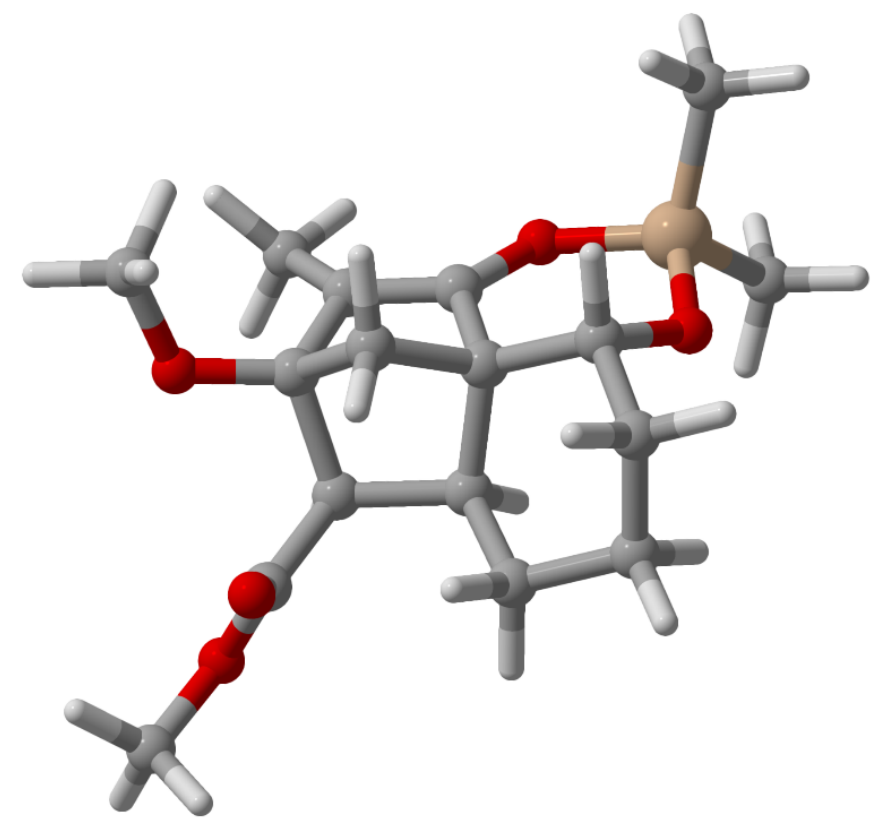

49

E_30_exo_M0001

$\begin{array}{lrrr}\mathrm{O}^{-} & -2.2506930 & -1.2580540 & -0.3604150 \\ \mathrm{C} & -1.1024750 & -0.9006020 & 0.2664610 \\ \mathrm{C} & -0.2511240 & -1.7205020 & 0.8896050 \\ \mathrm{C} & 0.9725660 & -0.8658490 & 1.1925340 \\ \mathrm{C} & 0.3374820 & 0.5136020 & 1.4575690 \\ \mathrm{C} & -0.4732280 & 0.4894700 & 0.1515350 \\ \mathrm{C} & -0.2810780 & -3.2064110 & 1.0082060 \\ \mathrm{C} & 1.6793160 & -0.6256790 & -0.1859280 \\ \mathrm{C} & 0.6808200 & 0.3448410 & -0.9131400 \\ \mathrm{C} & -1.4599970 & 1.6292290 & -0.1081120 \\ \mathrm{C} & -0.7707120 & 2.9220550 & -0.5249320 \\ \mathrm{O} & -2.3564860 & 1.2682770 & -1.1644930 \\ \mathrm{H} & -2.0430980 & 1.8049490 & 0.8099020 \\ \mathrm{H} & 0.2827770 & -0.1521990 & -1.8043400 \\ \mathrm{O} & 1.8835210 & -1.4082590 & 2.1032470 \\ \mathrm{C} & 1.4612060 & -1.3701800 & 3.4450650 \\ \mathrm{Si} & -3.2790350 & -0.0987700 & -1.0153900 \\ \mathrm{C} & -3.8129490 & -0.6517230 & -2.7059390 \\ \mathrm{C} & -4.7035530 & 0.1542770 & 0.1637380 \\ \mathrm{C} & 0.1546070 & 2.6903190 & -1.7194770 \\ \mathrm{C} & 1.2576360 & 1.6995250 & -1.3477880 \\ \mathrm{H} & 1.0776130 & 1.3160200 & 1.5146320 \\ \mathrm{H} & -0.3057090 & 0.5208650 & 2.3423390 \\ \mathrm{H} & 0.5642650 & -3.6542030 & 0.4697860 \\ \mathrm{H} & -1.2078460 & -3.6069520 & 0.5887500 \\ \mathrm{H} & -0.2001150 & -3.5385720 & 2.0497540 \\ \mathrm{H} & -1.5431430 & 3.6615840 & -0.7602420 \\ & & & \end{array}$




$\begin{array}{lrrr}\mathrm{H} & -0.1923930 & 3.3065820 & 0.3254670 \\ \mathrm{H} & 0.4544460 & -1.7940110 & 3.5692980 \\ \mathrm{H} & 1.4614050 & -0.3447050 & 3.8383110 \\ \mathrm{H} & 2.1735510 & -1.9691420 & 4.0162310 \\ \mathrm{H} & -4.4747760 & 0.0879660 & -3.1688420 \\ \mathrm{H} & -2.9437670 & -0.7863440 & -3.3568610 \\ \mathrm{H} & -4.3524650 & -1.6034090 & -2.6549500 \\ \mathrm{H} & -5.4052620 & 0.9031450 & -0.2192400 \\ \mathrm{H} & -4.3471670 & 0.4942400 & 1.1422900 \\ \mathrm{H} & -5.2542300 & -0.7799070 & 0.3187160 \\ \mathrm{H} & -0.4355960 & 2.3146540 & -2.5636440 \\ \mathrm{H} & 0.6015360 & 3.6395660 & -2.0346370 \\ \mathrm{H} & 1.9388200 & 1.5440370 & -2.1938380 \\ \mathrm{H} & 1.8586780 & 2.1306160 & -0.5416780 \\ \mathrm{H} & 1.7704910 & -1.5713450 & -0.7228560 \\ \mathrm{C} & 3.0786170 & -0.0887330 & 0.0232390 \\ \mathrm{O} & 3.3840990 & 0.8801740 & 0.6821810 \\ \mathrm{O} & 3.9855420 & -0.8431380 & -0.6190050 \\ \mathrm{C} & 5.3415980 & -0.4377580 & -0.4476890 \\ \mathrm{H} & 5.6206720 & -0.4733980 & 0.6081940 \\ \mathrm{H} & 5.4897370 & 0.5796540 & -0.8187130 \\ \mathrm{H} & 5.9358950 & -1.1458040 & -1.0248990\end{array}$

Calculated endo transition state for entry 2:

Single point: SCF $(\omega B 97 x d / 6-311 G(d, p))=-1329.079175$

Sum of electronic and thermal Free Energies $(\omega \mathrm{B} 97 \mathrm{xd} / 6-31 \mathrm{G}(\mathrm{d}))=-1328.4356$ $\operatorname{SCF}(\omega \mathrm{B} 97 \mathrm{xd} / 6-31 \mathrm{G}(\mathrm{d}))=-1328.796622$

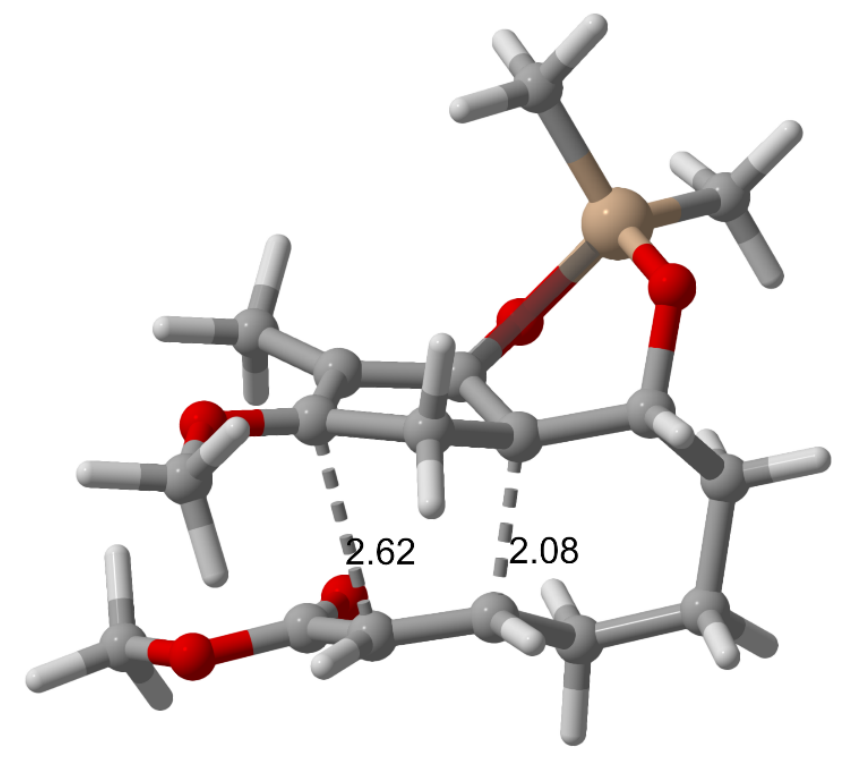

49

E_30_endo_TS_M0002 


$\begin{array}{lrrr}\mathrm{O} & 0.1405600 & -0.0946900 & -1.9848070 \\ \mathrm{C} & -0.2961770 & -0.1008210 & -0.7033500 \\ \mathrm{C} & -0.9289240 & -1.1948400 & -0.0882740 \\ \mathrm{C} & -1.4488450 & -0.7070530 & 1.0943390 \\ \mathrm{C} & -1.3978900 & 0.8010840 & 1.0991830 \\ \mathrm{C} & -0.2643030 & 1.0440650 & 0.1160410 \\ \mathrm{C} & -0.0046690 & 2.3579760 & -0.5946090 \\ \mathrm{C} & 1.2074150 & 0.7707750 & 1.5637740 \\ \mathrm{C} & 0.8878660 & -0.4084880 & 2.2356770 \\ \mathrm{C} & -0.9570450 & -2.5967940 & -0.5982580 \\ \mathrm{O} & -2.1839340 & -1.4666140 & 1.9121330 \\ \mathrm{C} & -2.6444110 & -0.8783510 & 3.1130050 \\ \mathrm{O} & -0.7777590 & 2.4088550 & -1.8138850 \\ \mathrm{H} & -0.3599930 & 3.1885690 & 0.0255240 \\ \mathrm{C} & 2.4646510 & 1.0117540 & 0.7585720 \\ \mathrm{H} & 0.9200450 & 1.6666940 & 2.1205330 \\ \mathrm{Si} & -0.6349570 & 1.1355560 & -2.8784190 \\ \mathrm{C} & 0.4319150 & 1.4852730 & -4.3626530 \\ \mathrm{C} & -2.3466090 & 0.5620740 & -3.3398560 \\ \mathrm{C} & 2.4777460 & 2.4252540 & 0.1754470 \\ \mathrm{C} & 1.4708900 & 2.5933610 & -0.9587540 \\ \mathrm{H} & 1.3364670 & -3.9995120 & 2.3321200 \\ \mathrm{H} & -1.2739940 & 1.2556050 & 2.0838470 \\ \mathrm{H} & -2.3361640 & 1.1744540 & 0.6619360 \\ \mathrm{H} & -1.6189050 & -3.2197870 & 0.0082070 \\ \mathrm{H} & -1.3011620 & -2.6234300 & -1.6375520 \\ \mathrm{H} & 0.0539690 & -3.0175260 & -0.5705840 \\ \mathrm{H} & -1.8041770 & -0.5683840 & 3.7436280 \\ \mathrm{H} & -3.2180100 & -1.6499480 & 3.6264090 \\ \mathrm{H} & -3.2913990 & -0.0160180 & 2.9121720 \\ \mathrm{H} & 2.5739210 & 0.2553670 & -0.0226200 \\ \mathrm{H} & 3.3210040 & 0.8877430 & 1.4339190 \\ \mathrm{H} & 1.4279370 & 1.8332180 & -4.0745740 \\ \mathrm{H} & 0.5494170 & 0.5795130 & -4.9678220 \\ \mathrm{H} & -0.0265580 & 2.2544700 & -4.9936220 \\ \mathrm{H} & -2.9419570 & 1.3805560 & -3.7583290 \\ \mathrm{H} & -2.8664750 & 0.1887800 & -2.4507300 \\ \mathrm{H} & 1.354830600 & -4.3186930 & 1.4201210\end{array}$




$\begin{array}{llll}\mathrm{H} & 1.0349070 & -4.6321040 & 3.1683540 \\ \mathrm{H} & 2.4148550 & -4.0749520 & 2.1679290 \\ \mathrm{H} & 0.3463010 & -0.3673230 & 3.1735360\end{array}$

Calculated exo transition state for entry 2:

Single point: SCF $(\omega \mathrm{B} 97 \mathrm{xd} / 6-311 \mathrm{G}(\mathrm{d}, \mathrm{p}))=-1329.092356$

Sum of electronic and thermal Free Energies $(\omega \mathrm{B} 97 \mathrm{xd} / 6-31 \mathrm{G}(\mathrm{d}))=-1328.420758$ $\operatorname{SCF}(\omega \mathrm{B} 97 \mathrm{xd} / 6-31 \mathrm{G}(\mathrm{d}))=-1328.783869$

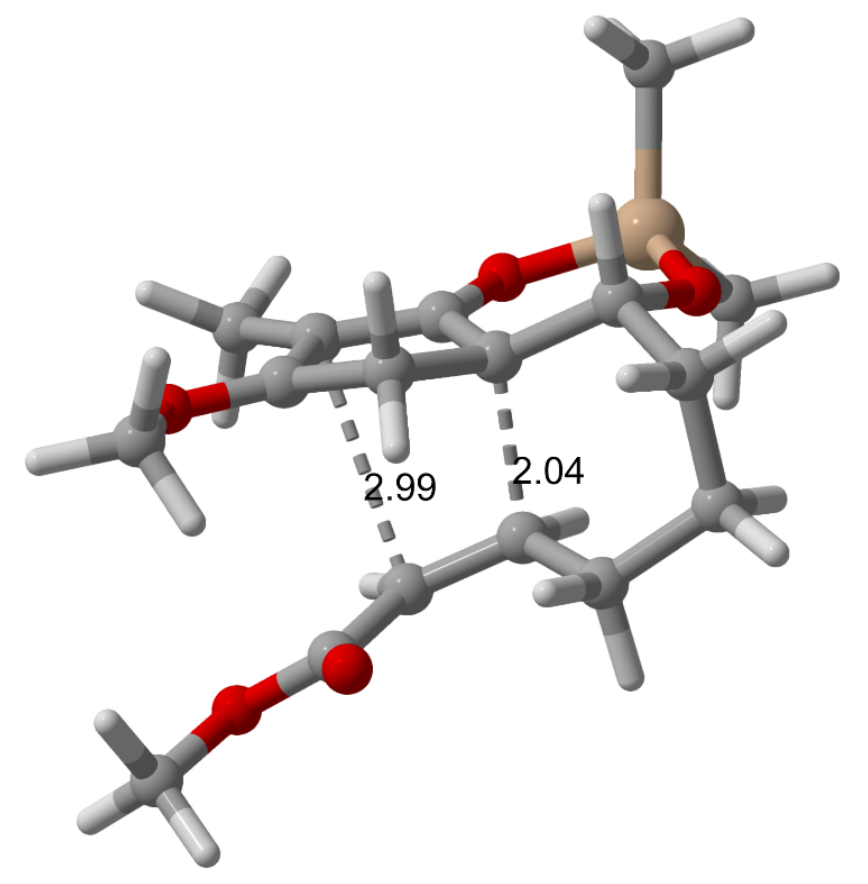

49

E_30_exo_TS_M0001

$\mathrm{O}^{-}{ }^{-}-2.1 \overline{5} 90 \overline{8} 40 \quad-0.5093850 \quad 1.2923000$

$\begin{array}{llll}\text { C } & -1.1294320 & 0.2489760 & 0.8743490\end{array}$

$\begin{array}{llll}\text { C } & -0.2954190 & 0.9289580 & 1.7753280\end{array}$

$\begin{array}{llll}\text { C } & 0.6573760 & 1.5682090 & 1.0068030\end{array}$

$\begin{array}{llll}\text { C } & 0.3234870 & 1.4755960 & -0.4534130\end{array}$

$\begin{array}{llll}\text { C } & -0.6547260 & 0.3184250 & -0.4579870\end{array}$

$\begin{array}{llll}\text { C } & -0.3554860 & 0.8781090 & 3.2666590\end{array}$

$\begin{array}{llll}\text { C } & 1.6869870 & -0.8934430 & 0.4843250\end{array}$

$\begin{array}{llll}\text { C } & 0.7071860 & -1.1984160 & -0.4772110\end{array}$

$\begin{array}{llll}\text { C } & -1.6318900 & 0.1090400 & -1.6029870\end{array}$

$\begin{array}{llll}\text { C } & -0.9663930 & -0.1789140 & -2.9408340\end{array}$

$\begin{array}{llll}\mathrm{O} & -2.4989090 & -0.9888850 & -1.3036010\end{array}$

$\begin{array}{llll}\mathrm{H} & -2.2512720 & 1.0155930 & -1.7153680\end{array}$

$\mathrm{H} \quad-0.0038380 \quad-1.9645790 \quad-0.1632600$

$\begin{array}{llll}\mathrm{O} & 1.5930150 & 2.3458480 & 1.5429840\end{array}$

$\begin{array}{llll}\text { C } & 2.4330000 & 3.0839380 & 0.6592720\end{array}$ 


$\begin{array}{lrrr}\mathrm{Si} & -3.2632490 & -1.1139640 & 0.1557960 \\ \mathrm{C} & -3.5720900 & -2.9049320 & 0.5304320 \\ \mathrm{C} & -4.7907020 & -0.0486680 & 0.2410000 \\ \mathrm{C} & -0.1122740 & -1.4423300 & -2.9053850 \\ \mathrm{C} & 1.0719400 & -1.3247290 & -1.9492760 \\ \mathrm{H} & 1.2022290 & 1.3349270 & -1.0869310 \\ \mathrm{H} & -0.1807270 & 2.4034540 & -0.7624730 \\ \mathrm{H} & 0.3678160 & 0.1537530 & 3.6597040 \\ \mathrm{H} & -1.3513910 & 0.5749530 & 3.5995940 \\ \mathrm{H} & -0.1158720 & 1.8520030 & 3.7037770 \\ \mathrm{H} & -1.7546470 & -0.2683480 & -3.6967000 \\ \mathrm{H} & -0.3482020 & 0.6864060 & -3.2166450 \\ \mathrm{H} & 1.8427850 & 3.8038630 & 0.0799390 \\ \mathrm{H} & 2.9774400 & 2.4158270 & -0.0124130 \\ \mathrm{H} & 3.1309140 & 3.6223470 & 1.3006150 \\ \mathrm{H} & -4.2635550 & -3.3430650 & -0.1970400 \\ \mathrm{H} & -2.6353150 & -3.4693080 & 0.4919850 \\ \mathrm{H} & -4.0062890 & -3.0294080 & 1.5280260 \\ \mathrm{H} & -5.5485680 & -0.3892410 & -0.4727500 \\ \mathrm{H} & -4.5496300 & 0.9937080 & 0.0053150 \\ \mathrm{H} & -5.2327060 & -0.0703060 & 1.2429830 \\ \mathrm{H} & -0.7459760 & -2.2924910 & -2.6260890 \\ \mathrm{H} & 0.2659710 & -1.6470720 & -3.9135750 \\ \mathrm{H} & 1.6906220 & -2.2278990 & -2.0344930 \\ \mathrm{H} & 1.7222300 & -0.4987700 & -2.2461970 \\ \mathrm{H} & 1.5373280 & -1.2168060 & 1.5075660 \\ \mathrm{C} & 2.9562260 & -0.2792900 & 0.2169590 \\ \mathrm{O} & 3.3485650 & 0.2554710 & -0.8213840 \\ \mathrm{O} & 3.7560510 & -0.3089020 & 1.3211100 \\ \mathrm{C} & 5.0367330 & 0.2743920 & 1.1615170 \\ \mathrm{H} & 4.9665120 & 1.3517770 & 0.9777420 \\ \mathrm{H} & 5.5804530 & -0.1815700 & 0.3294160 \\ \mathrm{H} & 5.5627750 & 0.0951470 & 2.1005180\end{array}$

Calculated silacycle diene for entry 3:

Single point: SCF $(\omega \mathrm{B} 97 \mathrm{xd} / 6-311 \mathrm{G}(\mathrm{d}, \mathrm{p}))=-1289.806162$

Sum of electronic and thermal Free Energies $(\omega \mathrm{B} 97 \mathrm{xd} / 6-31 \mathrm{G}(\mathrm{d}))=-1289.185626$ SCF $(\omega B 97 x d / 6-31 G(d))=-1289.517246$ 


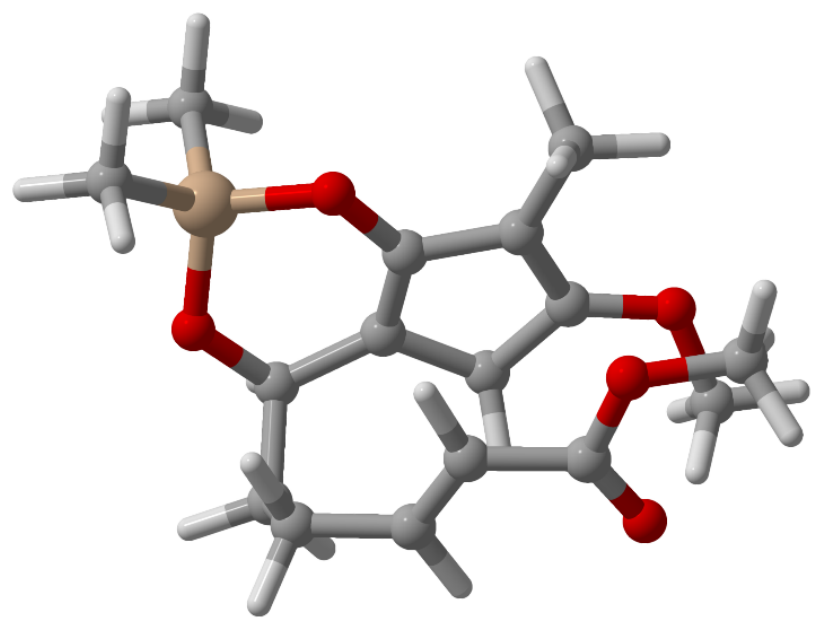

46

E_ester_4_sm_cs_dft_M0001

$\begin{array}{llll}\mathrm{C}^{-} & -0.0 \overline{1} 86 \overline{530} & -1.0968000 & -0.0269590 \\ \mathrm{C} & 1.2686890 & -0.8863490 & 0.6350120\end{array}$

$\begin{array}{llll}\text { C } & 1.2686890 & -0.8863490 & 0.6350120\end{array}$

$\begin{array}{llll}\mathrm{C} & 1.0167880 & -0.1159380 & 1.7120960\end{array}$

$\begin{array}{llll}\text { C } & -1.0353150 & -0.4756990 & 0.6110890\end{array}$

$\begin{array}{llll}\mathrm{O} & 1.9545400 & 0.2671330 & 2.6061110\end{array}$

C $\quad 1.6111910 \quad 1.3304240 \quad 3.4798340$

$\mathrm{H} \quad 1.3925220 \quad 2.2423640 \quad 2.9155490$

$\mathrm{H} \quad 2.4857050 \quad 1.4921230 \quad 4.1116130$

$\mathrm{H} \quad 0.7576090 \quad 1.0632360 \quad 4.1154210$

O $\quad-0.0897730 \quad-1.8146400 \quad-1.1797500$

C $\quad-2.4469480 \quad-0.3279460 \quad 0.1147480$

$\mathrm{H} \quad-3.1558770 \quad-0.7140830 \quad 0.8647100$

O $\quad-2.6492110 \quad-1.0675400 \quad-1.0908520$

$\begin{array}{llll}\mathrm{Si} & -1.6576850 & -2.3052040 & -1.5716030\end{array}$

C $\quad-1.7698050 \quad-2.4734780 \quad-3.4176480$

$\mathrm{H} \quad-1.0734170 \quad-3.2355190 \quad-3.7833530$

$\mathrm{H} \quad-1.5239830 \quad-1.5247640 \quad-3.9045090$

$\mathrm{H} \quad-2.7801380 \quad-2.7628090 \quad-3.7251980$

C $\quad-2.0250500 \quad-3.8810650 \quad-0.6427090$

$\mathrm{H} \quad-1.3677020 \quad-4.6953470 \quad-0.9659890$

$\mathrm{H} \quad-3.0623330 \quad-4.1985120 \quad-0.7942310$

$\mathrm{H} \quad-1.8705640 \quad-3.7295070 \quad 0.4310580$

C $\quad-2.8610420 \quad 1.1240840 \quad-0.1781760$

$\mathrm{H} \quad-3.8959510 \quad 1.0922710 \quad-0.5359340$

$\begin{array}{llll}\mathrm{H} & -2.8671710 & 1.6896750 & 0.7630050\end{array}$

$\begin{array}{llll}\mathrm{C} & -1.9880190 & 1.8431050 & -1.2248450\end{array}$

$\mathrm{H} \quad-2.5594120 \quad 2.6942820 \quad-1.6175950$

$\begin{array}{llll}\mathrm{H} & -1.7989800 & 1.1654460 & -2.0639100\end{array}$

$\begin{array}{llll}\text { C } & 2.5760540 & -1.4161120 & 0.1480480\end{array}$

$\mathrm{H} \quad 3.3686260 \quad-1.1986550 \quad 0.8689510$

$\mathrm{H} \quad 2.8474140 \quad-0.9574510 \quad-0.8096580$ 


$\begin{array}{lrrr}\mathrm{H} & 2.5333710 & -2.4998660 & -0.0058340 \\ \mathrm{C} & -0.7027580 & 2.3514450 & -0.6513800 \\ \mathrm{H} & -0.7816880 & 3.0678170 & 0.1674730 \\ \mathrm{C} & 0.5217490 & 1.9633360 & -1.0093120 \\ \mathrm{H} & 0.6903160 & 1.2323380 & -1.7942060 \\ \mathrm{C} & 1.6933670 & 2.3957180 & -0.2238190 \\ \mathrm{O} & 2.8185850 & 1.8177560 & -0.6726180 \\ \mathrm{C} & 3.9653150 & 1.9970810 & 0.1552610 \\ \mathrm{H} & 4.7783080 & 1.4795670 & -0.3537040 \\ \mathrm{H} & 3.7845990 & 1.5505120 & 1.1369670 \\ \mathrm{H} & 4.1985950 & 3.0579600 & 0.2732890 \\ \mathrm{O} & 1.6667800 & 3.1411140 & 0.7364470 \\ \mathrm{C} & -0.4553310 & 0.2050480 & 1.8210420 \\ \mathrm{H} & -0.8720610 & -0.1769580 & 2.7637920 \\ \mathrm{H} & -0.6212550 & 1.2904030 & 1.8122770\end{array}$

\section{Calculated endo product for entry 3:}

Single point: SCF $(\omega \mathrm{B} 97 \mathrm{xd} / 6-311 \mathrm{G}(\mathrm{d}, \mathrm{p}))=-1289.829406$

Sum of electronic and thermal Free Energies $(\omega \mathrm{B} 97 \mathrm{xd} / 6-31 \mathrm{G}(\mathrm{d}))=-1289.208133$

$\mathrm{SCF}(\omega \mathrm{B} 97 \mathrm{xd} / 6-31 \mathrm{G}(\mathrm{d}))=-1289.545168$

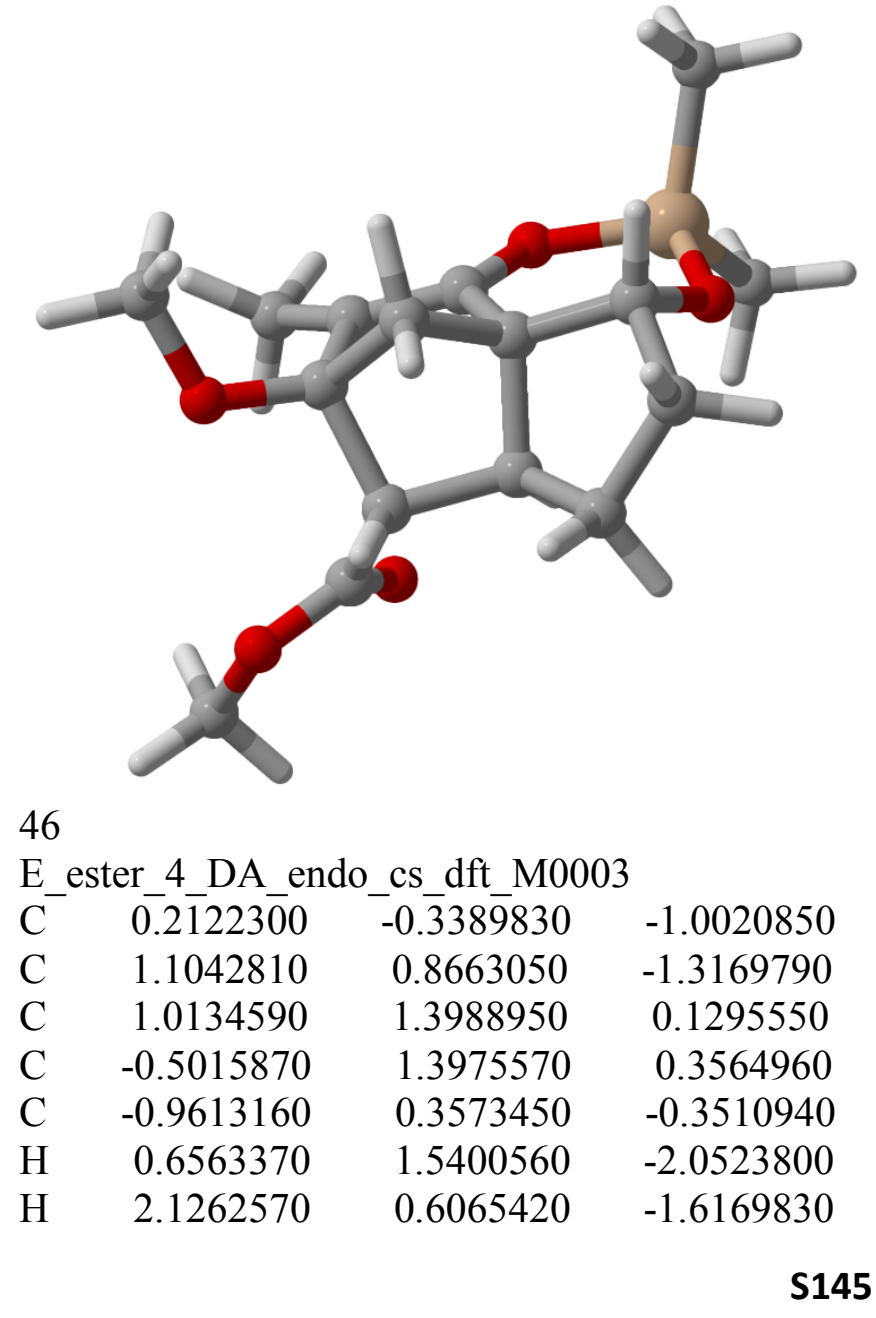




\begin{tabular}{lccc}
$\mathrm{C}$ & 1.0238300 & -1.0299520 & 0.1442620 \\
$\mathrm{C}$ & 1.6621170 & 0.1653290 & 0.8815610 \\
$\mathrm{C}$ & -0.0064500 & -1.4281570 & -2.0350400 \\
$\mathrm{H}$ & -0.2176590 & -1.0346780 & -3.0391230 \\
$\mathrm{C}$ & 1.2975070 & -2.2391730 & -2.0010560 \\
$\mathrm{H}$ & 1.9696510 & -1.8767140 & -2.7859360 \\
$\mathrm{H}$ & 1.0781620 & -3.2875530 & -2.2174800 \\
$\mathrm{C}$ & 1.9341670 & -2.0298630 & -0.5961570 \\
$\mathrm{H}$ & 2.9520780 & -1.6377460 & -0.6956050 \\
$\mathrm{O}$ & -2.2082130 & -0.1678240 & -0.3497200 \\
$\mathrm{O}$ & -1.0912800 & -2.2584290 & -1.6158540 \\
$\mathrm{Si}$ & -2.5522850 & -1.5680740 & -1.2264300 \\
$\mathrm{C}$ & -3.4935110 & -1.0634890 & -2.7574430 \\
$\mathrm{H}$ & -2.8942980 & -0.3911860 & -3.3814190 \\
$\mathrm{H}$ & -4.4161550 & -0.5353210 & -2.4935780 \\
$\mathrm{H}$ & -3.7618100 & -1.9364560 & -3.3622720 \\
$\mathrm{C}$ & -3.4842920 & -2.7428820 & -0.1325400 \\
$\mathrm{H}$ & -2.8940460 & -2.9797300 & 0.7578890 \\
$\mathrm{H}$ & -3.7047140 & -3.6784050 & -0.6574630 \\
$\mathrm{H}$ & -4.4330750 & -2.3044990 & 0.1943780 \\
$\mathrm{C}$ & -1.2134180 & 2.2220660 & 1.3769890 \\
$\mathrm{H}$ & -0.8860570 & 1.9547630 & 2.3884960 \\
$\mathrm{H}$ & -2.2906850 & 2.0445470 & 1.3182210 \\
$\mathrm{H}$ & -1.0303560 & 3.2945090 & 1.2463030 \\
$\mathrm{O}$ & 1.6928090 & 2.5677950 & 0.4747600 \\
$\mathrm{C}$ & 1.4339110 & 3.6643840 & -0.3701830 \\
$\mathrm{H}$ & 1.9070220 & 3.5367810 & -1.3540450 \\
$\mathrm{H}$ & 1.8615490 & 4.5436840 & 0.1159790 \\
$\mathrm{H}$ & 0.3574810 & 3.8232400 & -0.5208570 \\
$\mathrm{H}$ & 2.7425700 & 0.2297050 & 0.7246060 \\
$\mathrm{C}$ & 1.3963330 & 0.1882560 & 2.3655170 \\
$\mathrm{O}$ & 0.4312590 & -0.2949730 & 2.9145500 \\
$\mathrm{O}$ & 2.3620830 & 0.8458710 & 3.0241390 \\
$\mathrm{C}$ & 2.1500410 & 0.9937340 & 4.4252410 \\
$\mathrm{H}$ & 2.0687250 & 0.0174330 & 4.9098790 \\
$\mathrm{H}$ & 1.2350010 & 1.5605400 & 4.6168680 \\
& 3.0181740 & 1.5361220 & 4.7989610 \\
$\mathrm{H}$ & 0.3426920 & -1.5595080 & 0.8143230 \\
\hline & 2.0114840 & -2.9718620 & -0.0472510
\end{tabular}

\section{Calculated exo product for entry 3:}

Single point: SCF $(\omega \mathrm{B} 97 \mathrm{xd} / 6-311 \mathrm{G}(\mathrm{d}, \mathrm{p}))=-1289.820803$

Sum of electronic and thermal Free Energies $(\omega \mathrm{B} 97 \mathrm{xd} / 6-31 \mathrm{G}(\mathrm{d}))=-1289.198197$ $\operatorname{SCF}(\omega \mathrm{B} 97 \mathrm{xd} / 6-31 \mathrm{G}(\mathrm{d}))=-1289.536825$ 


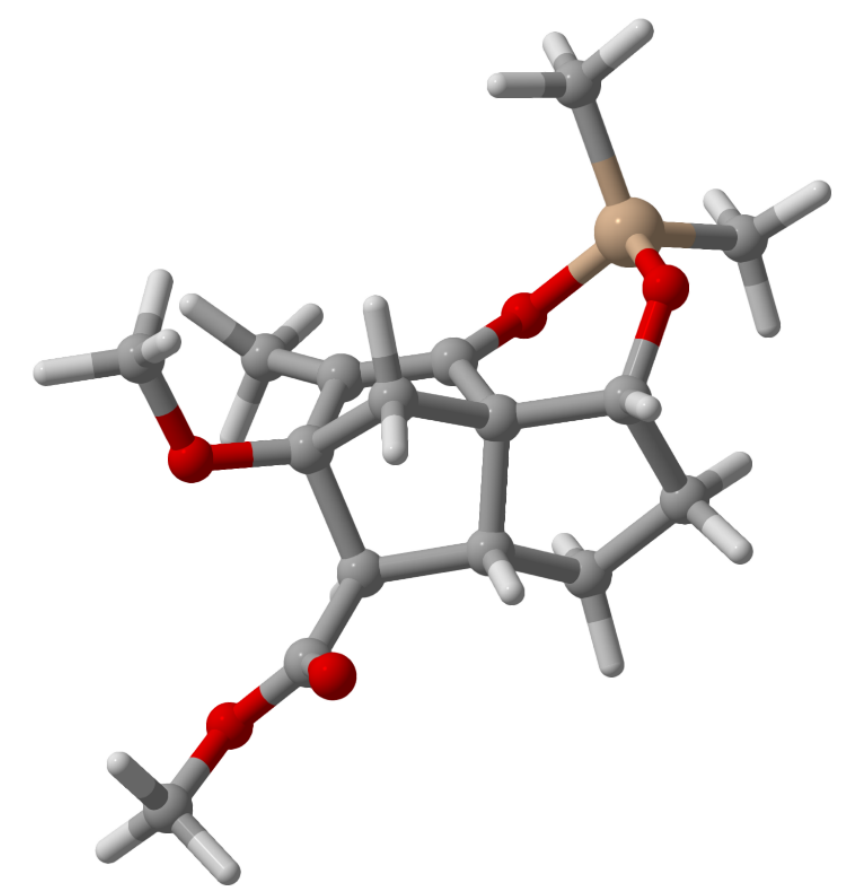

46

E_ester_4_DA_exo_cs_dft_M0001

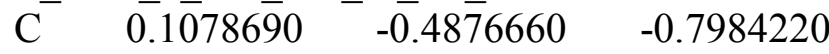

$\begin{array}{llll}\text { C } & 0.5773320 & 0.9062890 & -1.2290120\end{array}$

$\begin{array}{llll}\text { C } & 0.6416030 & 1.3992650 & 0.2472820\end{array}$

$\begin{array}{llll}\text { C } & -0.7517650 & 1.0392170 & 0.7500160\end{array}$

$\begin{array}{llll}\text { C } & -1.0471460 & -0.1024350 & 0.1223970\end{array}$

$\mathrm{H} \quad-0.1621090 \quad 1.4585210 \quad-1.8146510$

$\mathrm{H} \quad 1.5527020 \quad 0.9124190 \quad-1.7248560$

$\begin{array}{llll}\text { C } & 1.3165060 & -0.9071210 & 0.0848610\end{array}$

$\begin{array}{llll}\text { C } & 1.6068780 & 0.3426180 & 0.9318520\end{array}$

C $\quad-0.1562270 \quad-1.7422890 \quad-1.6301390$

$\mathrm{H} \quad 0.4586070 \quad-1.7343300 \quad-2.5346880$

C $\quad 0.3017220 \quad-2.9113570 \quad-0.6845540$

$\mathrm{H} \quad 1.0646290 \quad-3.5104250 \quad-1.1914630$

$\mathrm{H} \quad-0.5364030 \quad-3.5816480 \quad-0.4761000$

C $\quad 0.8903390 \quad-2.2743960 \quad 0.6030560$

$\mathrm{H} \quad 1.7107610 \quad-2.8656870 \quad 1.0196670$

$\mathrm{H} \quad 0.1204910 \quad-2.1722180 \quad 1.3761300$

O $\quad-2.1109740 \quad-0.9229820 \quad 0.3343420$

$\begin{array}{llll}\mathrm{O} & -1.5005590 & -1.8599640 & -2.0754240\end{array}$

$\begin{array}{llll}\mathrm{Si} & -2.7804050 & -1.6313600 & -1.0435410\end{array}$

$\begin{array}{llll}\text { C } & -3.9807560 & -0.4631000 & -1.8571600\end{array}$

$\mathrm{H} \quad-3.5025130 \quad 0.5074190 \quad-2.0265290$

$\mathrm{H} \quad-4.8667740 \quad-0.3036140 \quad-1.2334100$

$\mathrm{H} \quad-4.3113910 \quad-0.8487720 \quad-2.8275580$

C $\quad-3.5836830 \quad-3.2168400 \quad-0.4875300$

$\mathrm{H} \quad-2.8766740 \quad-3.8532490 \quad 0.0535550$ 


$\begin{array}{lrrr}\mathrm{H} & -3.9649680 & -3.7828150 & -1.3442350 \\ \mathrm{H} & -4.4270330 & -3.0079080 & 0.1800210 \\ \mathrm{C} & -1.4295180 & 1.7003060 & 1.9014440 \\ \mathrm{H} & -0.7780980 & 1.6998330 & 2.7847620 \\ \mathrm{H} & -2.3540440 & 1.1747040 & 2.1548990 \\ \mathrm{H} & -1.6722470 & 2.7482790 & 1.6915460 \\ \mathrm{O} & 1.0855310 & 2.6939320 & 0.5235580 \\ \mathrm{C} & 0.4791110 & 3.7012690 & -0.2519280 \\ \mathrm{H} & 0.8101960 & 3.6589460 & -1.2981430 \\ \mathrm{H} & 0.7855610 & 4.6562670 & 0.1796050 \\ \mathrm{H} & -0.6173180 & 3.6316370 & -0.2287100 \\ \mathrm{C} & 3.0401210 & 0.8086620 & 0.8244860 \\ \mathrm{O} & 3.7121440 & 0.7721080 & -0.1826720 \\ \mathrm{O} & 3.4840590 & 1.3041700 & 1.9867790 \\ \mathrm{C} & 4.7954450 & 1.8616380 & 1.9476860 \\ \mathrm{H} & 4.8353460 & 2.6899530 & 1.2360600 \\ \mathrm{H} & 5.5283650 & 1.1056400 & 1.6546500 \\ \mathrm{H} & 4.9950760 & 2.2164180 & 2.9585520 \\ \mathrm{H} & 2.1679760 & -1.0493370 & -0.5927780 \\ \mathrm{H} & 1.3422370 & 0.2400040 & 1.9862990\end{array}$

\section{Calculated endo transition state for entry 3:}

Single point: SCF $(\omega \mathrm{B} 97 \mathrm{xd} / 6-311 \mathrm{G}(\mathrm{d}, \mathrm{p}))=-1289.780979$

Sum of electronic and thermal Free Energies $(\omega \mathrm{B} 97 \mathrm{xd} / 6-31 \mathrm{G}(\mathrm{d}))=-1289.161276$ SCF $(\omega B 97 x d / 6-31 G(d))=-1289.494208$

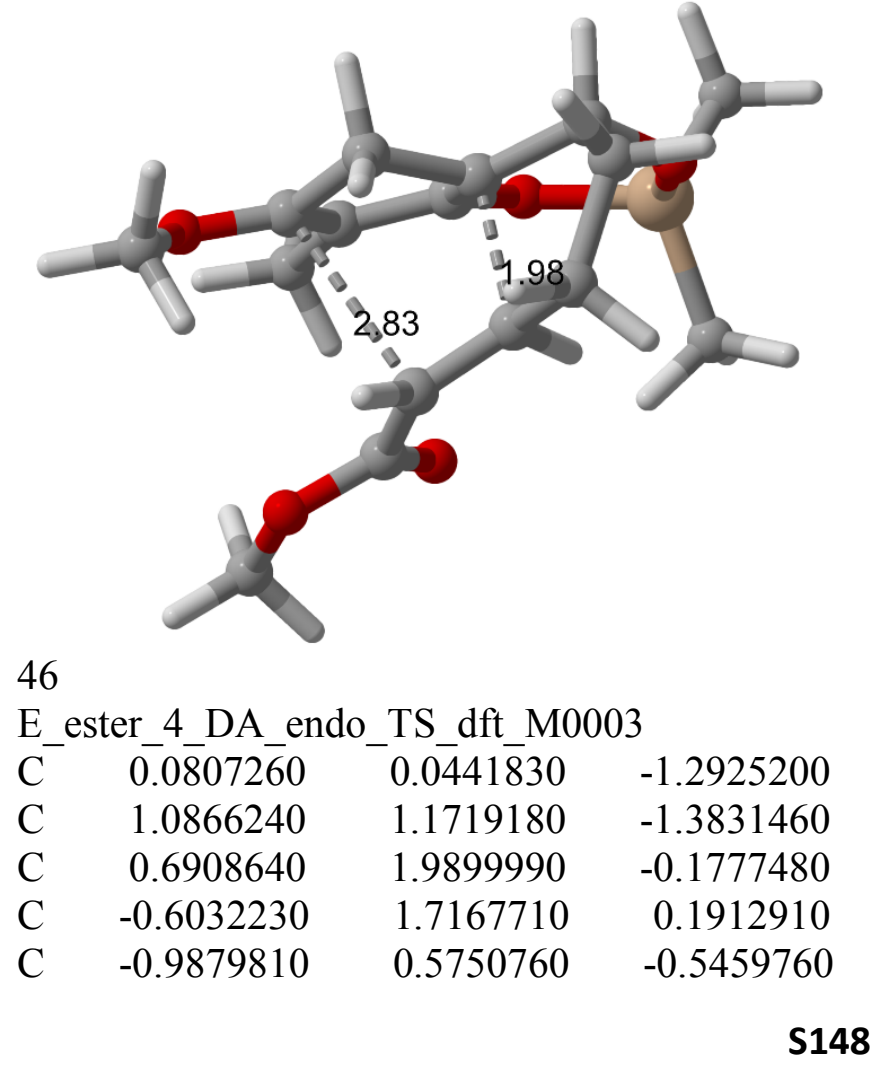




$\begin{array}{lrrr}\mathrm{H} & 0.9063650 & 1.7729550 & -2.2863860 \\ \mathrm{H} & 2.1287770 & 0.8486750 & -1.3884230 \\ \mathrm{C} & 1.0631540 & -1.1617230 & -0.0682200 \\ \mathrm{C} & 1.7113420 & -0.4161120 & 0.9189000 \\ \mathrm{C} & -0.1306500 & -0.9823530 & -2.3769120 \\ \mathrm{H} & -0.4387460 & -0.5268650 & -3.3291310 \\ \mathrm{C} & 1.1885680 & -1.7322780 & -2.5423230 \\ \mathrm{H} & 1.8387990 & -1.1462390 & -3.2027310 \\ \mathrm{H} & 1.0035710 & -2.6884830 & -3.0387510 \\ \mathrm{C} & 1.8439190 & -1.8975760 & -1.1499900 \\ \mathrm{H} & 2.8763870 & -1.5310810 & -1.1694550 \\ \mathrm{O} & -2.2007470 & 0.0242060 & -0.4456680 \\ \mathrm{O} & -1.1432170 & -1.9053010 & -1.9776510 \\ \mathrm{Si} & -2.4467380 & -1.5603080 & -1.0107070 \\ \mathrm{C} & -4.0301490 & -1.5390050 & -1.9880890 \\ \mathrm{H} & -3.9552240 & -0.8552620 & -2.8396100 \\ \mathrm{H} & -4.8687530 & -1.2139380 & -1.3627310 \\ \mathrm{H} & -4.2637450 & -2.5374620 & -2.3734160 \\ \mathrm{C} & -2.4789010 & -2.6967020 & 0.4602430 \\ \mathrm{H} & -1.7683900 & -2.3389530 & 1.2142840 \\ \mathrm{H} & -2.2116700 & -3.7212630 & 0.1806000 \\ \mathrm{H} & -3.4725340 & -2.7161350 & 0.9210650 \\ \mathrm{C} & -1.4100450 & 2.3676270 & 1.2645790 \\ \mathrm{H} & -0.8900400 & 3.2431700 & 1.6607180 \\ \mathrm{H} & -1.5729750 & 1.6506670 & 2.0765270 \\ \mathrm{H} & -2.3883610 & 2.6818850 & 0.8869450 \\ \mathrm{O} & 1.3711740 & 3.0280070 & 0.3161330 \\ \mathrm{C} & 2.7781690 & 3.0175790 & 0.1549340 \\ \mathrm{H} & 3.2063860 & 2.1344040 & 0.6402150 \\ \mathrm{H} & 3.1438640 & 3.9210410 & 0.6430740 \\ \mathrm{H} & 3.0609060 & 3.0384910 & -0.9041670 \\ \mathrm{H} & 2.7503460 & -0.1189100 & 0.8250250 \\ \mathrm{C} & 1.0033970 & -0.0947840 & 2.1247720 \\ \mathrm{O} & -0.1492750 & -0.4232400 & 2.3931000 \\ \mathrm{O} & 1.7465370 & 0.6626980 & 2.9797120 \\ \mathrm{H} & 1.0790300 & 1.0613360 & 4.1653220 \\ \mathrm{H} & 0.6961980 & 0.1970980 & 4.7150980 \\ \mathrm{H} & 1.2414160 & 1.7288080 & 3.9387070 \\ \mathrm{H} & 1.89598890 & -2.9549270 & -0.8717060\end{array}$

\section{Calculated exo transition state for entry 3:}

Single point: SCF $(\omega B 97 x d / 6-311 G(d, p))=-1289.772266$

Sum of electronic and thermal Free Energies $(\omega \mathrm{B} 97 \mathrm{xd} / 6-31 \mathrm{G}(\mathrm{d}))=-1289.15109$ 
$\operatorname{SCF}(\omega \mathrm{B} 97 \mathrm{xd} / 6-31 \mathrm{G}(\mathrm{d}))=-1289.4855$

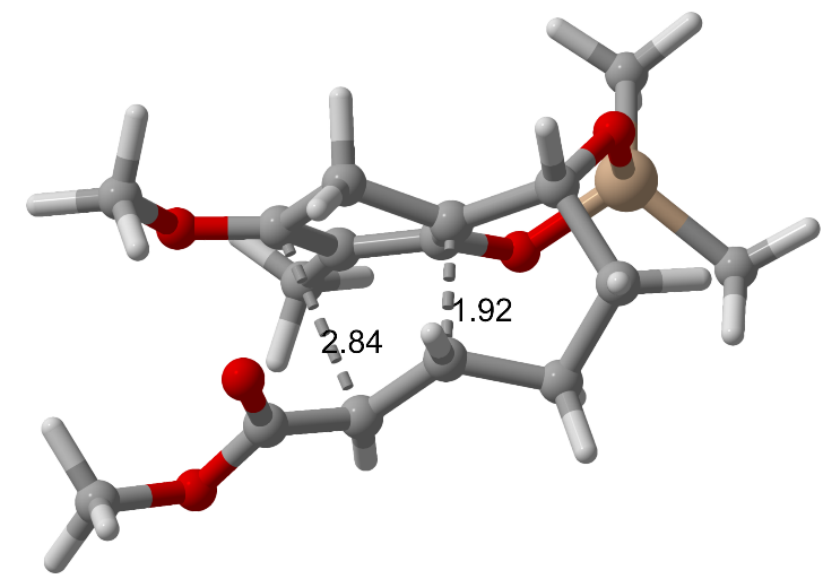

46

E_ester_4_DA_exo_TS_dft_M0001

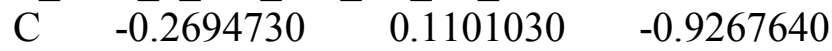

$\begin{array}{llll}\text { C } & 1.0527500 & -0.5627130 & -1.2601280\end{array}$

$\begin{array}{llll}\text { C } & 1.8509800 & -0.2339470 & -0.0308990\end{array}$

$\begin{array}{llll}\text { C } & 1.3149190 & 0.8515210 & 0.6355480\end{array}$

$\begin{array}{llll}\text { C } & 0.0856560 & 1.1122080 & 0.0152160\end{array}$

$\mathrm{H} \quad 1.5265630 \quad-0.0709610 \quad-2.1218640$

$\mathrm{H} \quad 0.9877300 \quad-1.6355600 \quad-1.4538070$

C $\quad-1.0559050 \quad-1.3253240 \quad 0.0838060$

$\begin{array}{llll}\text { C } & -0.2667280 & -1.6868410 & 1.1851860\end{array}$

C $\quad-1.4172880 \quad 0.4015150 \quad-1.8873670$

$\mathrm{H} \quad-1.1918060 \quad-0.0025850 \quad-2.8780710$

C $\quad-2.7048570 \quad-0.2604820 \quad-1.3200160$

$\mathrm{H} \quad-2.9357250 \quad-1.1535910 \quad-1.9119970$

$\mathrm{H} \quad-3.5541850 \quad 0.4195160 \quad-1.4300910$

C $\quad-2.4329290 \quad-0.6938300 \quad 0.1245940$

$\mathrm{H} \quad-3.1793350 \quad-1.4185590 \quad 0.4677860$

$\mathrm{H} \quad-2.4545170 \quad 0.1519860 \quad 0.8225260$

$\begin{array}{llll}\mathrm{O} & -0.7178270 & 2.1236530 & 0.3881040\end{array}$

$\begin{array}{llll}\mathrm{O} & -1.5982450 & 1.8090490 & -2.0880950\end{array}$

$\begin{array}{llll}\mathrm{Si} & -1.6645330 & 2.8627610 & -0.8162160\end{array}$

C $\quad-0.8772870 \quad 4.4624720 \quad-1.3427910$

$\mathrm{H} \quad 0.1527490 \quad 4.2913680 \quad-1.6707960$

$\mathrm{H} \quad-0.8609990 \quad 5.1839630 \quad-0.5190150$

$\mathrm{H} \quad-1.4263750 \quad 4.9130440 \quad-2.1766210$

$\begin{array}{llll}\text { C } & -3.3561220 & 3.1028830 & -0.0747960\end{array}$

$\mathrm{H} \quad-3.7775660 \quad 2.1566090 \quad 0.2780230$

$\mathrm{H} \quad-4.0478800 \quad 3.5328470 \quad-0.8072250$

$\mathrm{H} \quad-3.3044730 \quad 3.7863390 \quad 0.7802060$

C $\quad 1.8946140 \quad 1.5510230 \quad 1.8210860$

$\begin{array}{llll}\mathrm{H} & 1.7848620 & 0.9379440 & 2.7225900\end{array}$

$\mathrm{H} \quad 1.3815130 \quad 2.5009410 \quad 1.9907810$ 


$\begin{array}{lrrr}\mathrm{H} & 2.9627680 & 1.7450330 & 1.6829600 \\ \mathrm{O} & 3.0367180 & -0.7374040 & 0.2796330 \\ \mathrm{C} & 3.5435560 & -1.8033770 & -0.5249820 \\ \mathrm{H} & 2.8688020 & -2.6622480 & -0.4929130 \\ \mathrm{H} & 4.5092020 & -2.0612140 & -0.0902430 \\ \mathrm{H} & 3.6879680 & -1.4668940 & -1.5581770 \\ \mathrm{C} & 0.6061270 & -2.8178760 & 1.1006100 \\ \mathrm{O} & 0.8342380 & -3.5106610 & 0.1056720 \\ \mathrm{O} & 1.2229780 & -3.0775700 & 2.2877580 \\ \mathrm{C} & 2.1063350 & -4.1843530 & 2.2847870 \\ \mathrm{H} & 2.9756630 & -3.9999420 & 1.6442600 \\ \mathrm{H} & 1.6065720 & -5.0918330 & 1.9349150 \\ \mathrm{H} & 2.4327720 & -4.3067910 & 3.3188790 \\ \mathrm{H} & -1.0060210 & -2.0838410 & -0.7025260 \\ \mathrm{H} & -0.3259610 & -1.1583780 & 2.1304760\end{array}$

\section{Calculated silacycle diene for entry 4:}

Single point: SCF $(\omega \mathrm{B} 97 \mathrm{xd} / 6-311 \mathrm{G}(\mathrm{d}, \mathrm{p}))=-1880.856237$

Sum of electronic and thermal Free Energies $(\omega \mathrm{B} 97 \mathrm{xd} / 6-31 \mathrm{G}(\mathrm{d}))=-1880.1020$

$\operatorname{SCF}(\omega \mathrm{B} 97 \mathrm{xd} / 6-31 \mathrm{G}(\mathrm{d}))=-1880.504531$

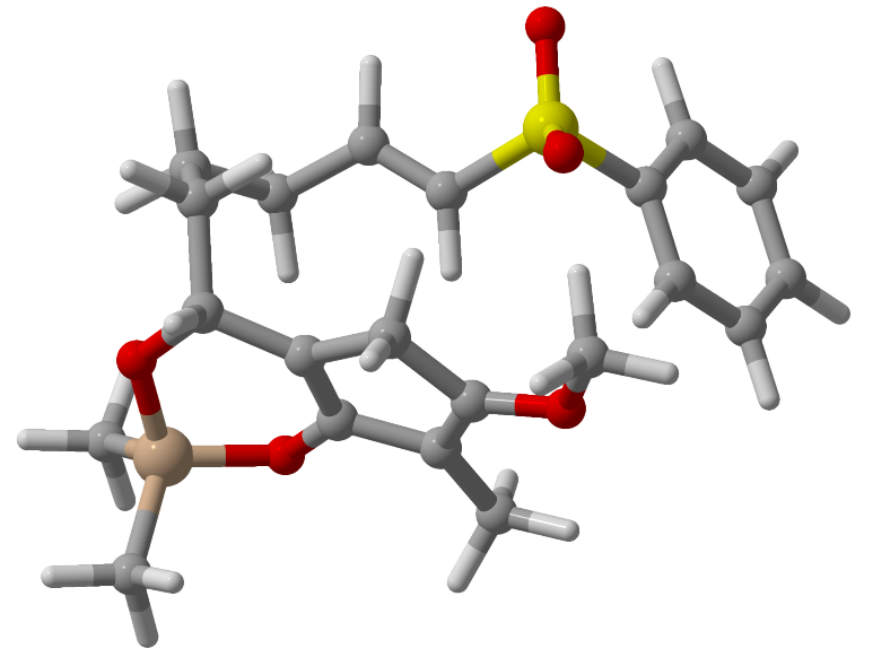

56

E $32 \_s m \_M 0001$

$\mathrm{O}-1.0668530 \quad-2.5217290 \quad 1.5931410$

C $\quad-0.0051940 \quad-1.8260610 \quad 1.1579760$

C $\quad-0.7344060 \quad-0.7702900 \quad 1.9553980$

C $\quad-1.8017560 \quad-0.3136290 \quad 1.0000320$

C $\quad-1.6729400 \quad-1.0367200 \quad-0.1324160$

C $\quad-0.5470680 \quad-1.9733810 \quad-0.0703200$

C $\quad-2.8491310 \quad 0.7225400 \quad 1.2964380$

O $\quad-3.8823880 \quad 0.7181870 \quad 0.3117730$

Si $\quad-4.0178920 \quad-0.3723990 \quad-0.9262730$ 


\begin{tabular}{|c|c|c|c|}
\hline $\mathrm{O}$ & -2.4572110 & -0.9470220 & -1.2412880 \\
\hline $\mathrm{C}$ & 1.4956770 & -2.2960490 & 2.9285420 \\
\hline $\mathrm{C}$ & -0.1199260 & -2.8817460 & -1.1764700 \\
\hline $\mathrm{C}$ & -2.3138500 & 2.1647570 & 1.4384940 \\
\hline $\mathrm{H}$ & -3.3177480 & 0.4654640 & 2.2588810 \\
\hline $\mathrm{C}$ & -2.1567920 & 2.9571430 & 0.1360990 \\
\hline $\mathrm{C}$ & -1.1301430 & 2.4271400 & -0.8802340 \\
\hline $\mathrm{C}$ & 0.2645890 & 2.4260040 & -0.3339130 \\
\hline $\mathrm{C}$ & 0.9607670 & 1.3185370 & -0.1013520 \\
\hline $\mathrm{C}$ & -4.6048050 & 0.5160420 & -2.4492780 \\
\hline $\mathrm{C}$ & -5.0809120 & -1.8246130 & -0.4379620 \\
\hline $\mathrm{H}$ & -1.1590900 & -1.1847090 & 2.8805640 \\
\hline $\mathrm{H}$ & -0.0433670 & 0.0324950 & 2.2477860 \\
\hline $\mathrm{H}$ & 0.7043100 & -2.5543030 & 3.6427030 \\
\hline $\mathrm{H}$ & 1.8114270 & -1.2585580 & 3.0663390 \\
\hline $\mathrm{H}$ & 2.3465850 & -2.9606470 & 3.0821680 \\
\hline $\mathrm{H}$ & 0.6566520 & -3.5679620 & -0.8282400 \\
\hline $\mathrm{H}$ & -0.9635400 & -3.4720710 & -1.5503040 \\
\hline $\mathrm{H}$ & 0.2795430 & -2.3158310 & -2.0266610 \\
\hline $\mathrm{H}$ & -3.0175450 & 2.7216330 & 2.0685880 \\
\hline $\mathrm{H}$ & -1.3642510 & 2.1287180 & 1.9868110 \\
\hline $\mathrm{H}$ & -1.8868490 & 3.9875560 & 0.4017300 \\
\hline $\mathrm{H}$ & -3.1314450 & 3.0066770 & -0.3591230 \\
\hline $\mathrm{H}$ & -1.4036340 & 1.4233530 & -1.2168480 \\
\hline $\mathrm{H}$ & -1.1580330 & 3.0824740 & -1.7608950 \\
\hline $\mathrm{H}$ & 0.7146560 & 3.3885480 & -0.0900190 \\
\hline $\mathrm{H}$ & 0.5954070 & 0.3134960 & -0.2925580 \\
\hline $\mathrm{H}$ & -3.9204440 & 1.3336280 & -2.6979530 \\
\hline $\mathrm{H}$ & -5.6023560 & 0.9417780 & -2.2983370 \\
\hline $\mathrm{H}$ & -4.6520970 & -0.1624180 & -3.3076920 \\
\hline $\mathrm{H}$ & -4.6308560 & -2.3511730 & 0.4106200 \\
\hline $\mathrm{H}$ & -5.1829960 & -2.5375910 & -1.2631850 \\
\hline $\mathrm{H}$ & -6.0837410 & -1.5005370 & -0.1398380 \\
\hline S & 2.5430140 & 1.3669140 & 0.6750780 \\
\hline $\mathrm{O}$ & 3.0579850 & 2.7341820 & 0.6540840 \\
\hline $\mathrm{O}$ & 2.4208480 & 0.6514060 & 1.9497530 \\
\hline $\mathrm{C}$ & 3.5333100 & 0.3612490 & -0.4152100 \\
\hline $\mathrm{C}$ & 4.9973340 & -1.1936290 & -2.1626360 \\
\hline $\mathrm{C}$ & 4.4268240 & 0.9786160 & -1.2835980 \\
\hline $\mathrm{C}$ & 3.3588980 & -1.0214810 & -0.3988740 \\
\hline $\mathrm{C}$ & 4.0966680 & -1.7967780 & -1.2865520 \\
\hline $\mathrm{C}$ & 5.1663000 & 0.1887910 & -2.1587210 \\
\hline $\mathrm{H}$ & 4.5387510 & 2.0574780 & -1.2600250 \\
\hline $\mathrm{H}$ & 2.6661910 & -1.4867100 & 0.2974150 \\
\hline $\mathrm{H}$ & 3.9706690 & -2.8749500 & -1.2888610 \\
\hline $\mathrm{H}$ & 5.8737800 & 0.6537890 & -2.8380470 \\
\hline
\end{tabular}


H $\quad 5.5729050 \quad-1.8055070 \quad-2.8508070$

\section{Calculated endo product for entry 4:}

Single point: SCF $(\omega \mathrm{B} 97 \mathrm{xd} / 6-311 \mathrm{G}(\mathrm{d}, \mathrm{p}))=-1880.894331$

Sum of electronic and thermal Free Energies $(\omega \mathrm{B} 97 \mathrm{xd} / 6-31 \mathrm{G}(\mathrm{d}))=-1880.1338$

$\operatorname{SCF}(\omega \mathrm{B} 97 \mathrm{xd} / 6-31 \mathrm{G}(\mathrm{d}))=-1880.545644$

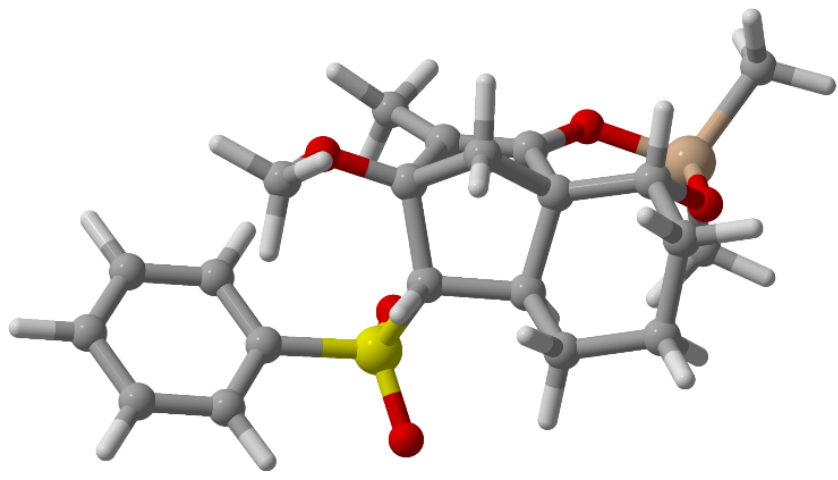

56

E_32_endo_M0002

$\mathrm{O}^{-}-2.54 \overline{3} 8050 \quad-1.7145980 \quad 0.1160570$

C $\quad-1.4833630 \quad-1.0215700 \quad 0.5905980$

$\begin{array}{llll}\text { C } & -0.2579590 & -1.4932080 & 0.8305210\end{array}$

C $\quad 0.5524810 \quad-0.2848190 \quad 1.2791420$

$\begin{array}{llll}\text { C } & -0.5291590 & 0.5279690 & 2.0398800\end{array}$

C $\quad-1.5217620 \quad 0.4775200 \quad 0.8654440$

C $\quad 0.2752740 \quad-2.8721330 \quad 0.6396340$

C $\quad 0.7573490 \quad 0.7078480 \quad 0.0714470$

C $\quad-0.6823220 \quad 1.1758580 \quad-0.2662300$

C $\quad-2.9277600 \quad 1.0504930 \quad 1.0359410$

$\begin{array}{llll}\mathrm{C} & -2.9387110 & 2.5748200 & 1.0716090\end{array}$

O $\quad-3.7351090 \quad 0.6518920 \quad-0.0723380$

$\mathrm{H} \quad-3.3646050 \quad 0.6539890 \quad 1.9654260$

$\mathrm{H} \quad-1.0052370 \quad 0.7982140 \quad-1.2422320$

$\begin{array}{llll}\text { O } & 1.6963440 & -0.6916300 & 1.9640390\end{array}$

$\begin{array}{llll}\mathrm{C} & 2.4900280 & 0.3176800 & 2.5407480\end{array}$

$\begin{array}{llll}\mathrm{Si} & -3.8928630 & -0.9383410 & -0.5206060\end{array}$

$\begin{array}{llll}\mathrm{C} & -3.8753370 & -1.0189730 & -2.3778900\end{array}$

$\begin{array}{llll}\text { C } & -5.3897240 & -1.7430490 & 0.2417970\end{array}$

C $\quad-2.2669770 \quad 3.1593890 \quad-0.1725970$

C $\quad-0.8108090 \quad 2.7040390 \quad-0.2625200$

$\mathrm{H} \quad-0.2232800 \quad 1.5450280 \quad 2.3131610$

$\mathrm{H} \quad-0.8769990 \quad-0.0086670 \quad 2.9261300$

$\mathrm{H} \quad 0.8145780 \quad-2.9551730 \quad-0.3100640$

$\mathrm{H} \quad-0.5497680 \quad-3.5896260 \quad 0.6187400$

$\mathrm{H} \quad 0.9562100 \quad-3.1411940 \quad 1.4533590$

$\mathrm{H} \quad 1.3571160 \quad 1.5662310 \quad 0.3932510$ 


$\begin{array}{lrrr}\mathrm{H} & -3.9780270 & 2.9102840 & 1.1485470 \\ \mathrm{H} & -2.4164930 & 2.9132320 & 1.9768260 \\ \mathrm{H} & 1.9047090 & 0.9711700 & 3.2016290 \\ \mathrm{H} & 2.9980550 & 0.9298710 & 1.7833720 \\ \mathrm{H} & 3.2512570 & -0.1933930 & 3.1327950 \\ \mathrm{H} & -4.6775840 & -0.4081540 & -2.8060740 \\ \mathrm{H} & -2.9210590 & -0.6481910 & -2.7662050 \\ \mathrm{H} & -4.0055990 & -2.0474620 & -2.7307970 \\ \mathrm{H} & -6.3141590 & -1.3026330 & -0.1470360 \\ \mathrm{H} & -5.3855200 & -1.6203360 & 1.3298920 \\ \mathrm{H} & -5.4087230 & -2.8167060 & 0.0251700 \\ \mathrm{H} & -2.8195990 & 2.8361850 & -1.0617610 \\ \mathrm{H} & -2.3130220 & 4.2534780 & -0.1444290 \\ \mathrm{H} & -0.3227940 & 3.0974530 & -1.1603010 \\ \mathrm{H} & -0.2639220 & 3.1157990 & 0.5981230 \\ \mathrm{~S} & 1.6359460 & 0.1562020 & -1.4047780 \\ \mathrm{O} & 1.1225760 & -1.1322250 & -1.8630670 \\ \mathrm{O} & 1.6393530 & 1.3053860 & -2.3128540 \\ \mathrm{C} & 3.3089970 & -0.0721150 & -0.8199480 \\ \mathrm{C} & 5.8604690 & -0.3599490 & 0.1953560 \\ \mathrm{C} & 4.1882310 & 1.0056430 & -0.8768750 \\ \mathrm{C} & 3.6869230 & -1.2948760 & -0.2751540 \\ \mathrm{C} & 4.9729460 & -1.4333600 & 0.2349910 \\ \mathrm{C} & 5.4727210 & 0.8557910 & -0.3633940 \\ \mathrm{H} & 3.8658400 & 1.9371490 & -1.3307260 \\ \mathrm{H} & 2.9824630 & -2.1177660 & -0.2469320 \\ \mathrm{H} & 5.2824620 & -2.3812310 & 0.6636780 \\ \mathrm{H} & 6.1714440 & 1.6854630 & -0.4049190 \\ \mathrm{H} & 6.8631110 & -0.4740330 & 0.5965900\end{array}$

\section{Calculated exo product for entry 4:}

Single point: SCF $(\omega \mathrm{B} 97 \mathrm{xd} / 6-311 \mathrm{G}(\mathrm{d}, \mathrm{p}))=-1880.887481$

Sum of electronic and thermal Free Energies $(\omega \mathrm{B} 97 \mathrm{xd} / 6-31 \mathrm{G}(\mathrm{d}))=-1880.1269$ $\operatorname{SCF}(\omega \mathrm{B} 97 \mathrm{xd} / 6-31 \mathrm{G}(\mathrm{d}))=-1880.539317$ 


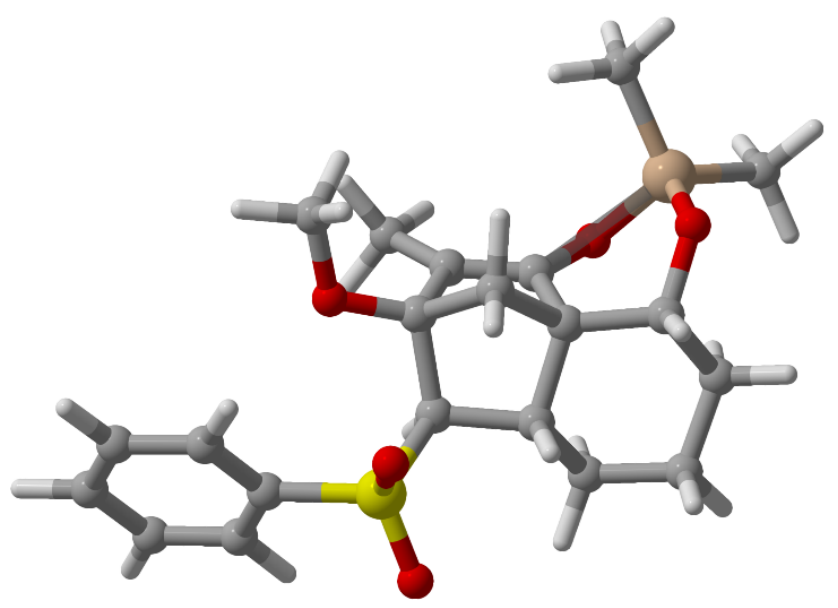

56

E 32 exo_M0001

$\begin{array}{llll}\mathrm{O} & -2.8854830 & -0.6545330 & 1.2414500\end{array}$

$\begin{array}{llll}\text { C } & 1.6967630 & -0.5865510 & 0.5811120\end{array}$

$\begin{array}{llll}\text { C } & 0.6066720 & -1.3419350 & 0.7344280\end{array}$

$\begin{array}{llll}\text { C } & -0.3788140 & -0.8238820 & -0.3170010\end{array}$

$\begin{array}{llll}\text { C } & 0.5892570 & -0.4265390 & -1.4641310\end{array}$

$\begin{array}{llll}\text { C } & 1.4548560 & 0.4385700 & -0.5265360\end{array}$

$\begin{array}{llll}\text { C } & 2.7397020 & 1.1256200 & -0.9858940\end{array}$

$\begin{array}{llll}\text { C } & 0.3684630 & 1.4676690 & -0.0815850\end{array}$

$\begin{array}{lrrr}\text { C } & -0.8476550 & 0.5628600 & 0.2213030\end{array}$

$\begin{array}{llll}\text { C } & 0.2733850 & -2.3632680 & 1.7671920\end{array}$

$\begin{array}{llll}\mathrm{O} & -1.4659140 & -1.6583920 & -0.5744350\end{array}$

$\begin{array}{llll}\text { C } & -1.1890570 & -2.7649960 & -1.3996660\end{array}$

$\begin{array}{llll}\mathrm{O} & 3.7857140 & 0.1630870 & -1.1276990\end{array}$

$\begin{array}{llll}\mathrm{H} & 2.5807670 & 1.5624080 & -1.9787810\end{array}$

$\begin{array}{llll}\mathrm{C} & 0.8465570 & 2.4425600 & 0.9778330\end{array}$

$\mathrm{H} \quad 0.1357890 \quad 2.0610350 \quad-0.9770050$

$\begin{array}{llll}\mathrm{Si} & 4.1855260 & -0.8134060 & 0.1613590\end{array}$

$\begin{array}{llll}\text { C } & 5.7193930 & -0.2775090 & 1.0698290\end{array}$

$\begin{array}{llll}\text { C } & 4.2856490 & -2.5686740 & -0.4532290\end{array}$

$\begin{array}{llll}\text { C } & 2.0449280 & 3.2010690 & 0.3958220\end{array}$

$\begin{array}{llll}\text { C } & 3.1673620 & 2.2377410 & -0.0057100\end{array}$

$\begin{array}{llll}\mathrm{H} & 0.1025620 & 0.1344660 & -2.2677160\end{array}$

$\mathrm{H} \quad 1.1325610 \quad-1.2848210 \quad-1.8666560$

$\begin{array}{llll}\mathrm{H} & -1.0912960 & 0.5172070 & 1.2872400\end{array}$

$\begin{array}{llll}\mathrm{H} & 0.0100800 & -3.3285180 & 1.3200510\end{array}$

$\mathrm{H} \quad 1.1199170 \quad-2.5129600 \quad 2.4425050$

$\begin{array}{llll}\mathrm{H} & -0.5927580 & -2.0474180 & 2.3623060\end{array}$

$\begin{array}{llll}\mathrm{H} & -0.9778590 & -2.4536430 & -2.4307150\end{array}$

$\mathrm{H} \quad-2.0857390 \quad-3.3881570 \quad-1.3904180$

$\begin{array}{llll}\mathrm{H} & -0.3392160 & -3.3534250 & -1.0265320\end{array}$

$\begin{array}{llll}\mathrm{H} & 1.1413040 & 1.9022070 & 1.8882570\end{array}$ 


$\begin{array}{lrrr}\mathrm{H} & 0.0378180 & 3.1312630 & 1.2408490 \\ \mathrm{H} & 5.6505980 & 0.7622360 & 1.4026480 \\ \mathrm{H} & 5.8831990 & -0.9076030 & 1.9510850 \\ \mathrm{H} & 6.5996150 & -0.3681780 & 0.4240810 \\ \mathrm{H} & 4.9728200 & -2.6525540 & -1.3018350 \\ \mathrm{H} & 4.6293580 & -3.2481850 & 0.3339090 \\ \mathrm{H} & 3.2970260 & -2.9030220 & -0.7856140 \\ \mathrm{H} & 1.7108440 & 3.7805680 & -0.4755620 \\ \mathrm{H} & 2.4349640 & 3.9233370 & 1.1208710 \\ \mathrm{H} & 3.5362840 & 1.7658670 & 0.9117540 \\ \mathrm{H} & 4.0104610 & 2.7788790 & -0.4486950 \\ \mathrm{~S} & -2.3206600 & 1.2809640 & -0.5583590 \\ \mathrm{O} & -2.4483340 & 2.6246470 & 0.0122260 \\ \mathrm{O} & -2.2308060 & 1.1079280 & -2.0089590 \\ \mathrm{C} & -3.7041570 & 0.3235730 & 0.0406310 \\ \mathrm{C} & -5.8553920 & -1.1313930 & 0.9757960 \\ \mathrm{C} & -4.2049670 & 0.5932440 & 1.3101910 \\ \mathrm{C} & -4.2677150 & -0.6508740 & -0.7751190 \\ \mathrm{C} & -5.3529650 & -1.3780300 & -0.3004760 \\ \mathrm{C} & -5.2849100 & -0.1477440 & 1.7797640 \\ \mathrm{H} & -3.7674280 & 1.3858320 & 1.9087670 \\ \mathrm{H} & -3.8541160 & -0.8240260 & -1.7617560 \\ \mathrm{H} & -5.8088880 & -2.1380570 & -0.9273940 \\ \mathrm{H} & -5.6871090 & 0.0510120 & 2.7681700 \\ \mathrm{H} & -6.7016350 & -1.7044590 & 1.3429130\end{array}$

\section{Calculated endo transition state for entry 4:}

Single point: SCF $(\omega \mathrm{B} 97 \mathrm{xd} / 6-311 \mathrm{G}(\mathrm{d}, \mathrm{p}))=-1880.827808$

Sum of electronic and thermal Free Energies $(\omega \mathrm{B} 97 \mathrm{xd} / 6-31 \mathrm{G}(\mathrm{d}))=-1880.0712$ $\operatorname{SCF}(\omega \mathrm{B} 97 \mathrm{xd} / 6-31 \mathrm{G}(\mathrm{d}))=-1880.476587$ 


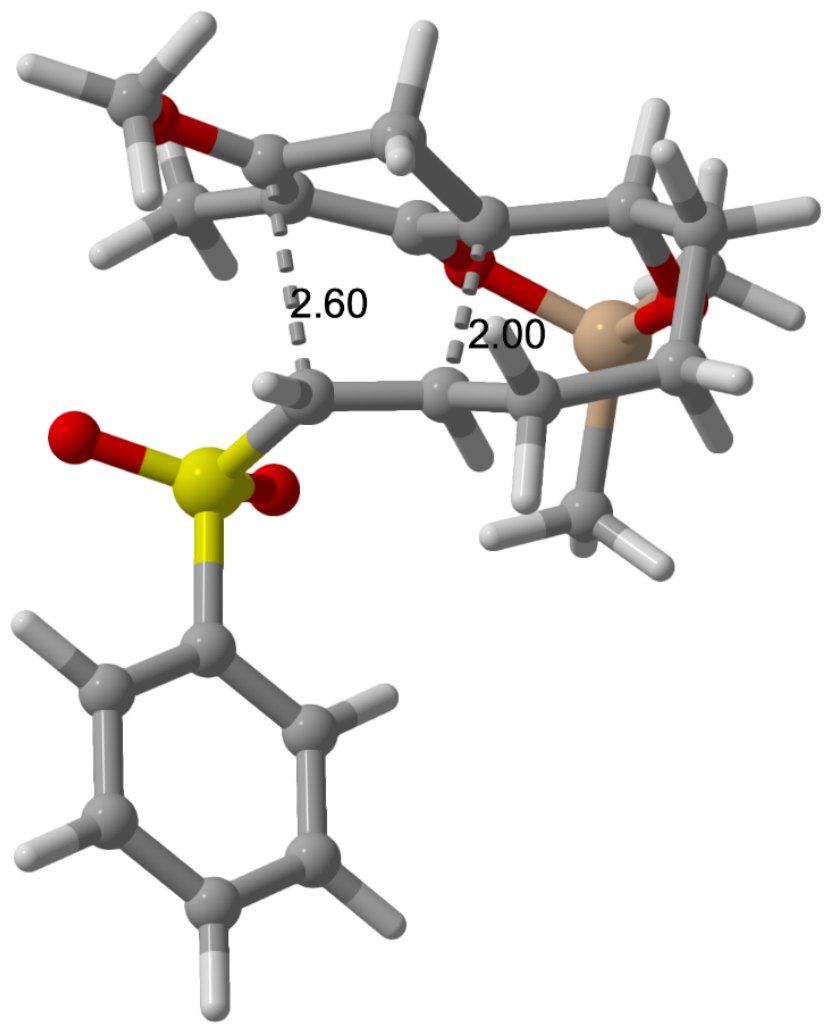

56

E_32_endo_TS_M0004

$\begin{array}{lrrr}\mathrm{O} & 2.3790280 & -0.2126520 & -1.5338650 \\ \mathrm{C} & 1.8476530 & 0.7088750 & -0.7251440 \\ \mathrm{C} & 1.1814830 & 1.8367680 & -1.1959540 \\ \mathrm{C} & 0.7887130 & 2.5397060 & -0.0636600 \\ \mathrm{C} & 1.5145690 & 2.0213840 & 1.1515660 \\ \mathrm{C} & 1.8152040 & 0.6050620 & 0.7022020 \\ \mathrm{C} & 0.8402270 & 2.1650300 & -2.6112030 \\ \mathrm{C} & -0.9415370 & 0.7599570 & 0.7101130 \\ \mathrm{C} & 0.0268930 & -0.1782770 & 1.1361630 \\ \mathrm{C} & 2.8729870 & -0.2651750 & 1.3576590 \\ \mathrm{C} & 2.6272050 & -0.5072300 & 2.8415190 \\ \mathrm{O} & 2.9109920 & -1.5372670 & 0.7099360 \\ \mathrm{H} & 3.8605820 & 0.2098940 & 1.2389200 \\ \mathrm{H} & 0.1138090 & -1.0810740 & 0.5341350 \\ \mathrm{O} & 0.1818160 & 3.7167940 & -0.1587660 \\ \mathrm{C} & -0.3517240 & 4.2792750 & 1.0250440 \\ \mathrm{Si} & 2.7739790 & -1.7475030 & -0.9305520 \\ \mathrm{C} & 1.3840510 & -2.9265870 & -1.3084980 \\ \mathrm{C} & 4.3997590 & -2.2157910 & -1.7044620 \\ \mathrm{C} & 1.3139810 & -1.2406730 & 3.1010460 \\ \mathrm{C} & 0.0894920 & -0.4581920 & 2.6304490 \\ \mathrm{H} & 0.9543250 & 2.0974210 & 2.0844300\end{array}$




$\begin{array}{lrrr}\mathrm{H} & 2.4456300 & 2.5958420 & 1.2623370 \\ \mathrm{H} & -0.2047530 & 2.4803290 & -2.6755230 \\ \mathrm{H} & 0.9740520 & 1.2844860 & -3.2420470 \\ \mathrm{H} & 1.4687400 & 2.9773700 & -2.9938110 \\ \mathrm{H} & -1.4980200 & 1.3565830 & 1.4264250 \\ \mathrm{H} & 3.4677630 & -1.0886200 & 3.2356660 \\ \mathrm{H} & 2.6328290 & 0.4613080 & 3.3606800 \\ \mathrm{H} & 0.4349140 & 4.4813200 & 1.7615970 \\ \mathrm{H} & -1.1032660 & 3.6099860 & 1.4556860 \\ \mathrm{H} & -0.8219560 & 5.2160590 & 0.7279230 \\ \mathrm{H} & 1.2807640 & -3.6843350 & -0.5243560 \\ \mathrm{H} & 0.4478450 & -2.3615950 & -1.3881200 \\ \mathrm{H} & 1.5435370 & -3.4400310 & -2.2625900 \\ \mathrm{H} & 4.7206920 & -3.2078180 & -1.3684820 \\ \mathrm{H} & 5.1806000 & -1.4966200 & -1.4378540 \\ \mathrm{H} & 4.3171960 & -2.2397940 & -2.7964670 \\ \mathrm{H} & 1.3470490 & -2.2115910 & 2.5937800 \\ \mathrm{H} & 1.2182110 & -1.4454480 & 4.1734270 \\ \mathrm{H} & -0.8184220 & -1.0201110 & 2.8859000 \\ \mathrm{H} & 0.0208280 & 0.4875780 & 3.1864840 \\ \mathrm{~S} & -1.7943530 & 0.4931410 & -0.7626960 \\ \mathrm{O} & -2.3698890 & 1.7537660 & -1.2416930 \\ \mathrm{O} & -0.9446520 & -0.3163790 & -1.6499500 \\ \mathrm{C} & -3.1894490 & -0.5564140 & -0.3338770 \\ \mathrm{C} & -5.3000310 & -2.1756010 & 0.4427640 \\ \mathrm{C} & -3.0003340 & -1.9334380 & -0.2421680 \\ \mathrm{C} & -4.4235630 & 0.0186050 & -0.0490400 \\ \mathrm{C} & -5.4810300 & -0.7986880 & 0.3402030 \\ \mathrm{C} & -4.0614320 & -2.7425180 & 0.1479020 \\ \mathrm{H} & -2.0352110 & -2.3611550 & -0.4942710 \\ \mathrm{H} & -4.5486860 & 1.0914490 & -0.1527520 \\ \mathrm{H} & -6.4499590 & -0.3593920 & 0.5578670 \\ \mathrm{H} & -3.9244440 & -3.8176270 & 0.2154940 \\ \mathrm{H} & -6.1277650 & -2.8102170 & 0.7453350\end{array}$

\section{Calculated exo transition state for entry 4:}

Single point: $\mathrm{SCF}(\omega \mathrm{B} 97 \mathrm{xd} / 6-311 \mathrm{G}(\mathrm{d}, \mathrm{p}))=$ Sum of electronic and thermal Free Energies $(\omega \mathrm{B} 97 \mathrm{xd} / 6-31 \mathrm{G}(\mathrm{d}))=$ $\operatorname{SCF}(\omega \mathrm{B} 97 \mathrm{xd} / 6-31 \mathrm{G}(\mathrm{d}))=$ 


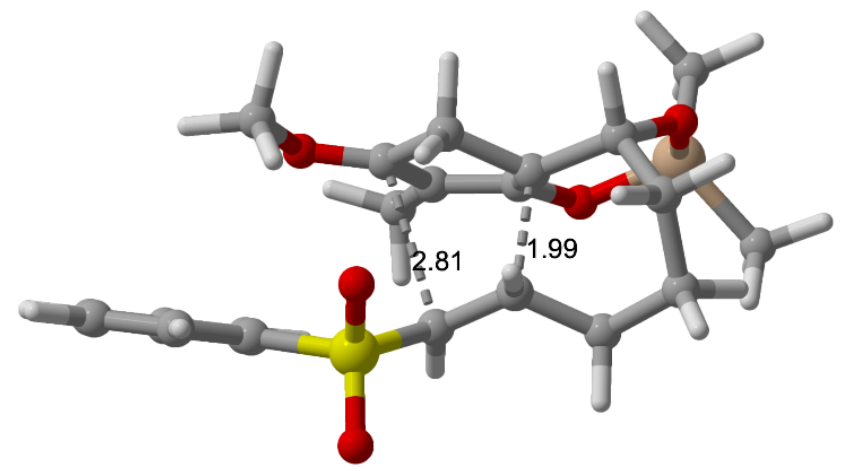

56

E_32_exo_TS_M0002

$\begin{array}{lccc}\mathrm{O} & -2.5441980 & -1.2191110 & -0.8535780 \\ \mathrm{C} & -1.5106780 & -0.9790600 & -0.0295340 \\ \mathrm{C} & -0.3342600 & -1.7405080 & -0.0938440 \\ \mathrm{C} & 0.4991400 & -1.2422380 & 0.8881010 \\ \mathrm{C} & -0.2169160 & -0.2339940 & 1.7374890 \\ \mathrm{C} & -1.3987360 & 0.1248580 & 0.8534610 \\ \mathrm{C} & -2.6556780 & 0.7662060 & 1.4520230 \\ \mathrm{C} & -0.4784040 & 1.5068490 & -0.2516690 \\ \mathrm{C} & 0.6854870 & 0.9766970 & -0.8279100 \\ \mathrm{C} & -0.0404510 & -2.8300230 & -1.0729390 \\ \mathrm{O} & 1.6779590 & -1.7769400 & 1.1587950 \\ \mathrm{C} & 2.4268280 & -1.2558030 & 2.2559010 \\ \mathrm{O} & -3.8627090 & 0.1576610 & 0.9902370 \\ \mathrm{H} & -2.6384920 & 0.5901310 & 2.5354450 \\ \mathrm{C} & -1.6481910 & 1.9721940 & -1.1000360 \\ \mathrm{H} & -0.2593390 & 2.1818060 & 0.5749680 \\ \mathrm{Si} & -4.1030240 & -0.8036410 & -0.3218130 \\ \mathrm{C} & -4.9652750 & 0.0435260 & -1.7363570 \\ \mathrm{C} & -4.9845570 & -2.3583750 & 0.1955770 \\ \mathrm{C} & -2.7362740 & 2.6770030 & -0.2581160 \\ \mathrm{C} & -2.7499190 & 2.2700800 & 1.2214980 \\ \mathrm{H} & 0.4123050 & 0.6108620 & 2.0270360 \\ \mathrm{H} & -0.5670160 & -0.7398050 & 2.6489520 \\ \mathrm{H} & 0.6714950 & 0.5939150 & -1.8447090 \\ \mathrm{H} & -0.8938570 & -3.5078920 & -1.1734370 \\ \mathrm{H} & 0.1622100 & -2.4191610 & -2.0695060 \\ \mathrm{H} & 0.8329950 & -3.4047610 & -0.7562810 \\ \mathrm{H} & 2.5394980 & -0.1723740 & 2.1738050 \\ \mathrm{H} & 3.4043060 & -1.7326340 & 2.1913820 \\ \mathrm{H} & 1.9407890 & -1.5203240 & 3.2018860 \\ \mathrm{H} & -2.0586660 & 1.1198830 & -1.6541670 \\ \mathrm{H} & -1.2683230 & 2.6666110 & -1.8572740 \\ \mathrm{H} & -4.4226860 & 0.9318900 & -2.0721340 \\ \mathrm{H} & -5.0548040 & -0.6393890 & -2.5888520\end{array}$




$\begin{array}{lrrr}\mathrm{H} & -5.9765050 & 0.3510990 & -1.4484910 \\ \mathrm{H} & -6.0111550 & -2.1443410 & 0.5121110 \\ \mathrm{H} & -5.0296090 & -3.0742000 & -0.6324940 \\ \mathrm{H} & -4.4661230 & -2.8353980 & 1.0333420 \\ \mathrm{H} & -2.5884000 & 3.7612680 & -0.3057170 \\ \mathrm{H} & -3.7218720 & 2.4876360 & -0.6951740 \\ \mathrm{H} & -3.6698600 & 2.6282510 & 1.6951330 \\ \mathrm{H} & -1.9203670 & 2.7475170 & 1.7565150 \\ \mathrm{~S} & 2.1983840 & 1.6542730 & -0.3547830 \\ \mathrm{O} & 2.6565640 & 2.6814600 & -1.2960660 \\ \mathrm{O} & 2.1188350 & 2.0297160 & 1.0725030 \\ \mathrm{C} & 3.4210560 & 0.3411250 & -0.4554800 \\ \mathrm{C} & 5.4277320 & -1.5741040 & -0.5671570 \\ \mathrm{C} & 3.2257080 & -0.8161850 & -1.1989390 \\ \mathrm{C} & 4.6164490 & 0.5530340 & 0.2295590 \\ \mathrm{C} & 5.6186550 & -0.4080780 & 0.1726880 \\ \mathrm{C} & 4.2331710 & -1.7754910 & -1.2515280 \\ \mathrm{H} & 2.2848020 & -0.9879420 & -1.7091110 \\ \mathrm{H} & 4.7468820 & 1.4594210 & 0.8123670 \\ \mathrm{H} & 6.5501130 & -0.2476570 & 0.7071850 \\ \mathrm{H} & 4.0781540 & -2.6850080 & -1.8239060 \\ \mathrm{H} & 6.2107980 & -2.3253670 & -0.6088160\end{array}$

Calculated silacycle diene for entry $\mathbf{5 / 6}$ :

Single point: SCF $(\omega B 97 x d / 6-311 G(d, p))=-1175.277267$

Sum of electronic and thermal Free Energies $(\omega \mathrm{B} 97 \mathrm{xd} / 6-31 \mathrm{G}(\mathrm{d}))=-1174.718394$ $\operatorname{SCF}(\omega \mathrm{B} 97 \mathrm{xd} / 6-31 \mathrm{G}(\mathrm{d}))=-1175.019593$

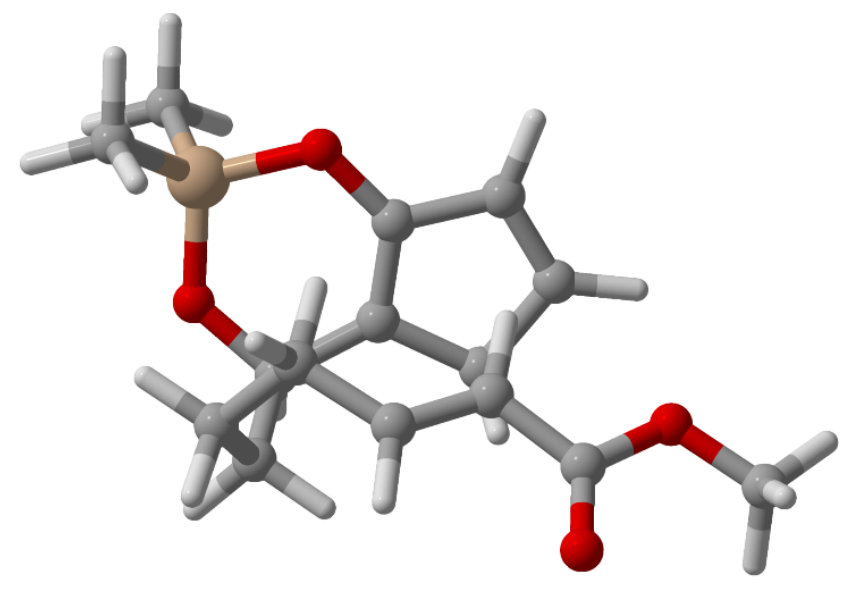

42

E_31_sm_M0053

$\mathrm{C}^{-}-0 . \overline{4355090} \quad 1.3360590 \quad-1.0169900$

$\begin{array}{llll}\text { C } & 0.4542900 & 2.5673960 & -0.2220810\end{array}$

C $\quad 1.2453920 \quad 2.3673220 \quad 0.8437290$ 


$\begin{array}{lrrr}\mathrm{C} & 1.8023200 & 0.9686390 & 0.8040370 \\ \mathrm{C} & 1.1960680 & 0.3831530 & -0.4398030 \\ \mathrm{O} & -0.2908610 & 1.2282440 & -2.1592620 \\ \mathrm{C} & 1.4597360 & -0.9929540 & -0.9795450 \\ \mathrm{C} & 0.8940420 & -2.1485740 & -0.1301330 \\ \mathrm{C} & -0.5978800 & -2.4501430 & -0.3027160 \\ \mathrm{C} & -1.5812460 & -1.3701560 & 0.1805230 \\ \mathrm{C} & -1.4386720 & -1.0856640 & 1.6421360 \\ \mathrm{C} & -1.1620940 & 0.1094800 & 2.1667820 \\ \mathrm{O} & 0.9962090 & -1.1288300 & -2.3206410 \\ \mathrm{Si} & 0.0962700 & -0.0509000 & -3.1942450 \\ \mathrm{C} & 1.0999250 & 0.6134820 & -4.6161520 \\ \mathrm{C} & -1.5063590 & -0.8243860 & -3.7413280 \\ \mathrm{C} & -0.9922480 & 0.2694310 & 3.6260960 \\ \mathrm{O} & -1.1574900 & -0.5896820 & 4.4646460 \\ \mathrm{O} & -0.5985670 & 1.5237090 & 3.9261010 \\ \mathrm{C} & -0.3974970 & 1.7789920 & 5.3128480 \\ \mathrm{H} & -0.0950280 & 3.4636150 & -0.4843430 \\ \mathrm{H} & 1.4578820 & 3.0727040 & 1.6369050 \\ \mathrm{H} & 2.9010210 & 0.9794230 & 0.7642030 \\ \mathrm{H} & 1.5249160 & 0.4127060 & 1.7101270 \\ \mathrm{H} & 2.5502560 & -1.1352830 & -1.0118320 \\ \mathrm{H} & 1.1268670 & -1.9474250 & 0.9234200 \\ \mathrm{H} & 1.4441070 & -3.0561220 & -0.4057040 \\ \mathrm{H} & -0.7922930 & -2.6569290 & -1.3603040 \\ \mathrm{H} & -0.8141630 & -3.3806030 & 0.2381710 \\ \mathrm{H} & -2.5996280 & -1.7397830 & -0.0055240 \\ \mathrm{H} & -1.4669300 & -0.4473490 & -0.3957510 \\ \mathrm{H} & -1.5504610 & -1.9284870 & 2.3255510 \\ \mathrm{H} & -1.0191460 & 0.9916460 & 1.5495010 \\ \mathrm{H} & 1.9962860 & 1.1208850 & -4.2445170 \\ \mathrm{H} & 0.5215230 & 1.3327160 & -5.2060670 \\ \mathrm{H} & 1.4214870 & -0.1935560 & -5.2833700 \\ \mathrm{H} & -2.1007040 & -1.1365790 & -2.8763940 \\ \mathrm{H} & -2.1042070 & -0.1142450 & -4.3230640 \\ \mathrm{H} & -1.3259280 & -1.7055430 & -4.3664730 \\ \mathrm{H} & -1.3211670 & 1.6149750 & 5.8738730 \\ & 0.3799390 & 1.1256260 & 5.7176890\end{array}$

\section{Calculated endo product for entry 5/6:}

Single point: SCF $(\omega \mathrm{B} 97 \mathrm{xd} / 6-311 \mathrm{G}(\mathrm{d}, \mathrm{p}))=-1175.316953$

Sum of electronic and thermal Free Energies $(\omega \mathrm{B} 97 \mathrm{xd} / 6-31 \mathrm{G}(\mathrm{d}))=-1174.756466$ $\operatorname{SCF}(\omega B 97 x d / 6-31 G(d))=-1175.064793$ 


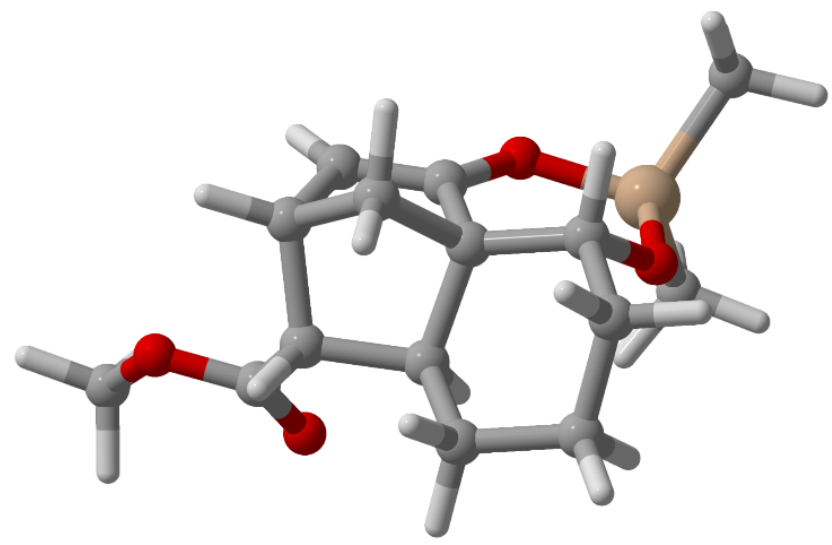

42

E_31_endo_M0002

$\begin{array}{llll}\mathrm{O}^{-} & -0.4690590 & 0.1696610 & -2.1925090\end{array}$

$\begin{array}{llll}\text { C } & 0.6565010 & 0.7518170 & -0.9901170\end{array}$

$\begin{array}{llll}\text { C } & 0.5422800 & 2.0476090 & -0.6816100\end{array}$

$\begin{array}{llll}\mathrm{C} & 0.7177810 & 2.1316600 & 0.8233260\end{array}$

$\begin{array}{llll}\mathrm{C} & 1.7516510 & 1.0148590 & 1.0567980\end{array}$

$\begin{array}{llll}\text { C } & 0.9343280 & -0.0420140 & 0.2887060\end{array}$

$\begin{array}{llll}\text { C } & 1.5249530 & -1.4312870 & 0.0672020\end{array}$

$\begin{array}{llll}\text { C } & -0.4075900 & -0.0247560 & 1.1056620\end{array}$

$\begin{array}{llll}\mathrm{C} & -0.5517940 & 1.4749490 & 1.4721070\end{array}$

$\begin{array}{llll}\mathrm{O} & 0.7317620 & -2.1394080 & -0.8894800\end{array}$

$\begin{array}{llll}\mathrm{H} & 2.5507740 & -1.3290940 & -0.3208320\end{array}$

$\begin{array}{llll}\text { C } & -0.3585540 & -0.9448040 & 2.3314200\end{array}$

$\begin{array}{lllr}\mathrm{H} & -1.2388260 & -0.3387310 & 0.4680060\end{array}$

$\begin{array}{llll}\mathrm{Si} & 0.4347510 & -1.5030890 & -2.3900280\end{array}$

$\begin{array}{llll}\text { C } & 1.7709460 & -1.9365780 & -3.6155480\end{array}$

$\begin{array}{llll}\text { C } & -1.2614460 & -2.0392540 & -2.9272330\end{array}$

$\begin{array}{llll}\text { C } & 0.1554170 & -2.3415110 & 1.9797420\end{array}$

$\begin{array}{llll}\text { C } & 1.5453200 & -2.2634660 & 1.3447190\end{array}$

$\begin{array}{llll}\mathrm{C} & -1.8428080 & 2.0980780 & 0.9946040\end{array}$

$\begin{array}{llll}\mathrm{O} & -2.7804100 & 1.5158250 & 0.5022100\end{array}$

$\begin{array}{llll}\mathrm{O} & -1.8329570 & 3.4282150 & 1.1985650\end{array}$

$\begin{array}{llll}\text { C } & -3.0043310 & 4.1185260 & 0.7699790\end{array}$

$\begin{array}{llll}\mathrm{H} & 0.2302130 & 2.8534120 & -1.3329590\end{array}$

$\begin{array}{llll}\mathrm{H} & 0.9531590 & 3.1176860 & 1.2241360\end{array}$

$\begin{array}{llll}\mathrm{H} & 2.7150710 & 1.2317760 & 0.5863140\end{array}$

$\begin{array}{llll}\mathrm{H} & 1.9059020 & 0.7690920 & 2.1136340\end{array}$

$\begin{array}{llll}\mathrm{H} & -0.5162290 & 1.6132580 & 2.5608250\end{array}$

$\begin{array}{llll}\mathrm{H} & -1.3593200 & -1.0008880 & 2.7760190\end{array}$

$\begin{array}{llll}\mathrm{H} & 0.2974240 & -0.5109320 & 3.0997280\end{array}$

$\begin{array}{llll}\mathrm{H} & 2.7567060 & -1.6493530 & -3.2343290\end{array}$

$\mathrm{H} \quad 1.7857210 \quad-3.0129400 \quad-3.8180680$

$\mathrm{H} \quad 1.6153480 \quad-1.4142760 \quad-4.5658020$ 


$\begin{array}{lrrr}\mathrm{H} & -1.5127400 & -1.6295540 & -3.9112660 \\ \mathrm{H} & -2.0128970 & -1.6909780 & -2.2114750 \\ \mathrm{H} & -1.3273110 & -3.1310200 & -2.9870700 \\ \mathrm{H} & -0.5307130 & -2.8267730 & 1.2761440 \\ \mathrm{H} & 0.1907590 & -2.9664280 & 2.8790600 \\ \mathrm{H} & 1.9198610 & -3.2628600 & 1.0998540 \\ \mathrm{H} & 2.2555040 & -1.8104090 & 2.0495230 \\ \mathrm{H} & -3.8854970 & 3.7516620 & 1.3025290 \\ \mathrm{H} & -2.8289250 & 5.1691220 & 1.0010020 \\ \mathrm{H} & -3.1588440 & 3.9831970 & -0.3034890\end{array}$

\section{Calculated exo product for entry 5/6:}

Single point: SCF $(\omega \mathrm{B} 97 \mathrm{xd} / 6-311 \mathrm{G}(\mathrm{d}, \mathrm{p}))=-1175.308703$

Sum of electronic and thermal Free Energies $(\omega \mathrm{B} 97 \mathrm{xd} / 6-31 \mathrm{G}(\mathrm{d}))=-1174.7562$

SCF $(\omega B 97 x d / 6-31 G(d))=-1175.064793$

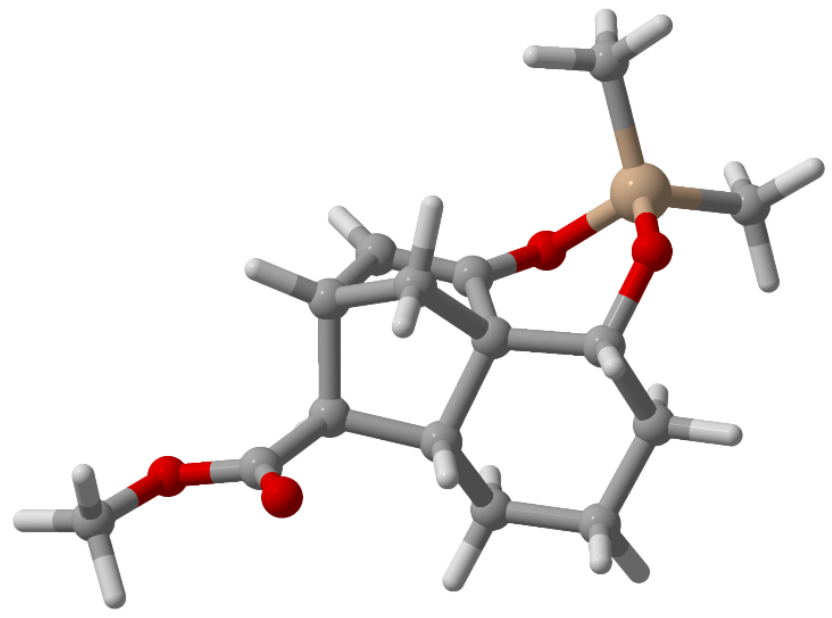

42

E_31_exo_M0002

$\mathrm{O}^{-} \quad 0.80 \overline{2} 2470 \quad-1.5613100 \quad-1.5855730$

C $\quad-0.1116970 \quad-0.6782160 \quad-1.1032310$

C $\quad-1.3979620 \quad-0.5275450 \quad-1.4271820$

C $\quad-1.8836820 \quad 0.6638410 \quad-0.6230500$

C $\quad-0.6057390 \quad 1.5422860 \quad-0.6089550$

C $\quad 0.2750410 \quad 0.3913840 \quad-0.0771740$

$\begin{array}{llll}\mathrm{C} & 1.7848230 & 0.5102240 & 0.1126770\end{array}$

C $\quad-1.9592750 \quad 0.1910700 \quad 0.8807820$

O $2.4485360 \quad 0.4869350 \quad-1.1531090$

$\mathrm{H} \quad 2.0158740 \quad 1.4806840 \quad 0.5677840$

C $\quad 0.0595330 \quad-1.0652020 \quad 2.0311510$

$\begin{array}{llll}\mathrm{Si} & 2.1855530 & -0.7875750 & -2.1917530\end{array}$

$\begin{array}{llll}\text { C } & 1.8395580 & -0.0929230 & -3.8844540\end{array}$

C $\quad 3.5526650 \quad-2.0521860 \quad-2.2019440$

C $\quad 1.5463910 \quad-0.8226240 \quad 2.3170320$ 


$\begin{array}{lrrr}\mathrm{C} & 2.3300110 & -0.6148900 & 1.0169440 \\ \mathrm{H} & -1.9985390 & -1.1462350 & -2.0813650 \\ \mathrm{H} & -2.8020590 & 1.1289090 & -0.9863220 \\ \mathrm{H} & -0.3239700 & 1.8777730 & -1.6103430 \\ \mathrm{H} & -0.6633010 & 2.3966330 & 0.0742470 \\ \mathrm{H} & -0.0710600 & -1.9797350 & 1.4360460 \\ \mathrm{H} & -0.4926780 & -1.2042880 & 2.9680870 \\ \mathrm{H} & 0.9046500 & 0.4771300 & -3.8695410 \\ \mathrm{H} & 2.6409080 & 0.5810070 & -4.2057170 \\ \mathrm{H} & 1.7423760 & -0.8891960 & -4.6300750 \\ \mathrm{H} & 3.2820750 & -2.9029120 & -2.8372230 \\ \mathrm{H} & 3.7631910 & -2.4308530 & -1.1975290 \\ \mathrm{H} & 4.4758230 & -1.6166520 & -2.5996620 \\ \mathrm{H} & 1.9800740 & -1.6654260 & 2.8664280 \\ \mathrm{H} & 1.6454680 & 0.0599630 & 2.9638110 \\ \mathrm{H} & 2.2899400 & -1.5563780 & 0.4578220 \\ \mathrm{H} & 3.3862310 & -0.4096000 & 1.2227280 \\ \mathrm{H} & -2.4479680 & -0.7846820 & 0.9344340 \\ \mathrm{C} & -0.4684980 & 0.1413090 & 1.2739790 \\ \mathrm{H} & -0.2721820 & 1.0248270 & 1.8952270 \\ \mathrm{C} & -2.7564340 & 1.1728910 & 1.7065480 \\ \mathrm{O} & -2.3242600 & 2.1654040 & 2.2490390 \\ \mathrm{O} & -4.0585400 & 0.8401080 & 1.7241910 \\ \mathrm{C} & -4.9117170 & 1.7496300 & 2.4165540 \\ \mathrm{H} & -4.8696940 & 2.7414460 & 1.9592330 \\ \mathrm{H} & -4.6163620 & 1.8318380 & 3.4655100 \\ \mathrm{H} & -5.9153530 & 1.3331350 & 2.3339470\end{array}$

Calculated endo transition state for entry 5/6:

Single point: SCF $(\omega \mathrm{B} 97 \mathrm{xd} / 6-311 \mathrm{G}(\mathrm{d}, \mathrm{p}))=-1175.248893$

Sum of electronic and thermal Free Energies $(\omega \mathrm{B} 97 \mathrm{xd} / 6-31 \mathrm{G}(\mathrm{d}))=-1174.6875$ $\operatorname{SCF}(\omega \mathrm{B} 97 \mathrm{xd} / 6-31 \mathrm{G}(\mathrm{d}))=-1174.994027$

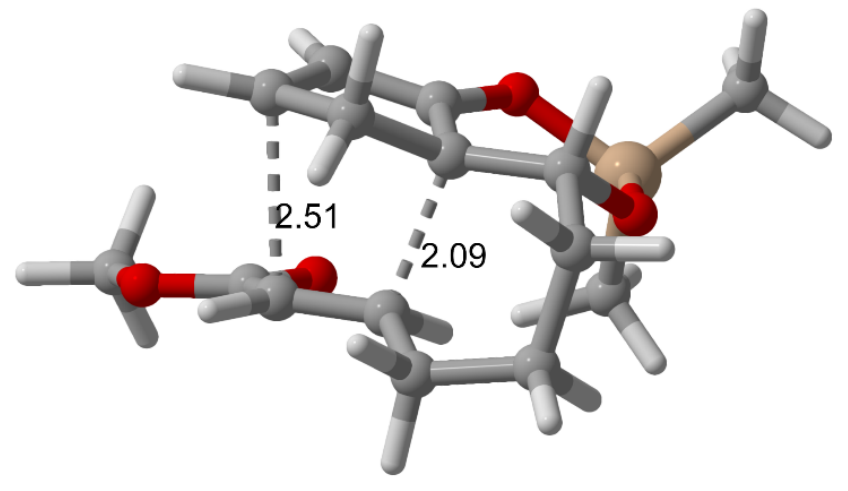

42

E_31_endo_TS_M0002 


\begin{tabular}{|c|c|c|c|}
\hline $\mathrm{O}$ & 0.7375020 & 0.3270790 & -2.0889400 \\
\hline $\mathrm{C}$ & 0.9315230 & 0.8144130 & -0.8538430 \\
\hline $\mathrm{C}$ & 0.8254040 & 2.1755590 & -0.5185150 \\
\hline $\mathrm{C}$ & 1.0831720 & 2.2916600 & 0.8258970 \\
\hline $\mathrm{C}$ & 1.7515380 & 1.0315730 & 1.2979100 \\
\hline $\mathrm{C}$ & 1.2177190 & 0.0358990 & 0.2890360 \\
\hline $\mathrm{C}$ & 1.7513130 & -1.3673790 & 0.0806040 \\
\hline $\mathrm{C}$ & -0.6256510 & -0.1638840 & 1.2488180 \\
\hline $\mathrm{C}$ & -1.0109020 & 1.1338210 & 1.5865110 \\
\hline $\mathrm{O}$ & 0.9563320 & -2.0415670 & -0.8953340 \\
\hline $\mathrm{H}$ & 2.7829340 & -1.3001810 & -0.3026490 \\
\hline $\mathrm{C}$ & -0.3251820 & -1.1546630 & 2.3577940 \\
\hline $\mathrm{H}$ & -1.1332120 & -0.5975220 & 0.3913080 \\
\hline $\mathrm{Si}$ & 0.3806820 & -1.3184190 & -2.2749280 \\
\hline $\mathrm{C}$ & 1.2954560 & -1.8961850 & -3.7891040 \\
\hline $\mathrm{C}$ & -1.4638010 & -1.5333300 & -2.3764360 \\
\hline $\mathrm{C}$ & 0.3704030 & -2.4431610 & 1.9201900 \\
\hline $\mathrm{C}$ & 1.7655780 & -2.2168120 & 1.3450240 \\
\hline $\mathrm{C}$ & -1.8357360 & 1.8765660 & 0.6585480 \\
\hline $\mathrm{O}$ & -2.1050940 & 1.5284760 & -0.4820800 \\
\hline $\mathrm{O}$ & -2.2728930 & 3.0521860 & 1.1721710 \\
\hline $\mathrm{C}$ & -3.0592880 & 3.8375610 & 0.2879360 \\
\hline $\mathrm{H}$ & 0.4606350 & 2.9438900 & -1.1872510 \\
\hline $\mathrm{H}$ & 1.0701790 & 3.2139130 & 1.3932190 \\
\hline $\mathrm{H}$ & 2.8382560 & 1.1434230 & 1.1605290 \\
\hline $\mathrm{H}$ & 1.5746140 & 0.7858480 & 2.3453990 \\
\hline $\mathrm{H}$ & -0.9024320 & 1.5149640 & 2.5962070 \\
\hline $\mathrm{H}$ & -1.2882830 & -1.4157940 & 2.8161100 \\
\hline $\mathrm{H}$ & 0.2569960 & -0.6669370 & 3.1513970 \\
\hline $\mathrm{H}$ & 2.3752980 & -1.7646260 & -3.6677220 \\
\hline $\mathrm{H}$ & 1.1009470 & -2.9571010 & -3.9805140 \\
\hline $\mathrm{H}$ & 0.9807910 & -1.3307770 & -4.6730620 \\
\hline $\mathrm{H}$ & -1.8227810 & -1.3913310 & -3.4015660 \\
\hline $\mathrm{H}$ & -1.9525520 & -0.7815980 & -1.7460020 \\
\hline $\mathrm{H}$ & -1.7694590 & -2.5305540 & -2.0422010 \\
\hline $\mathrm{H}$ & -0.2423460 & -2.9523670 & 1.1676330 \\
\hline $\mathrm{H}$ & 0.4416620 & -3.1183000 & 2.7807360 \\
\hline $\mathrm{H}$ & 2.2303350 & -3.1786690 & 1.1020320 \\
\hline $\mathrm{H}$ & 2.4096390 & -1.7252590 & 2.0871210 \\
\hline $\mathrm{H}$ & -3.9533380 & 3.2945850 & -0.0310360 \\
\hline $\mathrm{H}$ & -3.3388900 & 4.7288190 & 0.8511240 \\
\hline $\mathrm{H}$ & -2.4870660 & 4.1161830 & -0.6020690 \\
\hline
\end{tabular}

Calculated exo transition state for entry 5/6:

Single point: SCF $(\omega B 97 x d / 6-311 G(d, p))=-1175.234291$ 
Sum of electronic and thermal Free Energies $(\omega \mathrm{B} 97 \mathrm{xd} / 6-31 \mathrm{G}(\mathrm{d}))=-1174.6736$ $\operatorname{SCF}(\omega \mathrm{B} 97 \mathrm{xd} / 6-31 \mathrm{G}(\mathrm{d}))=-1174.979858$

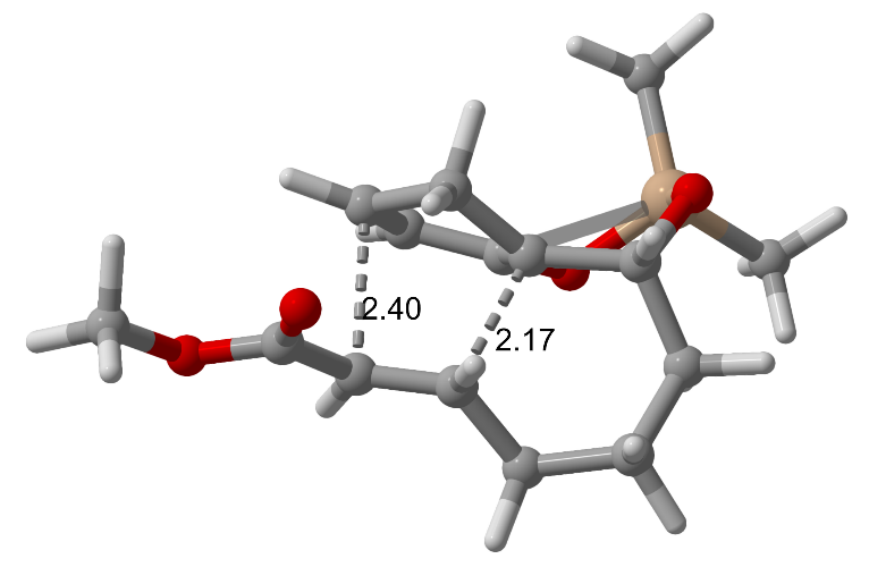

42

E_31_exo_TS_M0001

$\mathrm{O}-0.5 \overline{7} 07000 \quad-1.5012830 \quad-1.6479070$

$\begin{array}{llll}\text { C } & -0.2141730 & -0.5672140 & -1.0509150\end{array}$

$\begin{array}{llll}\text { C } & -1.6038370 & -0.4429070 & -1.1951920\end{array}$

$\begin{array}{llll}\text { C } & -1.9780050 & 0.7227310 & -0.5529990\end{array}$

$\begin{array}{llll}\mathrm{C} & -0.7455780 & 1.5505920 & -0.3151040\end{array}$

$\begin{array}{llll}\text { C } & 0.3022880 & 0.4598160 & -0.2419170\end{array}$

$\begin{array}{llll}\text { C } & 1.8071330 & 0.6162670 & -0.2280110\end{array}$

$\begin{array}{llll}\text { C } & -1.7161120 & -0.0680690 & 1.6928490\end{array}$

$\begin{array}{llll}\mathrm{O} & 2.3057560 & 0.5343080 & -1.5789860\end{array}$

$\begin{array}{llll}\mathrm{H} & 2.0764020 & 1.6114340 & 0.1408830\end{array}$

$\begin{array}{llll}\text { C } & 0.5956360 & -1.0896380 & 2.1351400\end{array}$

$\begin{array}{llll}\mathrm{Si} & 1.8504170 & -0.7705150 & -2.5091670\end{array}$

$\begin{array}{llll}\text { C } & 1.2163680 & -0.1347060 & -4.1407580\end{array}$

$\begin{array}{llll}\text { C } & 3.1738990 & -2.0648740 & -2.7066940\end{array}$

$\begin{array}{llll}\text { C } & 2.0598780 & -0.6517750 & 2.0422750\end{array}$

$\begin{array}{llll}\text { C } & 2.5346730 & -0.4550820 & 0.6053320\end{array}$

$\mathrm{H} \quad-2.2552170 \quad-1.1733880 \quad-1.6584220$

$\begin{array}{llll}\mathrm{H} & -2.9829120 & 1.1277020 & -0.5378330\end{array}$

$\mathrm{H} \quad-0.5648560 \quad 2.1450460 \quad-1.2245490$

$\begin{array}{llll}\mathrm{H} & -0.8109260 & 2.2326780 & 0.5328430\end{array}$

$\begin{array}{llll}\mathrm{H} & 0.4089220 & -1.9637760 & 1.4982680\end{array}$

$\begin{array}{lllr}\mathrm{H} & 0.3834920 & -1.3996900 & 3.1672160\end{array}$

$\begin{array}{llll}\mathrm{H} & 0.3243090 & 0.4804870 & -3.9816690\end{array}$

$\mathrm{H} \quad 1.9672440 \quad 0.4866260 \quad-4.6404390$

$\mathrm{H} \quad 0.9501220 \quad-0.9566920 \quad-4.8137240$

$\begin{array}{llll}\mathrm{H} & 2.7851010 & -2.9276440 & -3.2589100\end{array}$

$\mathrm{H} \quad 3.5444840 \quad-2.4179450 \quad-1.7400110$

$\mathrm{H} \quad 4.0242290 \quad-1.6624830 \quad-3.2679160$

$\begin{array}{llll}\mathrm{H} & 2.7000560 & -1.4078250 & 2.5109480\end{array}$

$\begin{array}{llll}\mathrm{H} & 2.1955720 & 0.2706120 & 2.6237110\end{array}$ 


$\begin{array}{lrrr}\mathrm{H} & 2.4218440 & -1.4178650 & 0.0949840 \\ \mathrm{H} & 3.6025360 & -0.2106400 & 0.5841090 \\ \mathrm{H} & -2.1919270 & -1.0325130 & 1.5537900 \\ \mathrm{C} & -0.3357860 & 0.0425250 & 1.7866410 \\ \mathrm{H} & 0.0029460 & 1.0053460 & 2.1712940 \\ \mathrm{C} & -2.5690490 & 1.0174040 & 2.1750210 \\ \mathrm{O} & -2.2118550 & 2.1461710 & 2.4636450 \\ \mathrm{O} & -3.8624510 & 0.6274540 & 2.2635590 \\ \mathrm{C} & -4.7624370 & 1.6270630 & 2.7255230 \\ \mathrm{H} & -4.7665530 & 2.4898950 & 2.0534280 \\ \mathrm{H} & -4.4871380 & 1.9674770 & 3.7273420 \\ \mathrm{H} & -5.7451970 & 1.1548920 & 2.7423210\end{array}$

Calculated silacycle diene for entry 7/8:

Single point: SCF $(\omega \mathrm{B} 97 \mathrm{xd} / 6-311 \mathrm{G}(\mathrm{d}, \mathrm{p}))=-1370.6541$

Sum of electronic and thermal Free Energies $(\omega \mathrm{B} 97 \mathrm{xd} / 6-31 \mathrm{G}(\mathrm{d}))=-1369.9343$

$\operatorname{SCF}(\omega \mathrm{B} 97 \mathrm{xd} / 6-31 \mathrm{G}(\mathrm{d}))=-1370.35425$

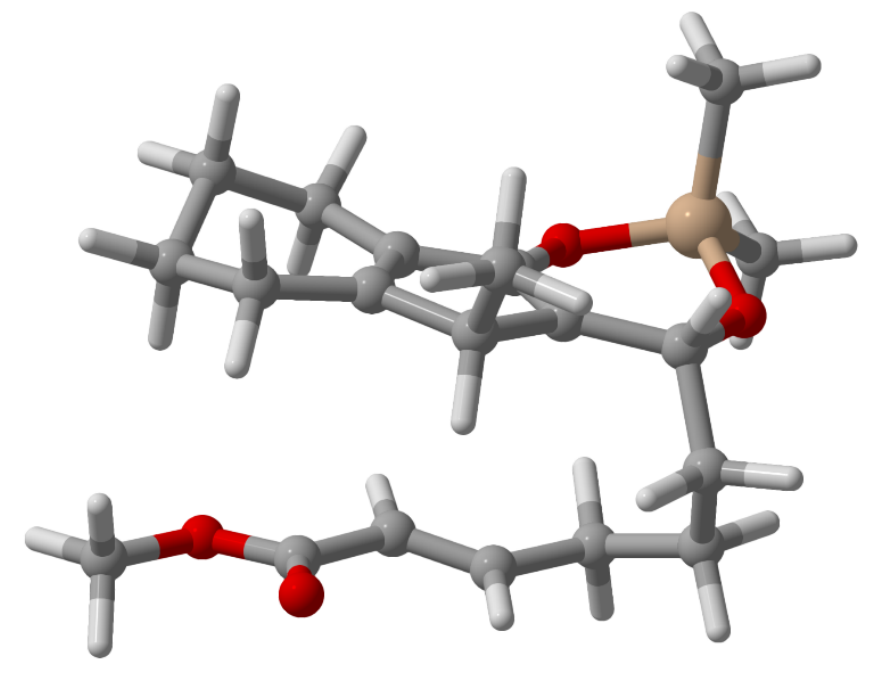

55

E_21_sm_alpha_M0006

$\begin{array}{lrrr}\mathrm{C}^{-} & -0.8170790 & -0.8352170 & -0.1393860 \\ \mathrm{C} & 0.4801790 & -1.5135100 & -0.1671860 \\ \mathrm{C} & 2.5872310 & -1.5371900 & 1.1993420 \\ \mathrm{C} & 2.1692360 & -3.2726580 & -0.6089320 \\ \mathrm{C} & 3.1819780 & -2.2974390 & 0.0035940 \\ \mathrm{C} & 0.9487310 & -2.5262830 & -1.1651040 \\ \mathrm{C} & 1.1966590 & -1.0795370 & 0.8851730 \\ \mathrm{C} & 0.3924400 & -0.0866650 & 1.6883580 \\ \mathrm{C} & -0.8990210 & 0.0079950 & 0.9100740 \\ \mathrm{O} & -1.7715930 & -1.0698340 & -1.0847850 \\ \mathrm{Si} & -3.3318890 & -0.6911050 & -0.5570370 \\ \mathrm{O} & -3.1808900 & 0.6549880 & 0.3968220\end{array}$




\begin{tabular}{|c|c|c|c|}
\hline $\mathrm{C}$ & -2.0791910 & 0.8606740 & 1.2829930 \\
\hline $\mathrm{C}$ & -1.7737500 & 2.3665640 & 1.3281970 \\
\hline $\mathrm{C}$ & -1.7514390 & 3.0888990 & -0.0240040 \\
\hline $\mathrm{C}$ & -0.7782340 & 2.5634650 & -1.0890500 \\
\hline $\mathrm{C}$ & 0.6387470 & 2.4500200 & -0.6125250 \\
\hline $\mathrm{C}$ & 1.5427160 & 1.5926000 & -1.0904090 \\
\hline $\mathrm{C}$ & 2.9008560 & 1.5483760 & -0.5173500 \\
\hline $\mathrm{O}$ & 3.2508990 & 2.0675820 & 0.5226230 \\
\hline $\mathrm{O}$ & 3.7395350 & 0.8379020 & -1.2972400 \\
\hline $\mathrm{C}$ & 5.0754170 & 0.7310680 & -0.8138700 \\
\hline $\mathrm{C}$ & -4.3544340 & -0.2529870 & -2.0443500 \\
\hline $\mathrm{C}$ & -3.9795450 & -2.1456570 & 0.4163820 \\
\hline $\mathrm{H}$ & 2.5763450 & -2.1842930 & 2.0898540 \\
\hline $\mathrm{H}$ & 3.2165200 & -0.6756250 & 1.4604670 \\
\hline $\mathrm{H}$ & 1.8372080 & -3.9817400 & 0.1612590 \\
\hline $\mathrm{H}$ & 2.6430200 & -3.8609780 & -1.4024370 \\
\hline $\mathrm{H}$ & 4.0881880 & -2.8311620 & 0.3114960 \\
\hline $\mathrm{H}$ & 3.4750490 & -1.5727790 & -0.76 \\
\hline $\mathrm{H}$ & 0.1388520 & -3.2247440 & -1.4065090 \\
\hline $\mathrm{H}$ & 1.2096210 & -2.0264800 & -2.1090900 \\
\hline $\mathrm{H}$ & -2.4088970 & 0.5725330 & 2.2945570 \\
\hline $\mathrm{H}$ & -2.5413490 & 2.8505340 & \\
\hline $\mathrm{H}$ & -0.8219380 & 2.5022150 & 1.8574800 \\
\hline $\mathrm{H}$ & -1.5177230 & 4.1436770 & 0.1723720 \\
\hline $\mathrm{H}$ & -2.7613770 & 3.0662400 & -0.4447820 \\
\hline $\mathrm{H}$ & -1.1112170 & 1.5953000 & -1.4773380 \\
\hline $\mathrm{H}$ & -0.8075320 & 3.2626600 & -1.9374360 \\
\hline $\mathrm{H}$ & 0.9584250 & 3.1229520 & 0.1841700 \\
\hline $\mathrm{H}$ & 1.3146270 & 0.8958790 & -1.8912930 \\
\hline $\mathrm{H}$ & 5.5215270 & 1.7209580 & -0.6906480 \\
\hline $\mathrm{H}$ & 5.0977830 & 0.2097080 & 0.1471490 \\
\hline $\mathrm{H}$ & 5.6156760 & 0.1575750 & -1.5668480 \\
\hline $\mathrm{H}$ & -3.9107680 & 0.5961930 & -2.5734080 \\
\hline $\mathrm{H}$ & -5.3740770 & 0.0213380 & -1.7543960 \\
\hline $\mathrm{H}$ & -4.4151360 & -1.0960160 & -2.7407410 \\
\hline $\mathrm{H}$ & -3.2895750 & -2.3868480 & 1.2325650 \\
\hline $\mathrm{H}$ & -4.9584910 & -1.9263350 & 0.8558570 \\
\hline $\mathrm{H}$ & -4.0790830 & -3.0354960 & -0.2144500 \\
\hline $\mathrm{H}$ & 0.9226460 & 0.8775920 & 1.6765190 \\
\hline $\mathrm{C}$ & 0.1980850 & -0.5208930 & 3.1474710 \\
\hline $\mathrm{H}$ & -0.3197700 & -1.4851110 & 3.1924400 \\
\hline $\mathrm{H}$ & -0.3957670 & 0.2145800 & 3.7012680 \\
\hline $\mathrm{H}$ & 1.1625430 & -0.6217140 & 3.6561900 \\
\hline
\end{tabular}

\section{Calculated endo product for entry 7/8:}


Single point: SCF $(\omega \mathrm{B} 97 \mathrm{xd} / 6-311 \mathrm{G}(\mathrm{d}, \mathrm{p}))=-1370.6862$

Sum of electronic and thermal Free Energies $(\omega \mathrm{B} 97 \mathrm{xd} / 6-31 \mathrm{G}(\mathrm{d}))=-1369.9617$

$\operatorname{SCF}(\omega \mathrm{B} 97 \mathrm{xd} / 6-31 \mathrm{G}(\mathrm{d}))=-1370.39122$

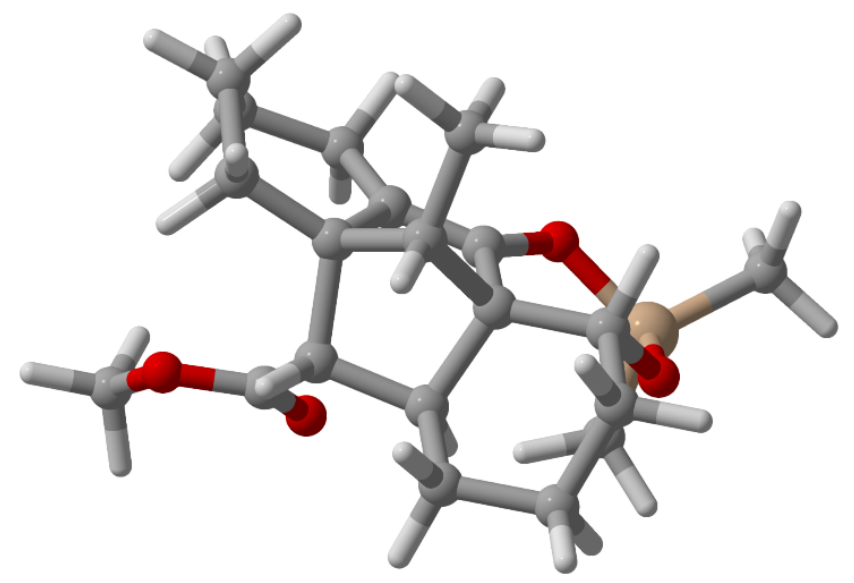

55

E_21_cis_M0002

O- -3.0384520

C $\quad-2.2726560$

0.1333380

$-0.1305960$

C $\quad-2.2283200$

1.0610290

0.6437100

C $\quad-0.2439840$

2.7048740

$-1.2687590$

C $\quad-0.7169790$

1.1516810

$-0.9666990$

C $\quad-0.7853090$

2.5343470

$-1.4338150$

C $\quad-2.6522210$

0.7788200

0.4564730

C $\quad-0.3905610$

2.4636020

0.1817470

C $\quad 0.9275940$

$-0.6712930$

0.7139700

$-0.7542910$

0.8981350

C $\quad 1.2880030$

1.0572550

$-0.7751560$

C $\quad 0.2656280$

1.4848180

1.3462400

C $\quad 1.9353060$

0.0379730

$-1.6797950$

O $\quad 1.3737690$

$-0.8695460$

$-2.2503610$

O $\quad 3.2670960$

0.2281400

$-1.7607200$

C $\quad 3.9757040$

$-0.7465720$

$-2.5233600$

$\mathrm{H} \quad-0.5779670$

0.4083450

$-1.6949900$

O $\quad-1.2881860$

$-1.6883250$

0.7062840

C $\quad 1.4519880$

0.6716520

0.7581310

C $\quad 1.7918250$

$-1.9578370$

1.1278360

C $\quad 2.8432580$

0.9233340

1.3534430

C $\quad 3.2518860$

$-1.5560580$

1.3967330

C $\quad 3.3286280$

$-0.2565720$

2.2039770

$-1.4969120$

$-0.1509640$

C $\quad-4.0287410$

$-2.4630500$

0.7603200

C $\quad-2.4873150$

$-2.0492050$

$-1.9142940$

$\mathrm{H} \quad-2.5371220$

0.9374480

1.7041770

$\mathrm{H} \quad-2.7561700$

1.9928990

$-1.9135940$

$\mathrm{H} \quad-2.5289030$

3.7102560

$-1.5842830$

$\mathrm{H} \quad-0.4261460$

2.6798640

$-2.4813680$ 


$\begin{array}{lrrr}\mathrm{H} & -0.2067320 & 3.3215870 & -0.8616350 \\ \mathrm{H} & -3.7342310 & 2.5877530 & 0.2956450 \\ \mathrm{H} & -2.1664620 & 3.1942550 & 0.8433370 \\ \mathrm{H} & 1.7755510 & 2.0238810 & -0.9468560 \\ \mathrm{H} & 5.0226070 & -0.4464370 & -2.4849060 \\ \mathrm{H} & 3.8465190 & -1.7413330 & -2.0883500 \\ \mathrm{H} & 3.6180350 & -0.7626520 & -3.5556450 \\ \mathrm{H} & 1.7325570 & -2.6457500 & 0.2747310 \\ \mathrm{H} & 3.5659370 & 1.0812460 & 0.5458340 \\ \mathrm{H} & 2.8319660 & 1.8466070 & 1.9466820 \\ \mathrm{H} & 3.7795360 & -1.4019140 & 0.4456930 \\ \mathrm{H} & 3.7701620 & -2.3696860 & 1.9153430 \\ \mathrm{H} & 4.3566500 & -0.0704670 & 2.5350020 \\ \mathrm{H} & 2.7129670 & -0.3507430 & 3.1095190 \\ \mathrm{H} & -4.1486850 & -2.0851690 & 1.7806350 \\ \mathrm{H} & -3.7593400 & -3.5231830 & 0.8199510 \\ \mathrm{H} & -4.9969280 & -2.3883400 & 0.2534730 \\ \mathrm{H} & -3.2734030 & -1.6528270 & -2.5660480 \\ \mathrm{H} & -1.5186270 & -1.7002610 & -2.2895480 \\ \mathrm{H} & -2.4996990 & -3.1419540 & -1.9910480 \\ \mathrm{C} & 0.0663920 & 1.3938400 & 2.8526730 \\ \mathrm{H} & -0.0811270 & 0.3605230 & 3.1793400 \\ \mathrm{H} & -0.8054680 & 1.9810150 & 3.1625610 \\ \mathrm{H} & 0.9365300 & 1.7981090 & 3.3821150 \\ \mathrm{H} & 0.3681810 & 2.5453090 & 1.0788450\end{array}$

Calculated exo product for entry 7/8:

Single point: SCF $(\omega \mathrm{B} 97 \mathrm{xd} / 6-311 \mathrm{G}(\mathrm{d}, \mathrm{p}))=-1370.6822$

Sum of electronic and thermal Free Energies $(\omega \mathrm{B} 97 \mathrm{xd} / 6-31 \mathrm{G}(\mathrm{d}))=-1369.9570$

$\operatorname{SCF}(\omega B 97 x d / 6-31 G(d))=-1370.38703$ 


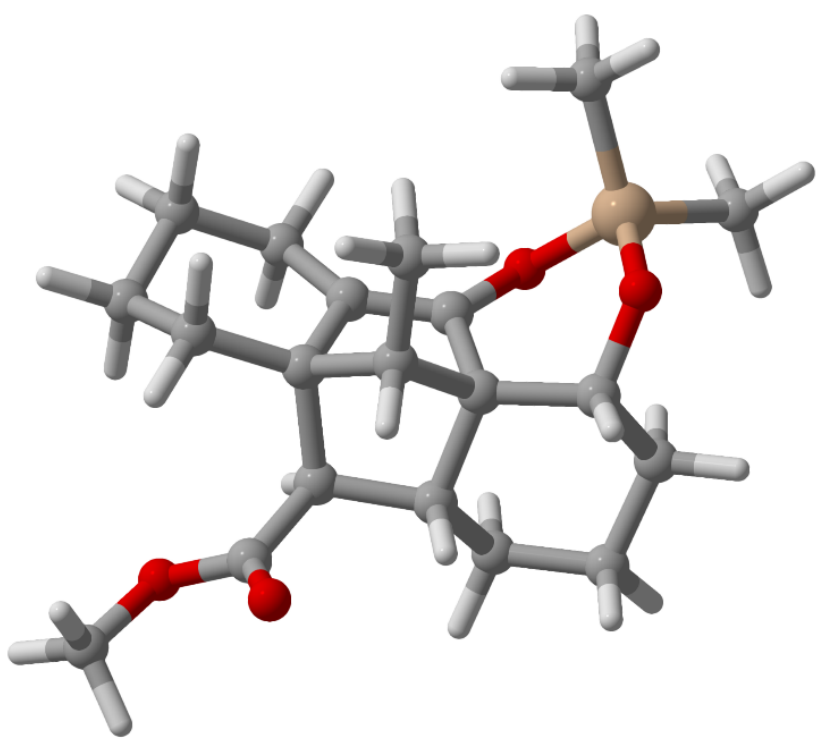

55

E 21 exo M0001

$\mathrm{O}^{-}{ }^{-} 2.8 \overline{3} 90820 \quad-0.5996910 \quad 1.1636190$

$\begin{array}{llll}\text { C } & 1.7420640 & -1.4758260 & 0.8836690\end{array}$

$\begin{array}{llll}\text { C } & 1.0076060 & -3.3345820 & -0.7687780\end{array}$

$\begin{array}{llll}\text { C } & -0.1056200 & -2.4317750 & -1.3115930\end{array}$

$\begin{array}{llll}\text { C } & 0.5137050 & -0.6692850 & 0.4648650\end{array}$

$\begin{array}{llll}\text { C } & 2.1547630 & -2.4953730 & -0.1993400\end{array}$

$\begin{array}{llll}\text { C } & 0.8512030 & 0.4678110 & -0.4945380\end{array}$

$\begin{array}{llll}\text { C } & -0.2006100 & 1.2809680 & -0.6041510\end{array}$

$\begin{array}{llll}\text { C } & -1.7656410 & -0.5935540 & -0.4074590\end{array}$

$\begin{array}{llll}\mathrm{C} & -0.3787240 & 0.0993440 & 1.4695810\end{array}$

$\begin{array}{llll}\mathrm{O} & 2.0693430 & 0.5586880 & -1.1046320\end{array}$

$\begin{array}{llll}\mathrm{C} & -1.2611370 & 0.7152100 & 0.3361900\end{array}$

C $\quad-0.4718900 \quad 2.4097840 \quad-1.5484750$

$\begin{array}{llll}\text { C } & -2.3213070 & 1.7348180 & 0.7396060\end{array}$

$\begin{array}{llll}\text { C } & -1.5136150 & 3.3845870 & -0.9838050\end{array}$

$\begin{array}{llll}\text { C } & -2.7307620 & 2.6350180 & -0.4306650\end{array}$

$\begin{array}{llll}\mathrm{Si} & 3.3608900 & 0.4571630 & -0.0139840\end{array}$

$\begin{array}{llll}\text { C } & 3.7026460 & 2.1041520 & 0.7872130\end{array}$

$\begin{array}{llll}\text { C } & 4.8461630 & -0.1329850 & -0.9749290\end{array}$

$\mathrm{H} \quad 1.5252330 \quad-2.0089140 \quad 1.8173760$

$\mathrm{H} \quad 1.3992010 \quad-3.9893770 \quad-1.5550910$

$\mathrm{H} \quad 0.5904700 \quad-3.9893030 \quad 0.0086540$

H $\quad 0.2767250 \quad-1.8017560 \quad-2.1267420$

$\mathrm{H} \quad-0.9248350 \quad-3.0346220 \quad-1.7214750$

$\mathrm{H} \quad 2.5920780 \quad-1.9437120 \quad-1.0384970$

H $\quad 2.9460780 \quad-3.1323610 \quad 0.2108450$

$\mathrm{H} \quad-0.8622020 \quad 1.9999490 \quad-2.4932270$

$\mathrm{H} \quad 0.4617900 \quad 2.9238460 \quad-1.8020770$ 


$\begin{array}{lrrr}\mathrm{H} & -3.1936350 & 1.2368540 & 1.1791450 \\ \mathrm{H} & -1.9040870 & 2.3730440 & 1.5294290 \\ \mathrm{H} & -1.8192710 & 4.0906330 & -1.7639970 \\ \mathrm{H} & -1.0640860 & 3.9767770 & -0.1748670 \\ \mathrm{H} & -3.1894660 & 2.0284120 & -1.2235400 \\ \mathrm{H} & -3.4926130 & 3.3486450 & -0.0969940 \\ \mathrm{H} & 2.7826350 & 2.5540100 & 1.1712110 \\ \mathrm{H} & 4.1520890 & 2.8014110 & 0.0718390 \\ \mathrm{H} & 4.3949960 & 1.9874960 & 1.6282650 \\ \mathrm{H} & 5.7240650 & -0.1865730 & -0.3214790 \\ \mathrm{H} & 4.6938540 & -1.1215410 & -1.4167190 \\ \mathrm{H} & 5.0757480 & 0.5691700 & -1.7844790 \\ \mathrm{C} & 0.2958160 & 1.0899210 & 2.4074920 \\ \mathrm{H} & 0.7444130 & 1.9237760 & 1.8603420 \\ \mathrm{H} & 1.0862360 & 0.5974270 & 2.9820320 \\ \mathrm{H} & -0.4296140 & 1.5022450 & 3.1168940 \\ \mathrm{H} & -0.9662730 & -0.6058070 & 2.0705920 \\ \mathrm{H} & -1.9131000 & -0.3772700 & -1.4687660 \\ \mathrm{C} & -3.0744760 & -1.0821110 & 0.1581920 \\ \mathrm{O} & -3.2182240 & -1.7077190 & 1.1852020 \\ \mathrm{O} & -4.1111860 & -0.6773990 & -0.5982850 \\ \mathrm{C} & -5.4022320 & -1.0032680 & -0.0879340 \\ \mathrm{H} & -5.5548470 & -0.5438390 & 0.8922340 \\ \mathrm{H} & -5.5169440 & -2.0857210 & 0.0079790 \\ \mathrm{H} & -6.1140500 & -0.6054860 & -0.8107840 \\ \mathrm{C} & -0.5991760 & -1.5729180 & -0.1609280 \\ \mathrm{H} & -0.9178230 & -2.2583350 & 0.6351580\end{array}$

Calculated endo transition state for entry 7/8:

Single point: $\mathrm{SCF}(\omega \mathrm{B} 97 \mathrm{xd} / 6-311 \mathrm{G}(\mathrm{d}, \mathrm{p}))=-1370.6267$

Sum of electronic and thermal Free Energies $(\omega \mathrm{B} 97 \mathrm{xd} / 6-31 \mathrm{G}(\mathrm{d}))=-1369.9039$ SCF $(\omega \mathrm{B} 97 \mathrm{xd} / 6-31 \mathrm{G}(\mathrm{d}))=-1370.32925$ 


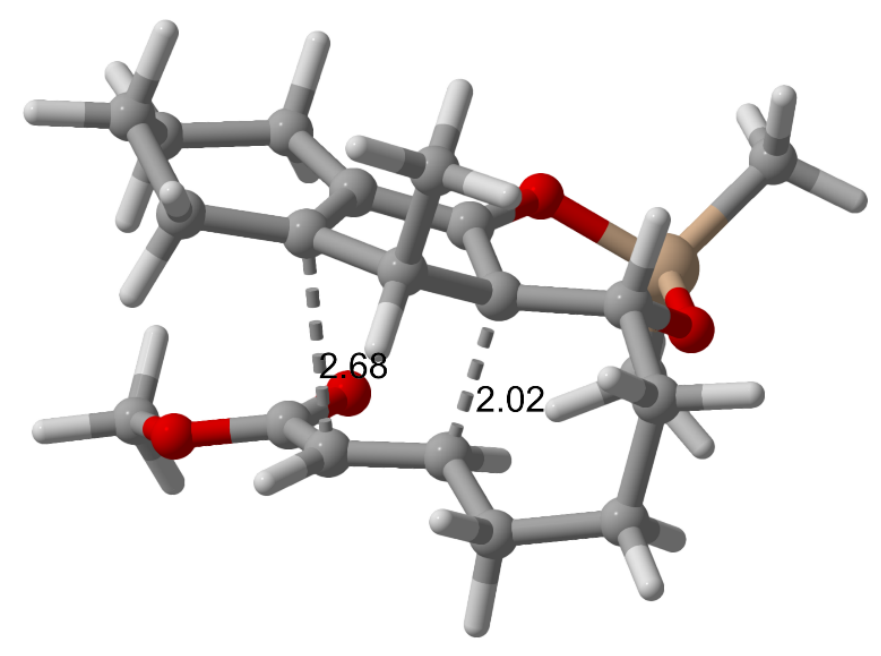

55

E_21_cis_TS_M0002

$\begin{array}{lrrr}\mathrm{O} & 2.9760570 & -0.0743980 & -0.0511210 \\ \mathrm{C} & 2.2896000 & 1.1389430 & -0.3647810 \\ \mathrm{C} & 2.5698010 & 1.9015570 & 2.0367450 \\ \mathrm{C} & 0.3699400 & 0.6154880 & 1.6773690 \\ \mathrm{C} & 1.0886100 & 1.7560880 & 2.3790050 \\ \mathrm{C} & 0.7886150 & 0.9141840 & -0.2758960 \\ \mathrm{C} & 2.8115700 & 2.2252120 & 0.5662460 \\ \mathrm{C} & 0.2708850 & -0.2182380 & -0.9488960 \\ \mathrm{C} & -1.1116320 & -0.0849300 & -1.1925400 \\ \mathrm{C} & -1.0223740 & 0.5374450 & 1.8008140 \\ \mathrm{C} & -0.2510180 & 2.0013360 & -0.5307660 \\ \mathrm{C} & -1.6506050 & -0.7444330 & 1.6749260 \\ \mathrm{O} & -1.0914150 & -1.7825390 & 1.3332190 \\ \mathrm{O} & -2.9831870 & -0.7206470 & 1.9650570 \\ \mathrm{C} & -3.6140190 & -1.9925890 & 1.9769410 \\ \mathrm{H} & 0.8927540 & -0.3348110 & 1.7575410 \\ \mathrm{O} & 0.9738650 & -1.3024480 & -1.2987270 \\ \mathrm{C} & -1.4820750 & 1.1632570 & -0.7620370 \\ \mathrm{C} & -2.0513780 & -1.1201960 & -1.7291430 \\ \mathrm{C} & -2.8857090 & 1.6771240 & -0.8654080 \\ \mathrm{C} & -3.5088970 & -0.6720580 & -1.5420550 \\ \mathrm{C} & -3.6946340 & 0.8181640 & -1.8482050 \\ \mathrm{Si} & 2.4974550 & -1.5652050 & -0.5996670 \\ \mathrm{C} & 3.6018990 & -2.1889490 & -1.9620520 \\ \mathrm{C} & 2.3134710 & -2.7378750 & 0.8299670 \\ \mathrm{H} & 2.5459900 & 1.4199590 & -1.3996700 \\ \mathrm{H} & 3.0958180 & 0.9727350 & 2.2851290 \\ \mathrm{H} & 3.0071720 & 2.6911580 & 2.6586080 \\ \mathrm{H} & 0.9914830 & 1.5728190 & 3.4567520 \\ \mathrm{H} & 0.5667310 & 2.7041120 & 2.1902290\end{array}$




$\begin{array}{lrrr}\mathrm{H} & 3.8835630 & 2.3495190 & 0.3772310 \\ \mathrm{H} & 2.3245990 & 3.1743960 & 0.3035890 \\ \mathrm{H} & -1.6143070 & 1.3823270 & 2.1354530 \\ \mathrm{H} & -4.6621670 & -1.8019170 & 2.2122070 \\ \mathrm{H} & -3.5293170 & -2.4913410 & 1.0071000 \\ \mathrm{H} & -3.1685820 & -2.6408600 & 2.7375830 \\ \mathrm{H} & -1.8690750 & -2.0735800 & -1.2225510 \\ \mathrm{H} & -1.8391620 & -1.2807220 & -2.7949320 \\ \mathrm{H} & -3.3576400 & 1.6428770 & 0.1248720 \\ \mathrm{H} & -2.8845190 & 2.7303990 & -1.1749410 \\ \mathrm{H} & -3.8078460 & -0.8441720 & -0.5020850 \\ \mathrm{H} & -4.1681250 & -1.2779440 & -2.1728060 \\ \mathrm{H} & -4.7547680 & 1.0881070 & -1.7920450 \\ \mathrm{H} & -3.3623300 & 1.0283480 & -2.8738770 \\ \mathrm{H} & 3.6871230 & -1.4503640 & -2.7654620 \\ \mathrm{H} & 3.2049690 & -3.1152520 & -2.3916140 \\ \mathrm{H} & 4.6083550 & -2.3972270 & -1.5830500 \\ \mathrm{H} & 3.1162130 & -2.6036930 & 1.5629050 \\ \mathrm{H} & 1.3491740 & -2.5578530 & 1.3190710 \\ \mathrm{H} & 2.3279460 & -3.7800240 & 0.4929380 \\ \mathrm{C} & 0.0663820 & 2.8417320 & -1.7814070 \\ \mathrm{H} & 0.2006350 & 2.2006100 & -2.6581740 \\ \mathrm{H} & 0.9807520 & 3.4270560 & -1.6363010 \\ \mathrm{H} & -0.7462330 & 3.5455630 & -1.9911960 \\ \mathrm{H} & -0.3767270 & 2.6853170 & 0.3124390\end{array}$

Calculated exo transition state for entry 7/8:

Single point: SCF $(\omega \mathrm{B} 97 \mathrm{xd} / 6-311 \mathrm{G}(\mathrm{d}, \mathrm{p}))=-1370.6157$

Sum of electronic and thermal Free Energies $(\omega \mathrm{B} 97 \mathrm{xd} / 6-31 \mathrm{G}(\mathrm{d}))=-1369.8934$

$\operatorname{SCF}(\omega \mathrm{B} 97 \mathrm{xd} / 6-31 \mathrm{G}(\mathrm{d}))=-1370.31781$

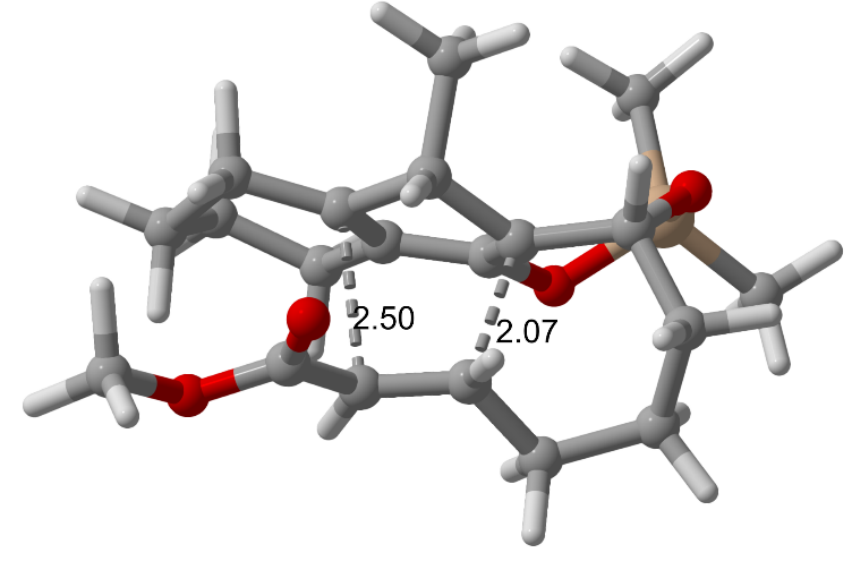

55

E_21_exo_TS_M0002

$\mathrm{O}-2.9952470 \quad-0.5947590 \quad 1.0686640$ 


\begin{tabular}{|c|c|c|c|}
\hline $\mathrm{C}$ & 1.7614010 & -1.3247090 & 1.1509540 \\
\hline $\mathrm{C}$ & 1.8065010 & -2.6047970 & -1.0756880 \\
\hline $\mathrm{C}$ & 0.3983890 & -2.2016490 & -1.5811050 \\
\hline $\mathrm{C}$ & 0.5907610 & -0.5012920 & 0.6321290 \\
\hline $\mathrm{C}$ & 1.9324670 & -2.6774880 & 0.4475320 \\
\hline $\mathrm{C}$ & 0.8186760 & 0.5503980 & -0.2750210 \\
\hline $\mathrm{C}$ & -0.3095260 & 1.3771680 & -0.3837540 \\
\hline $\mathrm{C}$ & -1.7473700 & -1.0987120 & -0.8255650 \\
\hline $\mathrm{C}$ & -0.5664980 & -0.0189320 & 1.4957820 \\
\hline $\mathrm{O}$ & 1.9486900 & 0.6957200 & -1.0103870 \\
\hline $\mathrm{C}$ & -1.2365370 & 0.9332280 & 0.5400290 \\
\hline $\mathrm{C}$ & -0.5749810 & 2.4542840 & -1.3909810 \\
\hline $\mathrm{C}$ & -2.5235510 & 1.6545740 & 0.7916170 \\
\hline $\mathrm{C}$ & -1.7632080 & 3.3218290 & -0.9513330 \\
\hline $\mathrm{C}$ & -2.9314780 & 2.4680180 & -0.4414850 \\
\hline $\mathrm{Si}$ & 3.3535410 & 0.5499950 & -0.0682090 \\
\hline $\mathrm{C}$ & 3.6778790 & 2.1812050 & 0.7734430 \\
\hline $\mathrm{C}$ & 4.7458910 & 0.054 & -1.1992240 \\
\hline $\mathrm{H}$ & 1.6095760 & -1.5158510 & 2.2196350 \\
\hline $\mathrm{H}$ & 2.5443980 & -1.89 & -1.4603740 \\
\hline $\mathrm{H}$ & 2.0842720 & -3.5773960 & -1.4950550 \\
\hline $\mathrm{H}$ & 0.4813640 & -1.3927540 & -2.3156380 \\
\hline $\mathrm{H}$ & -0.0542390 & -3.048 & -2.1124640 \\
\hline $\mathrm{H}$ & 2.9196640 & -3.0610750 & 0.7254490 \\
\hline $\mathrm{H}$ & 1.1983850 & -3.38 & 0.8515320 \\
\hline $\mathrm{H}$ & -0.7950890 & 1.9895260 & -2.3629540 \\
\hline $\mathrm{H}$ & 0.3227750 & 3.0641260 & -1.5424220 \\
\hline $\mathrm{H}$ & -3.3124710 & 0.9549640 & 1.0867610 \\
\hline $\mathrm{H}$ & -2.3812610 & & 1.6507680 \\
\hline $\mathrm{H}$ & -2.0893140 & 3.9537140 & -1.7844450 \\
\hline $\mathrm{H}$ & -1.4391790 & 3.9973920 & -0.1479030 \\
\hline $\mathrm{H}$ & -3.2581640 & 1.7742330 & -1.2259470 \\
\hline $\mathrm{H}$ & -3.7879320 & 3.1064510 & -0.1988660 \\
\hline $\mathrm{H}$ & 2.7783410 & 2.5188550 & 1.2994900 \\
\hline $\mathrm{H}$ & 3.9589760 & 2.9533210 & 0.0489980 \\
\hline $\mathrm{H}$ & 4.4838560 & 2.0955100 & 1.5100250 \\
\hline $\mathrm{H}$ & 5.6897050 & 0.0039120 & -0.6453640 \\
\hline $\mathrm{H}$ & & -0.9229120 & -1.6597370 \\
\hline $\mathrm{H}$ & 4.8667270 & 0.7919650 & -2.0001930 \\
\hline $\mathrm{C}$ & -0.0832210 & 0.7272270 & 2.7519560 \\
\hline $\mathrm{H}$ & 0.5707870 & 1.5639270 & 2.4863070 \\
\hline $\mathrm{H}$ & 0.4694810 & 0.0547310 & 3.4159280 \\
\hline $\mathrm{H}$ & -0.9372370 & 1.1183020 & 3.3150380 \\
\hline $\mathrm{H}$ & -1.2393550 & -0.8222660 & 1.8079320 \\
\hline $\mathrm{H}$ & -1.8391240 & -0.5787310 & -1.7734910 \\
\hline $\mathrm{C}$ & -2.9777870 & -1.3465190 & -0.1011080 \\
\hline
\end{tabular}




$\begin{array}{lllr}\mathrm{O} & -3.1003820 & -1.9382440 & 0.9604360 \\ \mathrm{O} & -4.0483850 & -0.8050080 & -0.7411200 \\ \mathrm{C} & -5.2990620 & -0.9971930 & -0.0951610 \\ \mathrm{H} & -5.3032080 & -0.5420830 & 0.8998290 \\ \mathrm{H} & -5.5255770 & -2.0616780 & 0.0097960 \\ \mathrm{H} & -6.0389930 & -0.5132270 & -0.7336000 \\ \mathrm{C} & -0.5777150 & -1.7856960 & -0.5026610 \\ \mathrm{H} & -0.7078660 & -2.5066680 & 0.3030120\end{array}$

\section{Computation References:}

1 Gaussian 16, Revision A.03, M. J. Frisch, G. W. Trucks, H. B. Schlegel, G. E. Scuseria, M. A. Robb, J. R. Cheeseman, G. Scalmani, V. Barone, G. A. Petersson, H. Nakatsuji, X. Li, M. Caricato, A. V. Marenich, J. Bloino, B. G. Janesko, R. Gomperts, B. Mennucci, H. P. Hratchian, J. V. Ortiz, A. F. Izmaylov, J. L. Sonnenberg, D. Williams-Young, F. Ding, F. Lipparini, F. Egidi, J. Goings, B. Peng, A. Petrone, T. Henderson, D. Ranasinghe, V. G. Zakrzewski, J. Gao, N. Rega, G. Zheng, W. Liang, M. Hada, M. Ehara, K. Toyota, R. Fukuda, J. Hasegawa, M. Ishida, T. Nakajima, Y. Honda, O. Kitao, H. Nakai, T. Vreven, K. Throssell, J. A. Montgomery, Jr., J. E. Peralta, F. Ogliaro, M. J. Bearpark, J. J. Heyd, E. N. Brothers, K. N. Kudin, V. N. Staroverov, T. A. Keith, R. Kobayashi, J. Normand, K. Raghavachari, A. P. Rendell, J. C. Burant, S. S. Iyengar, J. Tomasi, M. Cossi, J. M. Millam, M. Klene, C. Adamo, R. Cammi, J. W. Ochterski, R. L. Martin, K. Morokuma, O. Farkas, J. B. Foresman, and D. J. Fox, Gaussian, Inc., Wallingford CT, 2016.

2 (a) Spartan 18, Wavefunction Inc., Irvine CA. (b) Shao, Y.; Gan, Z.; Epifanovsky, E.; Gilbert, A. T. B.; Wormit, M.; Kussmann, J.; Lange, A. W.; Behn, A.; Deng, J.; Feng, X.; Ghosh, D.; Goldey, M.; Horn, P. R.; Jacobson, L. D.; Kaliman, I.; Khaliullin, R. Z.; Kuś, T.; Landau, A.; Liu, J.; Proynov, E. I.; Rhee, Y. M.; Richard, R. M.; Rohrdanz, M. A.; Steele, R. P.; Sundstrom, E. J.; Woodcock, H. L., III; Zimmerman, P. M.; Zuev, D.; Albrecht, B.; Alguire, E.; Austin, B.; Beran, G. J. O.; Bernard, Y. A.; Berquist, E.; Brandhorst, K.; Bravaya, K. B.; Brown, S. T.; Casanova, D.; Chang, C.-M.; Chen, Y.; Chien, S. H.; Closser, K. D.; Crittenden, D. L.; Diedenhofen, M.; DiStasio, R. A., Jr.; Do, H.; Dutoi, A. D.; Edgar, R. G.; Fatehi, S.; FustiMolnar, L.; Ghysels, A.; Golubeva-Zadorozhnaya, A.; Gomes, J.; Hanson-Heine, M. W. D.; Harbach, P. H. P.; Hauser, A. W.; Hohenstein, E. G.; Holden, Z. C.; Jagau, T.-C.; Ji, H.; Kaduk, B.; Khistyaev, K.; Kim, J.; Kim, J.; King, R. A.; Klunzinger, P.; Kosenkov, D.; Kowalczyk, T.; Krauter, C. M.; Lao, K. U.; Laurent, A. D.; Lawler, K. V.; Levchenko, S. V.; Lin, C. Y.; Liu, F.; Livshits, E.; Lochan, R. C.; Luenser, A.; Manohar, P.; Manzer, S. F.; Mao, S.-P.; Mardirossian, N.; Marenich, A. V.; Maurer, S. A.; Mayhall, N. J.; Neuscamman, E.; Oana, C. M.; OlivaresAmaya, R.; O’Neill, D. P.; Parkhill, J. A.; Perrine, T. M.; Peverati, R.; Prociuk, A.; Rehn, D. R.; Rosta, E.; Russ, N. J.; Sharada, S. M.; Sharma, S.; Small, D. W.; Sodt, A.; Stein, T.; Stück, D.; Su, Y.-C.; Thom, A. J. W.; Tsuchimochi, T.; Vanovschi, V.; Vogt, L.; Vydrov, O.; Wang, T.; Watson, M. A.; Wenzel, J.; White, A.; Williams, C. F.; Yang, J.; Yeganeh, S.; Yost, S. R.; You, Z.-Q.; Zhang, I. Y.; Zhang, X.; Zhao, Y.; Brooks, B. R.; Chan, G. K. L.; Chipman, D. M.;

Cramer, C. J.; Goddard Iii, W. A.; Gordon, M. S.; Hehre, W. J.; Klamt, A.; Schaefer, H. F., III; Schmidt, M. W.; Sherrill, C. D.; Truhlar, D. G.; Warshel, A.; Xu, X.; Aspuru-Guzik, A.; Baer, R.; Bell, A. T.; Besley, N. A.; Chai, J.-D.; Dreuw, A.; Dunietz, B. D.; Furlani, T. R.; Gwaltney, 
S. R.; Hsu, C.-P.; Jung, Y.; Kong, J.; Lambrecht, D. S.; Liang, W.; Ochsenfeld, C.; Rassolov, V. A.; Slipchenko, L. V.; Subotnik, J. E.; Van Voorhis, T.; Herbert, J. M.; Krylov, A. I.; Gill, P. M. W.; Head-Gordon, M. Advances in Molecular Quantum Chemistry Contained in the Q-Chem 4 Program Package. Molecular Physics 2014, 113, 184-215.

${ }^{3}$ CYLview20; Legault, C. Y., Université de Sherbrooke, 2020 (http://www.cylview.org). 